\title{
AFIP-4 Irradiation Summary Report
}

D. M. Perez

M. A. Lillo

G. S. Chang

G. A. Roth

N. E. Woolstenhulme

D. M. Wachs

January 2012

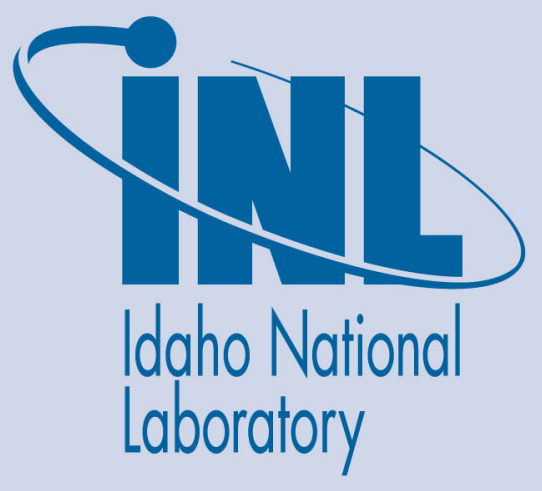

The INL is a U.S. Department of Energy National Laboratory operated by Battelle Energy Alliance 
INL/EXT-11-23297

Rev. 1

\title{
AFIP-4 Irradiation Summary Report
}

\author{
D. M. Perez \\ M. A. Lillo \\ G. S. Chang \\ G. A. Roth \\ N. E. Woolstenhulme \\ D. M. Wachs \\ January 2012

\section{Idaho National Laboratory \\ Idaho Falls, Idaho 83415} \\ http://www.inl.gov \\ Prepared for the \\ U.S. Department of Energy \\ Office of National Nuclear Security Administration \\ Under DOE Idaho Operations Office \\ Contract DE-AC07-05ID14517
}




\section{DISCLAIMER}

This information was prepared as an account of work sponsored by an agency of the U.S. Government. Neither the U.S. Government nor any agency thereof, nor any of their employees, makes any warranty, expressed or implied, or assumes any legal liability or responsibility for the accuracy, completeness, or usefulness, of any information, apparatus, product, or process disclosed, or represents that its use would not infringe privately owned rights. References herein to any specific commercial product, process, or service by trade name, trade mark, manufacturer, or otherwise, does not necessarily constitute or imply its endorsement, recommendation, or favoring by the U.S. Government or any agency thereof. The views and opinions of authors expressed herein do not necessarily state or reflect those of the U.S. Government or any agency thereof. 
AFIP-4 Irradiation Summary Report

INL/EXT-11-23297

January 2012

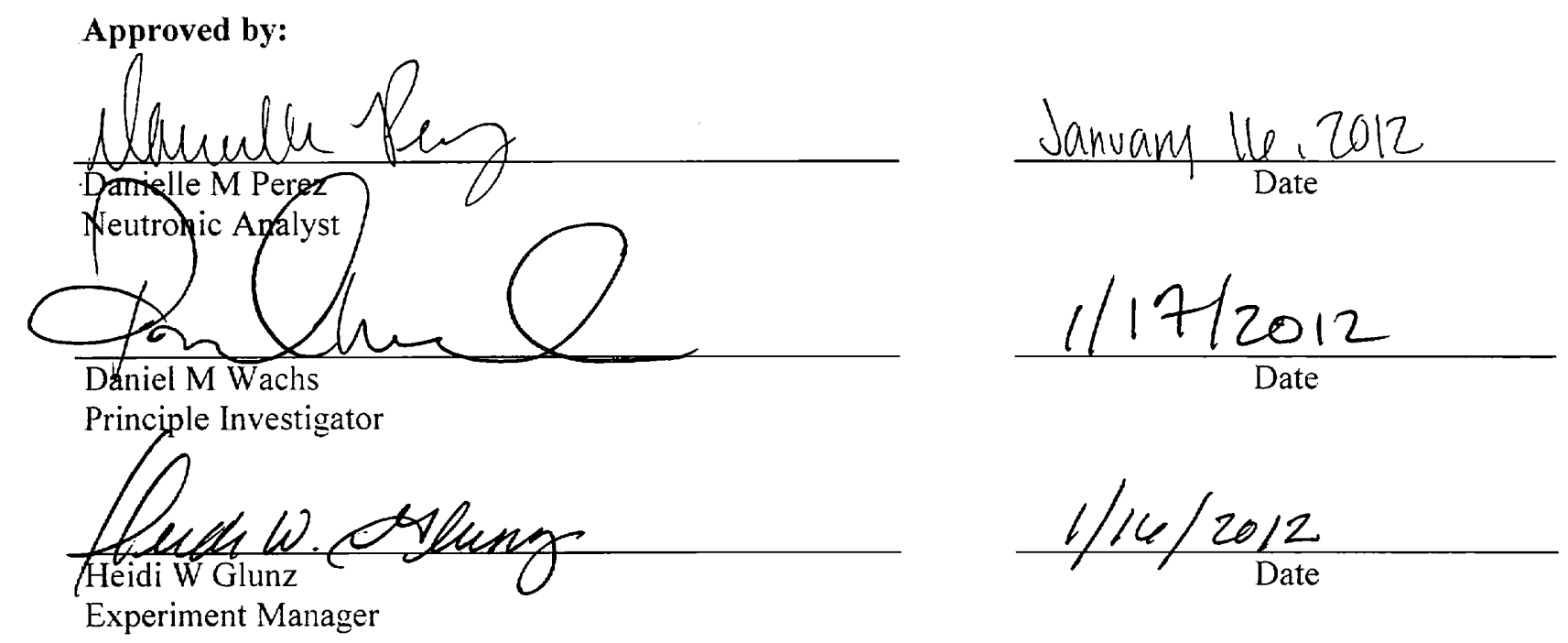




\section{SUMMARY}

The Advanced Test Reactor (ATR) Full size plate In center flux trap Position (AFIP) experiment AFIP-4 was designed to evaluate the performance of monolithic uranium-molybdenum (U-Mo) fuels at a scale prototypic of research reactor fuel plates. The AFIP-4 test further examine the fuel/clad interface and its behavior under extreme conditions. After irradiation, fission gas retention measurements will be performed during post irradiation (PIE) $)^{1,2}$.

The following report summarizes the life of the AFIP-4 experiment through end of irradiation, including a brief description of the safety analysis, as-run neutronic analysis results, hydraulic testing results, and thermal analysis results. 


\section{CONTENTS}

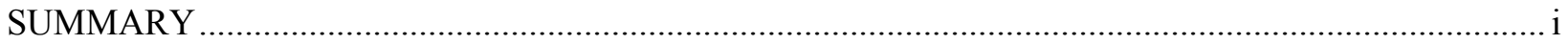

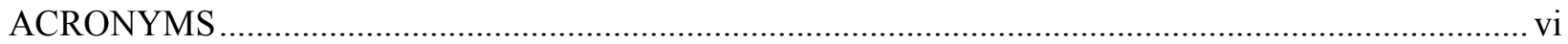

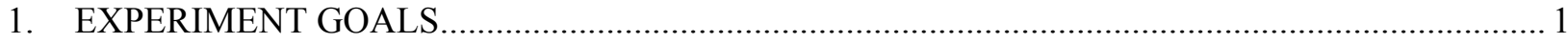

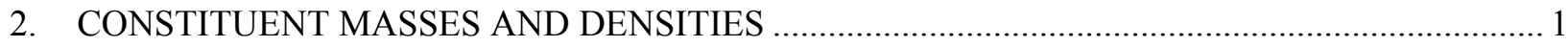

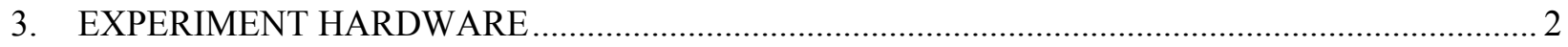

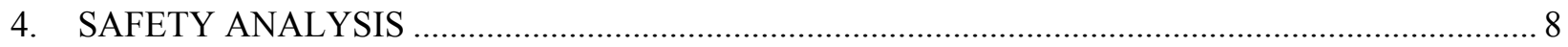

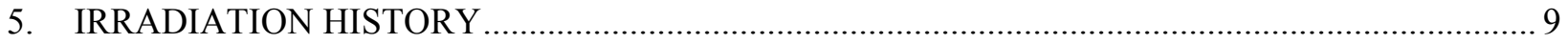

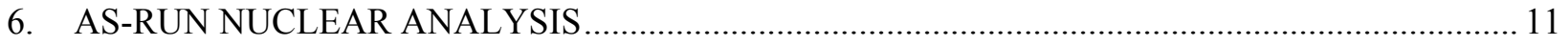

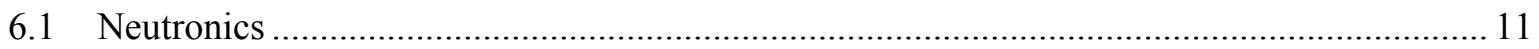

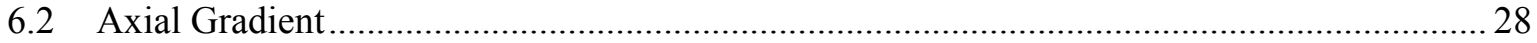

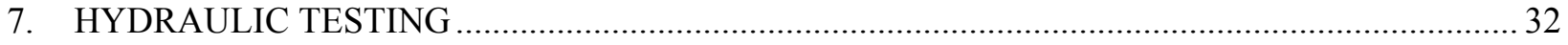

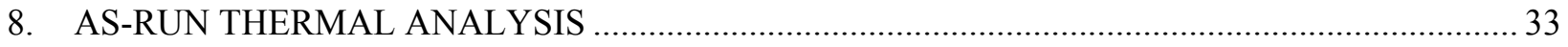

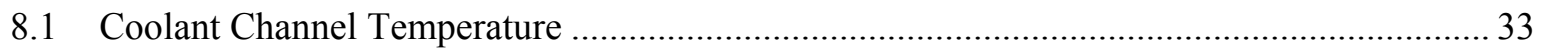

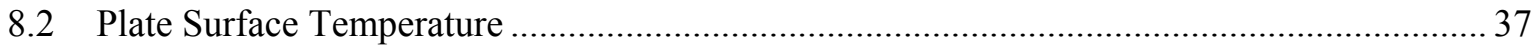

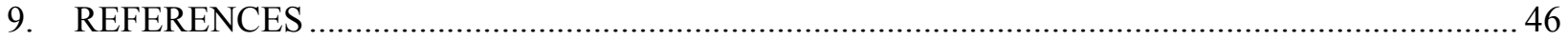

Appendix A: Individual Plate Power and Fission Density Plots............................................................. 47

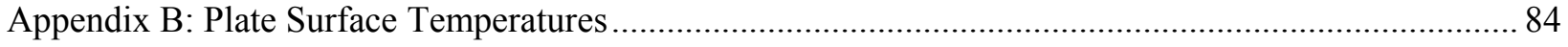




\section{FIGURES}

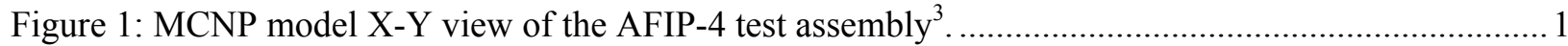

Figure 2: DWG 635793 ATR Full Size Plate in Center Flux Trap Position (AFIP) Test Train

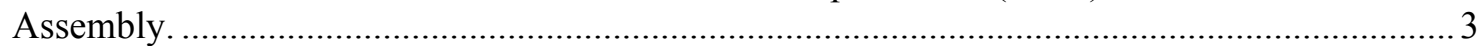

Figure 3: Isometric view of the AFIP-4 Test Train Assembly.............................................................. 4

Figure 4: DWG-759268 REV 0 AFIP-4 Frame Assembly. ...................................................................... 5

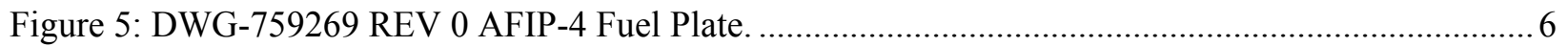

Figure 6: Radial Cross Section of the AFIP Test Train Assembly. .......................................................... 7

Figure 7: Hourly constrained lobe power history for Cycle 144B ......................................................... 9

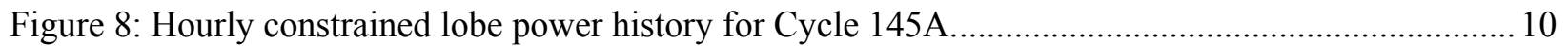

Figure 9: AFIP-4 Axial Neutron Flux Profile for Plates in Position A. ${ }^{3}$..................................................28

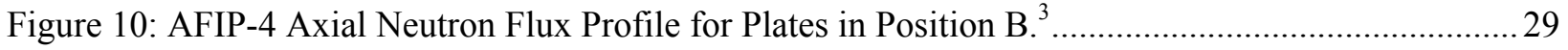

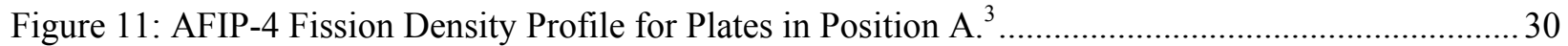

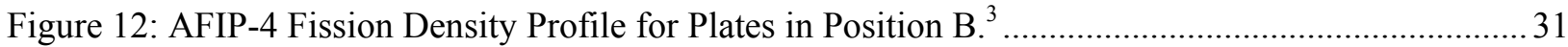

Figure 13: Coolant channel temperatures as a function of location along the AFIP-4 test assembly

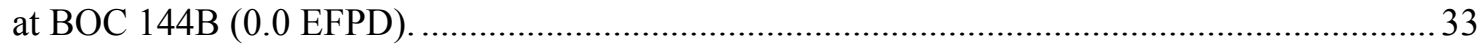

Figure 14: Coolant channel temperatures as a function of location along the AFIP-4 test assembly

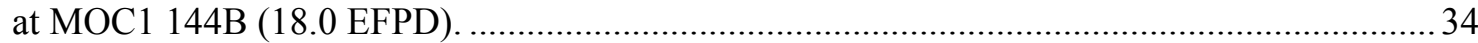

Figure 15: Coolant channel temperatures as a function of location along the AFIP-4 test assembly at MOC2 144B (38.0 EFPD).

Figure 16: Coolant channel temperatures as a function of location along the AFIP-4 test assembly at EOC 144B (51.7 EFPD).

Figure 17: Coolant channel temperatures as a function of location along the AFIP-4 test assembly at BOC 145A (0 EFPD, cumulative 51.7 EFPD).

Figure 18: Coolant channel temperatures as a function of location along the AFIP-4 test assembly at MOC1 145A (18 EFPD, cumulative 69.7 EFPD).

Figure 19: Coolant channel temperatures as a function of location along the AFIP-4 test assembly at MOC2 145A (38 EFPD, cumulative 89.7 EFPD).

Figure 20: Coolant channel temperatures as a function of location along the AFIP-4 test assembly at EOC 145A (54.7 EFPD, cumulative 106.4 EFPD). 


\section{TABLES}

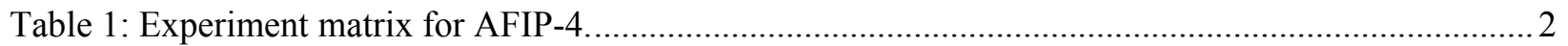

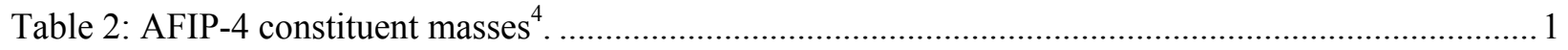

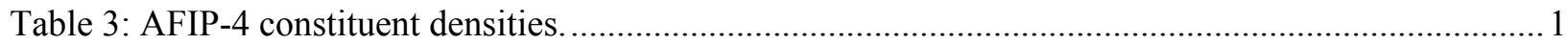

Table 4. AFIP-4 Irradiation Hardware Drawing List............................................................................. 2

Table 5: Summary table of the safety analyses done for the AFIP-4 experiment..................................... 8

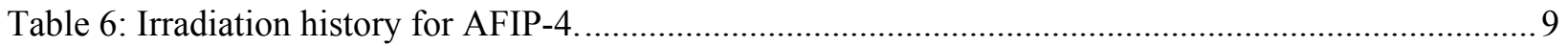

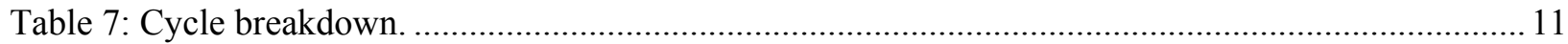

Table 8: Cycle 144B, MCNP-Calculated HGRs and Neutron Flux for AFIP-4 Fuel Foils in

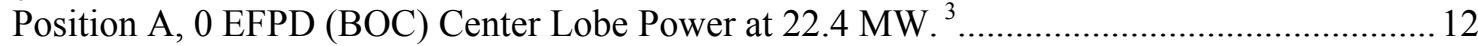

Table 9: Cycle 144B, MCNP-Calculated HGRs and Neutron Flux for AFIP-4 Fuel Foils in Position B, 0 EFPD (BOC), Center Lobe Power at 22.4 MW. ${ }^{3}$ 13

Table 10: Cycle 144B, MCNP-Calculated HGR, Neutron Flux, Depletion, and Fission Density for AFIP-4 Fuel Foils in Position A, 18.0 EFPD (MOC1), Center Lobe Power at 22.4 MW (18EFPD Cumulative). ${ }^{3}$

Table 11: Cycle 144B, MCNP-Calculated HGR, Neutron Flux, Depletion, and Fission Density for AFIP-4 Fuel Foils in Position B, 18 EFPD (MOC1), Center Lobe Power at 22.4 MW (18 EFPD Cumulative). ${ }^{3}$

Table 12: Cycle 144B, MCNP-Calculated HGR, Neutron Flux, Depletion, and Fission Density for AFIP-4 Fuel Foils in Position A, 38 EFPD (MOC2), Center Lobe Power at 22.4 MW (38 EFPD Cumulative). ${ }^{3}$

Table 13: Cycle 144B, MCNP-Calculated HGR, Neutron Flux, Depletion, and Fission Density for AFIP-4 Fuel Foils in Position B, 38 EFPD (MOC2), Center Lobe Power at 22.4 MW (38 EFPD Cumulative). ${ }^{3}$

Table 14: Cycle 144B, MCNP-Calculated HGR, Neutron Flux, Depletion, and Fission Density for AFIP-4 Fuel Foils in Position A, 51.7 EFPD (EOC), Center Lobe Power at 22.4MW (51.7 EFPD Cumulative). ${ }^{3}$

Table 15: Cycle 144B, MCNP-Calculated HGR, Neutron Flux, Depletion, and Fission Density for AFIP-4 Fuel Foils in Position B, 51.7 EFPD (EOC), Center Lobe Power at 22.4 MW (51.7 EFPD Cumulative). ${ }^{3}$....

Table 16: Cycle 145A, MCNP-Calculated HGR, Neutron Flux, Depletion, and Fission Density for AFIP-4 Fuel Foils in Position A, 0 EFPD (BOC), Center Lobe Power at 23.2 MW (51.7 EFPD Cumulative). ${ }^{3}$.

Table 17: Cycle 145A, MCNP-Calculated HGR, Neutron Flux, Depletion, and Fission Density for AFIP-4 Fuel Foils in Position B, 0 EFPD (BOC), Center Lobe Power at 23.2 MW (51.7 EFPD Cumulative). ${ }^{3}$

Table 18: Cycle 145A, MCNP-Calculated HGR, Neutron Flux, Depletion, and Fission Density for AFIP-4 Fuel Foils in Position A, 18.0 EFPD (MOC1), Center Lobe Power at 23.2 MW (69.7 EFPD Cumulative). ${ }^{3}$...... 
Table 19: Cycle 145A, MCNP-Calculated HGR, Neutron Flux, Depletion, and Fission Density for AFIP-4 Fuel Foils in Position B, 18.0 EFPD (MOC1), Center Lobe Power at 23.2 MW (69.7 EFPD Cumulative). ${ }^{3}$.

Table 20: Cycle 145A, MCNP-Calculated HGR, Neutron Flux, Depletion, and Fission Density for AFIP-4 Fuel Foils in Position A, 38.0 EFPD (MOC2), Center Lobe Power at 23.2 MW (89.7 EFPD Cumulative). ${ }^{3}$.

Table 21: Cycle 145A, MCNP-Calculated HGR, Neutron Flux, Depletion, and Fission Density for AFIP-4 Fuel Foils in Position B, 38.0 EFPD (MOC2), Center Lobe Power at 23.2 MW (89.7 EFPD Cumulative). ${ }^{3}$.

Table 22: Cycle 145A, MCNP-Calculated HGR, Neutron Flux, Depletion, and Fission Density for AFIP-4 Fuel Foils in Position A, 54.7 EFPD (EOC), Center Lobe Power at 23.2 MW (106.4 EFPD Cumulative). ${ }^{3}$.

Table 23: Cycle 145A, MCNP-Calculated HGR, Neutron Flux, Depletion, and Fission Density for AFIP-4 Fuel Foils in Position B, 54.7 EFPD (EOC), Center Lobe Power at 23.2 MW (106.4 EFPD Cumulative). ${ }^{3}$.. 27

Table 24: AFIP irradiation vehicle flow conditions for each orifice configuration ${ }^{5}$.................................... 32

Table 25: Temperature $\left({ }^{\circ} \mathrm{C}\right)$ map of the east side of position A at EOC 144B (51.7 EFPD).....................38

Table 26: Temperature $\left({ }^{\circ} \mathrm{C}\right)$ map of the west side of position A at EOC 144B (51.7 EFPD).................. 39

Table 27: Temperature $\left({ }^{\circ} \mathrm{C}\right)$ map of the east side of position B at EOC 144B (51.7 EFPD) .................... 40

Table 28: Temperature $\left({ }^{\circ} \mathrm{C}\right)$ map of the west side of position B at EOC 144B (51.7 EFPD)................... 41

Table 29: Temperature $\left({ }^{\circ} \mathrm{C}\right)$ map of the east side of position A at EOC 145A (106.4 cumulative).......... 42

Table 30: Temperature $\left({ }^{\circ} \mathrm{C}\right)$ map of the west side of position A at EOC 145A (106.4 cumulative) .......... 43

Table 31: Temperature $\left({ }^{\circ} \mathrm{C}\right)$ map of the east side of position B at EOC 145A (106.4 cumulative)........... 44

Table 32: Temperature $\left({ }^{\circ} \mathrm{C}\right)$ map of the west side of position B at EOC 145A (106.4 cumulative)......... 45 


\section{ACRONYMS}

$\mathrm{Al}$

ATR

AFIP

CFT

DAS

DNBR

EFPD

GTRI

FB

FD

FIR

HIP

MCNP

Mo

RERTR

U

U-Mo

$\mathrm{Zr}$
Aluminum

Advanced Test Reactor

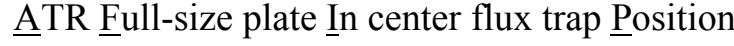

Center Flux Trap

Data Acquisition System

Departure from Nucleate Boiling Ratio

Effective Full Power Days

Global Threat Reduction Initiative

Friction Bond

Fuel Development

Flow Instability Ratio

Hot Isostatic Pressing

Monte Carlo N-Particle

Molybdenum

Reduced Enrichment Research and Test Reactor

Uranium

Uranium-Molybdenum

Zirconium 


\section{AFIP-4 Irradiation Summary Report \\ 1. EXPERIMENT GOALS}

In support of the Global Threat Reduction Initiative (GTRI) Fuel Development (FD) program (historically known as Reduced Enrichment for Research and Test Reactors (RERTR)), the Advanced Test Reactor (ATR) Full size plate In center flux trap Position (AFIP) experiment AFIP-4 was designed to evaluate the performance of monolithic uranium-molybdenum (U-Mo) fuels at a scale prototypic of research reactor fuel plates. The AFIP-4 test further examines the fuel-to-cladding interface and its behavior under extreme conditions. After irradiation, fission gas retention measurements will be performed during post irradiation (PIE) ${ }^{1,2}$.

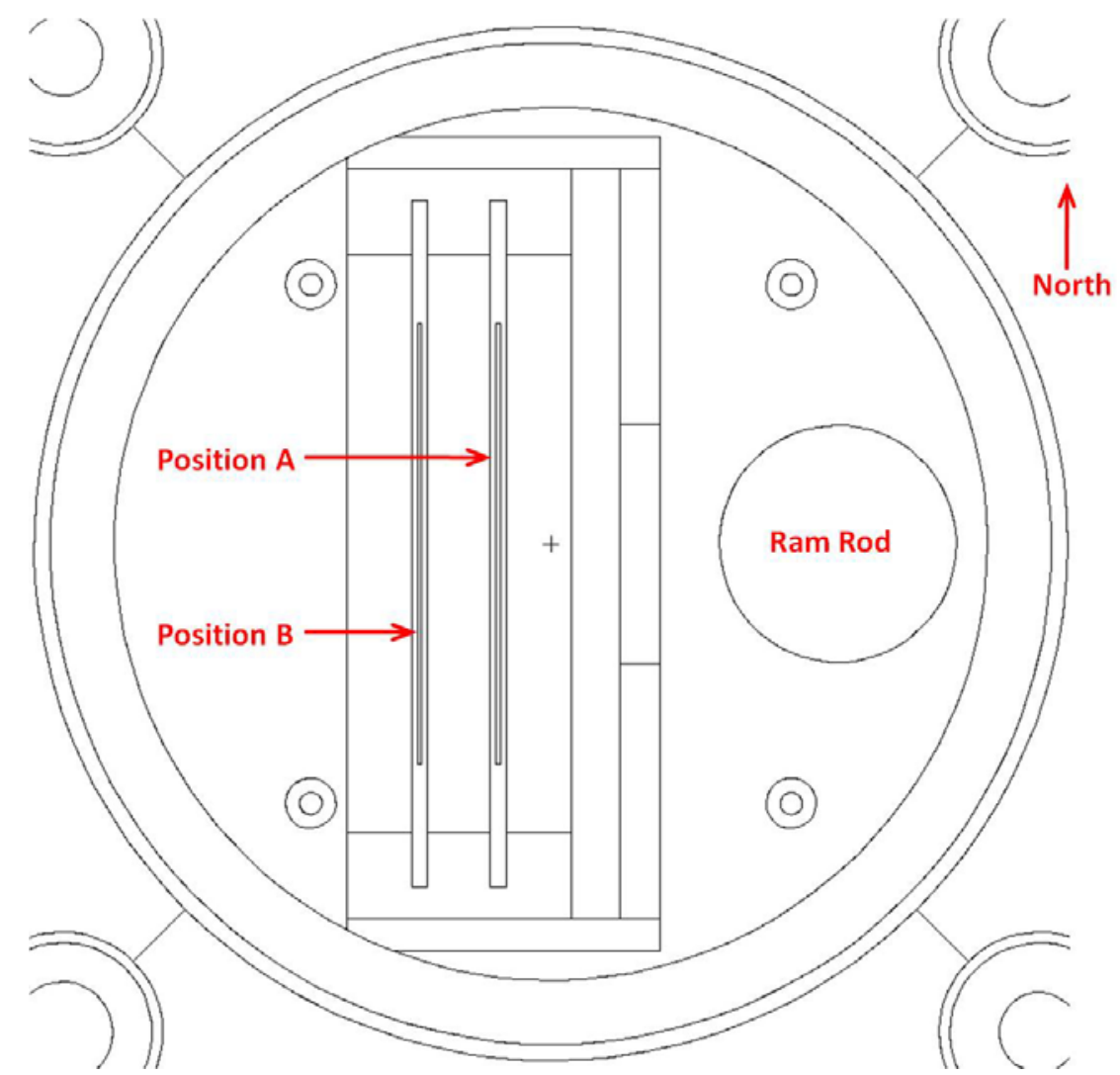

Figure 1: MCNP model X-Y view of the AFIP-4 test assembly ${ }^{3}$.

The AFIP-4 experiment was irradiated in the ATR Center Flux Trap (CFT) position. The test assembly holds 2 full length frame assemblies, designated as A and B, a ramrod and four flux monitor wires. Frame $\mathrm{A}$ is located closest to the ramrod and frame B is on the outer (west) location in the assembly, see Figure 1. Each frame assembly holds six plates for a total of 12 plates. Each plate is approximately 7.5 inches in length, 2.21 inches in width and 0.050 inches in thickness. The fuel zone was approximately 6.25 inches in length, 1.40 inches in width and 0.013 inches in thickness. A diffusion barrier of $\sim 0.001$ inch thick zirconium was bonded to the top and bottom surface of the fuel foil. The plates located in position A were friction bonded (FB) plates and the plates in position B were hot isostatic pressed (HIP). The Experiment matrix is shown in Table 1. 
Table 1: Experiment matrix for AFIP-4.

\begin{tabular}{|c|c|c|}
\hline \multicolumn{3}{|c|}{ RERTR AFIP-4 Experiment Matrix } \\
\hline $\begin{array}{c}\text { Assembly } \\
\text { Position }\end{array}$ & A Position & B Position \\
\hline \multirow{6}{*}{ Top } & A1 & B1 \\
\hline & $\begin{array}{c}\text { U-10 Mo } \\
\text { Zr Co-Roll } \\
\text { FB } \\
\text { L1B31Z }\end{array}$ & $\begin{array}{c}\text { U-10Mo } \\
\text { Zr Co-Roll } \\
\text { HIP } \\
\text { L1H34Z }\end{array}$ \\
\hline & $\mathbf{A 2}$ & B2 \\
\hline & $\begin{array}{c}\text { U-10Mo } \\
\text { Zr Co-Roll } \\
\text { FB } \\
\text { L1B33Z }\end{array}$ & $\begin{array}{c}\text { U-10Mo } \\
\text { Zr Co-Roll } \\
\text { HIP } \\
\text { L1H35Z }\end{array}$ \\
\hline & A3 & B3 \\
\hline & $\begin{array}{c}\text { U-10Mo } \\
\text { Zr Co-Roll } \\
\text { FB } \\
\text { L1B32Z }\end{array}$ & $\begin{array}{c}\text { U-10Mo } \\
\text { Zr Co-Roll } \\
\text { HIP } \\
\text { L1H36Z }\end{array}$ \\
\hline \multirow{10}{*}{ Bottom } & A4 & B4 \\
\hline & $\begin{array}{c}\text { U-10 Mo } \\
\text { Zr Co-Roll } \\
\text { FB } \\
\text { L1B51Z }\end{array}$ & $\begin{array}{c}\text { U-10Mo } \\
\text { Zr Co-Roll } \\
\text { HIP } \\
\text { L1H37Z }\end{array}$ \\
\hline & A5 & B5 \\
\hline & U-10Mo & U-10Mo \\
\hline & $\begin{array}{c}\mathrm{Zr} \mathrm{Co}-\mathrm{Roll} \\
\text { FB }\end{array}$ & $\begin{array}{c}\text { Zr Co-Roll } \\
\text { HIP }\end{array}$ \\
\hline & L1B52Z & L1H38Z \\
\hline & A6 & B6 \\
\hline & U-10Mo & U-10Mo \\
\hline & $\begin{array}{c}\mathrm{Zr} \mathrm{Co}-\mathrm{Roll} \\
\text { FB }\end{array}$ & $\begin{array}{c}\text { Zr Co-Roll } \\
\text { HIP }\end{array}$ \\
\hline & L1B53Z & L1H39Z \\
\hline
\end{tabular}




\section{CONSTITUENT MASSES AND DENSITIES}

The constituent masses and densities for the 12 plates were obtained from the as-built data package plate $^{4}$ summary sheets. Table 2 summarizes the constituent masses for the plates and Table 3 summarizes the constituent densities for the plates.

Table 2: AFIP-4 constituent masses ${ }^{4}$.

\begin{tabular}{|c|c|c|c|c|c|}
\hline $\begin{array}{c}\text { Fuel Plate } \\
\text { Location }\end{array}$ & $\begin{array}{c}\text { Fuel Plate } \\
\text { ID }\end{array}$ & $\begin{array}{c}\text { Total-U } \\
(\mathrm{g})\end{array}$ & $\begin{array}{c}\text { U-238 } \\
(\mathrm{g})\end{array}$ & $\begin{array}{c}\text { U-235 } \\
(\mathrm{g})\end{array}$ & $\begin{array}{c}\text { Mo } \\
(\mathrm{g})\end{array}$ \\
\hline A-1 & L1B31Z & 26.864 & 21.513 & 5.351 & 2.945 \\
\hline A-2 & L1B33Z & 28.242 & 22.619 & 5.623 & 3.065 \\
\hline A-3 & L1B32Z & 26.448 & 21.180 & 5.269 & 2.900 \\
\hline A-4 & L1B51Z & 27.758 & 22.231 & 5.527 & 3.012 \\
\hline A-5 & L1B52Z & 28.092 & 22.499 & 5.593 & 3.049 \\
\hline A-6 & L1B53Z & 28.453 & 22.788 & 5.665 & 3.088 \\
\hline B-1 & L1H34Z & 27.697 & 22.180 & 5.517 & 3.036 \\
\hline B-2 & L1H35Z & 27.755 & 22.226 & 5.529 & 3.043 \\
\hline B-3 & L1H36Z & 27.503 & 22.024 & 5.479 & 3.015 \\
\hline B-4 & L1H37Z & 27.467 & 21.998 & 5.469 & 2.981 \\
\hline B-5 & L1H38Z & 27.983 & 22.411 & 5.571 & 3.037 \\
\hline B-6 & L1H39Z & 27.918 & 22.359 & 5.558 & 3.030 \\
\hline
\end{tabular}

Table 3: AFIP-4 constituent densities.

\begin{tabular}{|c|c|c|c|c|c|c|}
\hline $\begin{array}{c}\text { Fuel Plate } \\
\text { Location }\end{array}$ & $\begin{array}{c}\text { Fuel } \\
\text { Plate ID }\end{array}$ & $\begin{array}{c}\text { Fuel Meat } \\
\text { Volume } \\
(\mathrm{cc})\end{array}$ & $\begin{array}{c}\text { Total-U } \\
(\mathrm{g} / \mathrm{cc})\end{array}$ & $\begin{array}{c}\text { U-238 } \\
(\mathrm{g} / \mathrm{cc})\end{array}$ & $\begin{array}{c}\text { U-235 } \\
(\mathrm{g} / \mathrm{cc})\end{array}$ & $\begin{array}{c}\text { Mo } \\
(\mathrm{g} / \mathrm{cc})\end{array}$ \\
\hline A-1 & L1B31Z & 1.92 & 14.015 & 11.224 & 2.792 & 1.536 \\
\hline A-2 & L1B33Z & 1.87 & 15.095 & 12.090 & 3.005 & 1.638 \\
\hline A-3 & L1B32Z & 2.06 & 12.844 & 10.286 & 2.559 & 1.408 \\
\hline A-4 & L1B51Z & 1.90 & 14.619 & 11.708 & 2.911 & 1.586 \\
\hline A-5 & L1B52Z & 1.88 & 14.920 & 11.949 & 2.970 & 1.619 \\
\hline A-6 & L1B53Z & 1.93 & 14.772 & 11.831 & 2.941 & 1.603 \\
\hline B-1 & L1H34Z & 1.94 & 14.290 & 11.444 & 2.846 & 1.566 \\
\hline B-2 & L1H35Z & 1.94 & 14.320 & 11.467 & 2.853 & 1.570 \\
\hline B-3 & L1H36Z & 1.89 & 14.516 & 11.624 & 2.892 & 1.591 \\
\hline B-4 & L1H37Z & 1.87 & 14.703 & 11.775 & 2.928 & 1.596 \\
\hline B-5 & L1H38Z & 2.00 & 13.965 & 11.184 & 2.780 & 1.516 \\
\hline B-6 & L1H39Z & 1.92 & 14.573 & 11.672 & 2.901 & 1.582 \\
\hline
\end{tabular}




\section{EXPERIMENT HARDWARE}

The experiment hardware list for AFIP-4 is that used for all of the AFIP experiments with the exception of the plate frame assembly and the fuel plates. The drawing numbers and titles are in Table 4.

Table 4. AFIP-4 Irradiation Hardware Drawing List.

\begin{tabular}{c|c}
\hline Drawing Number & Drawing Title \\
\hline 635791 & $\begin{array}{c}\text { ATR Full Size Plate in Center Flux Trap Position (AFIP) Flux } \\
\text { Monitor Wire Holder Assembly Details }\end{array}$ \\
\hline 635792 & $\begin{array}{c}\text { ATR Full Size Plate in Center Flux Trap Position (AFIP) Plate } \\
\text { Holder Tube Assembly and Details }\end{array}$ \\
\hline 635793 & ATR Full Size Plate in Center Flux Trap Position (AFIP) Test \\
Train Assembly
\end{tabular}

The AFIP test train assembly as shown in Figure 2 shows the main components of the test assembly, which includes the flux monitor wire holders, the ram and ramrod, the frame assembly and the fuel plates. Figure 3 shows the test train assembly with the retriever attached to the top. The retriever is used to get the test train assembly out of the reactor. The flux monitor wire holders house the flux monitor wires. The ram is used to compress the rails of the fuel plate frame assemblies to minimize vibrations and hold the fuel plate frame assemblies in place. The ramrod is the last component to be inserted; it is used to push the ram against the fuel plate frame assembly. The frame assembly holds the fuel plates into place and is shown in Figure 4. Figure 5 has the specific fuel plate dimensions and nominal fuel foil dimensions. Figure 6 is a radial cross section of the test train assembly and shows the locations of all the components. 


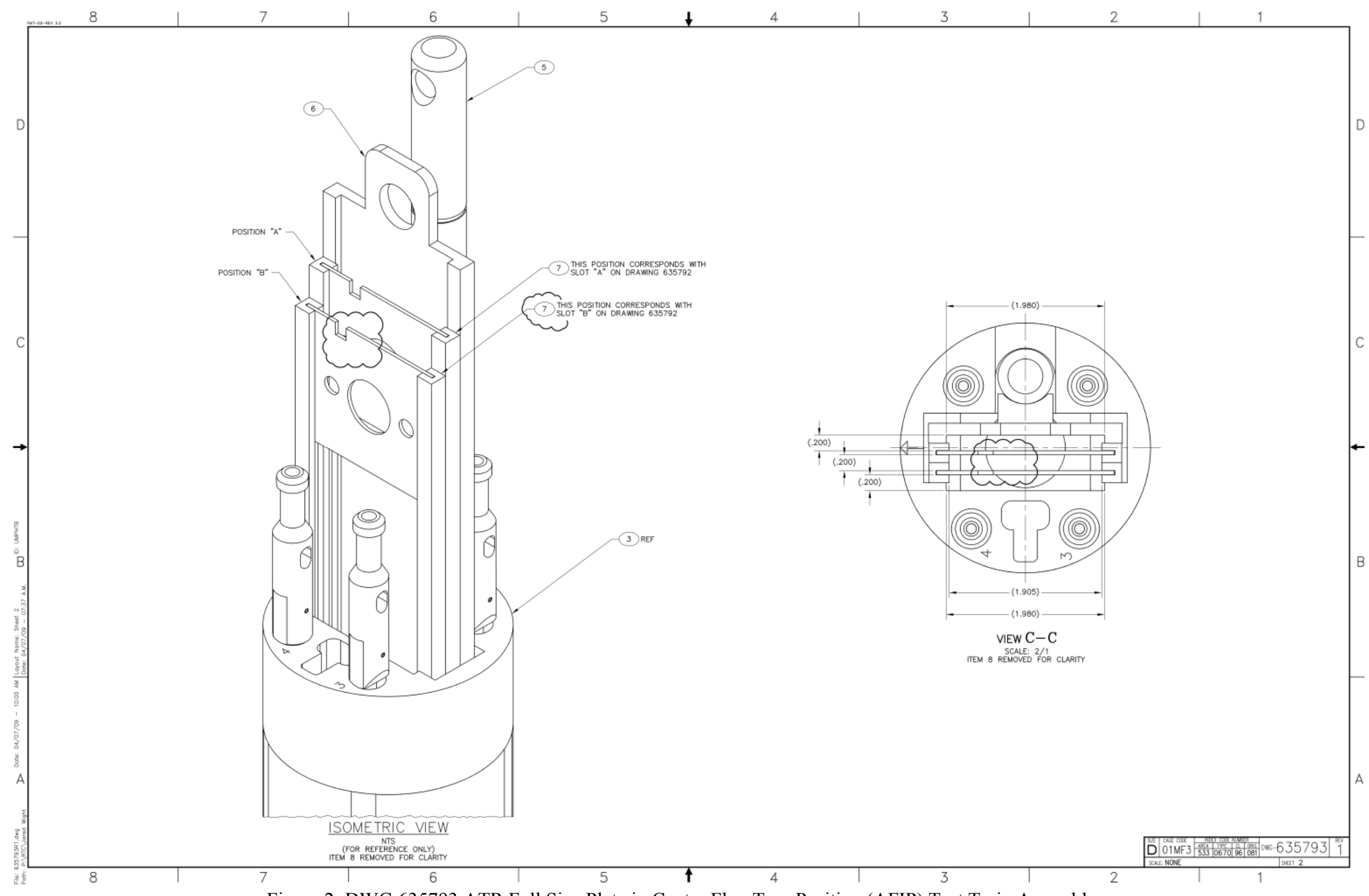

Figure 2: DWG 635793 ATR Full Size Plate in Center Flux Trap Position (AFIP) Test Train Assembly. 


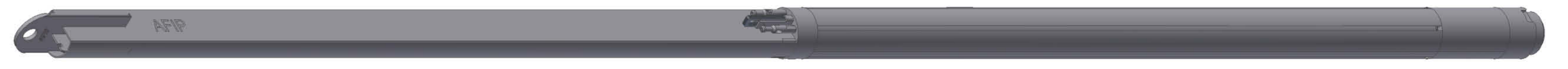

3

Figure 3: Isometric view of the AFIP-4 Test Train Assembly. 


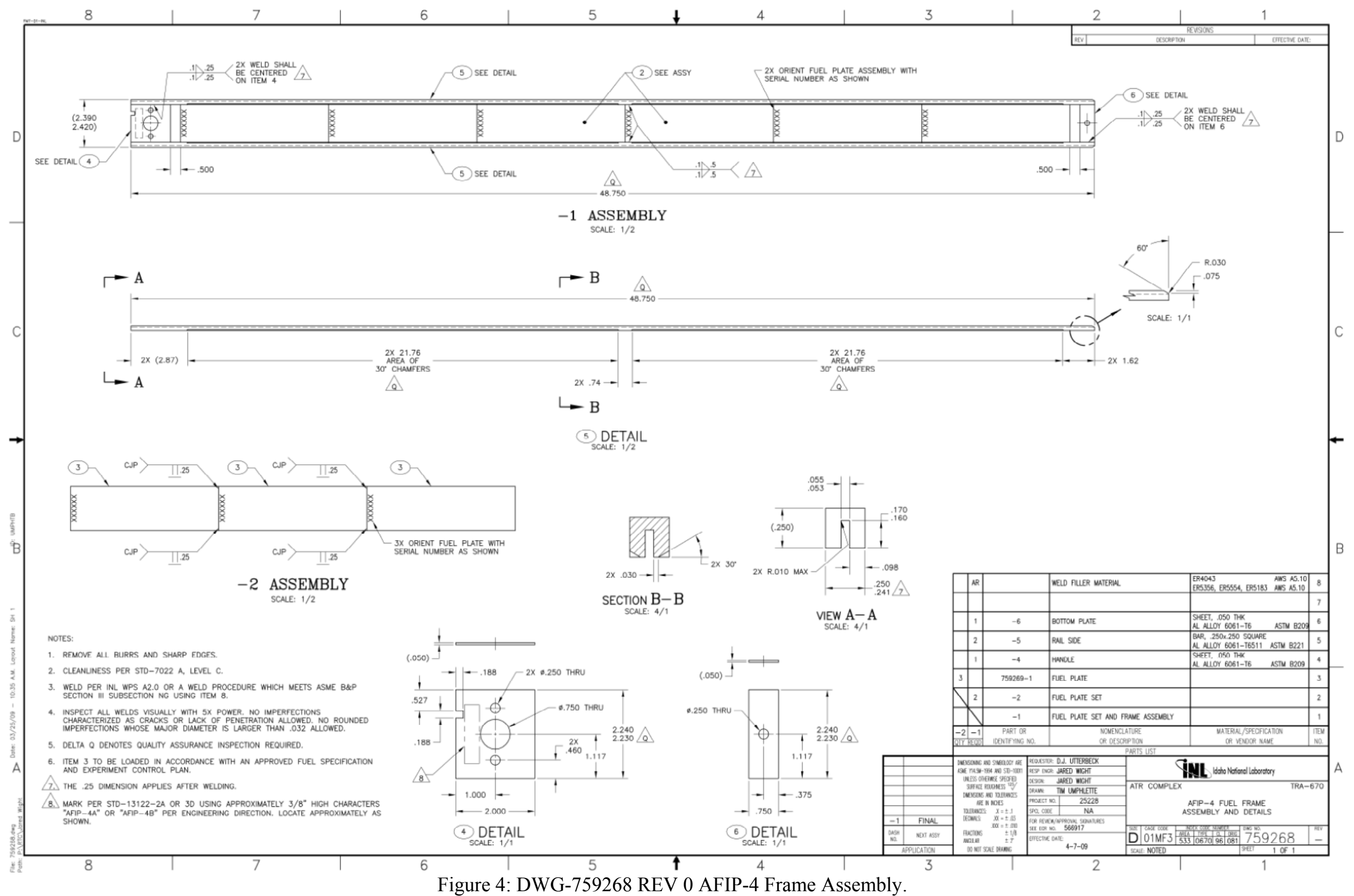




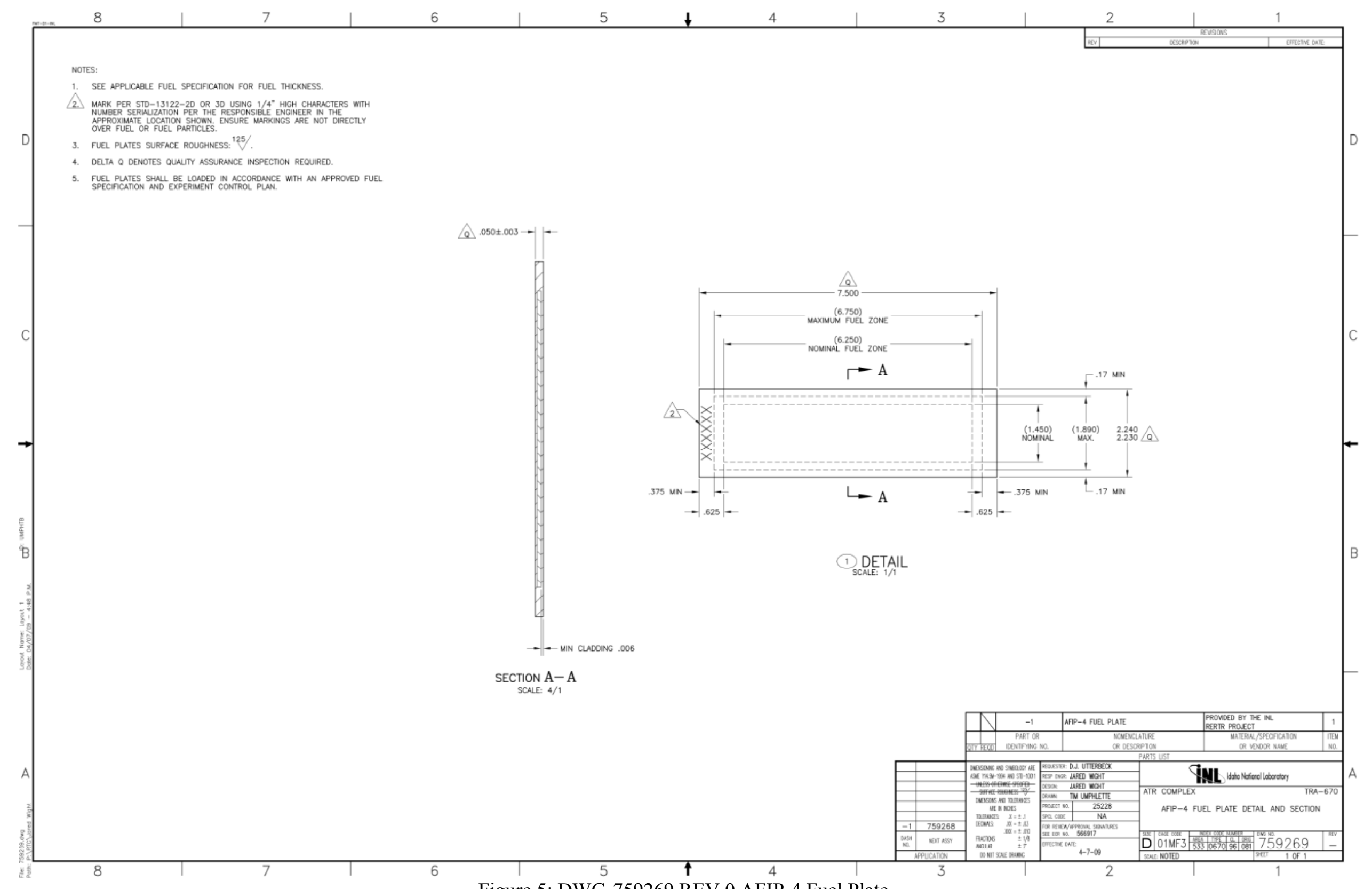

Figure 5: DWG-759269 REV 0 AFIP-4 Fuel Plate. 


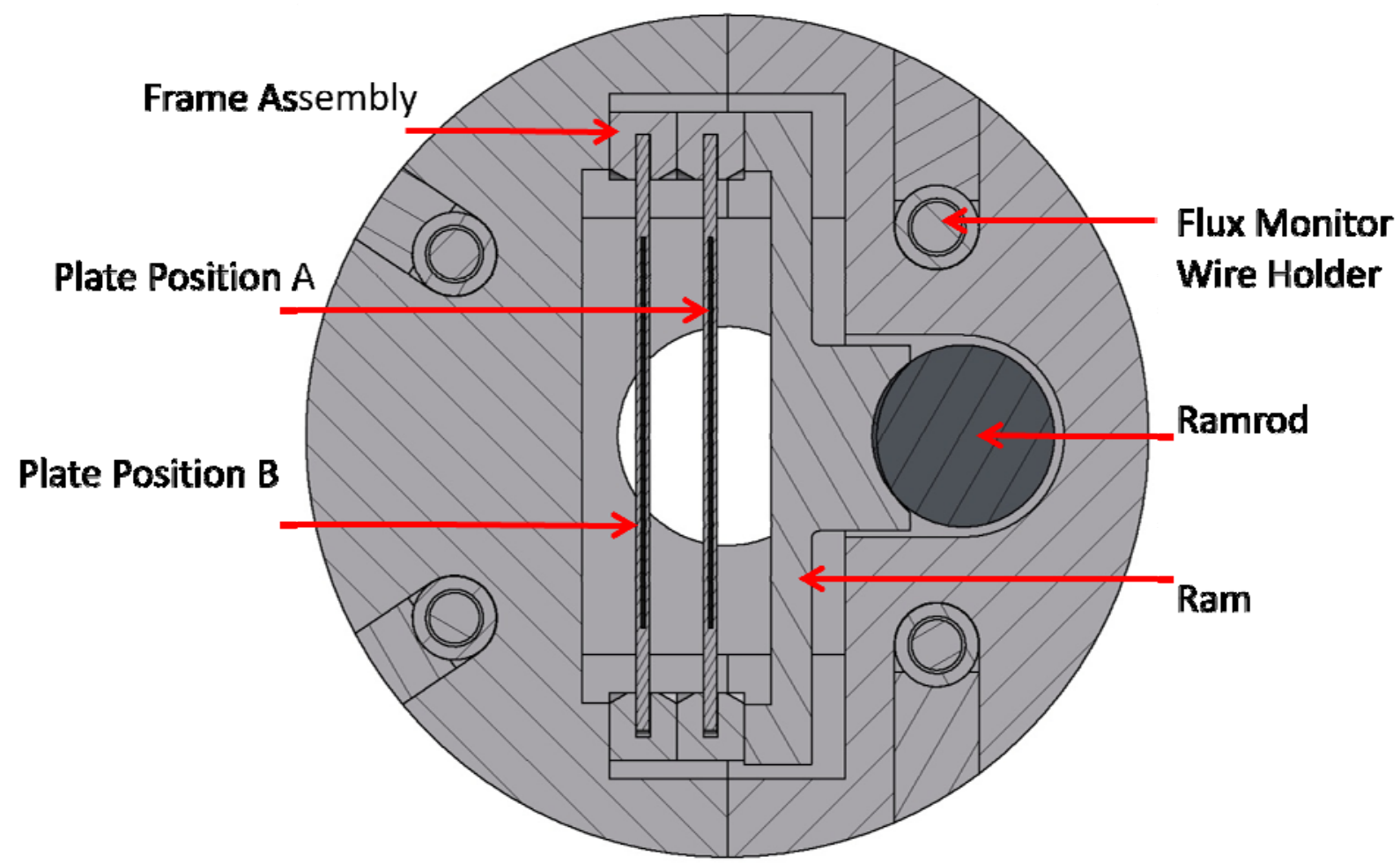

Figure 6: Radial Cross Section of the AFIP Test Train Assembly. 


\section{SAFETY ANALYSIS}

The safety analysis that was performed on the AFIP-4 experiment includes thermal/hydraulic analysis, physics analysis, and structural analysis. The following table (Table 5) summarizes the safety analyses performed on the AFIP-4 experiment.

Table 5: Summary table of the safety analyses done for the AFIP-4 experiment.

\begin{tabular}{|c|l|}
\hline ECAR Number & \multicolumn{1}{c|}{ Description } \\
\hline ECAR-543 & $\begin{array}{l}\text { ATR Physics Analysis of the AFIP-4 Experiment in the Center } \\
\text { Flux Trap }\end{array}$ \\
\hline ECAR-255 & $\begin{array}{l}\text { Analysis of the Effects of the ATR Full-Size Plate in Center Flux } \\
\text { Trap Position (AFIP) 4 Fueled Plate Test on the ATRC Axial Flux } \\
\text { Profile }\end{array}$ \\
\hline ECAR-59, Rev. 2 & $\begin{array}{l}\text { Temperature Reactivity Coefficients for the AFIP Experiment in } \\
\text { the Center Flux Trap of ATR }\end{array}$ \\
\hline TEV-485, Rev. 1 & AFIP-4 Thermal Evaluation \\
\hline ECAR-522 & AFIP-4 Fuel Plate Assembly Structural Evaluation \\
\hline ECAR-121 & Total Flow Evaluations for AFIP and Backups \\
\hline ECAR-126 & RELAP Analysis for AFIP-2 Flow Restrictor \\
\hline ECAR-432, Rev. 1 & $\begin{array}{l}\text { Four Plate AFIP Experiment SIPT>1/2 Inch Pump Discharge Break } \\
\text { Reactivity Transient Analysis }\end{array}$ \\
\hline
\end{tabular}




\section{IRRADIATION HISTORY}

The AFIP-4 test assembly was irradiated during cycles 144B and 145A in the ATR Center Flux Trap (CFT). Cycle 144B ran for 51.7 effective full power days (EFPDs) with an average center lobe power of 22.4 MW (total core power of 104.5 MW). There was one mid-cycle SCRAM during cycle 144B with duration of 3 days. Cycle 145A ran for 54.7 EFPDs with an average center lobe power of 23.2 MW (total core power of $108.6 \mathrm{MW}$ ). There were three mid-cycle SCRAMs during cycle 145A with duration of 2 days, 2 days and 3 days, respectively for a total of 7 days. This information is summarized in Table 6 .

Table 6: Irradiation history for AFIP-4.

\begin{tabular}{|c|c|c|c|c|c|c|c|c|}
\hline ATR & AFIP & $\begin{array}{c}\text { AFIP-4 } \\
\text { Frames } \\
\text { Irradiated }\end{array}$ & $\begin{array}{c}\text { Dates } \\
\text { Irradiated }\end{array}$ & $\begin{array}{c}\text { Cycle } \\
\text { EFPDs }\end{array}$ & $\begin{array}{c}\text { Mid-Cycle } \\
\text { Scram } \\
\text { Decay } \\
\text { Days }\end{array}$ & $\begin{array}{c}\text { Post-Cycle } \\
\text { Decay } \\
\text { Days }\end{array}$ & $\begin{array}{c}\text { Fenter } \\
\text { Trap } \\
\text { Power } \\
\text { (MW) }\end{array}$ & $\begin{array}{c}\text { Total } \\
\text { Core } \\
\text { Power } \\
\text { (MW) }\end{array}$ \\
\hline 144B & AFIP-4 & A,B & $\begin{array}{c}5 / 11 / 2009- \\
7 / 4 / 2009\end{array}$ & 51.7 & 3 & 63 & 22.4 & 104.5 \\
\hline $145 \mathrm{~A}$ & AFIP-4 & A,B & $\begin{array}{c}9 / 5 / 2009- \\
11 / 6 / 2009\end{array}$ & 54.7 & 7 & 18 & 23.2 & 108.6 \\
\hline
\end{tabular}

The power history for each cycle is obtained as an ATR Surveillance Report from the ATR Data Acquisition System (DAS). The plots of each lobe power on an hourly basis are shown in Figure 7 for Cycle 144B and Figure 8 for Cycle 145A.

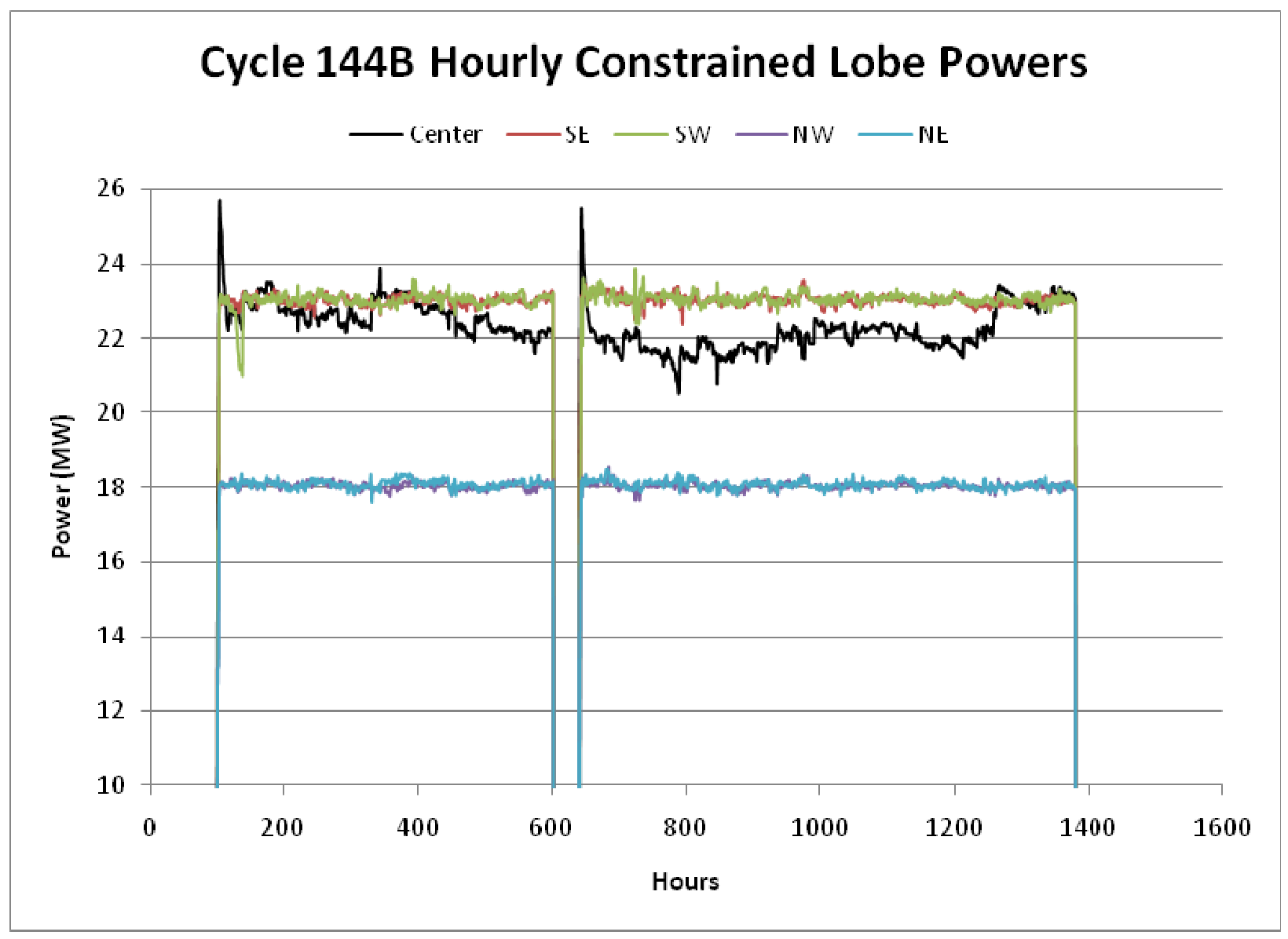

Figure 7: Hourly constrained lobe power history for Cycle 144B. 


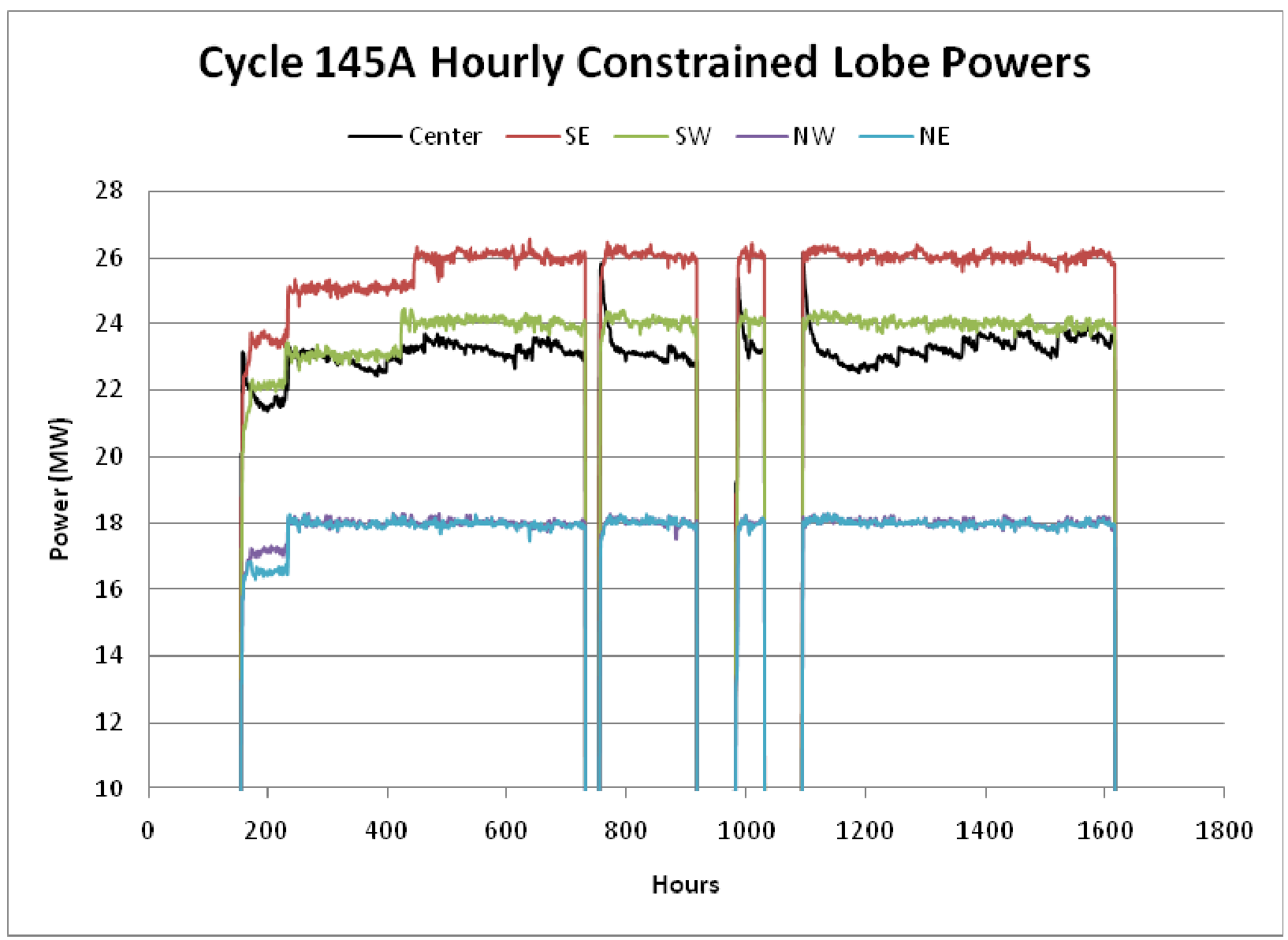

Figure 8: Hourly constrained lobe power history for Cycle 145A. 


\section{AS-RUN NUCLEAR ANALYSIS}

\subsection{Neutronics}

The as-run calculations were performed using the irradiation history in Table 6, the Monte Carlo N-Particle (MCNP) code, ORIGEN2.2 and $\mathrm{MCWO}^{3}$. The calculated as-run fission heat rates, fission densities, and as-run U-235 burnup results for the fueled plates reported have an uncertainty band $(1 \sigma)$ of $2.5 \%{ }^{3}$. The time intervals used to calculate the average plate power and burnup is shown in Table 7.

Table 7: Cycle breakdown.

\begin{tabular}{ccc}
\hline $\begin{array}{c}\text { Time } \\
\text { Interval }\end{array}$ & $\begin{array}{c}\text { 144B } \\
\text { (days) }\end{array}$ & $\begin{array}{c}145 \mathrm{~A} \\
\text { (days) }\end{array}$ \\
\hline BOC & 0.0 & 0.0 \\
MOC 1 & 18.0 & 18.0 \\
MOC 2 & 20.0 & 20.0 \\
EOC & 13.7 & 16.7 \\
\hline Total EFPDs & 51.7 & 54.7 \\
\hline \hline Cumulative & 51.7 & 106.4 \\
\hline
\end{tabular}

The MCNP-calculated average plate power and burnup for the time intervals for each cycle are shown in Table 8 through Table 23. The plots of the fission power density and fission density as a function of the ATR Cycle time interval are in Appendix A. 
Table 8: Cycle 144B, MCNP-Calculated HGRs and Neutron Flux for AFIP-4 Fuel Foils in Position A, 0 EFPD (BOC) Center Lobe Power at 22.4 MW. ${ }^{3}$

\begin{tabular}{|c|c|c|c|c|c|c|}
\hline $\begin{array}{c}\text { Plate } \\
\text { ID }\end{array}$ & $\begin{array}{l}\text { Fuel } \\
\text { Plate }\end{array}$ & $\begin{array}{c}\text { MCNP } \\
\text { Elevation }^{a} \\
\text { (inches) }^{2}\end{array}$ & $\begin{array}{l}\text { Fission } \\
\text { Power } \\
\text { Density } \\
\text { (W/cc) }\end{array}$ & $\begin{array}{c}\text { Fission } \\
\text { Heat } \\
\text { Rate } \\
(\mathrm{W} / \mathrm{g}) \\
\end{array}$ & $\begin{array}{c}\text { Surface } \\
\text { Heat } \\
\text { Flux } \\
(W / c m 2) \\
\end{array}$ & $\begin{array}{c}\text { Neutron Flux } \\
(\mathrm{n} / \mathrm{cm} 2 \mathrm{sec})\end{array}$ \\
\hline \multirow{5}{*}{ A-1 } & \multirow{5}{*}{ L1B31Z } & 21.14 & $6.92 \mathrm{E}+03$ & 433.81 & 114.24 & $4.38 \mathrm{E}+14$ \\
\hline & & 19.90 & $8.20 \mathrm{E}+03$ & 513.98 & 135.35 & $5.18 \mathrm{E}+14$ \\
\hline & & 18.66 & $9.25 \mathrm{E}+03$ & 579.63 & 152.64 & $5.90 \mathrm{E}+14$ \\
\hline & & 17.42 & $1.04 \mathrm{E}+04$ & 653.38 & 172.06 & $6.67 \mathrm{E}+14$ \\
\hline & & 16.18 & $1.20 \mathrm{E}+04$ & 749.67 & 197.42 & $7.27 \mathrm{E}+14$ \\
\hline \multirow{5}{*}{ A-2 } & \multirow{5}{*}{ L1B33Z } & 13.89 & $1.45 \mathrm{E}+04$ & 844.03 & 239.63 & $8.24 \mathrm{E}+14$ \\
\hline & & 12.65 & $1.48 \mathrm{E}+04$ & 860.39 & 244.28 & $8.73 E+14$ \\
\hline & & 11.41 & $1.57 \mathrm{E}+04$ & 915.61 & 259.95 & $9.22 \mathrm{E}+14$ \\
\hline & & 10.17 & $1.66 \mathrm{E}+04$ & 963.86 & 273.65 & $9.55 \mathrm{E}+14$ \\
\hline & & 8.93 & $1.79 \mathrm{E}+04$ & 1040.85 & 295.51 & $9.91 \mathrm{E}+14$ \\
\hline \multirow{5}{*}{ A-3 } & \multirow{5}{*}{ L1B32Z } & 6.63 & $1.66 \mathrm{E}+04$ & 1137.20 & 274.77 & $1.02 \mathrm{E}+15$ \\
\hline & & 5.39 & $1.63 \mathrm{E}+04$ & 1113.22 & 268.98 & $1.04 \mathrm{E}+15$ \\
\hline & & 4.15 & $1.67 \mathrm{E}+04$ & 1137.87 & 274.93 & $1.05 \mathrm{E}+15$ \\
\hline & & 2.91 & $1.68 \mathrm{E}+04$ & 1149.01 & 277.62 & $1.06 \mathrm{E}+15$ \\
\hline & & 1.68 & $1.75 \mathrm{E}+04$ & 1195.09 & 288.76 & $1.06 \mathrm{E}+15$ \\
\hline \multirow{5}{*}{$A-4$} & \multirow{5}{*}{ L1B51Z } & -1.68 & $1.93 \mathrm{E}+04$ & 1161.59 & 319.04 & $1.07 \mathrm{E}+15$ \\
\hline & & -2.91 & $1.84 \mathrm{E}+04$ & 1106.25 & 303.84 & $1.07 \mathrm{E}+15$ \\
\hline & & -4.15 & $1.84 \mathrm{E}+04$ & 1104.54 & 303.37 & $1.06 \mathrm{E}+15$ \\
\hline & & -5.39 & $1.83 \mathrm{E}+04$ & 1097.71 & 301.50 & $1.06 \mathrm{E}+15$ \\
\hline & & -6.63 & $1.87 \mathrm{E}+04$ & 1125.09 & 309.02 & $1.05 \mathrm{E}+15$ \\
\hline \multirow{5}{*}{$A-5$} & \multirow{5}{*}{ L1B52Z } & -8.93 & $1.85 \mathrm{E}+04$ & 1088.82 & 305.71 & $1.01 \mathrm{E}+15$ \\
\hline & & -10.17 & $1.72 \mathrm{E}+04$ & 1012.47 & 284.27 & $9.94 \mathrm{E}+14$ \\
\hline & & -11.41 & $1.66 \mathrm{E}+04$ & 975.47 & 273.88 & $9.73 \mathrm{E}+14$ \\
\hline & & -12.65 & $1.58 \mathrm{E}+04$ & 929.85 & 261.07 & $9.30 \mathrm{E}+14$ \\
\hline & & -13.89 & $1.56 \mathrm{E}+04$ & 915.91 & 257.16 & $8.95 \mathrm{E}+14$ \\
\hline \multirow{5}{*}{ A-6 } & \multirow{5}{*}{ L1B53Z } & -16.18 & $1.38 \mathrm{E}+04$ & 824.69 & 228.57 & $8.10 \mathrm{E}+14$ \\
\hline & & -17.42 & $1.21 \mathrm{E}+04$ & 722.35 & 200.21 & $7.40 \mathrm{E}+14$ \\
\hline & & -18.66 & $1.10 \mathrm{E}+04$ & 652.75 & 180.92 & $6.77 \mathrm{E}+14$ \\
\hline & & -19.90 & $9.94 \mathrm{E}+03$ & 591.86 & 164.04 & $6.08 \mathrm{E}+14$ \\
\hline & & -21.14 & $8.61 \mathrm{E}+03$ & 513.12 & 142.22 & $5.18 \mathrm{E}+14$ \\
\hline
\end{tabular}

(a) Elevations for MCNP cells are reported at cell centroids relative to the modeled core axial midplane at 24.0 inches. 
Table 9: Cycle 144B, MCNP-Calculated HGRs and Neutron Flux for AFIP-4 Fuel Foils in Position B, 0 EFPD (BOC), Center Lobe Power at 22.4 MW. ${ }^{3}$

\begin{tabular}{|c|c|c|c|c|c|c|}
\hline $\begin{array}{c}\text { Plate } \\
\text { ID }\end{array}$ & $\begin{array}{l}\text { Fuel } \\
\text { Plate } \\
\end{array}$ & $\begin{array}{c}\text { MCNP } \\
\text { Elevation }^{a} \\
\text { (inches) }^{\text {(inche }}\end{array}$ & $\begin{array}{c}\text { Fission } \\
\text { Power } \\
\text { Density } \\
\text { (W/cc) } \\
\end{array}$ & $\begin{array}{c}\text { Fission } \\
\text { Heat } \\
\text { Rate } \\
\text { (W/g) } \\
\end{array}$ & $\begin{array}{c}\text { Surface } \\
\text { Heat } \\
\text { Flux } \\
(W / c m 2) \\
\end{array}$ & $\begin{array}{c}\text { Neutron Flux } \\
(\mathrm{n} / \mathrm{cm} 2 \mathrm{sec})\end{array}$ \\
\hline \multirow{5}{*}{ B-1 } & \multirow{5}{*}{ L1H34Z } & 21.14 & $6.88 \mathrm{E}+03$ & 424.36 & 113.55 & $4.31 \mathrm{E}+14$ \\
\hline & & 19.90 & $8.05 \mathrm{E}+03$ & 496.56 & 132.87 & $5.14 \mathrm{E}+14$ \\
\hline & & 18.66 & $9.20 \mathrm{E}+03$ & 567.51 & 151.86 & $5.87 \mathrm{E}+14$ \\
\hline & & 17.42 & $1.03 \mathrm{E}+04$ & 636.68 & 170.37 & $6.62 \mathrm{E}+14$ \\
\hline & & 16.18 & $1.19 \mathrm{E}+04$ & 734.61 & 196.57 & $7.21 \mathrm{E}+14$ \\
\hline \multirow{5}{*}{ B-2 } & \multirow{5}{*}{ L1H35Z } & 13.89 & $1.35 \mathrm{E}+04$ & 831.12 & 222.83 & $8.22 \mathrm{E}+14$ \\
\hline & & 12.65 & $1.37 \mathrm{E}+04$ & 844.55 & 226.43 & $8.70 \mathrm{E}+14$ \\
\hline & & 11.41 & $1.47 \mathrm{E}+04$ & 902.40 & 241.94 & $9.08 \mathrm{E}+14$ \\
\hline & & 10.17 & $1.54 \mathrm{E}+04$ & 947.12 & 253.93 & $9.51 \mathrm{E}+14$ \\
\hline & & 8.93 & $1.67 \mathrm{E}+04$ & 1029.18 & 275.93 & $9.84 \mathrm{E}+14$ \\
\hline \multirow{5}{*}{ B-3 } & \multirow{5}{*}{ L1H36Z } & 6.63 & $1.80 \mathrm{E}+04$ & 1088.24 & 296.37 & $1.02 \mathrm{E}+15$ \\
\hline & & 5.39 & $1.74 \mathrm{E}+04$ & 1056.85 & 287.82 & $1.03 \mathrm{E}+15$ \\
\hline & & 4.15 & $1.77 \mathrm{E}+04$ & 1071.12 & 291.71 & $1.05 \mathrm{E}+15$ \\
\hline & & 2.91 & $1.79 \mathrm{E}+04$ & 1082.16 & 294.71 & $1.06 \mathrm{E}+15$ \\
\hline & & 1.68 & $1.90 \mathrm{E}+04$ & 1153.24 & 314.07 & $1.07 \mathrm{E}+15$ \\
\hline \multirow{5}{*}{ B-4 } & \multirow{5}{*}{ L1H37Z } & -1.68 & $1.91 \mathrm{E}+04$ & 1144.45 & 314.84 & $1.06 \mathrm{E}+15$ \\
\hline & & -2.91 & $1.81 \mathrm{E}+04$ & 1086.17 & 298.81 & $1.07 \mathrm{E}+15$ \\
\hline & & -4.15 & $1.80 \mathrm{E}+04$ & 1078.52 & 296.70 & $1.06 \mathrm{E}+15$ \\
\hline & & -5.39 & $1.78 \mathrm{E}+04$ & 1066.39 & 293.36 & $1.05 \mathrm{E}+15$ \\
\hline & & -6.63 & $1.84 \mathrm{E}+04$ & 1102.51 & 303.30 & $1.04 \mathrm{E}+15$ \\
\hline \multirow{5}{*}{ B-5 } & \multirow{5}{*}{ L1H38Z } & -8.93 & $1.68 \mathrm{E}+04$ & 1060.79 & 277.69 & $1.01 \mathrm{E}+15$ \\
\hline & & -10.17 & $1.58 \mathrm{E}+04$ & 995.27 & 260.53 & $9.86 \mathrm{E}+14$ \\
\hline & & -11.41 & $1.53 \mathrm{E}+04$ & 963.81 & 252.30 & $9.59 \mathrm{E}+14$ \\
\hline & & -12.65 & $1.46 \mathrm{E}+04$ & 919.22 & 240.63 & $9.22 \mathrm{E}+14$ \\
\hline & & -13.89 & $1.45 \mathrm{E}+04$ & 911.71 & 238.66 & $8.85 E+14$ \\
\hline \multirow{5}{*}{ B-6 } & \multirow{5}{*}{ L1H39Z } & -16.18 & $1.33 \mathrm{E}+04$ & 805.57 & 219.32 & $7.97 \mathrm{E}+14$ \\
\hline & & -17.42 & $1.16 \mathrm{E}+04$ & 706.45 & 192.33 & $7.33 \mathrm{E}+14$ \\
\hline & & -18.66 & $1.06 \mathrm{E}+04$ & 642.85 & 175.01 & $6.72 \mathrm{E}+14$ \\
\hline & & -19.90 & $9.64 \mathrm{E}+03$ & 584.84 & 159.22 & $6.07 \mathrm{E}+14$ \\
\hline & & -21.14 & $8.29 \mathrm{E}+03$ & 502.47 & 136.80 & $5.14 \mathrm{E}+14$ \\
\hline
\end{tabular}

(a) Elevations for MCNP cells are reported at cell centroids relative to the modeled core axial midplane at 24.0 inches. 
Table 10: Cycle 144B, MCNP-Calculated HGR, Neutron Flux, Depletion, and Fission Density for AFIP-4 Fuel Foils in Position A, 18.0 EFPD (MOC1), Center Lobe Power at 22.4 MW (18EFPD Cumulative). ${ }^{3}$

\begin{tabular}{|c|c|c|c|c|c|c|c|c|}
\hline $\begin{array}{c}\text { Plate } \\
\text { ID }\end{array}$ & $\begin{array}{l}\text { Fuel } \\
\text { Plate } \\
\end{array}$ & $\begin{array}{c}\text { MCNP } \\
\text { Elevation }^{a} \\
\text { (inches) }^{a}\end{array}$ & $\begin{array}{l}\text { Fission } \\
\text { Power } \\
\text { Density } \\
\text { (W/cc) } \\
\end{array}$ & $\begin{array}{c}\text { Fission } \\
\text { Heat } \\
\text { Rate } \\
(W / g) \\
\end{array}$ & $\begin{array}{c}\text { Surface } \\
\text { Heat } \\
\text { Flux } \\
(\mathbf{W} / \mathbf{c m} 2) \\
\end{array}$ & $\begin{array}{c}\begin{array}{c}\text { Neutron } \\
\text { Flux } \\
\text { (n/cm2sec) }\end{array} \\
\end{array}$ & $\begin{array}{c}\% \\
\text { Depletion } \\
\text { U-235 } \\
(\%) \\
\end{array}$ & $\begin{array}{c}\text { Fission } \\
\text { Density } \\
\text { (fissions/cc) }\end{array}$ \\
\hline \multirow{5}{*}{ A-1 } & \multirow{5}{*}{ L1B31Z } & 21.14 & $6.74 \mathrm{E}+03$ & 425.80 & 111.25 & $4.43 \mathrm{E}+14$ & $5.64 \%$ & $3.47 \mathrm{E}+20$ \\
\hline & & 19.90 & $7.81 \mathrm{E}+03$ & 494.55 & 129.02 & $5.20 \mathrm{E}+14$ & $6.65 \%$ & $4.11 \mathrm{E}+20$ \\
\hline & & 18.66 & $8.93 \mathrm{E}+03$ & 565.63 & 147.38 & $5.96 \mathrm{E}+14$ & $7.50 \%$ & $4.64 \mathrm{E}+20$ \\
\hline & & 17.42 & $9.87 \mathrm{E}+03$ & 626.15 & 162.92 & $6.67 \mathrm{E}+14$ & $8.42 \%$ & $5.23 \mathrm{E}+20$ \\
\hline & & 16.18 & $1.13 \mathrm{E}+04$ & 719.18 & 186.83 & $7.36 \mathrm{E}+14$ & $9.51 \%$ & $6.00 \mathrm{E}+20$ \\
\hline \multirow{5}{*}{ A-2 } & \multirow{5}{*}{ L1B33Z } & 13.89 & $1.36 \mathrm{E}+04$ & 802.87 & 224.47 & $8.41 \mathrm{E}+14$ & $10.69 \%$ & $7.28 \mathrm{E}+20$ \\
\hline & & 12.65 & $1.40 \mathrm{E}+04$ & 829.81 & 231.91 & $8.88 \mathrm{E}+14$ & $10.93 \%$ & $7.42 \mathrm{E}+20$ \\
\hline & & 11.41 & $1.49 \mathrm{E}+04$ & 879.57 & 245.59 & $9.31 \mathrm{E}+14$ & $11.55 \%$ & $7.90 \mathrm{E}+20$ \\
\hline & & 10.17 & $1.54 \mathrm{E}+04$ & 913.80 & 254.96 & $9.67 \mathrm{E}+14$ & $12.09 \%$ & $8.31 \mathrm{E}+20$ \\
\hline & & 8.93 & $1.67 \mathrm{E}+04$ & 992.06 & 276.42 & $9.97 \mathrm{E}+14$ & $13.03 \%$ & $8.98 \mathrm{E}+20$ \\
\hline \multirow{5}{*}{ A-3 } & \multirow{5}{*}{ L1B32Z } & 6.63 & $1.55 \mathrm{E}+04$ & 1079.75 & 255.62 & $1.04 \mathrm{E}+15$ & $14.10 \%$ & $8.35 \mathrm{E}+20$ \\
\hline & & 5.39 & $1.52 \mathrm{E}+04$ & 1062.47 & 251.64 & $1.05 \mathrm{E}+15$ & $13.82 \%$ & $8.17 \mathrm{E}+20$ \\
\hline & & 4.15 & $1.54 \mathrm{E}+04$ & 1074.37 & 254.32 & $1.06 \mathrm{E}+15$ & $14.10 \%$ & $8.35 \mathrm{E}+20$ \\
\hline & & 2.91 & $1.57 \mathrm{E}+04$ & 1093.07 & 258.74 & $1.07 \mathrm{E}+15$ & $14.23 \%$ & $8.43 \mathrm{E}+20$ \\
\hline & & 1.68 & $1.65 \mathrm{E}+04$ & 1151.80 & 272.42 & $1.08 \mathrm{E}+15$ & $14.75 \%$ & $8.77 \mathrm{E}+20$ \\
\hline \multirow{5}{*}{ A-4 } & \multirow{5}{*}{ L1B51Z } & -1.68 & $1.79 \mathrm{E}+04$ & 1100.82 & 296.12 & $1.08 \mathrm{E}+15$ & $14.37 \%$ & $9.69 \mathrm{E}+20$ \\
\hline & & -2.91 & $1.72 \mathrm{E}+04$ & 1056.79 & 284.55 & $1.08 \mathrm{E}+15$ & $13.72 \%$ & $9.23 \mathrm{E}+20$ \\
\hline & & -4.15 & $1.72 \mathrm{E}+04$ & 1057.16 & 284.63 & $1.08 \mathrm{E}+15$ & $13.72 \%$ & $9.21 \mathrm{E}+20$ \\
\hline & & -5.39 & $1.69 \mathrm{E}+04$ & 1033.49 & 278.28 & $1.07 \mathrm{E}+15$ & $13.64 \%$ & $9.16 \mathrm{E}+20$ \\
\hline & & -6.63 & $1.73 \mathrm{E}+04$ & 1061.52 & 285.70 & $1.06 \mathrm{E}+15$ & $13.96 \%$ & $9.39 \mathrm{E}+20$ \\
\hline \multirow{5}{*}{ A-5 } & \multirow{5}{*}{ L1B52Z } & -8.93 & $1.71 \mathrm{E}+04$ & 1027.82 & 282.96 & $1.03 \mathrm{E}+15$ & $13.61 \%$ & $9.28 \mathrm{E}+20$ \\
\hline & & -10.17 & $1.60 \mathrm{E}+04$ & 960.85 & 264.85 & $9.99 \mathrm{E}+14$ & $12.74 \%$ & $8.63 \mathrm{E}+20$ \\
\hline & & -11.41 & $1.55 \mathrm{E}+04$ & 925.78 & 255.35 & $9.72 \mathrm{E}+14$ & $12.26 \%$ & $8.32 \mathrm{E}+20$ \\
\hline & & -12.65 & $1.49 \mathrm{E}+04$ & 891.15 & 245.99 & $9.40 \mathrm{E}+14$ & $11.78 \%$ & $7.93 \mathrm{E}+20$ \\
\hline & & -13.89 & $1.44 \mathrm{E}+04$ & 861.20 & 237.81 & $8.96 \mathrm{E}+14$ & $11.55 \%$ & $7.81 \mathrm{E}+20$ \\
\hline \multirow{5}{*}{ A-6 } & \multirow{5}{*}{ L1B53Z } & -16.18 & $1.29 \mathrm{E}+04$ & 780.22 & 213.02 & $8.06 \mathrm{E}+14$ & $10.47 \%$ & $6.94 \mathrm{E}+20$ \\
\hline & & -17.42 & $1.16 \mathrm{E}+04$ & 700.59 & 191.65 & $7.50 \mathrm{E}+14$ & $9.20 \%$ & $6.08 \mathrm{E}+20$ \\
\hline & & -18.66 & $1.05 \mathrm{E}+04$ & 632.74 & 173.28 & $6.85 \mathrm{E}+14$ & $8.40 \%$ & $5.49 \mathrm{E}+20$ \\
\hline & & -19.90 & $9.51 \mathrm{E}+03$ & 572.71 & 157.03 & $6.13 \mathrm{E}+14$ & $7.59 \%$ & $4.98 \mathrm{E}+20$ \\
\hline & & -21.14 & $8.28 \mathrm{E}+03$ & 497.74 & 136.67 & $5.24 \mathrm{E}+14$ & $6.64 \%$ & $4.32 \mathrm{E}+20$ \\
\hline
\end{tabular}

(a) Elevations for MCNP cells are reported at cell centroids relative to the modeled core axial midplane at 24.0 inches. 
Table 11: Cycle 144B, MCNP-Calculated HGR, Neutron Flux, Depletion, and Fission Density for AFIP-4 Fuel Foils in Position B, 18 EFPD (MOC1), Center Lobe Power at 22.4 MW (18 EFPD Cumulative). ${ }^{3}$

\begin{tabular}{|c|c|c|c|c|c|c|c|c|}
\hline $\begin{array}{c}\text { Plate } \\
\text { ID }\end{array}$ & $\begin{array}{l}\text { Fuel } \\
\text { Plate } \\
\end{array}$ & $\begin{array}{c}\text { MCNP } \\
\text { Elevation }^{\mathrm{a}} \\
\text { (inches) }^{2}\end{array}$ & $\begin{array}{c}\text { Fission } \\
\text { Power } \\
\text { Density } \\
\text { (W/cc) } \\
\end{array}$ & $\begin{array}{c}\text { Fission } \\
\text { Heat } \\
\text { Rate } \\
(\mathbf{W} / \mathbf{g}) \\
\end{array}$ & $\begin{array}{c}\text { Surface } \\
\text { Heat } \\
\text { Flux } \\
(\mathbf{W} / \mathbf{c m} 2) \\
\end{array}$ & $\begin{array}{c}\text { Neutron } \\
\text { Flux } \\
\text { (n/cm2sec) } \\
\end{array}$ & $\begin{array}{c}\% \\
\text { Depletion } \\
\text { U-235 } \\
(\%) \\
\end{array}$ & $\begin{array}{c}\text { Fission } \\
\text { Density } \\
\text { (fissions/ce) }\end{array}$ \\
\hline \multirow{5}{*}{ B-1 } & \multirow{5}{*}{ L1H34Z } & 21.14 & $6.64 \mathrm{E}+03$ & 413.23 & 109.70 & $4.39 \mathrm{E}+14$ & $5.53 \%$ & $3.46 \mathrm{E}+20$ \\
\hline & & 19.90 & $7.72 \mathrm{E}+03$ & 480.63 & 127.43 & $5.18 \mathrm{E}+14$ & $6.43 \%$ & $4.05 \mathrm{E}+20$ \\
\hline & & 18.66 & $8.79 \mathrm{E}+03$ & 547.99 & 145.10 & $5.92 \mathrm{E}+14$ & $7.34 \%$ & $4.63 \mathrm{E}+20$ \\
\hline & & 17.42 & $9.88 \mathrm{E}+03$ & 616.85 & 163.12 & $6.65 \mathrm{E}+14$ & $8.24 \%$ & $5.20 \mathrm{E}+20$ \\
\hline & & 16.18 & $1.11 \mathrm{E}+04$ & 695.61 & 183.63 & $7.29 \mathrm{E}+14$ & $9.40 \%$ & $6.00 \mathrm{E}+20$ \\
\hline \multirow{5}{*}{ B-2 } & \multirow{5}{*}{ L1H35Z } & 13.89 & $1.28 \mathrm{E}+04$ & 801.42 & 211.62 & $8.33 \mathrm{E}+14$ & $10.55 \%$ & $6.80 \mathrm{E}+20$ \\
\hline & & 12.65 & $1.31 \mathrm{E}+04$ & 817.57 & 215.83 & $8.78 \mathrm{E}+14$ & $10.72 \%$ & $6.91 \mathrm{E}+20$ \\
\hline & & 11.41 & $1.38 \mathrm{E}+04$ & 861.70 & 227.24 & $9.18 \mathrm{E}+14$ & $11.46 \%$ & $7.38 \mathrm{E}+20$ \\
\hline & & 10.17 & $1.45 \mathrm{E}+04$ & .21 & .86 & $9.64 \mathrm{E}+14$ & $11.95 \%$ & $7.75 \mathrm{E}+20$ \\
\hline & & 8.93 & $1.56 \mathrm{E}+04$ & 977.42 & 257.18 & $9.88 \mathrm{E}+14$ & $12.95 \%$ & $8.42 \mathrm{E}+20$ \\
\hline \multirow{5}{*}{ B-3 } & \multirow{5}{*}{ L1H36Z } & 6.63 & $1.68 \mathrm{E}+04$ & 1038.06 & 277.25 & $1.03 \mathrm{E}+15$ & $13.57 \%$ & $9.04 \mathrm{E}+20$ \\
\hline & & 5.39 & $1.64 \mathrm{E}+04$ & 1014.18 & 270.98 & $1.05 \mathrm{E}+15$ & $13.24 \%$ & $8.78 \mathrm{E}+20$ \\
\hline & & 4.15 & $1.64 \mathrm{E}+04$ & 1016.51 & 271.52 & $1.05 \mathrm{E}+15$ & $13.41 \%$ & $8.90 \mathrm{E}+20$ \\
\hline & & 2.91 & $1.67 \mathrm{E}+04$ & 1031.42 & 275.45 & $1.06 \mathrm{E}+15$ & $13.57 \%$ & $8.99 \mathrm{E}+20$ \\
\hline & & 1.68 & $1.77 \mathrm{E}+04$ & 1092.68 & 291.50 & $1.08 \mathrm{E}+15$ & $14.31 \%$ & $9.58 \mathrm{E}+20$ \\
\hline \multirow{5}{*}{ B-4 } & \multirow{5}{*}{ L1H37Z } & -1.68 & $1.76 \mathrm{E}+04$ & 1081.19 & 291.38 & $1.08 \mathrm{E}+15$ & $14.19 \%$ & $9.60 \mathrm{E}+20$ \\
\hline & & -2.91 & $1.69 \mathrm{E}+04$ & 1035.21 & 279.26 & $1.08 \mathrm{E}+15$ & $13.56 \%$ & $9.11 \mathrm{E}+20$ \\
\hline & & -4.15 & $1.68 \mathrm{E}+04$ & 1029.44 & 277.74 & $1.07 \mathrm{E}+15$ & $13.48 \%$ & $9.05 \mathrm{E}+20$ \\
\hline & & -5.39 & $1.67 \mathrm{E}+04$ & 1022.99 & 276.03 & $1.06 \mathrm{E}+15$ & $13.32 \%$ & $8.95 \mathrm{E}+20$ \\
\hline & & -6.63 & $1.71 \mathrm{E}+04$ & 1046.24 & 282.17 & $1.06 \mathrm{E}+15$ & $13.72 \%$ & $9.25 \mathrm{E}+20$ \\
\hline \multirow{5}{*}{ B-5 } & \multirow{5}{*}{ L1H38Z } & -8.93 & $1.59 \mathrm{E}+04$ & 1025.09 & 263.23 & $1.02 \mathrm{E}+15$ & $13.27 \%$ & $8.47 \mathrm{E}+20$ \\
\hline & & -10.17 & $1.48 \mathrm{E}+04$ & 949.26 & 244.04 & $9.99 \mathrm{E}+14$ & $12.51 \%$ & $7.95 \mathrm{E}+20$ \\
\hline & & -11.41 & $1.43 \mathrm{E}+04$ & 915.22 & 235.43 & $9.71 \mathrm{E}+14$ & $12.17 \%$ & $7.70 \mathrm{E}+20$ \\
\hline & & -12.65 & $1.36 \mathrm{E}+04$ & 872.91 & 224.69 & $9.28 \mathrm{E}+14$ & $11.66 \%$ & $7.34 \mathrm{E}+20$ \\
\hline & & -13.89 & $1.35 \mathrm{E}+04$ & 867.68 & 223.39 & $8.96 \mathrm{E}+14$ & $11.50 \%$ & $7.28 \mathrm{E}+20$ \\
\hline \multirow{5}{*}{ B-6 } & \multirow{5}{*}{ L1H39Z } & -16.18 & $1.25 \mathrm{E}+04$ & 770.95 & 206.82 & $8.06 \mathrm{E}+14$ & $10.20 \%$ & $6.69 \mathrm{E}+20$ \\
\hline & & -17.42 & $1.12 \mathrm{E}+04$ & 686.68 & 184.54 & $7.39 \mathrm{E}+14$ & $9.07 \%$ & $5.87 \mathrm{E}+20$ \\
\hline & & -18.66 & $1.01 \mathrm{E}+04$ & 620.90 & 167.06 & $6.80 \mathrm{E}+14$ & $8.26 \%$ & $5.34 \mathrm{E}+20$ \\
\hline & & -19.90 & $9.16 \mathrm{E}+03$ & 561.69 & 151.28 & $6.12 \mathrm{E}+14$ & $7.54 \%$ & $4.86 \mathrm{E}+20$ \\
\hline & & -21.14 & $8.06 \mathrm{E}+03$ & 493.53 & 133.13 & $5.21 \mathrm{E}+14$ & $6.47 \%$ & $4.17 \mathrm{E}+20$ \\
\hline
\end{tabular}

(a) Elevations for MCNP cells are reported at cell centroids relative to the modeled core axial midplane at 24.0 inches. 
Table 12: Cycle 144B, MCNP-Calculated HGR, Neutron Flux, Depletion, and Fission Density for AFIP-4 Fuel Foils in Position A, 38 EFPD (MOC2), Center Lobe Power at 22.4 MW (38 EFPD Cumulative). ${ }^{3}$

\begin{tabular}{|c|c|c|c|c|c|c|c|c|}
\hline $\begin{array}{c}\text { Plate } \\
\text { ID } \\
\end{array}$ & $\begin{array}{c}\text { Fuel } \\
\text { Plate } \\
\end{array}$ & $\begin{array}{c}\text { MCNP } \\
\text { Elevation }^{a} \\
\text { (inches) }^{2}\end{array}$ & $\begin{array}{c}\text { Fission } \\
\text { Power } \\
\text { Density } \\
\text { (W/cc) } \\
\end{array}$ & $\begin{array}{c}\text { Fission } \\
\text { Heat } \\
\text { Rate } \\
(\mathrm{W} / \mathrm{g}) \\
\end{array}$ & $\begin{array}{c}\text { Surface } \\
\text { Heat } \\
\text { Flux } \\
(W / c m 2) \\
\end{array}$ & $\begin{array}{c}\text { Neutron } \\
\text { Flux } \\
\text { (n/cm2sec) } \\
\end{array}$ & $\begin{array}{c}\% \\
\text { Depletion } \\
\text { U-235 } \\
(\%) \\
\end{array}$ & $\begin{array}{c}\text { Fission } \\
\text { Density } \\
\text { (fissions/cc) }\end{array}$ \\
\hline \multirow{5}{*}{ A-1 } & \multirow{5}{*}{ L1B31Z } & 21.14 & $6.57 \mathrm{E}+03$ & 418.59 & 108.42 & $4.32 \mathrm{E}+14$ & $11.54 \%$ & $7.17 \mathrm{E}+20$ \\
\hline & & 19.90 & $7.59 \mathrm{E}+03$ & 484.92 & 125.25 & $5.06 \mathrm{E}+14$ & $13.47 \%$ & $8.40 \mathrm{E}+20$ \\
\hline & & 18.66 & $8.65 \mathrm{E}+03$ & 554.12 & 142.74 & $5.80 \mathrm{E}+14$ & $15.25 \%$ & $9.54 \mathrm{E}+20$ \\
\hline & & 17.42 & $9.52 \mathrm{E}+03$ & 611.41 & 157.12 & $6.49 \mathrm{E}+14$ & $16.91 \%$ & $1.06 \mathrm{E}+21$ \\
\hline & & 16.18 & $1.08 \mathrm{E}+04$ & 695.60 & 178.13 & $7.17 \mathrm{E}+14$ & $19.24 \%$ & $1.22 \mathrm{E}+21$ \\
\hline \multirow{5}{*}{ A-2 } & \multirow{5}{*}{ L1B33Z } & 13.89 & $1.29 \mathrm{E}+04$ & 776.51 & 213.67 & $8.20 \mathrm{E}+14$ & $21.39 \%$ & $1.47 \mathrm{E}+21$ \\
\hline & & 12.65 & $1.34 \mathrm{E}+04$ & 804.42 & 221.16 & $8.66 \mathrm{E}+14$ & $21.93 \%$ & $1.51 \mathrm{E}+21$ \\
\hline & & 11.41 & $1.41 \mathrm{E}+04$ & 849.89 & 233.24 & $9.11 \mathrm{E}+14$ & $23.14 \%$ & $1.61 \mathrm{E}+21$ \\
\hline & & 10.17 & $1.46 \mathrm{E}+04$ & 880.02 & 241.16 & $9.44 \mathrm{E}+14$ & $24.10 \%$ & $1.68 \mathrm{E}+21$ \\
\hline & & 8.93 & $1.57 \mathrm{E}+04$ & 947.19 & 258.85 & $9.76 \mathrm{E}+14$ & $25.90 \%$ & $1.82 \mathrm{E}+21$ \\
\hline \multirow{5}{*}{ A-3 } & \multirow{5}{*}{ L1B32Z } & 6.63 & $1.43 \mathrm{E}+04$ & 1018.05 & 235.99 & $1.01 \mathrm{E}+15$ & $27.98 \%$ & $1.68 \mathrm{E}+21$ \\
\hline & & 5.39 & $1.42 \mathrm{E}+04$ & 1008.11 & 233.85 & $1.03 \mathrm{E}+15$ & $27.49 \%$ & $1.65 \mathrm{E}+21$ \\
\hline & & 4.15 & $1.43 \mathrm{E}+04$ & 1015.36 & 235.41 & $1.03 \mathrm{E}+15$ & $27.89 \%$ & $1.68 \mathrm{E}+21$ \\
\hline & & 2.91 & $1.46 \mathrm{E}+04$ & 1039.46 & 240.85 & $1.05 \mathrm{E}+15$ & $28.26 \%$ & $1.70 \mathrm{E}+21$ \\
\hline & & 1.68 & $1.51 \mathrm{E}+04$ & 1079.59 & 249.71 & $1.05 \mathrm{E}+15$ & $29.41 \%$ & $1.78 \mathrm{E}+21$ \\
\hline \multirow{5}{*}{ A-4 } & \multirow{5}{*}{ L1B51Z } & -1.68 & $1.67 \mathrm{E}+04$ & 1046.72 & 275.59 & $1.06 \mathrm{E}+15$ & $28.46 \%$ & $1.95 \mathrm{E}+21$ \\
\hline & & -2.91 & $1.61 \mathrm{E}+04$ & 1009.62 & 266.29 & $1.06 \mathrm{E}+15$ & $27.35 \%$ & $1.87 \mathrm{E}+21$ \\
\hline & & -4.15 & $1.61 \mathrm{E}+04$ & 1009.43 & 266.22 & $1.06 \mathrm{E}+15$ & $27.35 \%$ & $1.87 \mathrm{E}+21$ \\
\hline & & -5.39 & $1.57 \mathrm{E}+04$ & 983.06 & 259.44 & $1.04 \mathrm{E}+15$ & $27.00 \%$ & $1.84 \mathrm{E}+21$ \\
\hline & & -6.63 & $1.60 \mathrm{E}+04$ & 1004.36 & 264.81 & $1.04 \mathrm{E}+15$ & $27.63 \%$ & $1.89 \mathrm{E}+21$ \\
\hline \multirow{5}{*}{ A-5 } & \multirow{5}{*}{ L1B52Z } & -8.93 & $1.60 \mathrm{E}+04$ & 980.67 & 264.62 & $1.01 \mathrm{E}+15$ & $26.89 \%$ & $1.87 \mathrm{E}+21$ \\
\hline & & -10.17 & $1.51 \mathrm{E}+04$ & 921.71 & 249.30 & $9.78 \mathrm{E}+14$ & $25.25 \%$ & $1.74 \mathrm{E}+21$ \\
\hline & & -11.41 & $1.46 \mathrm{E}+04$ & 891.53 & 241.44 & $9.52 \mathrm{E}+14$ & $24.44 \%$ & $1.68 \mathrm{E}+21$ \\
\hline & & -12.65 & $1.41 \mathrm{E}+04$ & 858.54 & 232.87 & $9.19 \mathrm{E}+14$ & $23.49 \%$ & $1.61 \mathrm{E}+21$ \\
\hline & & -13.89 & $1.36 \mathrm{E}+04$ & 827.55 & 224.64 & $8.75 \mathrm{E}+14$ & $22.96 \%$ & $1.57 \mathrm{E}+21$ \\
\hline \multirow{5}{*}{ A-6 } & \multirow{5}{*}{ L1B53Z } & -16.18 & $1.23 \mathrm{E}+04$ & 753.81 & 202.66 & $7.89 \mathrm{E}+14$ & $20.84 \%$ & $1.40 \mathrm{E}+21$ \\
\hline & & -17.42 & $1.12 \mathrm{E}+04$ & 685.28 & 184.83 & $7.35 \mathrm{E}+14$ & $18.62 \%$ & $1.25 \mathrm{E}+21$ \\
\hline & & -18.66 & $1.02 \mathrm{E}+04$ & 621.10 & 167.97 & $6.67 \mathrm{E}+14$ & $16.95 \%$ & $1.13 \mathrm{E}+21$ \\
\hline & & -19.90 & $9.19 \mathrm{E}+03$ & 559.98 & 151.77 & $5.97 \mathrm{E}+14$ & $15.44 \%$ & $1.02 \mathrm{E}+21$ \\
\hline & & -21.14 & $8.06 \mathrm{E}+03$ & 489.51 & 133.06 & $5.11 \mathrm{E}+14$ & $13.51 \%$ & $8.87 \mathrm{E}+20$ \\
\hline
\end{tabular}

(a) Elevations for MCNP cells are reported at cell centroids relative to the modeled core axial midplane at 24.0 inches. 
Table 13: Cycle 144B, MCNP-Calculated HGR, Neutron Flux, Depletion, and Fission Density for AFIP-4 Fuel Foils in Position B, 38 EFPD (MOC2), Center Lobe Power at 22.4 MW (38 EFPD Cumulative). ${ }^{3}$

\begin{tabular}{|c|c|c|c|c|c|c|c|c|}
\hline $\begin{array}{c}\text { Plate } \\
\text { ID }\end{array}$ & $\begin{array}{l}\text { Fuel } \\
\text { Plate }\end{array}$ & $\begin{array}{c}\text { MCNP } \\
\text { Elevation }^{\mathrm{a}} \\
\text { (inches) }^{2}\end{array}$ & $\begin{array}{l}\text { Fission } \\
\text { Power } \\
\text { Density } \\
\text { (W/cc) } \\
\end{array}$ & $\begin{array}{c}\text { Fission } \\
\text { Heat } \\
\text { Rate } \\
(W / g) \\
\end{array}$ & $\begin{array}{c}\text { Surface } \\
\text { Heat } \\
\text { Flux } \\
(\mathbf{W} / \mathbf{c m} 2) \\
\end{array}$ & $\begin{array}{c}\text { Neutron } \\
\text { Flux } \\
\text { (n/cm2sec) } \\
\end{array}$ & $\begin{array}{c}\% \\
\text { Depletion } \\
\text { U-235 } \\
(\%) \\
\end{array}$ & $\begin{array}{c}\text { Fission } \\
\text { Density } \\
\text { (fissions/cc) }\end{array}$ \\
\hline \multirow{5}{*}{ B-1 } & \multirow{5}{*}{ L1H34Z } & 21.14 & $6.42 \mathrm{E}+03$ & 402.82 & 106.05 & $4.26 \mathrm{E}+14$ & $11.29 \%$ & $7.13 \mathrm{E}+20$ \\
\hline & & 19.90 & $7.43 \mathrm{E}+03$ & 467.10 & 122.64 & $5.05 \mathrm{E}+14$ & $13.11 \%$ & $8.31 \mathrm{E}+20$ \\
\hline & & 18.66 & $8.49 \mathrm{E}+03$ & 535.11 & 140.13 & $5.78 \mathrm{E}+14$ & $14.84 \%$ & $9.48 \mathrm{E}+20$ \\
\hline & & 17.42 & $9.50 \mathrm{E}+03$ & 600.78 & 156.92 & $6.49 \mathrm{E}+14$ & $16.58 \%$ & $1.06 \mathrm{E}+21$ \\
\hline & & 16.18 & $1.06 \mathrm{E}+04$ & 672.46 & 175.06 & $7.10 \mathrm{E}+14$ & $18.77 \%$ & $1.21 \mathrm{E}+21$ \\
\hline \multirow{5}{*}{ B-2 } & \multirow{5}{*}{ L1H35Z } & 13.89 & $1.21 \mathrm{E}+04$ & 769.05 & 199.88 & $8.11 \mathrm{E}+14$ & $21.25 \%$ & $1.39 \mathrm{E}+21$ \\
\hline & & 12.65 & $1.24 \mathrm{E}+04$ & 787.34 & 204.50 & $8.57 \mathrm{E}+14$ & $21.62 \%$ & $1.41 \mathrm{E}+21$ \\
\hline & & 11.41 & $1.30 \mathrm{E}+04$ & 827.57 & 214.55 & $8.94 \mathrm{E}+14$ & $22.83 \%$ & $1.50 \mathrm{E}+21$ \\
\hline & & 10.17 & $1.37 \mathrm{E}+04$ & 873.07 & 225.98 & $9.40 \mathrm{E}+14$ & $23.92 \%$ & $1.58 \mathrm{E}+21$ \\
\hline & & 8.93 & $1.45 \mathrm{E}+04$ & 926.83 & 239.26 & $9.63 \mathrm{E}+14$ & $25.64 \%$ & $1.70 \mathrm{E}+21$ \\
\hline \multirow{5}{*}{ B-3 } & \multirow{5}{*}{ L1H36Z } & 6.63 & $1.55 \mathrm{E}+04$ & 978.20 & 255.99 & $1.01 \mathrm{E}+15$ & $27.04 \%$ & $1.83 \mathrm{E}+21$ \\
\hline & & 5.39 & $1.52 \mathrm{E}+04$ & 959.74 & 251.38 & $1.02 \mathrm{E}+15$ & $26.41 \%$ & $1.78 \mathrm{E}+21$ \\
\hline & & 4.15 & $1.53 \mathrm{E}+04$ & 965.40 & 252.81 & $1.03 \mathrm{E}+15$ & $26.60 \%$ & $1.80 \mathrm{E}+21$ \\
\hline & & 2.91 & $1.55 \mathrm{E}+04$ & 977.19 & 255.75 & $1.04 \mathrm{E}+15$ & $26.91 \%$ & $1.82 \mathrm{E}+21$ \\
\hline & & 1.68 & $1.62 \mathrm{E}+04$ & 1023.30 & 267.22 & $1.05 \mathrm{E}+15$ & $28.38 \%$ & $1.93 \mathrm{E}+21$ \\
\hline \multirow{5}{*}{ B-4 } & \multirow{5}{*}{ L1H37Z } & -1.68 & $1.63 \mathrm{E}+04$ & 1018.57 & 268.75 & $1.05 \mathrm{E}+15$ & $28.07 \%$ & $1.93 \mathrm{E}+21$ \\
\hline & & -2.91 & $1.57 \mathrm{E}+04$ & 981.88 & 259.58 & $1.05 \mathrm{E}+15$ & $26.91 \%$ & $1.84 \mathrm{E}+21$ \\
\hline & & -4.15 & $1.57 \mathrm{E}+04$ & 982.28 & 259.71 & $1.04 \mathrm{E}+15$ & $26.75 \%$ & $1.83 \mathrm{E}+21$ \\
\hline & & -5.39 & $1.55 \mathrm{E}+04$ & 968.31 & 256.11 & $1.03 \mathrm{E}+15$ & $26.54 \%$ & $1.82 \mathrm{E}+21$ \\
\hline & & -6.63 & $1.58 \mathrm{E}+04$ & 984.51 & 260.15 & $1.03 \mathrm{E}+15$ & $27.22 \%$ & $1.87 \mathrm{E}+21$ \\
\hline \multirow{5}{*}{ B-5 } & \multirow{5}{*}{ L1H38Z } & -8.93 & $1.48 \mathrm{E}+04$ & 969.73 & 244.09 & $9.96 \mathrm{E}+14$ & $26.54 \%$ & $1.73 \mathrm{E}+21$ \\
\hline & & -10.17 & $1.38 \mathrm{E}+04$ & 906.08 & 228.62 & $9.76 \mathrm{E}+14$ & $24.93 \%$ & $1.61 \mathrm{E}+21$ \\
\hline & & -11.41 & $1.34 \mathrm{E}+04$ & 874.61 & 220.94 & $9.47 \mathrm{E}+14$ & $24.17 \%$ & $1.56 \mathrm{E}+21$ \\
\hline & & -12.65 & $1.28 \mathrm{E}+04$ & 834.91 & 211.26 & $9.04 \mathrm{E}+14$ & $23.16 \%$ & $1.48 \mathrm{E}+21$ \\
\hline & & -13.89 & $1.27 \mathrm{E}+04$ & 829.28 & 209.89 & $8.72 \mathrm{E}+14$ & $22.98 \%$ & $1.47 \mathrm{E}+21$ \\
\hline \multirow{5}{*}{ B-6 } & \multirow{5}{*}{ L1H39Z } & -16.18 & $1.19 \mathrm{E}+04$ & 741.14 & 195.81 & $7.85 \mathrm{E}+14$ & $20.52 \%$ & $1.36 \mathrm{E}+21$ \\
\hline & & -17.42 & $1.08 \mathrm{E}+04$ & 671.36 & 177.96 & $7.23 \mathrm{E}+14$ & $18.32 \%$ & $1.20 \mathrm{E}+21$ \\
\hline & & -18.66 & $9.74 \mathrm{E}+03$ & 604.94 & 160.75 & $6.62 \mathrm{E}+14$ & $16.69 \%$ & $1.09 \mathrm{E}+21$ \\
\hline & & -19.90 & $8.79 \mathrm{E}+03$ & 544.94 & 145.12 & $5.95 \mathrm{E}+14$ & $15.23 \%$ & $9.91 \mathrm{E}+20$ \\
\hline & & -21.14 & $7.80 \mathrm{E}+03$ & 482.36 & 128.82 & $5.08 \mathrm{E}+14$ & $13.28 \%$ & $8.62 \mathrm{E}+20$ \\
\hline
\end{tabular}

(a) Elevations for MCNP cells are reported at cell centroids relative to the modeled core axial midplane at 24.0 inches. 
Table 14: Cycle 144B, MCNP-Calculated HGR, Neutron Flux, Depletion, and Fission Density for AFIP-4 Fuel Foils in Position A, 51.7 EFPD (EOC), Center Lobe Power at 22.4MW (51.7 EFPD Cumulative). ${ }^{3}$

\begin{tabular}{|c|c|c|c|c|c|c|c|c|}
\hline $\begin{array}{c}\text { Plate } \\
\text { ID }\end{array}$ & $\begin{array}{l}\text { Fuel } \\
\text { Plate } \\
\end{array}$ & $\begin{array}{c}\text { MCNP } \\
\text { Elevation }^{\mathrm{a}} \\
\text { (inches) }^{-}\end{array}$ & $\begin{array}{l}\text { Fission } \\
\text { Power } \\
\text { Density } \\
\text { (W/cc) } \\
\end{array}$ & $\begin{array}{c}\text { Fission } \\
\text { Heat } \\
\text { Rate } \\
(W / g) \\
\end{array}$ & $\begin{array}{c}\text { Surface } \\
\text { Heat } \\
\text { Flux } \\
(\mathbf{W} / \mathbf{c m} 2) \\
\end{array}$ & $\begin{array}{c}\begin{array}{c}\text { Neutron } \\
\text { Flux } \\
\text { (n/cm2sec) }\end{array} \\
\end{array}$ & $\begin{array}{c}\% \\
\text { Depletion } \\
\text { U-235 } \\
(\%) \\
\end{array}$ & $\begin{array}{c}\text { Fission } \\
\text { Density } \\
\text { (fissions/cc) }\end{array}$ \\
\hline \multirow{5}{*}{ A-1 } & \multirow{5}{*}{ L1B31Z } & 21.14 & $6.42 \mathrm{E}+03$ & 411.64 & 105.97 & $4.32 \mathrm{E}+14$ & $15.67 \%$ & $9.76 \mathrm{E}+20$ \\
\hline & & 19.90 & $7.39 \mathrm{E}+03$ & 475.63 & 121.97 & $5.07 \mathrm{E}+14$ & $18.24 \%$ & $1.14 \mathrm{E}+21$ \\
\hline & & 18.66 & $8.38 \mathrm{E}+03$ & 541.20 & 138.30 & $5.80 \mathrm{E}+14$ & $20.59 \%$ & $1.29 \mathrm{E}+21$ \\
\hline & & 17.42 & $9.17 \mathrm{E}+03$ & 594.28 & 151.36 & $6.49 \mathrm{E}+14$ & $22.76 \%$ & $1.44 \mathrm{E}+21$ \\
\hline & & 16.18 & $1.03 \mathrm{E}+04$ & 672.34 & 170.44 & $7.16 \mathrm{E}+14$ & $25.80 \%$ & $1.65 \mathrm{E}+21$ \\
\hline \multirow{5}{*}{ A-2 } & \multirow{5}{*}{ L1B33Z } & 13.89 & $1.23 \mathrm{E}+04$ & 745.49 & 202.84 & $8.18 \mathrm{E}+14$ & $28.61 \%$ & $1.98 \mathrm{E}+21$ \\
\hline & & 12.65 & $1.27 \mathrm{E}+04$ & 771.14 & 209.57 & $8.66 \mathrm{E}+14$ & $29.36 \%$ & $2.04 \mathrm{E}+21$ \\
\hline & & 11.41 & $1.34 \mathrm{E}+04$ & 814.40 & 220.76 & $9.10 \mathrm{E}+14$ & $30.94 \%$ & $2.16 \mathrm{E}+21$ \\
\hline & & 10.17 & $1.38 \mathrm{E}+04$ & 840.64 & 227.47 & $9.43 \mathrm{E}+14$ & $32.14 \%$ & $2.25 \mathrm{E}+21$ \\
\hline & & 8.93 & $1.46 \mathrm{E}+04$ & 896.34 & 241.64 & $9.74 \mathrm{E}+14$ & $34.47 \%$ & $2.43 \mathrm{E}+21$ \\
\hline \multirow{5}{*}{ A-3 } & \multirow{5}{*}{ L1B32Z } & 6.63 & $1.32 \mathrm{E}+04$ & 956.21 & 218.46 & $1.01 \mathrm{E}+15$ & $37.05 \%$ & $2.25 \mathrm{E}+21$ \\
\hline & & 5.39 & $1.32 \mathrm{E}+04$ & 951.67 & 217.58 & $1.02 \mathrm{E}+15$ & $36.51 \%$ & $2.21 \mathrm{E}+21$ \\
\hline & & 4.15 & $1.32 \mathrm{E}+04$ & 953.78 & 217.94 & $1.03 \mathrm{E}+15$ & $36.96 \%$ & $2.24 \mathrm{E}+21$ \\
\hline & & 2.91 & $1.35 \mathrm{E}+04$ & 976.66 & 222.96 & $1.05 \mathrm{E}+15$ & $37.48 \%$ & $2.28 \mathrm{E}+21$ \\
\hline & & 1.68 & $1.39 \mathrm{E}+04$ & 1008.94 & 229.81 & $1.05 \mathrm{E}+15$ & $38.94 \%$ & $2.38 \mathrm{E}+21$ \\
\hline \multirow{5}{*}{ A-4 } & \multirow{5}{*}{ L1B51Z } & -1.68 & $1.54 \mathrm{E}+04$ & 981.20 & 254.53 & $1.05 \mathrm{E}+15$ & $37.77 \%$ & $2.61 \mathrm{E}+21$ \\
\hline & & -2.91 & $1.50 \mathrm{E}+04$ & 954.47 & 248.12 & $1.06 \mathrm{E}+15$ & $36.38 \%$ & $2.50 \mathrm{E}+21$ \\
\hline & & -4.15 & $1.50 \mathrm{E}+04$ & 952.72 & 247.68 & $1.05 \mathrm{E}+15$ & $36.38 \%$ & $2.50 \mathrm{E}+21$ \\
\hline & & -5.39 & $1.47 \mathrm{E}+04$ & 931.50 & 242.40 & $1.04 \mathrm{E}+15$ & $35.84 \%$ & $2.46 \mathrm{E}+21$ \\
\hline & & -6.63 & $1.49 \mathrm{E}+04$ & 946.57 & 245.99 & $1.03 \mathrm{E}+15$ & $36.63 \%$ & $2.52 \mathrm{E}+21$ \\
\hline \multirow{5}{*}{ A-5 } & \multirow{5}{*}{ L1B52Z } & -8.93 & $1.49 \mathrm{E}+04$ & 925.09 & 246.13 & $1.00 \mathrm{E}+15$ & $35.72 \%$ & $2.50 \mathrm{E}+21$ \\
\hline & & -10.17 & $1.42 \mathrm{E}+04$ & 878.34 & 234.47 & $9.76 \mathrm{E}+14$ & $33.63 \%$ & $2.34 \mathrm{E}+21$ \\
\hline & & -11.41 & $1.38 \mathrm{E}+04$ & 852.31 & 227.90 & $9.51 \mathrm{E}+14$ & $32.57 \%$ & $2.26 \mathrm{E}+21$ \\
\hline & & -12.65 & $1.33 \mathrm{E}+04$ & 819.60 & 219.58 & $9.17 \mathrm{E}+14$ & $31.37 \%$ & $2.17 \mathrm{E}+21$ \\
\hline & & -13.89 & $1.29 \mathrm{E}+04$ & 791.87 & 212.39 & $8.73 \mathrm{E}+14$ & $30.60 \%$ & $2.11 \mathrm{E}+21$ \\
\hline \multirow{5}{*}{ A-6 } & \multirow{5}{*}{ L1B53Z } & -16.18 & $1.17 \mathrm{E}+04$ & 725.97 & 193.04 & $7.89 \mathrm{E}+14$ & $27.89 \%$ & $1.89 \mathrm{E}+21$ \\
\hline & & -17.42 & $1.07 \mathrm{E}+04$ & 663.61 & 177.21 & $7.34 \mathrm{E}+14$ & $25.11 \%$ & $1.69 \mathrm{E}+21$ \\
\hline & & -18.66 & $9.79 \mathrm{E}+03$ & 603.35 & 161.68 & $6.67 \mathrm{E}+14$ & $22.91 \%$ & $1.53 \mathrm{E}+21$ \\
\hline & & -19.90 & $8.91 \mathrm{E}+03$ & 547.09 & 147.07 & $5.98 \mathrm{E}+14$ & $20.87 \%$ & $1.38 \mathrm{E}+21$ \\
\hline & & -21.14 & $7.85 \mathrm{E}+03$ & 480.29 & 129.60 & $5.13 \mathrm{E}+14$ & $18.30 \%$ & $1.20 \mathrm{E}+21$ \\
\hline
\end{tabular}

(a) Elevations for MCNP cells are reported at cell centroids relative to the modeled core axial midplane at 24.0 inches. 
Table 15: Cycle 144B, MCNP-Calculated HGR, Neutron Flux, Depletion, and Fission Density for AFIP-4 Fuel Foils in Position B, 51.7 EFPD (EOC), Center Lobe Power at 22.4 MW (51.7 EFPD Cumulative). ${ }^{3}$

\begin{tabular}{|c|c|c|c|c|c|c|c|c|}
\hline $\begin{array}{c}\text { Plate } \\
\text { ID }\end{array}$ & $\begin{array}{l}\text { Fuel } \\
\text { Plate } \\
\end{array}$ & $\begin{array}{c}\text { MCNP } \\
\text { Elevation }^{a} \\
\text { (inches) }\end{array}$ & $\begin{array}{l}\text { Fission } \\
\text { Power } \\
\text { Density } \\
(\mathrm{W} / \mathrm{cc}) \\
\end{array}$ & $\begin{array}{c}\text { Fission } \\
\text { Heat } \\
\text { Rate } \\
(\mathbf{W} / \mathrm{g}) \\
\end{array}$ & $\begin{array}{c}\text { Surface } \\
\text { Heat } \\
\text { Flux } \\
(\mathbf{W} / \mathbf{c m} 2) \\
\end{array}$ & $\begin{array}{c}\begin{array}{c}\text { Neutron } \\
\text { Flux } \\
(\mathrm{n} / \mathrm{cm} 2 \mathrm{sec}) \\
\end{array} \\
\end{array}$ & $\begin{array}{c}\% \\
\text { Depletion } \\
\text { U-235 (\%) } \\
\end{array}$ & $\begin{array}{c}\text { Fission } \\
\text { Density } \\
\text { (fissions/cc) }\end{array}$ \\
\hline \multirow{5}{*}{ B-1 } & \multirow{5}{*}{ L1H34Z } & 21.14 & $6.28 \mathrm{E}+03$ & 396.49 & 103.74 & $4.27 \mathrm{E}+14$ & $15.33 \%$ & $9.67 \mathrm{E}+20$ \\
\hline & & 19.90 & $7.22 \mathrm{E}+03$ & 457.38 & 119.27 & $5.06 \mathrm{E}+14$ & $17.69 \%$ & $1.12 \mathrm{E}+21$ \\
\hline & & 18.66 & $8.22 \mathrm{E}+03$ & 522.10 & 135.65 & $5.77 \mathrm{E}+14$ & $20.03 \%$ & $1.28 \mathrm{E}+21$ \\
\hline & & 17.42 & $9.16 \mathrm{E}+03$ & 584.24 & 151.26 & $6.49 \mathrm{E}+14$ & $22.34 \%$ & $1.44 \mathrm{E}+21$ \\
\hline & & 16.18 & $1.01 \mathrm{E}+04$ & 649.97 & 167.58 & $7.10 \mathrm{E}+14$ & $25.13 \%$ & $1.63 \mathrm{E}+21$ \\
\hline \multirow{5}{*}{ B-2 } & \multirow{5}{*}{ L1H35Z } & 13.89 & $1.15 \mathrm{E}+04$ & 739.58 & 190.07 & $8.11 \mathrm{E}+14$ & $28.42 \%$ & $1.86 \mathrm{E}+21$ \\
\hline & & 12.65 & $1.18 \mathrm{E}+04$ & 757.91 & 194.62 & $8.56 \mathrm{E}+14$ & $28.93 \%$ & $1.90 \mathrm{E}+21$ \\
\hline & & 11.41 & $1.23 \mathrm{E}+04$ & 794.01 & 203.41 & $8.92 \mathrm{E}+14$ & $30.47 \%$ & $2.01 \mathrm{E}+21$ \\
\hline & & 10.17 & $1.29 \mathrm{E}+04$ & 835.02 & 213.43 & $9.39 \mathrm{E}+14$ & $31.91 \%$ & $2.12 \mathrm{E}+21$ \\
\hline & & 8.93 & $1.36 \mathrm{E}+04$ & 879.73 & 224.10 & $9.62 \mathrm{E}+14$ & $34.06 \%$ & $2.27 \mathrm{E}+21$ \\
\hline \multirow{5}{*}{ B-3 } & \multirow{5}{*}{ L1H36Z } & 6.63 & $1.45 \mathrm{E}+04$ & 924.78 & 238.63 & $1.01 \mathrm{E}+15$ & $35.87 \%$ & $2.44 \mathrm{E}+21$ \\
\hline & & 5.39 & $1.43 \mathrm{E}+04$ & 911.41 & 235.48 & $1.02 \mathrm{E}+15$ & $35.07 \%$ & $2.38 \mathrm{E}+21$ \\
\hline & & 4.15 & $1.43 \mathrm{E}+04$ & 916.58 & 236.70 & $1.03 \mathrm{E}+15$ & $35.32 \%$ & $2.40 \mathrm{E}+21$ \\
\hline & & 2.91 & $1.44 \mathrm{E}+04$ & 924.17 & 238.55 & $1.04 \mathrm{E}+15$ & $35.71 \%$ & $2.43 \mathrm{E}+21$ \\
\hline & & 1.68 & $1.50 \mathrm{E}+04$ & 960.29 & 247.16 & $1.04 \mathrm{E}+15$ & $37.53 \%$ & $2.57 \mathrm{E}+21$ \\
\hline \multirow{5}{*}{ B-4 } & \multirow{5}{*}{ L1H37Z } & -1.68 & $1.51 \mathrm{E}+04$ & 960.68 & 249.85 & $1.05 \mathrm{E}+15$ & $37.14 \%$ & $2.58 \mathrm{E}+21$ \\
\hline & & -2.91 & $1.47 \mathrm{E}+04$ & 928.28 & 241.98 & $1.05 \mathrm{E}+15$ & $35.74 \%$ & $2.46 \mathrm{E}+21$ \\
\hline & & -4.15 & $1.47 \mathrm{E}+04$ & 930.30 & 242.56 & $1.04 \mathrm{E}+15$ & $35.58 \%$ & $2.45 \mathrm{E}+21$ \\
\hline & & -5.39 & $1.45 \mathrm{E}+04$ & 920.68 & 240.15 & $1.03 \mathrm{E}+15$ & $35.23 \%$ & $2.43 \mathrm{E}+21$ \\
\hline & & -6.63 & $1.47 \mathrm{E}+04$ & 930.99 & 242.57 & $1.03 \mathrm{E}+15$ & $36.05 \%$ & $2.49 \mathrm{E}+21$ \\
\hline \multirow{5}{*}{ B-5 } & \multirow{5}{*}{ L1H38Z } & -8.93 & $1.38 \mathrm{E}+04$ & 916.40 & 227.50 & $9.96 \mathrm{E}+14$ & $35.28 \%$ & $2.31 \mathrm{E}+21$ \\
\hline & & -10.17 & $1.30 \mathrm{E}+04$ & 864.16 & 215.25 & $9.75 \mathrm{E}+14$ & $33.20 \%$ & $2.16 \mathrm{E}+21$ \\
\hline & & -11.41 & $1.26 \mathrm{E}+04$ & 835.19 & 208.36 & $9.47 \mathrm{E}+14$ & $32.17 \%$ & $2.08 \mathrm{E}+21$ \\
\hline & & -12.65 & $1.21 \mathrm{E}+04$ & 801.40 & 200.35 & $9.02 \mathrm{E}+14$ & $30.84 \%$ & $1.99 \mathrm{E}+21$ \\
\hline & & -13.89 & $1.21 \mathrm{E}+04$ & 795.67 & 198.99 & $8.73 \mathrm{E}+14$ & $30.64 \%$ & $1.98 \mathrm{E}+21$ \\
\hline \multirow{5}{*}{ B-6 } & \multirow{5}{*}{ L1H39Z } & -16.18 & $1.13 \mathrm{E}+04$ & 714.78 & 186.81 & $7.85 \mathrm{E}+14$ & $27.44 \%$ & $1.83 \mathrm{E}+21$ \\
\hline & & -17.42 & $1.04 \mathrm{E}+04$ & 652.35 & 171.25 & $7.22 \mathrm{E}+14$ & $24.65 \%$ & $1.63 \mathrm{E}+21$ \\
\hline & & -18.66 & $9.39 \mathrm{E}+03$ & 588.71 & 155.07 & $6.63 \mathrm{E}+14$ & $22.47 \%$ & $1.48 \mathrm{E}+21$ \\
\hline & & -19.90 & $8.53 \mathrm{E}+03$ & 532.76 & 140.76 & $5.95 \mathrm{E}+14$ & $20.50 \%$ & $1.34 \mathrm{E}+21$ \\
\hline & & -21.14 & $7.59 \mathrm{E}+03$ & 472.39 & 125.28 & $5.09 \mathrm{E}+14$ & $17.99 \%$ & $1.17 \mathrm{E}+21$ \\
\hline
\end{tabular}

(a) Elevations for MCNP cells are reported at cell centroids relative to the modeled core axial midplane at 24.0 inches. 
Table 16: Cycle 145A, MCNP-Calculated HGR, Neutron Flux, Depletion, and Fission Density for AFIP-4 Fuel Foils in Position A, 0 EFPD (BOC), Center Lobe Power at 23.2 MW (51.7 EFPD Cumulative). ${ }^{3}$

\begin{tabular}{|c|c|c|c|c|c|c|}
\hline $\begin{array}{c}\text { Plate } \\
\text { ID }\end{array}$ & $\begin{array}{l}\text { Fuel } \\
\text { Plate } \\
\end{array}$ & $\begin{array}{c}\text { MCNP } \\
\text { Elevation }^{a} \\
\text { (inches) }^{a}\end{array}$ & $\begin{array}{c}\text { Fission } \\
\text { Power } \\
\text { Density } \\
\text { (W/cc) } \\
\end{array}$ & $\begin{array}{c}\text { Fission } \\
\text { Heat } \\
\text { Rate } \\
(\mathbf{W} / \mathrm{g}) \\
\end{array}$ & $\begin{array}{c}\text { Surface } \\
\text { Heat } \\
\text { Flux } \\
(\mathbf{W} / \mathbf{c m} 2) \\
\end{array}$ & $\begin{array}{c}\text { Neutron } \\
\text { Flux } \\
(\mathbf{n} / \mathbf{c m} 2 \mathrm{sec}) \\
\end{array}$ \\
\hline \multirow{5}{*}{ A-1 } & \multirow{5}{*}{ L1B31Z } & 21.14 & $5.76 \mathrm{E}+03$ & 366.73 & 95.15 & $3.99 \mathrm{E}+14$ \\
\hline & & 19.90 & $6.63 \mathrm{E}+03$ & 423.79 & 109.52 & $4.79 \mathrm{E}+14$ \\
\hline & & 18.66 & $7.54 \mathrm{E}+03$ & 483.57 & 124.53 & $5.52 \mathrm{E}+14$ \\
\hline & & 17.42 & $8.39 \mathrm{E}+03$ & 539.33 & 138.44 & $6.21 \mathrm{E}+14$ \\
\hline & & 16.18 & $9.54 \mathrm{E}+03$ & 616.57 & 157.52 & $6.91 \mathrm{E}+14$ \\
\hline \multirow{5}{*}{ A-2 } & \multirow{5}{*}{ L1B33Z } & 13.89 & $1.16 \mathrm{E}+04$ & 696.77 & 191.06 & $8.03 \mathrm{E}+14$ \\
\hline & & 12.65 & $1.18 \mathrm{E}+04$ & 712.01 & 195.01 & $8.53 \mathrm{E}+14$ \\
\hline & & 11.41 & $1.23 \mathrm{E}+04$ & 745.41 & 203.64 & $9.03 \mathrm{E}+14$ \\
\hline & & 10.17 & $1.29 \mathrm{E}+04$ & 782.91 & 213.50 & $9.43 \mathrm{E}+14$ \\
\hline & & 8.93 & $1.37 \mathrm{E}+04$ & 835.33 & 226.95 & $9.79 \mathrm{E}+14$ \\
\hline \multirow{5}{*}{ A-3 } & \multirow{5}{*}{ L1B32Z } & 6.63 & $1.27 \mathrm{E}+04$ & 907.93 & 209.05 & $1.02 \mathrm{E}+15$ \\
\hline & & 5.39 & $1.25 \mathrm{E}+04$ & 893.41 & 205.86 & $1.04 \mathrm{E}+15$ \\
\hline & & 4.15 & $1.26 \mathrm{E}+04$ & 905.36 & 208.49 & $1.06 \mathrm{E}+15$ \\
\hline & & 2.91 & $1.28 \mathrm{E}+04$ & 920.38 & 211.75 & $1.07 \mathrm{E}+15$ \\
\hline & & 1.68 & $1.32 \mathrm{E}+04$ & 946.26 & 217.21 & $1.08 \mathrm{E}+15$ \\
\hline \multirow{5}{*}{ A-4 } & \multirow{5}{*}{ L1B51Z } & -1.68 & $1.46 \mathrm{E}+04$ & 919.46 & 240.38 & $1.09 \mathrm{E}+15$ \\
\hline & & -2.91 & $1.43 \mathrm{E}+04$ & 902.29 & 236.38 & $1.08 \mathrm{E}+15$ \\
\hline & & -4.15 & $1.42 \mathrm{E}+04$ & 895.18 & 234.54 & $1.07 \mathrm{E}+15$ \\
\hline & & -5.39 & $1.43 \mathrm{E}+04$ & 898.02 & 235.48 & $1.06 \mathrm{E}+15$ \\
\hline & & -6.63 & $1.43 \mathrm{E}+04$ & 900.08 & 235.73 & $1.05 \mathrm{E}+15$ \\
\hline \multirow{5}{*}{ A-5 } & \multirow{5}{*}{ L1B52Z } & -8.93 & $1.40 \mathrm{E}+04$ & 864.88 & 231.91 & $1.02 \mathrm{E}+15$ \\
\hline & & -10.17 & $1.34 \mathrm{E}+04$ & 824.25 & 221.75 & $9.84 \mathrm{E}+14$ \\
\hline & & -11.41 & $1.30 \mathrm{E}+04$ & 795.92 & 214.49 & $9.57 \mathrm{E}+14$ \\
\hline & & -12.65 & $1.25 \mathrm{E}+04$ & 762.17 & 205.79 & $9.17 \mathrm{E}+14$ \\
\hline & & -13.89 & $1.23 \mathrm{E}+04$ & 748.79 & 202.40 & $8.73 \mathrm{E}+14$ \\
\hline \multirow{5}{*}{ A-6 } & \multirow{5}{*}{ L1B53Z } & -16.18 & $1.09 \mathrm{E}+04$ & 674.21 & 180.67 & $7.71 \mathrm{E}+14$ \\
\hline & & -17.42 & $9.86 \mathrm{E}+03$ & 604.82 & 162.77 & $7.12 \mathrm{E}+14$ \\
\hline & & -18.66 & $8.98 \mathrm{E}+03$ & 549.01 & 148.27 & $6.42 \mathrm{E}+14$ \\
\hline & & -19.90 & $8.12 \mathrm{E}+03$ & 494.74 & 134.03 & $5.69 \mathrm{E}+14$ \\
\hline & & -21.14 & $7.31 \mathrm{E}+03$ & 443.85 & 120.70 & $4.93 \mathrm{E}+14$ \\
\hline
\end{tabular}

(a) Elevations for MCNP cells are reported at cell centroids relative to the modeled core axial midplane at 24.0 inches. 
Table 17: Cycle 145A, MCNP-Calculated HGR, Neutron Flux, Depletion, and Fission Density for AFIP-4 Fuel Foils in Position B, 0 EFPD (BOC), Center Lobe Power at 23.2 MW (51.7 EFPD Cumulative). ${ }^{3}$

\begin{tabular}{|c|c|c|c|c|c|c|}
\hline $\begin{array}{c}\text { Plate } \\
\text { ID }\end{array}$ & $\begin{array}{l}\text { Fuel } \\
\text { Plate }\end{array}$ & $\begin{array}{c}\text { MCNP } \\
\text { Elevation }^{\mathrm{a}} \\
\text { (inches) }^{-}\end{array}$ & $\begin{array}{l}\text { Fission } \\
\text { Power } \\
\text { Density } \\
\text { (W/cc) } \\
\end{array}$ & $\begin{array}{c}\text { Fission } \\
\text { Heat } \\
\text { Rate } \\
(\mathbf{W} / \mathrm{g}) \\
\end{array}$ & $\begin{array}{c}\text { Surface } \\
\text { Heat } \\
\text { Flux } \\
(\mathbf{W} / \mathbf{c m} 2) \\
\end{array}$ & $\begin{array}{c}\text { Neutron } \\
\text { Flux } \\
\text { (n/cm2sec) }\end{array}$ \\
\hline \multirow{5}{*}{ B-1 } & \multirow{5}{*}{ L1H34Z } & 21.14 & $5.77 \mathrm{E}+03$ & 359.73 & 95.26 & $3.97 \mathrm{E}+14$ \\
\hline & & 19.90 & $6.66 \mathrm{E}+03$ & 416.55 & 109.93 & $4.77 \mathrm{E}+14$ \\
\hline & & 18.66 & $7.46 \mathrm{E}+03$ & 468.33 & 123.15 & $5.46 \mathrm{E}+14$ \\
\hline & & 17.42 & $8.39 \mathrm{E}+03$ & 528.89 & 138.59 & $6.19 \mathrm{E}+14$ \\
\hline & & 16.18 & $9.52 \mathrm{E}+03$ & 602.27 & 157.16 & $6.88 \mathrm{E}+14$ \\
\hline \multirow{5}{*}{ B-2 } & \multirow{5}{*}{ L1H35Z } & 13.89 & $1.08 \mathrm{E}+04$ & 682.51 & 177.52 & $7.94 \mathrm{E}+14$ \\
\hline & & 12.65 & $1.11 \mathrm{E}+04$ & 705.42 & 183.34 & $8.49 \mathrm{E}+14$ \\
\hline & & 11.41 & $1.17 \mathrm{E}+04$ & 743.00 & 192.65 & $8.95 \mathrm{E}+14$ \\
\hline & & 10.17 & $1.21 \mathrm{E}+04$ & 770.13 & 199.22 & $9.33 \mathrm{E}+14$ \\
\hline & & 8.93 & $1.30 \mathrm{E}+04$ & 832.44 & 214.62 & $9.73 \mathrm{E}+14$ \\
\hline \multirow{5}{*}{ B-3 } & \multirow{5}{*}{ L1H36Z } & 6.63 & $1.38 \mathrm{E}+04$ & 874.13 & 228.29 & $1.02 \mathrm{E}+15$ \\
\hline & & 5.39 & $1.38 \mathrm{E}+04$ & 872.28 & 228.09 & $1.04 \mathrm{E}+15$ \\
\hline & & 4.15 & $1.38 \mathrm{E}+04$ & 874.83 & 228.65 & $1.05 \mathrm{E}+15$ \\
\hline & & 2.91 & $1.41 \mathrm{E}+04$ & 890.53 & 232.65 & $1.06 \mathrm{E}+15$ \\
\hline & & 1.68 & $1.44 \mathrm{E}+04$ & 911.03 & 237.29 & $1.08 \mathrm{E}+15$ \\
\hline \multirow{5}{*}{ B-4 } & \multirow{5}{*}{ L1H37Z } & -1.68 & $1.45 \mathrm{E}+04$ & 911.19 & 239.85 & $1.08 \mathrm{E}+15$ \\
\hline & & -2.91 & $1.41 \mathrm{E}+04$ & 884.51 & 233.36 & $1.07 \mathrm{E}+15$ \\
\hline & & -4.15 & $1.42 \mathrm{E}+04$ & 888.74 & 234.52 & $1.07 \mathrm{E}+15$ \\
\hline & & -5.39 & $1.41 \mathrm{E}+04$ & 884.47 & 233.49 & $1.06 \mathrm{E}+15$ \\
\hline & & -6.63 & $1.42 \mathrm{E}+04$ & 890.07 & 234.71 & $1.04 \mathrm{E}+15$ \\
\hline \multirow{5}{*}{ B-5 } & \multirow{5}{*}{ L1H38Z } & -8.93 & $1.31 \mathrm{E}+04$ & 861.28 & 216.41 & $1.01 \mathrm{E}+15$ \\
\hline & & -10.17 & $1.24 \mathrm{E}+04$ & 814.21 & 205.26 & $9.80 \mathrm{E}+14$ \\
\hline & & -11.41 & $1.22 \mathrm{E}+04$ & 798.65 & 201.66 & $9.46 \mathrm{E}+14$ \\
\hline & & -12.65 & $1.16 \mathrm{E}+04$ & 758.99 & 192.04 & $9.11 \mathrm{E}+14$ \\
\hline & & -13.89 & $1.13 \mathrm{E}+04$ & 740.07 & 187.32 & $8.68 \mathrm{E}+14$ \\
\hline \multirow{5}{*}{ B-6 } & \multirow{5}{*}{ L1H39Z } & -16.18 & $1.06 \mathrm{E}+04$ & 661.39 & 174.95 & $7.69 \mathrm{E}+14$ \\
\hline & & -17.42 & $9.64 \mathrm{E}+03$ & 599.04 & 159.16 & $7.05 \mathrm{E}+14$ \\
\hline & & -18.66 & $8.80 \mathrm{E}+03$ & 544.93 & 145.27 & $6.38 \mathrm{E}+14$ \\
\hline & & -19.90 & $7.91 \mathrm{E}+03$ & 488.49 & 130.60 & $5.66 \mathrm{E}+14$ \\
\hline & & -21.14 & $7.13 \mathrm{E}+03$ & 438.66 & 117.74 & $4.88 \mathrm{E}+14$ \\
\hline
\end{tabular}

(a) Elevations for MCNP cells are reported at cell centroids relative to the modeled core axial midplane at 24.0 inches. 
Table 18: Cycle 145A, MCNP-Calculated HGR, Neutron Flux, Depletion, and Fission Density for AFIP-4 Fuel Foils in Position A, 18.0 EFPD (MOC1), Center Lobe Power at 23.2 MW (69.7 EFPD Cumulative). ${ }^{3}$

\begin{tabular}{|c|c|c|c|c|c|c|c|c|}
\hline $\begin{array}{c}\text { Plate } \\
\text { ID }\end{array}$ & $\begin{array}{l}\text { Fuel } \\
\text { Plate } \\
\end{array}$ & $\begin{array}{c}\text { MCNP } \\
\text { Elevation }^{a} \\
\text { (inches) }^{a}\end{array}$ & $\begin{array}{l}\text { Fission } \\
\text { Power } \\
\text { Density } \\
\text { (W/cc) } \\
\end{array}$ & $\begin{array}{c}\text { Fission } \\
\text { Heat } \\
\text { Rate } \\
(W / g) \\
\end{array}$ & $\begin{array}{c}\text { Surface } \\
\text { Heat } \\
\text { Flux } \\
(\mathbf{W} / \mathbf{c m} 2) \\
\end{array}$ & $\begin{array}{c}\begin{array}{c}\text { Neutron } \\
\text { Flux } \\
\text { (n/cm2sec) }\end{array} \\
\end{array}$ & $\begin{array}{c}\% \\
\text { Depletion } \\
\text { U-235 } \\
(\%) \\
\end{array}$ & $\begin{array}{c}\text { Fission } \\
\text { Density } \\
\text { (fissions/cc) }\end{array}$ \\
\hline \multirow{5}{*}{ A-1 } & \multirow{5}{*}{ L1B31Z } & 21.14 & $6.38 \mathrm{E}+03$ & 409.13 & 105.40 & $4.52 \mathrm{E}+14$ & $20.38 \%$ & $1.27 \mathrm{E}+21$ \\
\hline & & 19.90 & $7.16 \mathrm{E}+03$ & 460.85 & 118.13 & $5.29 \mathrm{E}+14$ & $23.59 \%$ & $1.48 \mathrm{E}+21$ \\
\hline & & 18.66 & $8.07 \mathrm{E}+03$ & 522.52 & 133.31 & $6.10 \mathrm{E}+14$ & $26.59 \%$ & $1.68 \mathrm{E}+21$ \\
\hline & & 17.42 & $8.85 \mathrm{E}+03$ & 574.89 & 146.04 & $6.80 \mathrm{E}+14$ & $29.38 \%$ & $1.87 \mathrm{E}+21$ \\
\hline & & 16.18 & $9.80 \mathrm{E}+03$ & 640.94 & 161.83 & $7.52 \mathrm{E}+14$ & $33.23 \%$ & $2.14 \mathrm{E}+21$ \\
\hline \multirow{5}{*}{ A-2 } & \multirow{5}{*}{ L1B33Z } & 13.89 & $1.17 \mathrm{E}+04$ & 714.96 & 193.47 & $8.52 \mathrm{E}+14$ & $36.83 \%$ & $2.58 \mathrm{E}+21$ \\
\hline & & 12.65 & $1.19 \mathrm{E}+04$ & 726.17 & 196.23 & $9.00 \mathrm{E}+14$ & $37.72 \%$ & $2.65 \mathrm{E}+21$ \\
\hline & & 11.41 & $1.24 \mathrm{E}+04$ & 757.87 & 204.15 & $9.41 \mathrm{E}+14$ & $39.62 \%$ & $2.79 \mathrm{E}+21$ \\
\hline & & 10.17 & $1.27 \mathrm{E}+04$ & 781.31 & 209.95 & $9.72 \mathrm{E}+14$ & $41.17 \%$ & $2.92 \mathrm{E}+21$ \\
\hline & & 8.93 & $1.32 \mathrm{E}+04$ & 812.92 & 217.43 & $1.00 \mathrm{E}+15$ & $43.96 \%$ & $3.14 \mathrm{E}+21$ \\
\hline \multirow{5}{*}{ A-3 } & \multirow{5}{*}{ L1B32Z } & 6.63 & $1.18 \mathrm{E}+04$ & 862.62 & 195.29 & $1.03 \mathrm{E}+15$ & $47.18 \%$ & $2.90 \mathrm{E}+21$ \\
\hline & & 5.39 & $1.17 \mathrm{E}+04$ & 852.36 & 193.16 & $1.04 \mathrm{E}+15$ & $46.45 \%$ & $2.85 \mathrm{E}+21$ \\
\hline & & 4.15 & $1.18 \mathrm{E}+04$ & 861.14 & 194.98 & $1.05 \mathrm{E}+15$ & $47.06 \%$ & $2.89 \mathrm{E}+21$ \\
\hline & & 2.91 & $1.18 \mathrm{E}+04$ & 862.59 & 195.11 & $1.06 \mathrm{E}+15$ & $47.70 \%$ & $2.93 \mathrm{E}+21$ \\
\hline & & 1.68 & $1.21 \mathrm{E}+04$ & 884.45 & 199.47 & $1.07 \mathrm{E}+15$ & $49.34 \%$ & $3.05 \mathrm{E}+21$ \\
\hline \multirow{5}{*}{ A-4 } & \multirow{5}{*}{ L1B51Z } & -1.68 & $1.34 \mathrm{E}+04$ & 860.13 & 221.05 & $1.07 \mathrm{E}+15$ & $47.98 \%$ & $3.36 \mathrm{E}+21$ \\
\hline & & -2.91 & $1.33 \mathrm{E}+04$ & 850.49 & 219.12 & $1.07 \mathrm{E}+15$ & $46.47 \%$ & $3.24 \mathrm{E}+21$ \\
\hline & & -4.15 & $1.33 \mathrm{E}+04$ & 850.21 & 219.07 & $1.07 \mathrm{E}+15$ & $46.41 \%$ & $3.23 \mathrm{E}+21$ \\
\hline & & -5.39 & $1.32 \mathrm{E}+04$ & 846.11 & 218.21 & $1.05 \mathrm{E}+15$ & $45.92 \%$ & $3.19 \mathrm{E}+21$ \\
\hline & & -6.63 & $1.32 \mathrm{E}+04$ & 849.09 & 218.68 & $1.05 \mathrm{E}+15$ & $46.71 \%$ & $3.25 \mathrm{E}+21$ \\
\hline \multirow{5}{*}{ A-5 } & \multirow{5}{*}{ L1B52Z } & -8.93 & $1.33 \mathrm{E}+04$ & 833.33 & 219.85 & $1.03 \mathrm{E}+15$ & $45.49 \%$ & $3.22 \mathrm{E}+21$ \\
\hline & & -10.17 & $1.27 \mathrm{E}+04$ & 793.49 & 210.19 & $9.96 \mathrm{E}+14$ & $43.05 \%$ & $3.03 \mathrm{E}+21$ \\
\hline & & -11.41 & $1.26 \mathrm{E}+04$ & 782.96 & 207.87 & $9.76 \mathrm{E}+14$ & $41.72 \%$ & $2.92 \mathrm{E}+21$ \\
\hline & & -12.65 & $1.22 \mathrm{E}+04$ & 756.22 & 201.25 & $9.47 \mathrm{E}+14$ & $40.23 \%$ & $2.81 \mathrm{E}+21$ \\
\hline & & -13.89 & $1.22 \mathrm{E}+04$ & 757.15 & 201.80 & $9.11 \mathrm{E}+14$ & $39.33 \%$ & $2.74 \mathrm{E}+21$ \\
\hline \multirow{5}{*}{ A-6 } & \multirow{5}{*}{ L1B53Z } & -16.18 & $1.10 \mathrm{E}+04$ & 688.35 & 182.15 & $8.11 \mathrm{E}+14$ & $35.89 \%$ & $2.45 \mathrm{E}+21$ \\
\hline & & -17.42 & $9.98 \mathrm{E}+03$ & 619.61 & 164.85 & $7.52 \mathrm{E}+14$ & $32.40 \%$ & $2.19 \mathrm{E}+21$ \\
\hline & & -18.66 & $9.27 \mathrm{E}+03$ & 572.90 & 153.10 & $6.91 \mathrm{E}+14$ & $29.61 \%$ & $1.99 \mathrm{E}+21$ \\
\hline & & -19.90 & $8.45 \mathrm{E}+03$ & 520.16 & 139.59 & $6.17 \mathrm{E}+14$ & $27.00 \%$ & $1.80 \mathrm{E}+21$ \\
\hline & & -21.14 & $7.67 \mathrm{E}+03$ & 469.53 & 126.63 & $5.37 \mathrm{E}+14$ & $23.87 \%$ & $1.58 \mathrm{E}+21$ \\
\hline
\end{tabular}

(a) Elevations for MCNP cells are reported at cell centroids relative to the modeled core axial midplane at 24.0 inches. 
Table 19: Cycle 145A, MCNP-Calculated HGR, Neutron Flux, Depletion, and Fission Density for AFIP-4 Fuel Foils in Position B, 18.0 EFPD (MOC1), Center Lobe Power at 23.2 MW (69.7 EFPD Cumulative). ${ }^{3}$

\begin{tabular}{|c|c|c|c|c|c|c|c|c|}
\hline $\begin{array}{c}\text { Plate } \\
\text { ID } \\
\end{array}$ & $\begin{array}{l}\text { Fuel } \\
\text { Plate }\end{array}$ & $\begin{array}{c}\text { MCNP } \\
\text { Elevation }^{\text {a }} \\
\text { (inches) }^{\text {inches }}\end{array}$ & $\begin{array}{c}\text { Fission } \\
\text { Power } \\
\text { Density } \\
\text { (W/cc) } \\
\end{array}$ & $\begin{array}{c}\text { Fission } \\
\text { Heat } \\
\text { Rate } \\
(\mathrm{W} / \mathrm{g}) \\
\end{array}$ & $\begin{array}{c}\text { Surface } \\
\text { Heat } \\
\text { Flux } \\
(W / c m 2) \\
\end{array}$ & $\begin{array}{c}\text { Neutron } \\
\text { Flux } \\
\text { (n/cm2sec) } \\
\end{array}$ & $\begin{array}{c}\% \\
\text { Depletion } \\
\text { U-235 } \\
(\%) \\
\end{array}$ & $\begin{array}{c}\text { Fission } \\
\text { Density } \\
\text { (fissions/ce) }\end{array}$ \\
\hline \multirow{5}{*}{ B-1 } & \multirow{5}{*}{ L1H34Z } & 21.14 & $6.29 \mathrm{E}+03$ & 394.89 & 103.86 & $4.47 \mathrm{E}+14$ & $19.89 \%$ & $1.26 \mathrm{E}+21$ \\
\hline & & 19.90 & $7.11 \mathrm{E}+03$ & 448.40 & 117.40 & $5.26 \mathrm{E}+14$ & $22.94 \%$ & $1.47 \mathrm{E}+21$ \\
\hline & & 18.66 & $7.92 \mathrm{E}+03$ & 501.57 & 130.72 & $6.01 \mathrm{E}+14$ & $25.87 \%$ & $1.67 \mathrm{E}+21$ \\
\hline & & 17.42 & $8.89 \mathrm{E}+03$ & 565.62 & 146.72 & $6.76 \mathrm{E}+14$ & $28.83 \%$ & $1.87 \mathrm{E}+21$ \\
\hline & & 16.18 & $9.96 \mathrm{E}+03$ & 637.24 & 164.37 & $7.42 \mathrm{E}+14$ & $32.40 \%$ & $2.12 \mathrm{E}+21$ \\
\hline \multirow{5}{*}{ B-2 } & \multirow{5}{*}{ L1H35Z } & 13.89 & $1.10 \mathrm{E}+04$ & 706.51 & 181.40 & $8.51 \mathrm{E}+14$ & $36.51 \%$ & $2.42 \mathrm{E}+21$ \\
\hline & & 12.65 & $1.12 \mathrm{E}+04$ & 719.63 & 184.56 & $8.90 \mathrm{E}+14$ & $37.23 \%$ & $2.47 \mathrm{E}+21$ \\
\hline & & 11.41 & $1.16 \mathrm{E}+04$ & 746.59 & 190.87 & $9.33 \mathrm{E}+14$ & $39.14 \%$ & $2.61 \mathrm{E}+21$ \\
\hline & & 10.17 & $1.19 \mathrm{E}+04$ & 767.98 & 195.80 & $9.62 \mathrm{E}+14$ & $40.79 \%$ & $2.74 \mathrm{E}+21$ \\
\hline & & 8.93 & $1.25 \mathrm{E}+04$ & 811.03 & 205.87 & $9.94 \mathrm{E}+14$ & $43.55 \%$ & $2.94 \mathrm{E}+21$ \\
\hline \multirow{5}{*}{ B-3 } & \multirow{5}{*}{ L1H36Z } & 6.63 & $1.31 \mathrm{E}+04$ & 839.93 & 215.82 & $1.03 \mathrm{E}+15$ & $45.75 \%$ & $3.15 \mathrm{E}+21$ \\
\hline & & 5.39 & $1.30 \mathrm{E}+04$ & 835.06 & 214.81 & $1.04 \mathrm{E}+15$ & $44.93 \%$ & $3.09 \mathrm{E}+21$ \\
\hline & & 4.15 & $1.31 \mathrm{E}+04$ & 840.09 & 216.04 & $1.05 \mathrm{E}+15$ & $45.19 \%$ & $3.11 \mathrm{E}+21$ \\
\hline & & 2.91 & $1.31 \mathrm{E}+04$ & 842.14 & 216.36 & $1.06 \mathrm{E}+15$ & $45.75 \%$ & $3.15 \mathrm{E}+21$ \\
\hline & & 1.68 & $1.33 \mathrm{E}+04$ & 854.97 & 218.94 & $1.06 \mathrm{E}+15$ & $47.71 \%$ & $3.31 \mathrm{E}+21$ \\
\hline \multirow{5}{*}{ B-4 } & \multirow{5}{*}{ L1H37Z } & -1.68 & $1.35 \mathrm{E}+04$ & 859.49 & 222.41 & $1.07 \mathrm{E}+15$ & $47.27 \%$ & $3.32 \mathrm{E}+21$ \\
\hline & & -2.91 & $1.32 \mathrm{E}+04$ & 836.72 & 217.14 & $1.06 \mathrm{E}+15$ & $45.69 \%$ & $3.19 \mathrm{E}+21$ \\
\hline & & -4.15 & $1.32 \mathrm{E}+04$ & 837.24 & 217.30 & $1.05 \mathrm{E}+15$ & $45.54 \%$ & $3.18 \mathrm{E}+21$ \\
\hline & & -5.39 & $1.31 \mathrm{E}+04$ & 834.71 & 216.78 & $1.05 \mathrm{E}+15$ & $45.17 \%$ & $3.15 \mathrm{E}+21$ \\
\hline & & -6.63 & $1.32 \mathrm{E}+04$ & 839.39 & 217.71 & $1.04 \mathrm{E}+15$ & $46.05 \%$ & $3.22 \mathrm{E}+21$ \\
\hline \multirow{5}{*}{ B-5 } & \multirow{5}{*}{ L1H38Z } & -8.93 & $1.24 \mathrm{E}+04$ & 828.50 & 204.81 & $1.01 \mathrm{E}+15$ & $45.02 \%$ & $2.98 \mathrm{E}+21$ \\
\hline & & -10.17 & $1.19 \mathrm{E}+04$ & 791.56 & 196.53 & $9.93 \mathrm{E}+14$ & $42.52 \%$ & $2.79 \mathrm{E}+21$ \\
\hline & & -11.41 & $1.18 \mathrm{E}+04$ & 781.09 & 194.27 & $9.71 \mathrm{E}+14$ & $41.38 \%$ & $2.71 \mathrm{E}+21$ \\
\hline & & -12.65 & $1.14 \mathrm{E}+04$ & 753.34 & 187.90 & $9.36 \mathrm{E}+14$ & $39.65 \%$ & $2.59 \mathrm{E}+21$ \\
\hline & & -13.89 & $1.13 \mathrm{E}+04$ & 745.39 & 186.06 & $9.00 \mathrm{E}+14$ & $39.28 \%$ & $2.56 \mathrm{E}+21$ \\
\hline \multirow{5}{*}{ B-6 } & \multirow{5}{*}{ L1H39Z } & -16.18 & $1.07 \mathrm{E}+04$ & 677.76 & 177.04 & $8.13 \mathrm{E}+14$ & $35.32 \%$ & $2.37 \mathrm{E}+21$ \\
\hline & & -17.42 & $9.75 \mathrm{E}+03$ & 613.03 & 161.01 & $7.51 \mathrm{E}+14$ & $31.89 \%$ & $2.12 \mathrm{E}+21$ \\
\hline & & -18.66 & $8.98 \mathrm{E}+03$ & 562.33 & 148.34 & $6.86 \mathrm{E}+14$ & $29.16 \%$ & $1.93 \mathrm{E}+21$ \\
\hline & & -19.90 & $8.19 \mathrm{E}+03$ & 510.31 & 135.17 & $6.15 \mathrm{E}+14$ & $26.55 \%$ & $1.74 \mathrm{E}+21$ \\
\hline & & -21.14 & $7.43 \mathrm{E}+03$ & 460.70 & 122.61 & $5.28 \mathrm{E}+14$ & $23.54 \%$ & $1.54 \mathrm{E}+21$ \\
\hline
\end{tabular}

(a) Elevations for MCNP cells are reported at cell centroids relative to the modeled core axial midplane at 24.0 inches. 
Table 20: Cycle 145A, MCNP-Calculated HGR, Neutron Flux, Depletion, and Fission Density for AFIP-4 Fuel Foils in Position A, 38.0 EFPD (MOC2), Center Lobe Power at 23.2 MW (89.7 EFPD Cumulative). ${ }^{3}$

\begin{tabular}{|c|c|c|c|c|c|c|c|c|}
\hline $\begin{array}{c}\text { Plate } \\
\text { ID }\end{array}$ & $\begin{array}{l}\text { Fuel } \\
\text { Plate } \\
\end{array}$ & $\begin{array}{c}\text { MCNP } \\
\text { Elevation }^{a} \\
\text { (inches) }^{a}\end{array}$ & $\begin{array}{l}\text { Fission } \\
\text { Power } \\
\text { Density } \\
\text { (W/cc) } \\
\end{array}$ & $\begin{array}{c}\text { Fission } \\
\text { Heat } \\
\text { Rate } \\
(W / g) \\
\end{array}$ & $\begin{array}{c}\text { Surface } \\
\text { Heat } \\
\text { Flux } \\
(\mathbf{W} / \mathbf{c m} 2) \\
\end{array}$ & $\begin{array}{c}\text { Neutron } \\
\text { Flux } \\
\text { (n/cm2sec) }\end{array}$ & $\begin{array}{c}\% \\
\text { Depletion } \\
\text { U-235 } \\
(\%) \\
\end{array}$ & $\begin{array}{c}\text { Fission } \\
\text { Density } \\
\text { (fissions/cc) }\end{array}$ \\
\hline \multirow{5}{*}{ A-1 } & \multirow{5}{*}{ L1B31Z } & 21.14 & $6.58 \mathrm{E}+03$ & 425.28 & 108.64 & $4.68 \mathrm{E}+14$ & $25.85 \%$ & $1.62 \mathrm{E}+21$ \\
\hline & & 19.90 & $7.50 \mathrm{E}+03$ & 487.31 & 123.75 & $5.55 \mathrm{E}+14$ & $29.63 \%$ & $1.87 \mathrm{E}+21$ \\
\hline & & 18.66 & $8.24 \mathrm{E}+03$ & 538.83 & 136.02 & $6.31 \mathrm{E}+14$ & $33.32 \%$ & $2.13 \mathrm{E}+21$ \\
\hline & & 17.42 & $9.02 \mathrm{E}+03$ & 593.30 & 148.98 & $7.05 \mathrm{E}+14$ & $36.65 \%$ & $2.36 \mathrm{E}+21$ \\
\hline & & 16.18 & $9.70 \mathrm{E}+03$ & 642.63 & 160.18 & $7.70 \mathrm{E}+14$ & $41.17 \%$ & $2.67 \mathrm{E}+21$ \\
\hline \multirow{5}{*}{ A-2 } & \multirow{5}{*}{ L1B33Z } & 13.89 & $1.15 \mathrm{E}+04$ & 711.44 & 189.79 & $8.75 \mathrm{E}+14$ & $45.45 \%$ & $3.22 \mathrm{E}+21$ \\
\hline & & 12.65 & $1.17 \mathrm{E}+04$ & 724.34 & 192.89 & $9.14 \mathrm{E}+14$ & $46.44 \%$ & $3.30 \mathrm{E}+21$ \\
\hline & & 11.41 & $1.18 \mathrm{E}+04$ & 735.54 & 195.18 & $9.53 \mathrm{E}+14$ & $48.57 \%$ & $3.47 \mathrm{E}+21$ \\
\hline & & 10.17 & $1.21 \mathrm{E}+04$ & 756.16 & 200.04 & $9.82 \mathrm{E}+14$ & $50.31 \%$ & $3.61 \mathrm{E}+21$ \\
\hline & & 8.93 & $1.24 \mathrm{E}+04$ & 777.20 & 204.54 & $1.00 \mathrm{E}+15$ & $53.32 \%$ & $3.86 \mathrm{E}+21$ \\
\hline \multirow{5}{*}{ A-3 } & \multirow{5}{*}{ L1B32Z } & 6.63 & $1.09 \mathrm{E}+04$ & 809.26 & 180.11 & $1.03 \mathrm{E}+15$ & $56.85 \%$ & $3.54 \mathrm{E}+21$ \\
\hline & & 5.39 & $1.09 \mathrm{E}+04$ & 809.64 & 180.43 & $1.03 \mathrm{E}+15$ & $56.02 \%$ & $3.49 \mathrm{E}+21$ \\
\hline & & 4.15 & $1.10 \mathrm{E}+04$ & 814.77 & 181.38 & $1.04 \mathrm{E}+15$ & $56.71 \%$ & $3.54 \mathrm{E}+21$ \\
\hline & & 2.91 & $1.09 \mathrm{E}+04$ & 807.33 & 179.52 & $1.04 \mathrm{E}+15$ & $57.32 \%$ & $3.58 \mathrm{E}+21$ \\
\hline & & 1.68 & $1.09 \mathrm{E}+04$ & 814.20 & 180.46 & $1.05 \mathrm{E}+15$ & $59.10 \%$ & $3.71 \mathrm{E}+21$ \\
\hline \multirow{5}{*}{ A-4 } & \multirow{5}{*}{ L1B51Z } & -1.68 & $1.23 \mathrm{E}+04$ & 803.60 & 203.07 & $1.05 \mathrm{E}+15$ & $57.59 \%$ & $4.09 \mathrm{E}+21$ \\
\hline & & -2.91 & $1.22 \mathrm{E}+04$ & 796.83 & 201.89 & $1.05 \mathrm{E}+15$ & $56.06 \%$ & $3.96 \mathrm{E}+21$ \\
\hline & & -4.15 & $1.22 \mathrm{E}+04$ & 794.17 & 201.23 & $1.05 \mathrm{E}+15$ & $56.00 \%$ & $3.96 \mathrm{E}+21$ \\
\hline & & -5.39 & $1.23 \mathrm{E}+04$ & 801.79 & 203.33 & $1.04 \mathrm{E}+15$ & $55.49 \%$ & $3.91 \mathrm{E}+21$ \\
\hline & & -6.63 & $1.22 \mathrm{E}+04$ & 795.39 & 201.47 & $1.04 \mathrm{E}+15$ & $56.27 \%$ & $3.98 \mathrm{E}+21$ \\
\hline \multirow{5}{*}{ A-5 } & \multirow{5}{*}{ L1B52Z } & -8.93 & $1.23 \mathrm{E}+04$ & 782.21 & 203.01 & $1.02 \mathrm{E}+15$ & $55.01 \%$ & $3.95 \mathrm{E}+21$ \\
\hline & & -10.17 & $1.22 \mathrm{E}+04$ & 772.52 & 201.43 & $1.00 \mathrm{E}+15$ & $52.23 \%$ & $3.72 \mathrm{E}+21$ \\
\hline & & -11.41 & $1.21 \mathrm{E}+04$ & 763.20 & 199.48 & $9.79 \mathrm{E}+14$ & $50.85 \%$ & $3.61 \mathrm{E}+21$ \\
\hline & & -12.65 & $1.17 \mathrm{E}+04$ & 736.35 & 193.03 & $9.49 \mathrm{E}+14$ & $49.16 \%$ & $3.47 \mathrm{E}+21$ \\
\hline & & -13.89 & $1.16 \mathrm{E}+04$ & 728.91 & 191.34 & $9.15 \mathrm{E}+14$ & $48.34 \%$ & $3.41 \mathrm{E}+21$ \\
\hline \multirow{5}{*}{ A-6 } & \multirow{5}{*}{ L1B53Z } & -16.18 & $1.07 \mathrm{E}+04$ & 679.11 & 177.19 & $8.27 \mathrm{E}+14$ & $44.25 \%$ & $3.05 \mathrm{E}+21$ \\
\hline & & -17.42 & $1.00 \mathrm{E}+04$ & 631.02 & 165.79 & $7.70 \mathrm{E}+14$ & $40.08 \%$ & $2.74 \mathrm{E}+21$ \\
\hline & & -18.66 & $9.34 \mathrm{E}+03$ & 583.57 & 154.16 & $7.08 \mathrm{E}+14$ & $36.85 \%$ & $2.50 \mathrm{E}+21$ \\
\hline & & -19.90 & $8.53 \mathrm{E}+03$ & 530.58 & 140.88 & $6.33 \mathrm{E}+14$ & $33.67 \%$ & $2.26 \mathrm{E}+21$ \\
\hline & & -21.14 & $7.76 \mathrm{E}+03$ & 479.77 & 128.13 & $5.47 \mathrm{E}+14$ & $30.02 \%$ & $2.00 \mathrm{E}+21$ \\
\hline
\end{tabular}

(a) Elevations for MCNP cells are reported at cell centroids relative to the modeled core axial midplane at 24.0 inches. 
Table 21: Cycle 145A, MCNP-Calculated HGR, Neutron Flux, Depletion, and Fission Density for AFIP-4 Fuel Foils in Position B, 38.0 EFPD (MOC2), Center Lobe Power at 23.2 MW (89.7 EFPD Cumulative). ${ }^{3}$

\begin{tabular}{|c|c|c|c|c|c|c|c|c|}
\hline $\begin{array}{c}\text { Plate } \\
\text { ID }\end{array}$ & $\begin{array}{l}\text { Fuel } \\
\text { Plate } \\
\end{array}$ & $\begin{array}{c}\text { MCNP } \\
\text { Elevation }^{\mathrm{a}} \\
\text { (inches) }^{-}\end{array}$ & $\begin{array}{l}\text { Fission } \\
\text { Power } \\
\text { Density } \\
\text { (W/cc) } \\
\end{array}$ & $\begin{array}{c}\text { Fission } \\
\text { Heat } \\
\text { Rate } \\
(\mathbf{W} / \mathrm{g}) \\
\end{array}$ & $\begin{array}{c}\text { Surface } \\
\text { Heat } \\
\text { Flux } \\
\text { (W/cm2) } \\
\end{array}$ & $\begin{array}{c}\text { Neutron } \\
\text { Flux } \\
\text { (n/cm2sec) } \\
\end{array}$ & $\begin{array}{c}\% \\
\text { Depletion } \\
\text { U-235 } \\
(\%) \\
\end{array}$ & $\begin{array}{c}\text { Fission } \\
\text { Density } \\
\text { (fissions/cc) }\end{array}$ \\
\hline \multirow{5}{*}{ B-1 } & \multirow{5}{*}{ L1H34Z } & 21.14 & $6.52 \mathrm{E}+03$ & 412.85 & 107.72 & $4.64 \mathrm{E}+14$ & $25.16 \%$ & $1.61 \mathrm{E}+21$ \\
\hline & & 19.90 & $7.45 \mathrm{E}+03$ & 474.36 & 123.04 & $5.50 \mathrm{E}+14$ & $28.83 \%$ & $1.86 \mathrm{E}+21$ \\
\hline & & 18.66 & $8.19 \mathrm{E}+03$ & 523.92 & 135.15 & $6.25 \mathrm{E}+14$ & $32.35 \%$ & $2.10 \mathrm{E}+21$ \\
\hline & & 17.42 & $8.86 \mathrm{E}+03$ & 570.25 & 146.24 & $6.93 \mathrm{E}+14$ & $36.00 \%$ & $2.36 \mathrm{E}+21$ \\
\hline & & 16.18 & $9.71 \mathrm{E}+03$ & 629.64 & 160.33 & $7.60 \mathrm{E}+14$ & $40.33 \%$ & $2.67 \mathrm{E}+21$ \\
\hline \multirow{5}{*}{ B-2 } & \multirow{5}{*}{ L1H35Z } & 13.89 & $1.06 \mathrm{E}+04$ & 693.21 & 175.50 & $8.62 \mathrm{E}+14$ & $45.05 \%$ & $3.02 \mathrm{E}+21$ \\
\hline & & 12.65 & $1.09 \mathrm{E}+04$ & 709.56 & 179.36 & $9.03 \mathrm{E}+14$ & $45.87 \%$ & $3.08 \mathrm{E}+21$ \\
\hline & & 11.41 & $1.12 \mathrm{E}+04$ & 733.89 & 184.86 & $9.42 \mathrm{E}+14$ & $48.01 \%$ & $3.24 \mathrm{E}+21$ \\
\hline & & 10.17 & $1.13 \mathrm{E}+04$ & 745.37 & 187.15 & $9.68 \mathrm{E}+14$ & $49.79 \%$ & $3.39 \mathrm{E}+21$ \\
\hline & & 8.93 & $1.16 \mathrm{E}+04$ & 769.89 & 192.31 & $9.95 \mathrm{E}+14$ & $52.91 \%$ & $3.62 \mathrm{E}+21$ \\
\hline \multirow{5}{*}{ B-3 } & \multirow{5}{*}{ L1H36Z } & 6.63 & $1.20 \mathrm{E}+04$ & 781.50 & 197.49 & $1.02 \mathrm{E}+15$ & $55.33 \%$ & $3.87 \mathrm{E}+21$ \\
\hline & & 5.39 & $1.20 \mathrm{E}+04$ & 786.11 & 198.91 & $1.03 \mathrm{E}+15$ & $54.45 \%$ & $3.81 \mathrm{E}+21$ \\
\hline & & 4.15 & $1.21 \mathrm{E}+04$ & 787.62 & 199.19 & $1.03 \mathrm{E}+15$ & $54.76 \%$ & $3.83 \mathrm{E}+21$ \\
\hline & & 2.91 & $1.20 \mathrm{E}+04$ & 787.12 & 198.89 & $1.04 \mathrm{E}+15$ & $55.31 \%$ & $3.87 \mathrm{E}+21$ \\
\hline & & 1.68 & $1.21 \mathrm{E}+04$ & 793.83 & 199.92 & $1.04 \mathrm{E}+15$ & $57.32 \%$ & $4.03 \mathrm{E}+21$ \\
\hline \multirow{5}{*}{ B-4 } & \multirow{5}{*}{ L1H37Z } & -1.68 & $1.23 \mathrm{E}+04$ & 797.89 & 203.03 & $1.04 \mathrm{E}+15$ & $56.91 \%$ & $4.06 \mathrm{E}+21$ \\
\hline & & -2.91 & $1.21 \mathrm{E}+04$ & 780.99 & 199.35 & $1.04 \mathrm{E}+15$ & $55.18 \%$ & $3.91 \mathrm{E}+21$ \\
\hline & & -4.15 & $1.21 \mathrm{E}+04$ & 783.13 & 199.92 & $1.04 \mathrm{E}+15$ & $55.02 \%$ & $3.90 \mathrm{E}+21$ \\
\hline & & -5.39 & $1.20 \mathrm{E}+04$ & 778.14 & 198.76 & $1.03 \mathrm{E}+15$ & $54.63 \%$ & $3.87 \mathrm{E}+21$ \\
\hline & & -6.63 & $1.21 \mathrm{E}+04$ & 783.24 & 199.80 & $1.03 \mathrm{E}+15$ & $55.55 \%$ & $3.94 \mathrm{E}+21$ \\
\hline \multirow{5}{*}{ B-5 } & \multirow{5}{*}{ L1H38Z } & -8.93 & $1.15 \mathrm{E}+04$ & 779.93 & 189.67 & $1.00 \mathrm{E}+15$ & $54.48 \%$ & $3.66 \mathrm{E}+21$ \\
\hline & & -10.17 & $1.13 \mathrm{E}+04$ & 760.89 & 185.96 & $9.89 \mathrm{E}+14$ & $51.73 \%$ & $3.45 \mathrm{E}+21$ \\
\hline & & -11.41 & $1.11 \mathrm{E}+04$ & 749.50 & 183.55 & $9.68 \mathrm{E}+14$ & $50.52 \%$ & $3.36 \mathrm{E}+21$ \\
\hline & & -12.65 & $1.09 \mathrm{E}+04$ & 733.86 & 180.28 & $9.39 \mathrm{E}+14$ & $48.55 \%$ & $3.21 \mathrm{E}+21$ \\
\hline & & -13.89 & $1.07 \mathrm{E}+04$ & 716.07 & 176.09 & $9.01 \mathrm{E}+14$ & $48.12 \%$ & $3.18 \mathrm{E}+21$ \\
\hline \multirow{5}{*}{ B-6 } & \multirow{5}{*}{ L1H39Z } & -16.18 & $1.03 \mathrm{E}+04$ & 662.21 & 170.62 & $8.20 \mathrm{E}+14$ & $43.60 \%$ & $2.96 \mathrm{E}+21$ \\
\hline & & -17.42 & $9.65 \mathrm{E}+03$ & 614.36 & 159.37 & $7.61 \mathrm{E}+14$ & $39.54 \%$ & $2.66 \mathrm{E}+21$ \\
\hline & & -18.66 & $8.93 \mathrm{E}+03$ & 565.54 & 147.49 & $6.99 \mathrm{E}+14$ & $36.28 \%$ & $2.42 \mathrm{E}+21$ \\
\hline & & -19.90 & $8.26 \mathrm{E}+03$ & 520.37 & 136.41 & $6.24 \mathrm{E}+14$ & $33.12 \%$ & $2.19 \mathrm{E}+21$ \\
\hline & & -21.14 & $7.60 \mathrm{E}+03$ & 475.72 & 125.42 & $5.44 \mathrm{E}+14$ & $29.58 \%$ & $1.94 \mathrm{E}+21$ \\
\hline
\end{tabular}

(a) Elevations for MCNP cells are reported at cell centroids relative to the modeled core axial midplane at 24.0 inches. 
Table 22: Cycle 145A, MCNP-Calculated HGR, Neutron Flux, Depletion, and Fission Density for AFIP-4 Fuel Foils in Position A, 54.7 EFPD (EOC), Center Lobe Power at 23.2 MW (106.4 EFPD Cumulative). ${ }^{3}$

\begin{tabular}{|c|c|c|c|c|c|c|c|c|}
\hline $\begin{array}{c}\text { Plate } \\
\text { ID } \\
\end{array}$ & $\begin{array}{l}\text { Fuel } \\
\text { Plate }\end{array}$ & $\begin{array}{c}\text { MCNP } \\
\text { Elevation }^{a} \\
\text { (inches) }\end{array}$ & $\begin{array}{l}\text { Fission } \\
\text { Power } \\
\text { Density } \\
\text { (W/cc) } \\
\end{array}$ & $\begin{array}{c}\text { Fission } \\
\text { Heat } \\
\text { Rate } \\
(\mathrm{W} / \mathrm{g}) \\
\end{array}$ & $\begin{array}{c}\text { Surface } \\
\text { Heat } \\
\text { Flux } \\
(W / c m 2) \\
\end{array}$ & $\begin{array}{c}\text { Neutron } \\
\text { Flux } \\
\text { (n/cm2sec) } \\
\end{array}$ & $\begin{array}{c}\% \\
\text { Depletion } \\
\text { U-235 } \\
(\%) \\
\end{array}$ & $\begin{array}{c}\text { Fission } \\
\text { Density } \\
\text { (fissions/cc) }\end{array}$ \\
\hline \multirow{5}{*}{ A-1 } & \multirow{5}{*}{ L1B31Z } & 21.14 & $6.48 \mathrm{E}+03$ & 421.94 & 106.97 & $4.69 \mathrm{E}+14$ & $30.68 \%$ & $1.94 \mathrm{E}+21$ \\
\hline & & 19.90 & $7.32 \mathrm{E}+03$ & 480.44 & 120.94 & $5.56 \mathrm{E}+14$ & $35.03 \%$ & $2.23 \mathrm{E}+21$ \\
\hline & & 18.66 & $8.01 \mathrm{E}+03$ & 529.20 & 132.33 & $6.32 \mathrm{E}+14$ & $39.19 \%$ & $2.52 \mathrm{E}+21$ \\
\hline & & 17.42 & $8.71 \mathrm{E}+03$ & 579.12 & 143.88 & $7.06 \mathrm{E}+14$ & $42.99 \%$ & $2.79 \mathrm{E}+21$ \\
\hline & & 16.18 & $9.27 \mathrm{E}+03$ & 621.38 & 153.12 & $7.72 \mathrm{E}+14$ & $47.86 \%$ & $3.14 \mathrm{E}+21$ \\
\hline \multirow{5}{*}{ A-2 } & \multirow{5}{*}{ L1B33Z } & 13.89 & $1.08 \mathrm{E}+04$ & 679.77 & 179.10 & $8.77 \mathrm{E}+14$ & $52.63 \%$ & $3.77 \mathrm{E}+21$ \\
\hline & & 12.65 & $1.10 \mathrm{E}+04$ & 692.23 & 182.04 & $9.17 \mathrm{E}+14$ & $53.71 \%$ & $3.85 \mathrm{E}+21$ \\
\hline & & 11.41 & $1.11 \mathrm{E}+04$ & 697.97 & 182.84 & $9.53 \mathrm{E}+14$ & $55.85 \%$ & $4.04 \mathrm{E}+21$ \\
\hline & & 10.17 & $1.13 \mathrm{E}+04$ & 716.40 & 187.05 & $9.84 \mathrm{E}+14$ & $57.70 \%$ & $4.19 \mathrm{E}+21$ \\
\hline & & 8.93 & $1.15 \mathrm{E}+04$ & 730.29 & 189.63 & $1.00 \mathrm{E}+15$ & $60.75 \%$ & $4.45 \mathrm{E}+21$ \\
\hline \multirow{5}{*}{ A-3 } & \multirow{5}{*}{ L1B32Z } & 6.63 & $9.96 \mathrm{E}+03$ & 749.41 & 164.49 & $1.03 \mathrm{E}+15$ & $64.35 \%$ & $4.06 \mathrm{E}+21$ \\
\hline & & 5.39 & $1.00 \mathrm{E}+04$ & 752.16 & 165.30 & $1.04 \mathrm{E}+15$ & $63.54 \%$ & $4.01 \mathrm{E}+21$ \\
\hline & & 4.15 & $1.01 \mathrm{E}+04$ & 756.47 & 166.04 & $1.04 \mathrm{E}+15$ & $64.24 \%$ & $4.06 \mathrm{E}+21$ \\
\hline & & 2.91 & $9.97 \mathrm{E}+03$ & 750.46 & 164.55 & $1.05 \mathrm{E}+15$ & $64.78 \%$ & $4.10 \mathrm{E}+21$ \\
\hline & & 1.68 & $9.92 \mathrm{E}+03$ & 749.30 & 163.79 & $1.05 \mathrm{E}+15$ & $66.51 \%$ & $4.23 \mathrm{E}+21$ \\
\hline \multirow{5}{*}{ A-4 } & \multirow{5}{*}{ L1B51Z } & -1.68 & $1.13 \mathrm{E}+04$ & 745.94 & 185.90 & $1.06 \mathrm{E}+15$ & $65.02 \%$ & $4.67 \mathrm{E}+21$ \\
\hline & & -2.91 & $1.13 \mathrm{E}+04$ & 745.74 & 186.35 & $1.05 \mathrm{E}+15$ & $63.51 \%$ & $4.55 \mathrm{E}+21$ \\
\hline & & -4.15 & $1.13 \mathrm{E}+04$ & 744.38 & 186.03 & $1.05 \mathrm{E}+15$ & $63.43 \%$ & $4.54 \mathrm{E}+21$ \\
\hline & & -5.39 & $1.13 \mathrm{E}+04$ & 747.89 & 187.07 & $1.05 \mathrm{E}+15$ & $63.02 \%$ & $4.50 \mathrm{E}+21$ \\
\hline & & -6.63 & $1.12 \mathrm{E}+04$ & 740.89 & 185.09 & $1.04 \mathrm{E}+15$ & $63.72 \%$ & $4.56 \mathrm{E}+21$ \\
\hline \multirow{5}{*}{ A-5 } & \multirow{5}{*}{ L1B52Z } & -8.93 & $1.14 \mathrm{E}+04$ & 732.56 & 187.56 & $1.02 \mathrm{E}+15$ & $62.41 \%$ & $4.53 \mathrm{E}+21$ \\
\hline & & -10.17 & $1.13 \mathrm{E}+04$ & 726.72 & 186.96 & $1.00 \mathrm{E}+15$ & $59.67 \%$ & $4.31 \mathrm{E}+21$ \\
\hline & & -11.41 & $1.12 \mathrm{E}+04$ & 719.66 & 185.61 & $9.80 \mathrm{E}+14$ & $58.26 \%$ & $4.19 \mathrm{E}+21$ \\
\hline & & -12.65 & $1.10 \mathrm{E}+04$ & 703.93 & 182.18 & $9.53 \mathrm{E}+14$ & $56.40 \%$ & $4.03 \mathrm{E}+21$ \\
\hline & & -13.89 & $1.09 \mathrm{E}+04$ & 693.51 & 179.77 & $9.18 \mathrm{E}+14$ & $55.58 \%$ & $3.96 \mathrm{E}+21$ \\
\hline \multirow{5}{*}{ A-6 } & \multirow{5}{*}{ L1B53Z } & -16.18 & $1.02 \mathrm{E}+04$ & 650.26 & 167.66 & $8.29 \mathrm{E}+14$ & $51.15 \%$ & $3.56 \mathrm{E}+21$ \\
\hline & & -17.42 & $9.65 \mathrm{E}+03$ & 612.96 & 159.27 & $7.72 \mathrm{E}+14$ & $46.68 \%$ & $3.22 \mathrm{E}+21$ \\
\hline & & -18.66 & $9.06 \mathrm{E}+03$ & 571.88 & 149.51 & $7.10 \mathrm{E}+14$ & $43.06 \%$ & $2.94 \mathrm{E}+21$ \\
\hline & & -19.90 & $8.32 \mathrm{E}+03$ & 522.52 & 137.43 & $6.35 \mathrm{E}+14$ & $39.45 \%$ & $2.67 \mathrm{E}+21$ \\
\hline & & -21.14 & $7.59 \mathrm{E}+03$ & 473.32 & 125.34 & $5.50 \mathrm{E}+14$ & $35.36 \%$ & $2.37 \mathrm{E}+21$ \\
\hline
\end{tabular}

(a) Elevations for MCNP cells are reported at cell centroids relative to the modeled core axial midplane at 24.0 inches. 
Table 23: Cycle 145A, MCNP-Calculated HGR, Neutron Flux, Depletion, and Fission Density for AFIP-4 Fuel Foils in Position B, 54.7 EFPD (EOC), Center Lobe Power at 23.2 MW (106.4 EFPD Cumulative). ${ }^{3}$

\begin{tabular}{|c|c|c|c|c|c|c|c|c|}
\hline $\begin{array}{c}\text { Plate } \\
\text { ID }\end{array}$ & $\begin{array}{l}\text { Fuel } \\
\text { Plate }\end{array}$ & $\begin{array}{c}\text { MCNP } \\
\text { Elevation }^{a} \\
\text { (inches) }\end{array}$ & $\begin{array}{c}\text { Fission } \\
\text { Power } \\
\text { Density } \\
\text { (W/cc) } \\
\end{array}$ & $\begin{array}{c}\text { Fission } \\
\text { Heat } \\
\text { Rate } \\
(\mathrm{W} / \mathrm{g}) \\
\end{array}$ & $\begin{array}{c}\text { Surface } \\
\text { Heat } \\
\text { Flux } \\
(W / c m 2) \\
\end{array}$ & $\begin{array}{c}\text { Neutron } \\
\text { Flux } \\
\text { (n/cm2sec) } \\
\end{array}$ & $\begin{array}{c}\% \\
\text { Depletion } \\
\text { U-235 } \\
(\%) \\
\end{array}$ & $\begin{array}{c}\text { Fission } \\
\text { Density } \\
\text { (fissions/ce) }\end{array}$ \\
\hline \multirow{5}{*}{ B-1 } & \multirow{5}{*}{ L1H34Z } & 21.14 & $6.40 \mathrm{E}+03$ & 407.94 & 105.65 & $4.63 \mathrm{E}+14$ & $29.86 \%$ & $1.92 \mathrm{E}+21$ \\
\hline & & 19.90 & $7.28 \mathrm{E}+03$ & 467.46 & 120.24 & $5.50 \mathrm{E}+14$ & $34.13 \%$ & $2.21 \mathrm{E}+21$ \\
\hline & & 18.66 & $7.92 \mathrm{E}+03$ & 511.47 & 130.71 & $6.25 \mathrm{E}+14$ & $38.07 \%$ & $2.49 \mathrm{E}+21$ \\
\hline & & 17.42 & $8.55 \mathrm{E}+03$ & 555.93 & 141.12 & $6.93 \mathrm{E}+14$ & $42.11 \%$ & $2.78 \mathrm{E}+21$ \\
\hline & & 16.18 & $9.28 \mathrm{E}+03$ & 608.21 & 153.15 & $7.60 \mathrm{E}+14$ & $46.90 \%$ & $3.13 \mathrm{E}+21$ \\
\hline \multirow{5}{*}{ B-2 } & \multirow{5}{*}{ L1H35Z } & 13.89 & $9.99 \mathrm{E}+03$ & 659.53 & 164.94 & $8.62 \mathrm{E}+14$ & $52.07 \%$ & $3.53 \mathrm{E}+21$ \\
\hline & & 12.65 & $1.02 \mathrm{E}+04$ & 674.32 & 168.34 & $9.00 \mathrm{E}+14$ & $53.02 \%$ & $3.60 \mathrm{E}+21$ \\
\hline & & 11.41 & $1.04 \mathrm{E}+04$ & 691.84 & 172.04 & $9.42 \mathrm{E}+14$ & $55.29 \%$ & $3.78 \mathrm{E}+21$ \\
\hline & & 10.17 & $1.05 \mathrm{E}+04$ & 702.44 & 174.09 & $9.67 \mathrm{E}+14$ & $57.07 \%$ & $3.93 \mathrm{E}+21$ \\
\hline & & 8.93 & $1.07 \mathrm{E}+04$ & 716.08 & 176.50 & $9.94 \mathrm{E}+14$ & $60.30 \%$ & $4.18 \mathrm{E}+21$ \\
\hline \multirow{5}{*}{ B-3 } & \multirow{5}{*}{ L1H36Z } & 6.63 & $1.09 \mathrm{E}+04$ & 722.61 & 180.16 & $1.02 \mathrm{E}+15$ & $62.72 \%$ & $4.44 \mathrm{E}+21$ \\
\hline & & 5.39 & $1.11 \mathrm{E}+04$ & 731.02 & 182.48 & $1.03 \mathrm{E}+15$ & $61.87 \%$ & $4.38 \mathrm{E}+21$ \\
\hline & & 4.15 & $1.11 \mathrm{E}+04$ & 732.11 & 182.66 & $1.03 \mathrm{E}+15$ & $62.20 \%$ & $4.40 \mathrm{E}+21$ \\
\hline & & 2.91 & $1.10 \mathrm{E}+04$ & 730.22 & 182.03 & $1.04 \mathrm{E}+15$ & $62.74 \%$ & $4.44 \mathrm{E}+21$ \\
\hline & & 1.68 & $1.10 \mathrm{E}+04$ & 729.63 & 181.24 & $1.04 \mathrm{E}+15$ & $64.72 \%$ & $4.61 \mathrm{E}+21$ \\
\hline \multirow{5}{*}{ B-4 } & \multirow{5}{*}{ L1H37Z } & -1.68 & $1.12 \mathrm{E}+04$ & 733.97 & 184.21 & $1.04 \mathrm{E}+15$ & $64.31 \%$ & $4.64 \mathrm{E}+21$ \\
\hline & & -2.91 & $1.11 \mathrm{E}+04$ & 727.18 & 183.12 & $1.04 \mathrm{E}+15$ & $62.54 \%$ & $4.49 \mathrm{E}+21$ \\
\hline & & -4.15 & $1.11 \mathrm{E}+04$ & 729.52 & 183.72 & $1.04 \mathrm{E}+15$ & $62.38 \%$ & $4.48 \mathrm{E}+21$ \\
\hline & & -5.39 & $1.11 \mathrm{E}+04$ & 724.73 & 182.64 & $1.03 \mathrm{E}+15$ & $61.97 \%$ & $4.45 \mathrm{E}+21$ \\
\hline & & -6.63 & $1.11 \mathrm{E}+04$ & 727.35 & 183.03 & $1.03 \mathrm{E}+15$ & $62.92 \%$ & $4.52 \mathrm{E}+21$ \\
\hline \multirow{5}{*}{ B-5 } & \multirow{5}{*}{ L1H38Z } & -8.93 & $1.05 \mathrm{E}+04$ & 725.40 & 174.06 & $1.00 \mathrm{E}+15$ & $61.85 \%$ & $4.21 \mathrm{E}+21$ \\
\hline & & -10.17 & $1.04 \mathrm{E}+04$ & 713.98 & 172.20 & $9.90 \mathrm{E}+14$ & $59.10 \%$ & $3.98 \mathrm{E}+21$ \\
\hline & & -11.41 & $1.03 \mathrm{E}+04$ & 704.06 & 170.18 & $9.67 \mathrm{E}+14$ & $57.83 \%$ & $3.89 \mathrm{E}+21$ \\
\hline & & -12.65 & $1.02 \mathrm{E}+04$ & 695.44 & 168.68 & $9.39 \mathrm{E}+14$ & $55.78 \%$ & $3.73 \mathrm{E}+21$ \\
\hline & & -13.89 & $9.98 \mathrm{E}+03$ & 678.43 & 164.76 & $9.01 \mathrm{E}+14$ & $55.24 \%$ & $3.68 \mathrm{E}+21$ \\
\hline \multirow{5}{*}{ B-6 } & \multirow{5}{*}{ L1H39Z } & -16.18 & $9.76 \mathrm{E}+03$ & 632.38 & 161.07 & $8.19 \mathrm{E}+14$ & $50.37 \%$ & $3.45 \mathrm{E}+21$ \\
\hline & & -17.42 & $9.22 \mathrm{E}+03$ & 592.89 & 152.15 & $7.60 \mathrm{E}+14$ & $45.98 \%$ & $3.12 \mathrm{E}+21$ \\
\hline & & -18.66 & $8.62 \mathrm{E}+03$ & 551.01 & 142.26 & $7.00 \mathrm{E}+14$ & $42.34 \%$ & $2.85 \mathrm{E}+21$ \\
\hline & & -19.90 & $8.03 \mathrm{E}+03$ & 510.37 & 132.56 & $6.25 \mathrm{E}+14$ & $38.78 \%$ & $2.59 \mathrm{E}+21$ \\
\hline & & -21.14 & $7.38 \mathrm{E}+03$ & 466.09 & 121.84 & $5.44 \mathrm{E}+14$ & $34.88 \%$ & $2.31 \mathrm{E}+21$ \\
\hline
\end{tabular}

(a) Elevations for MCNP cells are reported at cell centroids relative to the modeled core axial midplane at 24.0 inches. 


\subsection{Axial Gradient}

The axial gradient is represented by the neutron flux profiles for plates in position A and B as shown in Figure 9 and Figure 10, respectively, and the fission density profiles for plates in position A and B as shown in Figure 11 and Figure 12, respectively. Since the AFIP-4 experiment was irradiated in the CFT, the transverse (radial) profile is relatively flat across the plates and was not reported.

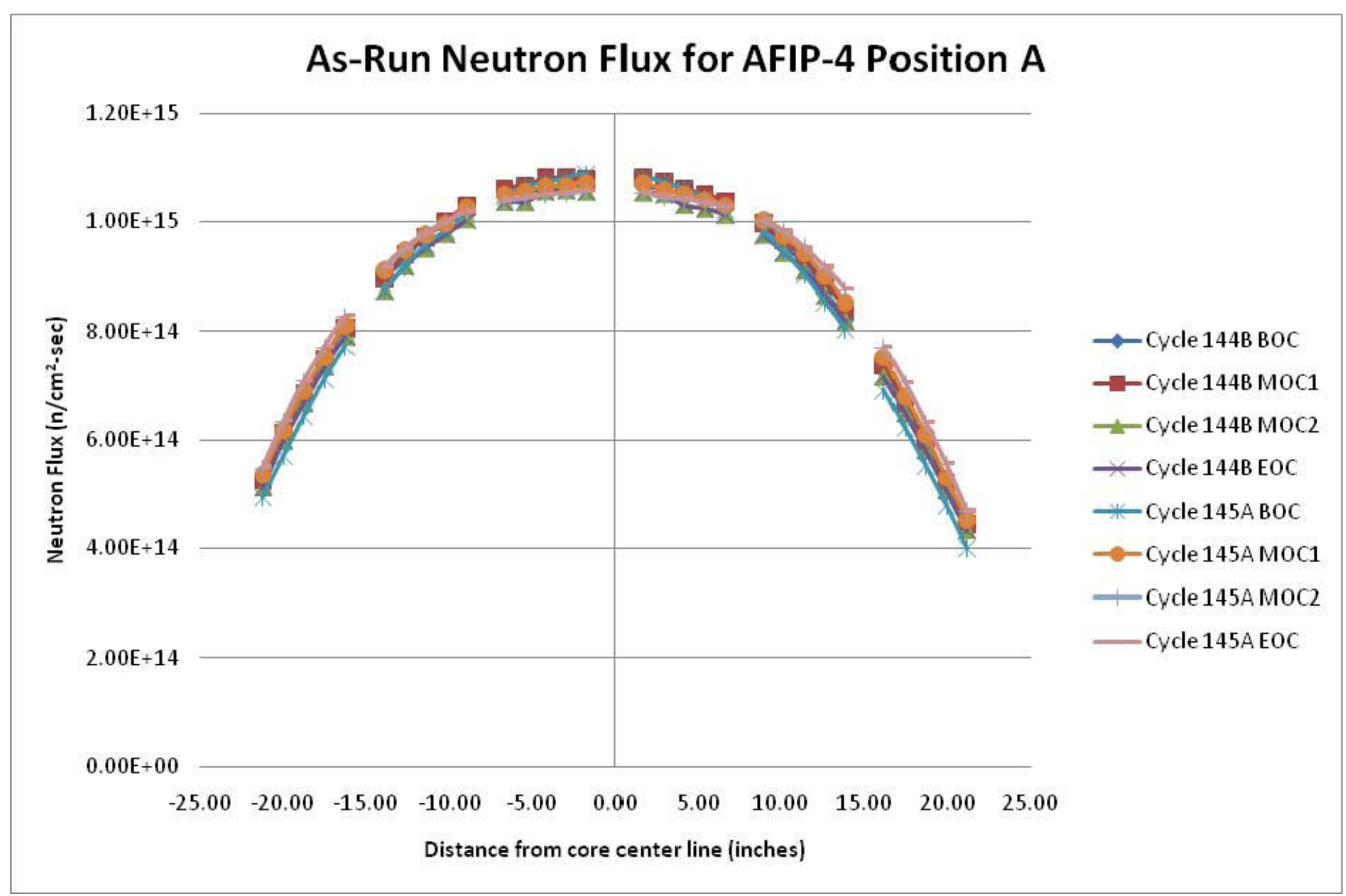

Figure 9: AFIP-4 Axial Neutron Flux Profile for Plates in Position A. ${ }^{3}$ 


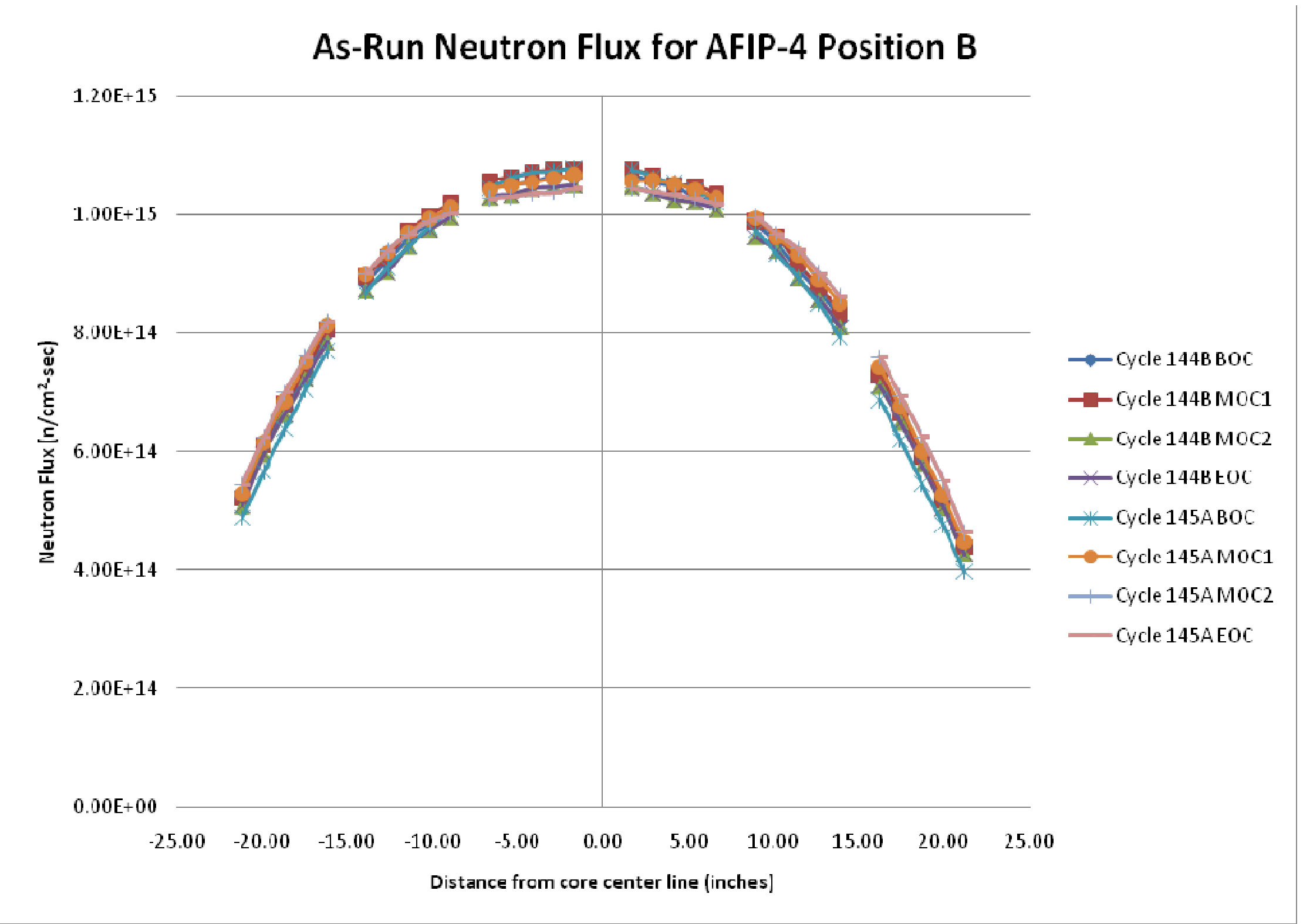

Figure 10: AFIP-4 Axial Neutron Flux Profile for Plates in Position B. ${ }^{3}$ 


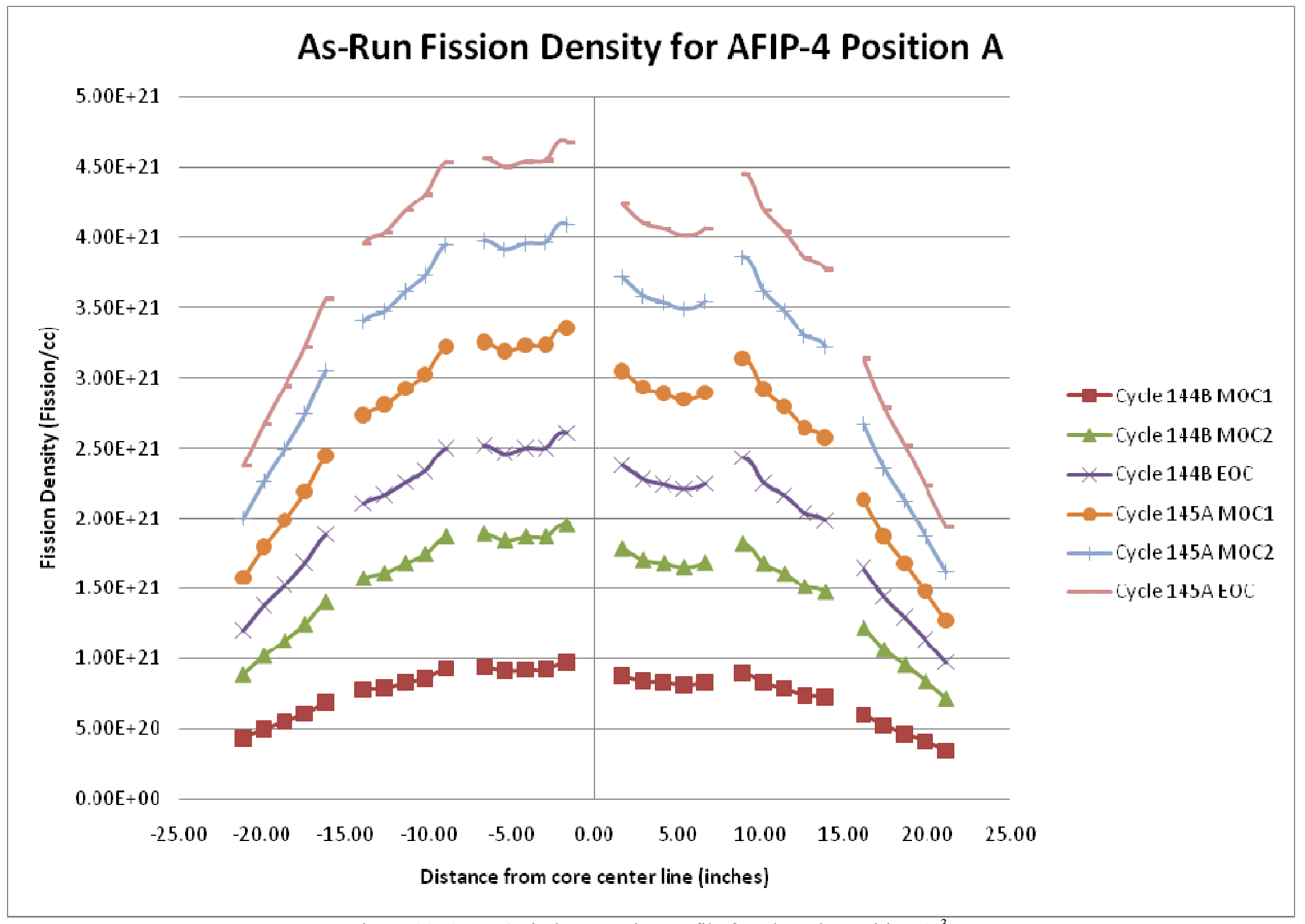

Figure 11: AFIP-4 Fission Density Profile for Plates in Position A. ${ }^{3}$ 


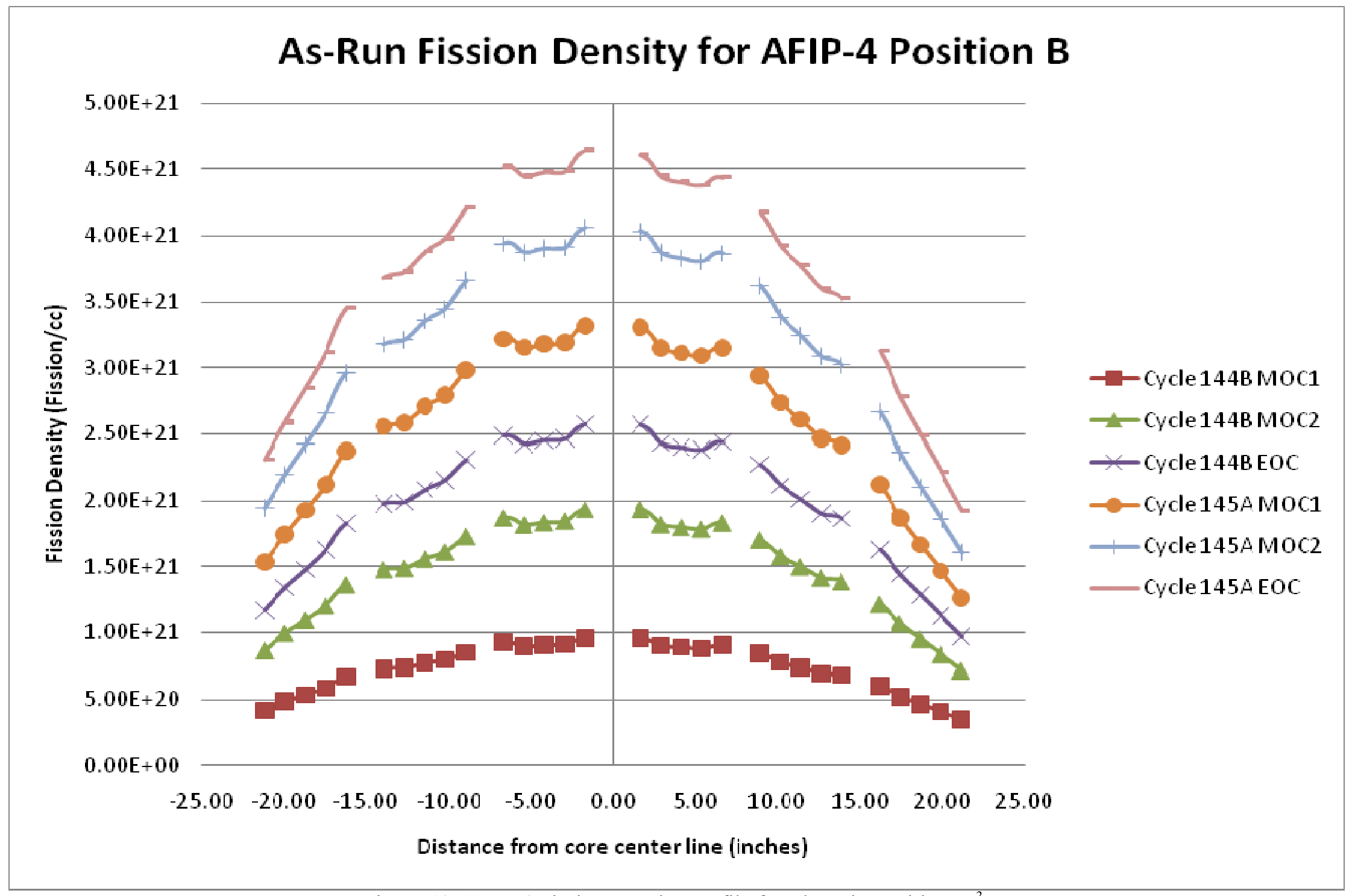

Figure 12: AFIP-4 Fission Density Profile for Plates in Position B. ${ }^{3}$ 


\section{HYDRAULIC TESTING}

Flow tests were performed to characterize the test assembly designed to irradiate full-size plates for the RERTR program in the ATR. The holder assembly design was used for several plate tests that were conducted in the CFT position ${ }^{5}$.

The test apparatus was designed and constructed to simulate the ATR CFT position geometry. The holder was fabricated such that the orifice plate on the bottom of the test train could be screwed on (rather than welded) to allow variation of the orifice diameter. The results of the flow tests were used to generate estimates of the coolant velocity and flow rate and are reported in Table $24^{5}$.

Table 24: AFIP irradiation vehicle flow conditions for each orifice configuration ${ }^{5}$.

\begin{tabular}{|c|c|c|c|c|}
\hline Configuration & $\begin{array}{c}\text { Total Internal } \\
\text { Loss } \\
\text { Coefficient }\end{array}$ & $\begin{array}{c}\text { Total Holder } \\
\text { Internal Flow } \\
(\mathrm{gpm})\end{array}$ & $\begin{array}{c}\text { Channel } \\
\text { Coolant Flow } \\
(\mathrm{gpm})\end{array}$ & $\begin{array}{c}\text { Channel } \\
\text { Coolant } \\
\text { Velocity }(\mathrm{m} / \mathrm{s})\end{array}$ \\
\hline $6 \mathrm{~mm}$ orifice & 0.1709 & 20.8 & 6.6 & 1.7 \\
\hline $7 \mathrm{~mm}$ orifice & 0.1203 & 24.8 & 7.9 & 2.0 \\
\hline $8 \mathrm{~mm}$ orifice & 0.0869 & 29.2 & 9.3 & 2.4 \\
\hline $8.1 \mathrm{~mm}$ orifice & 0.0861 & 29.3 & 9.3 & 2.4 \\
\hline $9 \mathrm{~mm}$ orifice & 0.0680 & 33.0 & 10.5 & 2.7 \\
\hline $10 \mathrm{~mm}$ orifice & 0.0568 & 36.1 & 11.5 & 3.0 \\
\hline Open (no orifice) & 0.00164 & 212.5 & 67.5 & 17.4 \\
\hline
\end{tabular}

Based on the results from the hydraulic testing, the orifice was sized to 0.8125 inches $(20.64 \mathrm{~mm})$ to provide a coolant channel velocity of approximately $10.36 \mathrm{~m} / \mathrm{s}$ for two pump operation ${ }^{6}$. 


\section{AS-RUN THERMAL ANALYSIS}

The thermal as-run analysis was performed using the as-built geometry, MCNP-calculated surface heat flux $\left(\mathrm{W} / \mathrm{cm}^{2}\right)$ and nominal coolant channel flow rate. ABAQUS ${ }^{7}$ was used to calculate the coolant channel temperatures and plate surface temperatures.

The heat transfer correlation used to calculate these temperatures was calculated from the Colburn equation (equation 5-50c from Reference 8):

$$
N u=\frac{h D}{k}=0.023 R e^{0.8} \operatorname{Pr}^{0.3}
$$

Where $\mathrm{Nu}$ is the Nusselt number, $\mathrm{h}$ is the heat transfer coefficient, $\mathrm{D}$ is the hydraulic diameter, $\mathrm{k}$ is the thermal conductivity, Re is the Reynolds number and Pr is the Prandlt number.

\subsection{Coolant Channel Temperature}

The coolant temperature was analyzed at the three flow channels in the test assembly. The left coolant channel is west of plate position $\mathrm{B}$, the center coolant channel is in between plate positions $\mathrm{A}$ and $\mathrm{B}$, and the right coolant channel is in between plate position $\mathrm{A}$ and the Ram Rod (refer to Figure 1). For each cycle interval, the coolant temperature was plotted as a function of location along the test assembly with 0.0 inches being at the top of the assembly. These plots are show in Figure 13 through Figure 20.

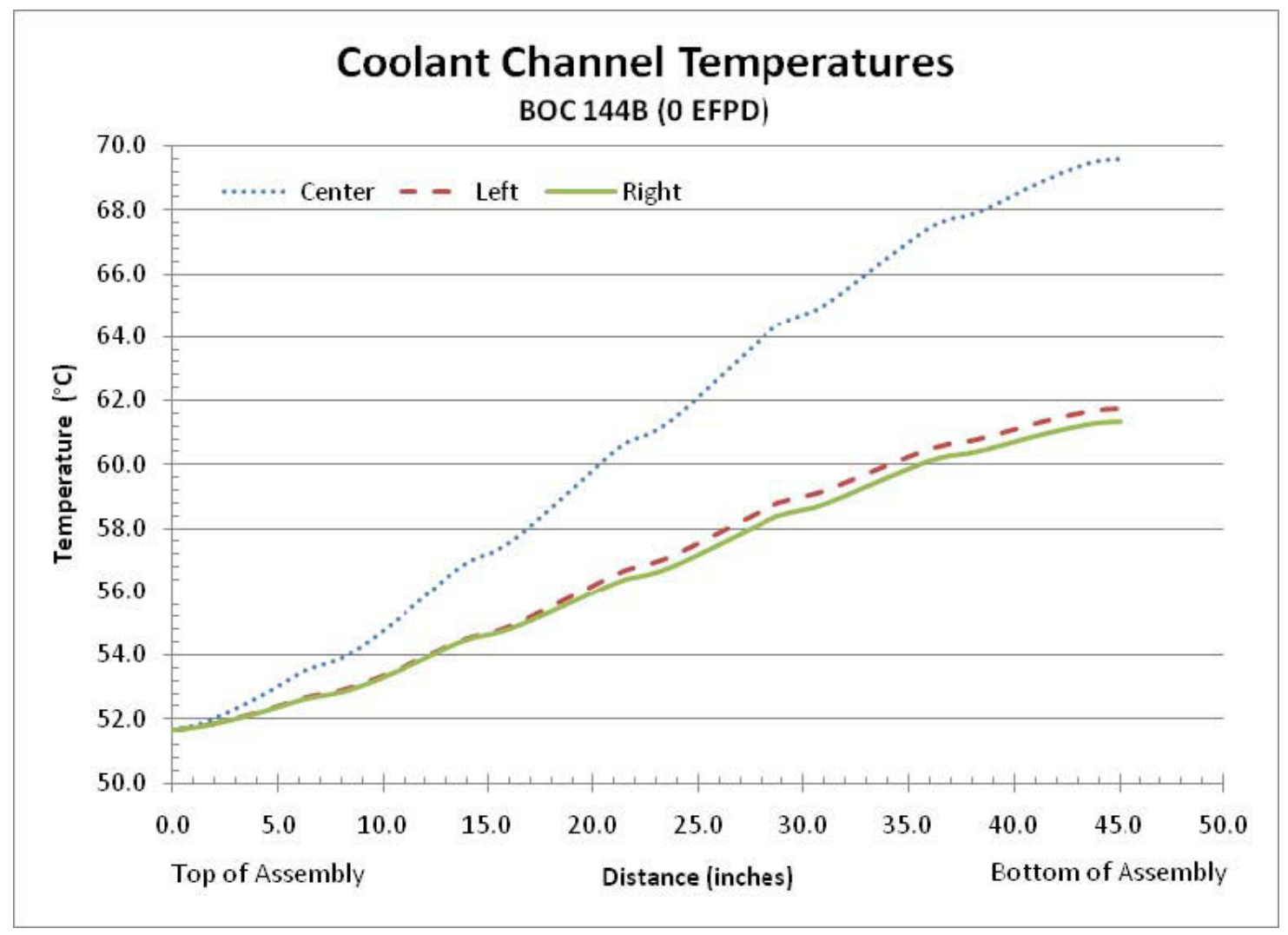

Figure 13: Coolant channel temperatures as a function of location along the AFIP-4 test assembly at BOC 144B (0.0 EFPD). 


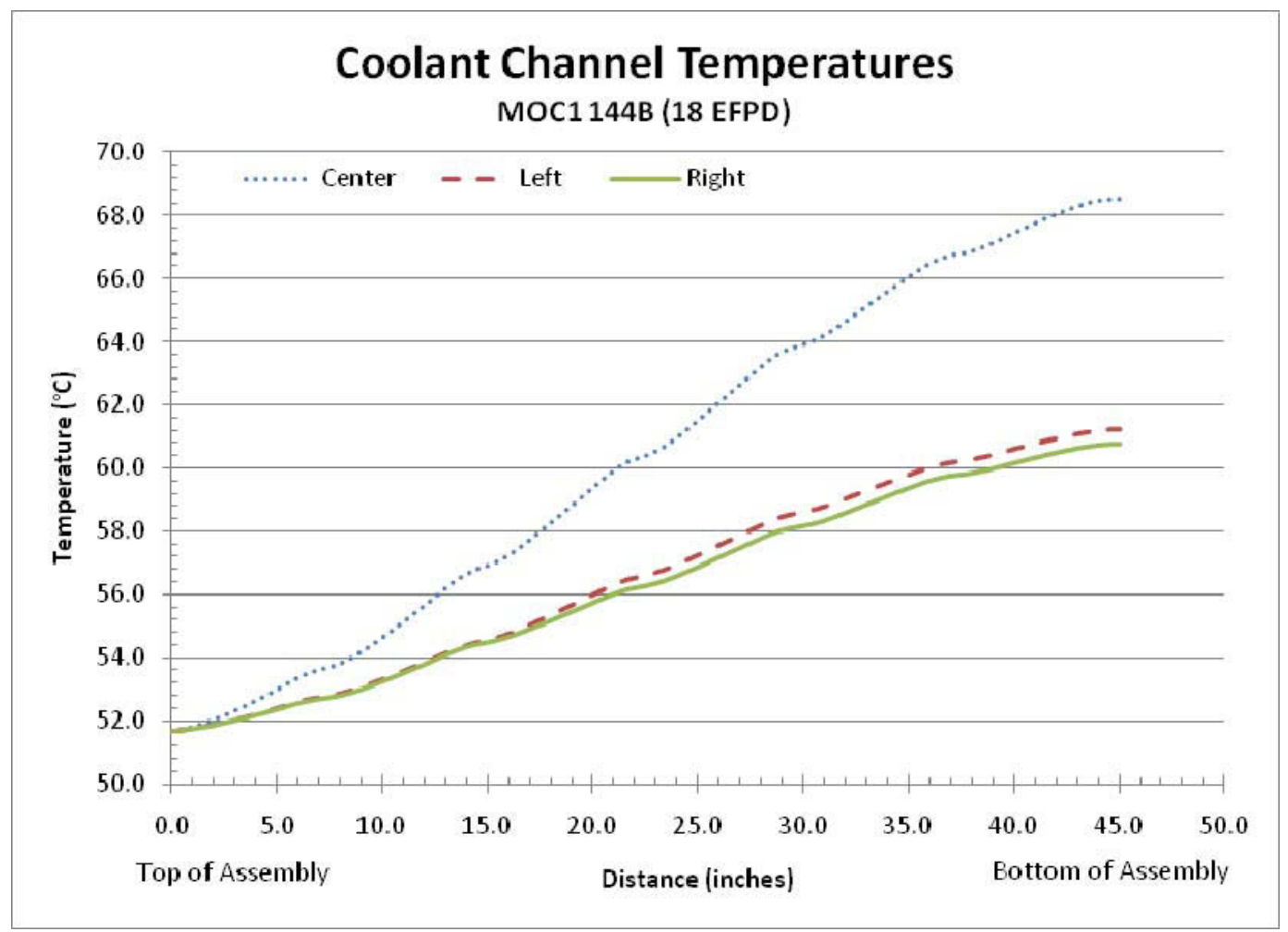

Figure 14: Coolant channel temperatures as a function of location along the AFIP-4 test assembly at MOC1 144B (18.0 EFPD).

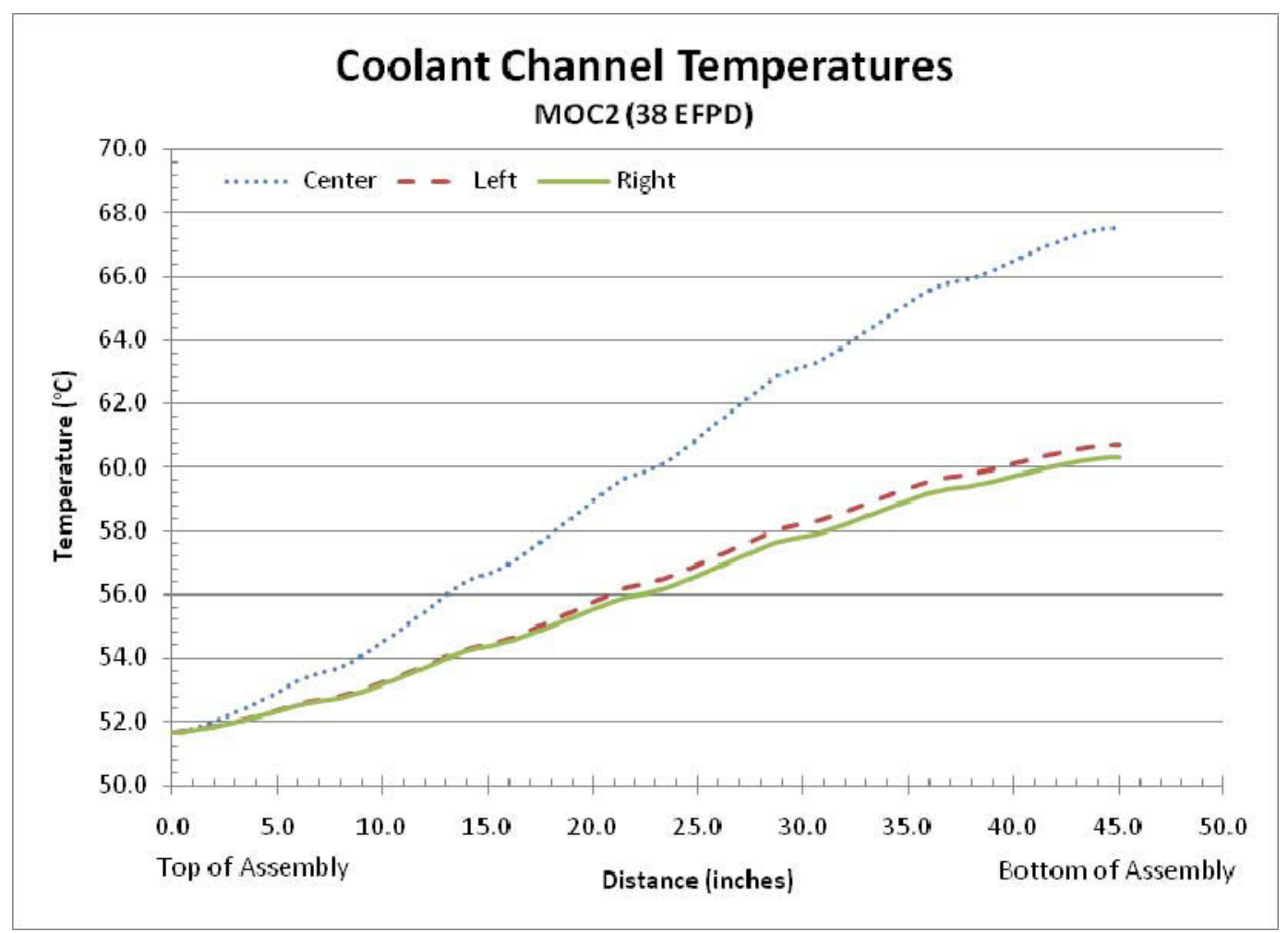

Figure 15: Coolant channel temperatures as a function of location along the AFIP-4 test assembly at MOC2 144B (38.0 EFPD). 


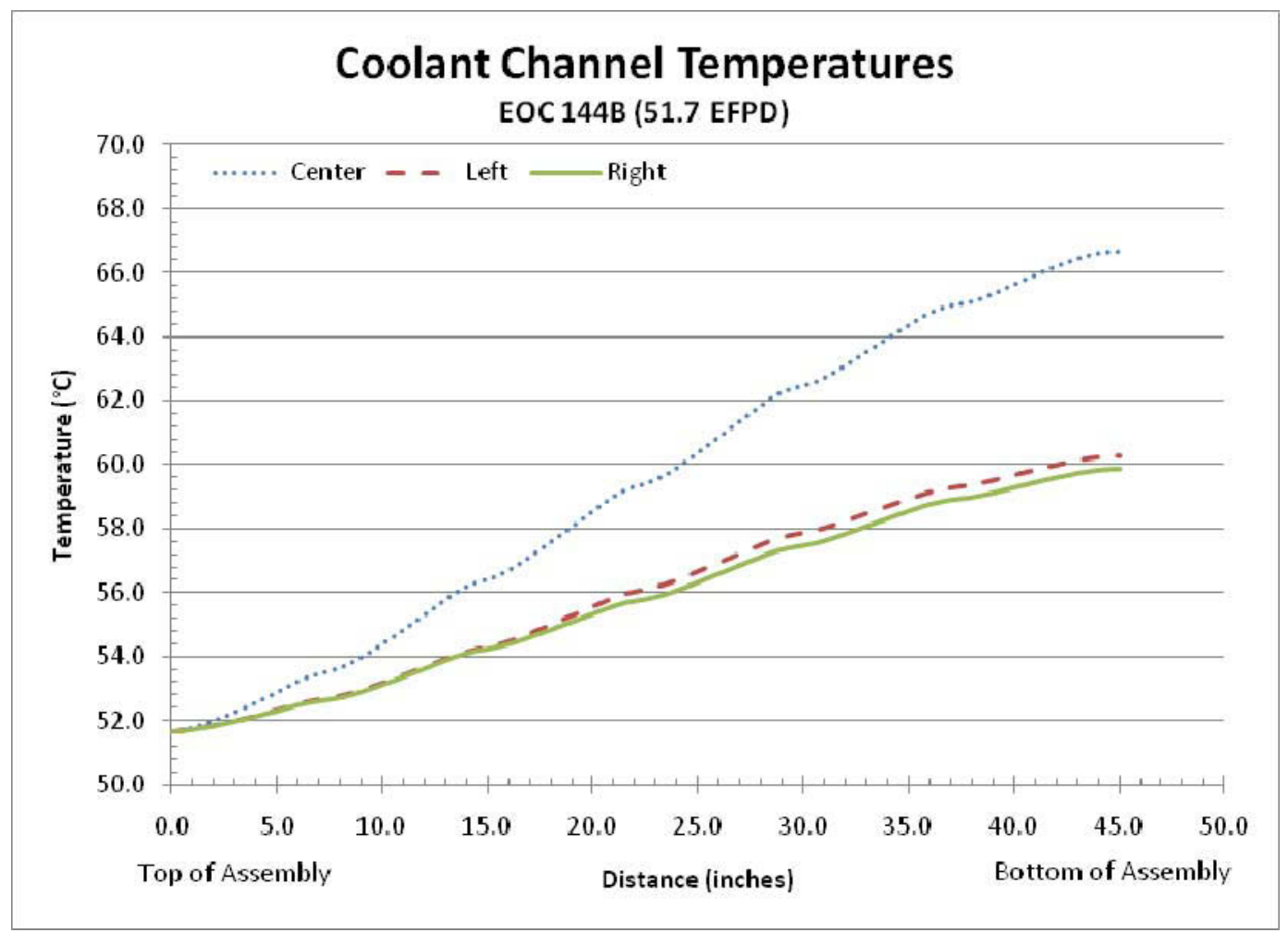

Figure 16: Coolant channel temperatures as a function of location along the AFIP-4 test assembly at EOC 144B (51.7 EFPD).

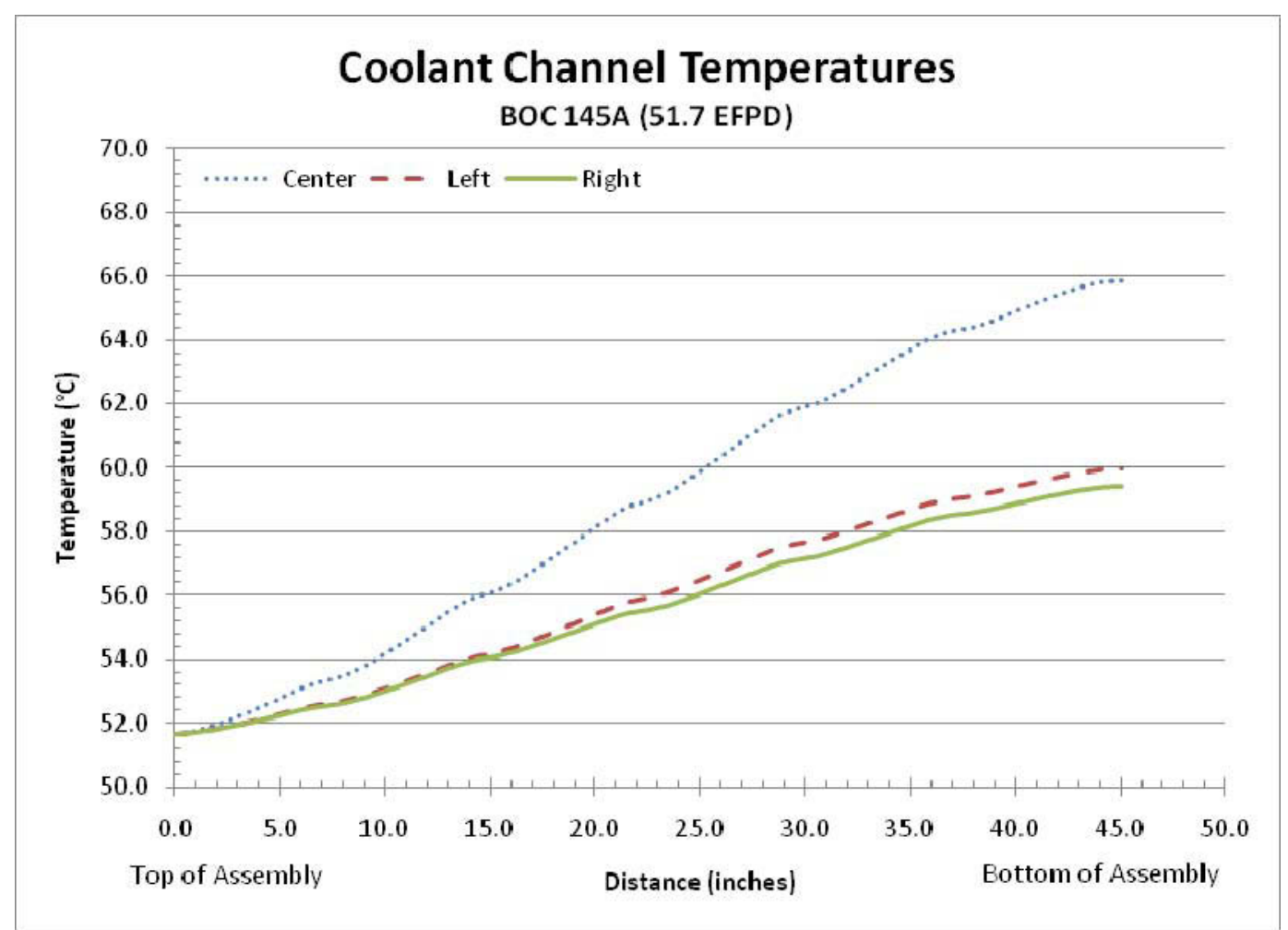

Figure 17: Coolant channel temperatures as a function of location along the AFIP-4 test assembly at BOC 145A (0 EFPD, cumulative 51.7 EFPD). 


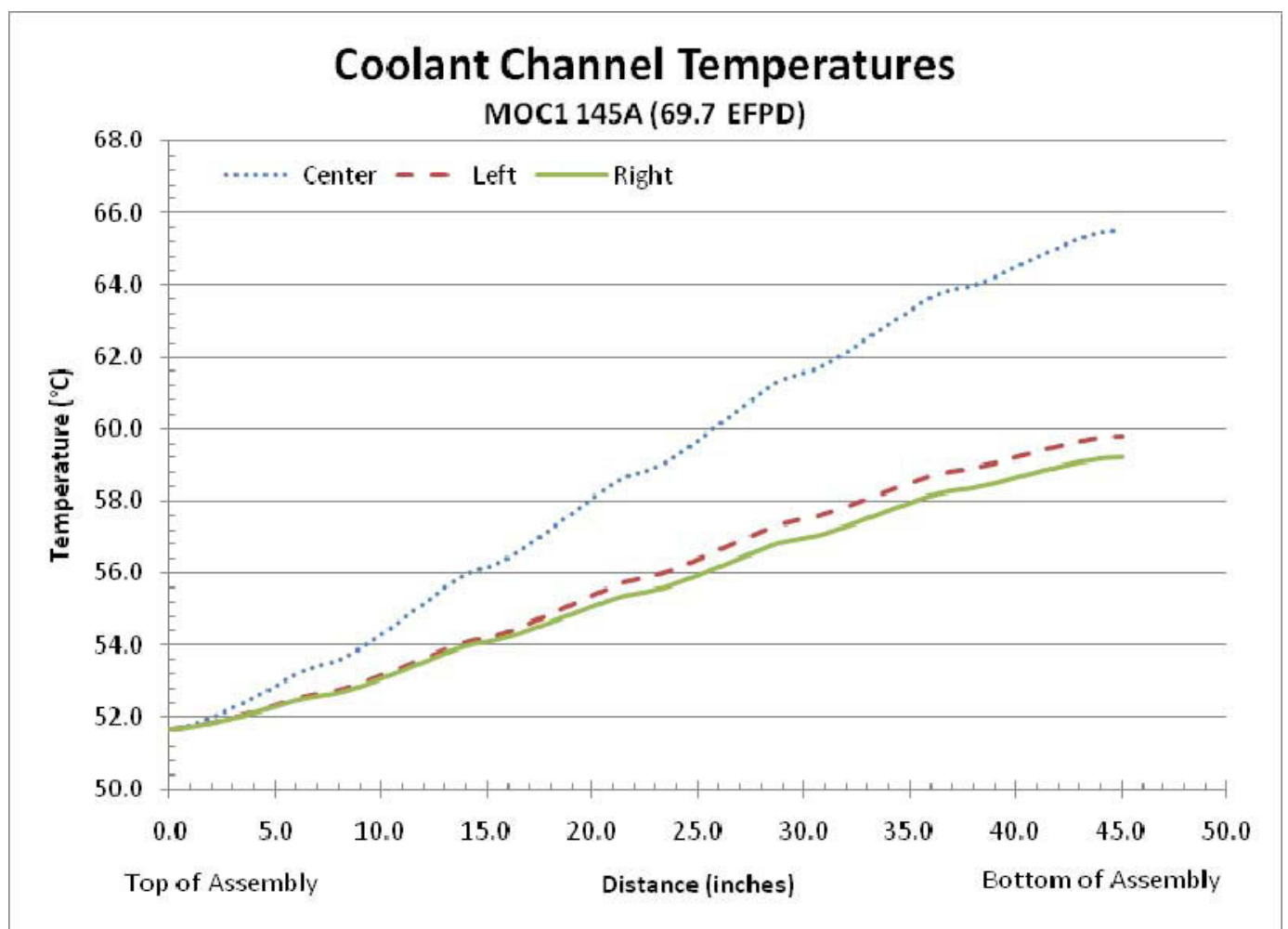

Figure 18: Coolant channel temperatures as a function of location along the AFIP-4 test assembly at MOC1 145A (18 EFPD, cumulative 69.7 EFPD).

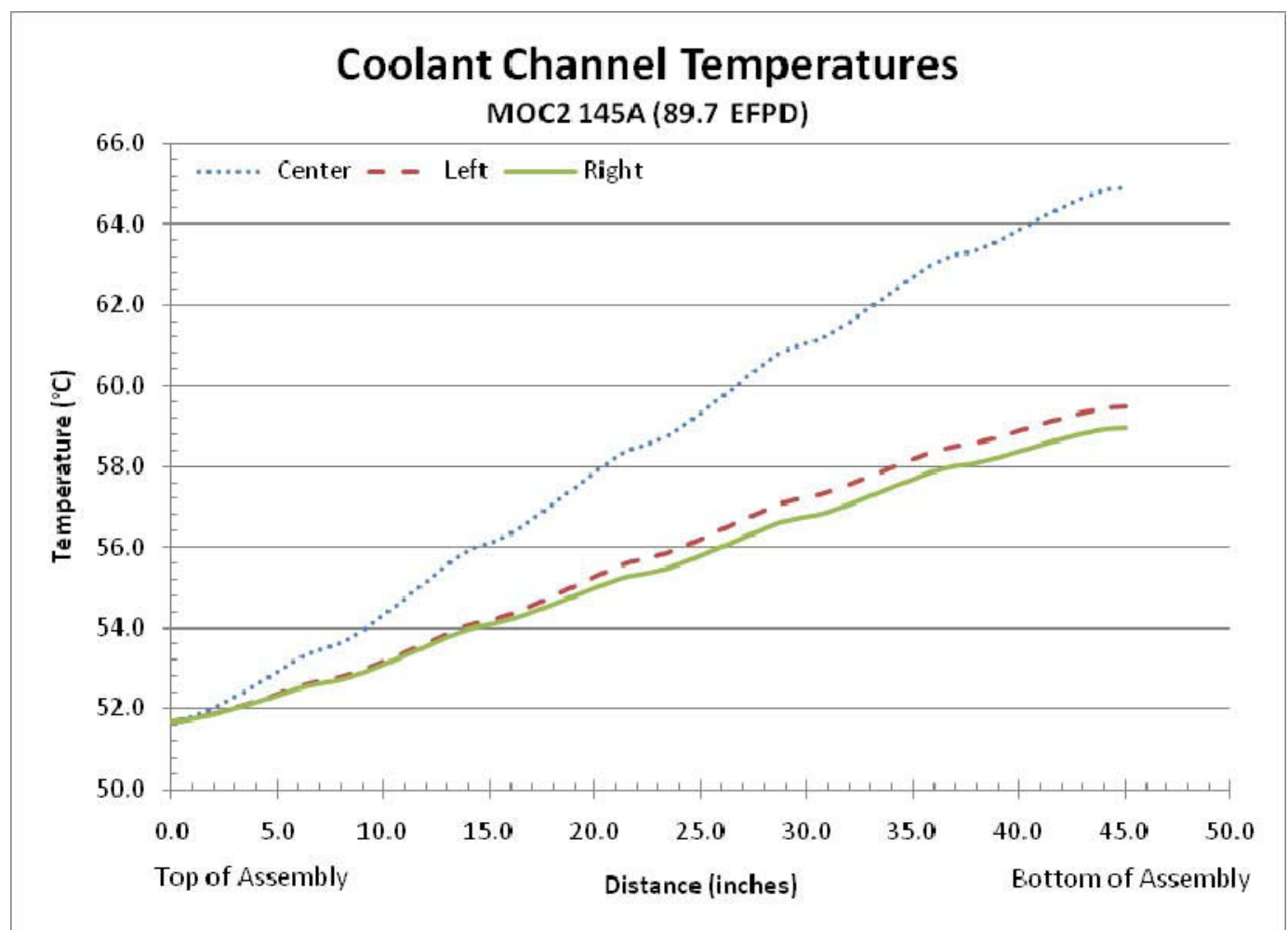

Figure 19: Coolant channel temperatures as a function of location along the AFIP-4 test assembly at MOC2 145A (38 EFPD, cumulative 89.7 EFPD). 


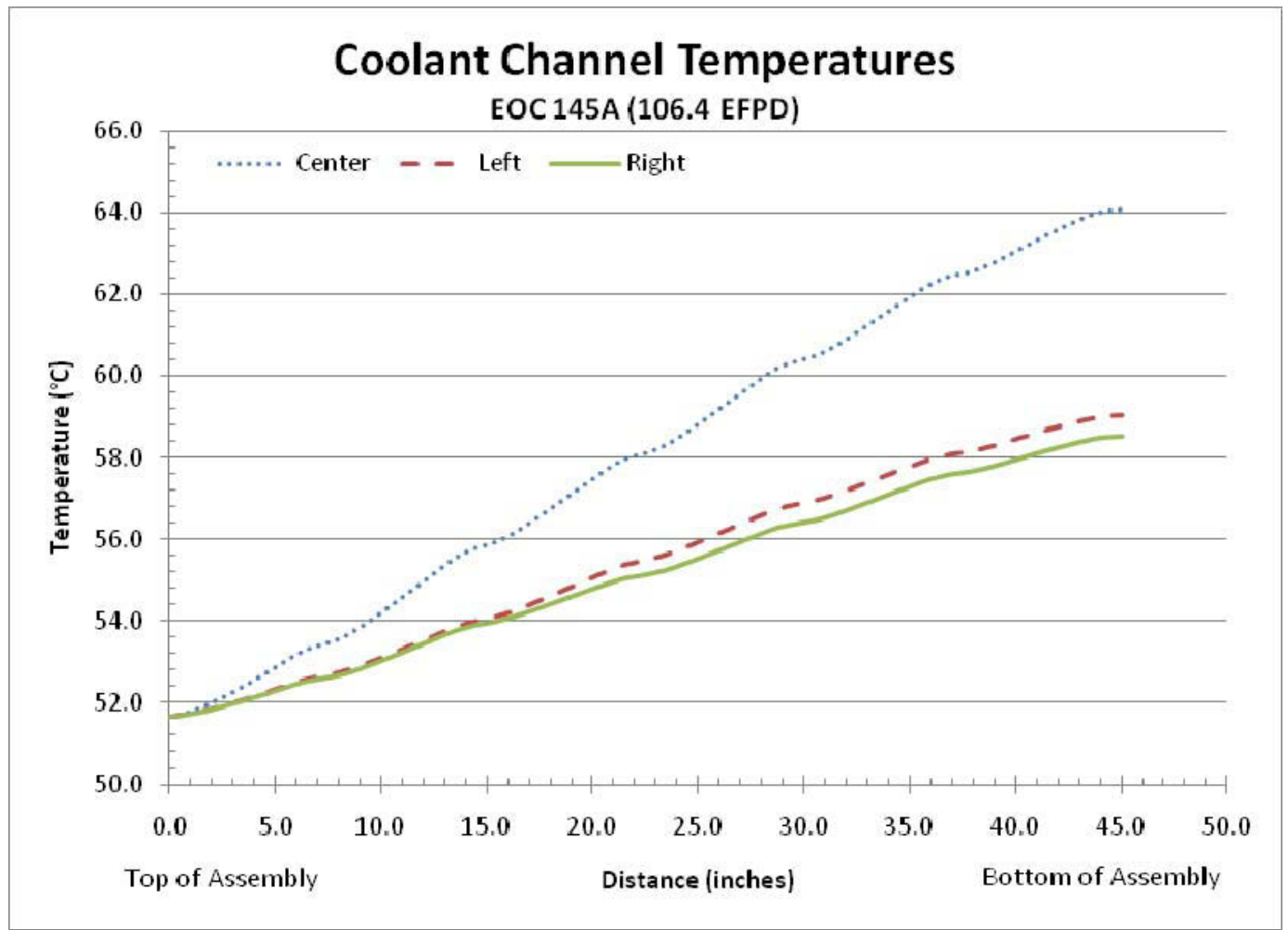

Figure 20: Coolant channel temperatures as a function of location along the AFIP-4 test assembly at EOC 145A (54.7 EFPD, cumulative 106.4 EFPD).

\subsection{Plate Surface Temperature}

The plate surface temperatures were analyzed at each time step for each side of the plate, with the east side of the plate facing the ram rod. Table 25 through Table 28 tabulate the $2 \mathrm{D}$ map of the temperatures for each side of the plates in position A and position B at EOC for cycle 144B. Table 29 - Table 32 tabulate the 2D map of the temperatures for each side of plates in position A and position B at EOC for cycle 145A. The plate surface temperatures for each time step are tabulated in Appendix B. 
Table 25: Temperature $\left({ }^{\circ} \mathrm{C}\right)$ map of the east side of position A at EOC 144B (51.7 EFPD).

\begin{tabular}{|c|c|c|c|c|c|c|c|c|c|c|c|}
\hline \multirow{2}{*}{$\begin{array}{c}\text { Plate } \\
\text { Location }\end{array}$} & \multirow{2}{*}{$\begin{array}{c}\text { Axial } \\
\text { Location (in) }\end{array}$} & \multicolumn{10}{|c|}{ Width of Plate (inches) } \\
\hline & & 0.00 & 0.08 & 0.16 & 0.25 & 0.50 & 1.00 & 1.50 & 2.00 & 2.08 & 2.16 \\
\hline \multirow{9}{*}{ A-1 } & $\mathbf{0 . 0}$ & 51.67 & 51.77 & 51.76 & 51.76 & 51.76 & 51.76 & 51.76 & 51.76 & 51.77 & 51.67 \\
\hline & 1.0 & 52.48 & 53.83 & 75.45 & 77.82 & 77.89 & 77.89 & 77.62 & 66.16 & 53.83 & 52.47 \\
\hline & 2.0 & 52.65 & 54.17 & 78.58 & 81.20 & 81.29 & 81.29 & 80.97 & 68.16 & 54.16 & 52.61 \\
\hline & 3.0 & 52.81 & 54.37 & 79.10 & 81.81 & 81.92 & 81.92 & 81.57 & 68.55 & 54.36 & 52.76 \\
\hline & 4.0 & 53.00 & 54.73 & 82.19 & 85.14 & 85.26 & 85.26 & 84.88 & 70.55 & 54.72 & 52.96 \\
\hline & 5.0 & 53.17 & 55.06 & 84.76 & 87.92 & 88.06 & 88.06 & 87.64 & 72.23 & 55.05 & 53.13 \\
\hline & 6.0 & 53.37 & 55.51 & 88.38 & 91.83 & 91.98 & 91.98 & 91.53 & 74.61 & 55.50 & 53.32 \\
\hline & 7.0 & 53.34 & 53.18 & 56.12 & 57.04 & 57.20 & 57.20 & 56.80 & 54.84 & 53.16 & 53.27 \\
\hline & 7.5 & 53.35 & 52.65 & 53.03 & 53.27 & 53.40 & 53.40 & 53.16 & 52.82 & 52.63 & 53.27 \\
\hline \multirow{9}{*}{ A-2 } & 7.5 & 53.35 & 52.65 & 53.03 & 53.27 & 53.40 & 53.40 & 53.16 & 52.82 & 52.63 & 53.27 \\
\hline & 8.0 & 53.50 & 53.37 & 56.75 & 57.80 & 57.97 & 57.97 & 57.53 & 55.29 & 53.35 & 53.42 \\
\hline & 9.0 & 53.90 & 56.36 & 94.34 & 98.23 & 98.41 & 98.41 & 97.89 & 78.59 & 56.35 & 53.86 \\
\hline & 10.0 & 54.07 & 56.62 & 95.67 & 99.68 & 99.91 & 99.91 & 99.32 & 79.52 & 56.61 & 54.02 \\
\hline & 11.0 & 54.28 & 56.98 & 97.77 & 102.11 & 102.37 & 102.37 & 101.69 & 80.95 & 56.97 & 54.21 \\
\hline & 12.0 & 54.46 & 57.23 & 98.99 & 103.54 & 103.82 & 103.82 & 103.10 & 81.81 & 57.22 & $\begin{array}{l}54.38 \\
\end{array}$ \\
\hline & 13.0 & 54.64 & 57.46 & 99.38 & 104.03 & 104.33 & 104.33 & 103.57 & 82.13 & 57.45 & 54.57 \\
\hline & 14.0 & 54.79 & 57.68 & 101.82 & 106.61 & 106.92 & 106.92 & 106.16 & 83.62 & 57.66 & 54.72 \\
\hline & 15.0 & 54.65 & 53.89 & 54.70 & 55.22 & 55.52 & 55.52 & 54.99 & 54.29 & 53.87 & 54.56 \\
\hline \multirow{9}{*}{ A-3 } & 15.0 & 54.65 & 53.89 & 54.70 & 55.22 & 55.52 & 55.52 & 54.99 & 54.29 & 53.87 & 54.56 \\
\hline & 16.0 & 55.12 & 57.69 & 97.92 & 102.36 & 102.68 & 102.68 & 101.89 & 81.31 & 57.66 & 55.00 \\
\hline & 17.0 & 55.30 & 57.88 & 98.02 & 102.50 & 102.85 & 102.85 & 102.02 & 81.45 & 57.87 & 55.23 \\
\hline & 18.0 & 55.45 & 58.06 & 98.19 & 102.72 & 103.09 & 103.09 & 102.23 & 81.62 & 58.05 & 55.40 \\
\hline & 19.0 & 55.64 & 58.28 & 98.45 & 103.04 & 103.43 & 103.43 & 102.53 & 81.86 & 58.26 & 55.57 \\
\hline & 20.0 & 55.80 & 58.53 & 99.52 & 104.20 & 104.61 & 104.61 & 103.68 & 82.58 & 58.51 & 55.70 \\
\hline & 21.0 & 55.98 & 58.83 & 100.91 & 105.71 & 106.14 & 106.14 & 105.17 & 83.51 & 58.81 & 55.88 \\
\hline & 22.0 & 55.88 & 55.94 & 60.29 & 61.86 & 62.33 & 62.33 & 61.37 & 58.40 & 55.92 & 55.79 \\
\hline & 22.5 & 55.85 & 55.24 & 56.27 & 56.99 & 57.43 & 57.43 & 56.66 & 55.77 & 55.22 & 55.79 \\
\hline \multirow{9}{*}{ A-4 } & 22.5 & 55.85 & 55.24 & 56.27 & 56.99 & 57.43 & 57.43 & 56.66 & 55.77 & 55.22 & 55.79 \\
\hline & 23.0 & 56.01 & 56.13 & 60.76 & 62.40 & 62.87 & 62.87 & 61.89 & 58.76 & 56.11 & 55.92 \\
\hline & 24.0 & 56.41 & 59.66 & 105.54 & 110.71 & 111.15 & 111.15 & 110.14 & 86.51 & 59.63 & 56.29 \\
\hline & 25.0 & 56.54 & 59.77 & 104.68 & 109.79 & 110.25 & 110.25 & 109.20 & 86.04 & 59.75 & 56.43 \\
\hline & 26.0 & 56.69 & 59.99 & 104.85 & 109.98 & 110.47 & 110.47 & 109.38 & 86.22 & 59.97 & 56.61 \\
\hline & 27.0 & 56.81 & 60.11 & 104.18 & 109.27 & 109.77 & 109.77 & 108.66 & 85.87 & 60.10 & 56.75 \\
\hline & 28.0 & 56.93 & 60.29 & 104.35 & 109.45 & 109.97 & 109.97 & 108.84 & 86.04 & 60.28 & 56.85 \\
\hline & 29.0 & 57.01 & 60.40 & 105.03 & 110.14 & 110.65 & 110.65 & 109.54 & 86.46 & 60.38 & 56.90 \\
\hline & 30.0 & 56.83 & 56.73 & 58.08 & 59.01 & 59.59 & 59.59 & 58.58 & 57.45 & 56.70 & 56.71 \\
\hline \multirow{9}{*}{ A-5 } & 30.0 & 56.83 & 56.73 & 58.08 & 59.01 & 59.59 & 59.59 & 58.58 & $\begin{array}{l}57.46 \\
\end{array}$ & 56.70 & 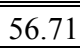 \\
\hline & 31.0 & 57.22 & 60.78 & 105.17 & 110.30 & 110.82 & 110.82 & 109.68 & 86.70 & 60.76 & 57.13 \\
\hline & 32.0 & 57.33 & 60.86 & 103.56 & 108.59 & 109.13 & 109.13 & 107.95 & 85.79 & 60.84 & 57.24 \\
\hline & 33.0 & 57.42 & 61.00 & 103.57 & 108.58 & 109.14 & 109.14 & 107.94 & 85.84 & 60.98 & 57.30 \\
\hline & 34.0 & 57.50 & 61.12 & 102.70 & 107.65 & 108.22 & 108.22 & 107.01 & 85.38 & 61.10 & 57.39 \\
\hline & 35.0 & 57.57 & 61.17 & 101.42 & 106.26 & 106.84 & 106.84 & 105.61 & 84.65 & 61.16 & 57.49 \\
\hline & 36.0 & 57.64 & 61.26 & 100.33 & 105.08 & 105.67 & 105.67 & 104.43 & 84.05 & 61.25 & 57.58 \\
\hline & 37.0 & 57.50 & 58.67 & 63.12 & 64.90 & 65.56 & 65.56 & 64.27 & 61.22 & 58.65 & 57.42 \\
\hline & 37.5 & 57.44 & 58.07 & 59.56 & 60.59 & 61.24 & 61.24 & 60.10 & 58.90 & 58.04 & 57.33 \\
\hline \multirow{9}{*}{ A-6 } & 37.5 & 57.44 & 58.07 & 59.56 & 60.59 & 61.24 & 61.24 & 60.10 & 58.90 & 58.04 & 57.33 \\
\hline & 38.0 & 57.53 & 58.69 & 62.78 & 64.47 & 65.12 & 65.12 & 63.86 & 61.04 & 58.67 & 57.42 \\
\hline & 39.0 & 57.75 & 61.34 & 97.08 & 101.51 & 102.10 & 102.10 & 100.88 & 82.20 & 61.32 & 57.65 \\
\hline & 40.0 & 57.77 & 61.27 & 94.43 & 98.61 & 99.21 & 99.21 & 97.98 & 80.62 & 61.25 & 57.69 \\
\hline & $\begin{array}{l}41.0 \\
\end{array}$ & 57.79 & 61.20 & 91.86 & 95.71 & 96.32 & 96.32 & 95.09 & 79.05 & 61.19 & 57.71 \\
\hline & 42.0 & 57.81 & 61.15 & 89.53 & 93.13 & 93.71 & 93.71 & 92.55 & 77.63 & 61.13 & 57.71 \\
\hline & 43.0 & 57.81 & 61.18 & 89.27 & 92.80 & 93.37 & 93.37 & 92.24 & 77.50 & 61.17 & 57.72 \\
\hline & 44.0 & 57.77 & 61.02 & 86.34 & 89.61 & 90.16 & 90.17 & 89.06 & 75.67 & 61.01 & 57.72 \\
\hline & 45.0 & 57.60 & 59.04 & 60.50 & 61.52 & 62.18 & 62.18 & 61.03 & 59.89 & 59.03 & 57.57 \\
\hline
\end{tabular}


Table 26: Temperature $\left({ }^{\circ} \mathrm{C}\right)$ map of the west side of position A at EOC 144B (51.7 EFPD).

\begin{tabular}{|c|c|c|c|c|c|c|c|c|c|c|c|}
\hline \multirow{2}{*}{$\begin{array}{c}\text { Plate } \\
\text { Location }\end{array}$} & \multirow{2}{*}{$\begin{array}{c}\text { Axial } \\
\text { Location (in) }\end{array}$} & \multicolumn{10}{|c|}{ Width of Plate (inches) } \\
\hline & & 0.00 & 0.08 & 0.16 & 0.25 & 0.50 & 1.00 & 1.50 & 2.00 & 2.08 & 2.16 \\
\hline \multirow{9}{*}{ A-1 } & 0.0 & 51.67 & 51.76 & 51.76 & 51.76 & 51.76 & 51.76 & 51.76 & 51.76 & 51.76 & 51.67 \\
\hline & 1.0 & 52.60 & 53.83 & 75.46 & 77.83 & 77.90 & 77.90 & 77.63 & 66.17 & 53.83 & 52.57 \\
\hline & 2.0 & 52.78 & 54.18 & 78.61 & 81.23 & 81.33 & 81.33 & 81.01 & 68.18 & 54.17 & 52.71 \\
\hline & 3.0 & 52.96 & 54.39 & 79.16 & 81.88 & 82.00 & 82.00 & 81.63 & 68.60 & 54.39 & 52.87 \\
\hline & 4.0 & 53.17 & 54.78 & 82.29 & 85.26 & 85.40 & 85.40 & 84.99 & 70.62 & 54.77 & 53.10 \\
\hline & 5.0 & 53.36 & 55.13 & 84.90 & 88.09 & 88.25 & 88.25 & 87.79 & 72.33 & 55.12 & 53.27 \\
\hline & 6.0 & 53.59 & 55.61 & 88.57 & 92.06 & 92.24 & 92.24 & 91.73 & 74.75 & 55.60 & 53.47 \\
\hline & 7.0 & 53.53 & 53.30 & 56.31 & 57.28 & 57.46 & 57.46 & 57.02 & 55.01 & 53.29 & 53.40 \\
\hline & 7.5 & 53.53 & 52.78 & 53.24 & 53.53 & 53.69 & 53.69 & 53.40 & 53.01 & 52.77 & 53.38 \\
\hline \multirow{9}{*}{ A-2 } & 7.5 & 53.53 & 52.78 & 53.24 & 53.53 & 53.69 & 53.69 & 53.40 & 53.01 & 52.77 & 53.38 \\
\hline & 8.0 & 53.70 & 53.52 & 56.98 & 58.07 & 58.27 & 58.27 & 57.78 & 55.48 & 53.51 & 53.55 \\
\hline & 9.0 & 54.16 & 56.53 & 94.63 & 98.58 & 98.81 & 98.81 & 98.22 & 78.83 & 56.52 & 54.10 \\
\hline & 10.0 & 54.36 & 56.82 & 96.01 & 100.15 & 100.44 & 100.44 & 99.73 & 79.79 & 56.81 & 54.26 \\
\hline & 11.0 & 54.59 & 57.22 & 98.16 & 102.67 & 102.99 & 102.99 & 102.21 & 81.27 & 57.21 & 54.44 \\
\hline & 12.0 & 54.78 & 57.51 & 99.50 & 104.17 & 104.53 & 104.53 & 103.69 & 82.17 & 57.50 & 54.61 \\
\hline & 13.0 & 54.98 & 57.78 & 99.98 & 104.74 & 105.13 & 105.13 & 104.23 & 82.54 & 57.77 & 54.83 \\
\hline & 14.0 & 55.15 & 58.04 & 102.49 & 107.41 & 107.81 & 107.81 & 106.89 & 84.08 & 58.03 & 54.98 \\
\hline & 15.0 & 54.96 & 54.29 & 55.29 & 55.92 & 56.30 & 56.30 & 55.63 & 54.80 & 54.28 & 54.76 \\
\hline \multirow{9}{*}{ A-3 } & 15.0 & 54.96 & 54.29 & 55.29 & 55.92 & 56.30 & 56.30 & 55.63 & 54.80 & 54.28 & 54.76 \\
\hline & 16.0 & 55.51 & 58.12 & 98.66 & 103.30 & 103.73 & 103.73 & 102.77 & 81.86 & 58.10 & 55.27 \\
\hline & 17.0 & 55.70 & 58.35 & 98.84 & 103.52 & 103.98 & 103.98 & 102.96 & 82.04 & 58.34 & 55.57 \\
\hline & 18.0 & 55.88 & 58.58 & 99.10 & 103.82 & 104.31 & 104.31 & 103.24 & 82.26 & 58.56 & 55.78 \\
\hline & 19.0 & 56.08 & 58.85 & 99.47 & 104.24 & 104.76 & 104.76 & 103.64 & 82.56 & 58.83 & 55.94 \\
\hline & 20.0 & 56.25 & 59.15 & 100.63 & 105.50 & 106.04 & 106.04 & 104.88 & 83.34 & 59.13 & 56.05 \\
\hline & 21.0 & 56.45 & 59.51 & 102.11 & 107.11 & 107.68 & 107.68 & 106.47 & 84.33 & 59.49 & 56.24 \\
\hline & 22.0 & 56.31 & 56.67 & 61.29 & 63.03 & 63.62 & 63.62 & 62.45 & 59.29 & 56.65 & 56.12 \\
\hline & 22.5 & 56.27 & 55.99 & 57.31 & 58.21 & 58.78 & 58.78 & 57.79 & 56.69 & 55.98 & 56.17 \\
\hline \multirow{9}{*}{ A-4 } & 22.5 & 56.27 & 55.99 & 57.31 & 58.21 & 58.78 & 58.78 & 57.79 & 56.69 & 55.98 & 56.17 \\
\hline & 23.0 & 56.45 & 56.90 & 61.80 & 63.62 & 64.22 & 64.22 & 63.02 & 59.69 & 56.89 & 56.26 \\
\hline & 24.0 & 56.92 & 60.46 & 106.92 & 112.30 & 112.91 & 112.91 & 111.62 & 87.46 & 60.44 & 56.68 \\
\hline & 25.0 & 57.06 & 60.63 & 106.13 & 111.46 & 112.10 & 112.10 & 110.76 & 87.05 & 60.60 & 56.83 \\
\hline & 26.0 & 57.23 & 60.91 & 106.39 & 111.76 & 112.42 & 112.42 & 111.04 & 87.29 & 60.88 & 57.07 \\
\hline & 27.0 & 57.36 & 61.09 & 105.81 & 111.15 & 111.83 & 111.83 & 110.41 & 87.01 & 61.06 & 57.24 \\
\hline & 28.0 & 57.49 & 61.32 & 106.06 & 111.42 & 112.13 & 112.13 & 110.67 & 87.24 & 61.30 & 57.33 \\
\hline & 29.0 & 57.57 & 61.49 & 106.83 & 112.20 & 112.92 & 112.92 & 111.46 & 87.72 & 61.47 & 57.34 \\
\hline & 30.0 & 57.33 & 57.88 & 59.59 & 60.76 & 61.51 & 61.51 & 60.20 & 58.81 & 57.86 & 57.10 \\
\hline \multirow{9}{*}{ A-5 } & 30.0 & 57.33 & 57.88 & 59.59 & 60.76 & 61.51 & 61.51 & 60.20 & 58.81 & 57.86 & 57.10 \\
\hline & 31.0 & 57.80 & 61.96 & 107.09 & 112.49 & 113.22 & 113.22 & 111.73 & 88.05 & 61.93 & 57.60 \\
\hline & 32.0 & 57.92 & 62.09 & 105.55 & 110.86 & 111.61 & 111.61 & 110.08 & 87.20 & 62.06 & 57.71 \\
\hline & 33.0 & 58.01 & 62.28 & 105.63 & 110.93 & 111.70 & 111.70 & 110.14 & 87.31 & 62.25 & 57.77 \\
\hline & 34.0 & 58.11 & 62.46 & 104.86 & 110.10 & 110.89 & 110.89 & 109.30 & 86.92 & 62.43 & 57.87 \\
\hline & 35.0 & 58.18 & 62.57 & 103.65 & 108.79 & 109.60 & 109.60 & 107.99 & 86.25 & 62.54 & 58.01 \\
\hline & 36.0 & 58.26 & 62.71 & 102.65 & 107.71 & 108.53 & 108.53 & 106.90 & 85.72 & 62.69 & 58.12 \\
\hline & 37.0 & 58.07 & 60.19 & 65.00 & 67.04 & 67.90 & 67.90 & 66.28 & 62.95 & 60.16 & 57.91 \\
\hline & 37.5 & 58.00 & 59.60 & 61.49 & 62.78 & 63.63 & 63.63 & 62.16 & 60.65 & 59.58 & 57.77 \\
\hline \multirow{9}{*}{ A-6 } & 37.5 & 58.00 & 59.60 & 61.49 & 62.78 & 63.63 & 63.63 & 62.16 & 60.65 & 59.58 & 57.77 \\
\hline & 38.0 & 58.10 & 60.25 & 64.70 & 66.65 & 67.49 & 67.49 & 65.91 & 62.80 & 60.22 & 57.88 \\
\hline & 39.0 & 58.37 & 62.92 & 99.58 & 104.33 & 105.15 & 105.15 & 103.53 & 84.01 & 62.89 & 58.15 \\
\hline & 40.0 & 58.40 & 62.89 & 96.98 & 101.51 & 102.35 & 102.35 & 100.72 & 82.49 & 62.87 & 58.20 \\
\hline & 41.0 & 58.41 & 62.88 & 94.42 & 98.71 & 99.55 & 99.55 & 97.92 & 80.99 & 62.85 & 58.22 \\
\hline & 42.0 & 58.42 & 62.88 & 92.09 & 96.18 & 97.03 & 97.03 & 95.40 & 79.64 & 62.84 & 58.21 \\
\hline & 43.0 & 58.43 & 62.95 & 91.89 & 95.91 & 96.75 & 96.75 & 95.14 & 79.54 & 62.92 & 58.22 \\
\hline & 44.0 & 58.38 & 62.84 & 88.97 & 92.71 & 93.55 & 93.55 & 91.97 & 77.79 & 62.81 & 58.25 \\
\hline & 45.0 & 58.17 & 60.89 & 62.74 & 64.03 & 64.89 & 64.89 & 63.40 & 61.95 & 60.87 & 58.09 \\
\hline
\end{tabular}


Table 27: Temperature $\left({ }^{\circ} \mathrm{C}\right)$ map of the east side of position B at EOC 144B (51.7 EFPD).

\begin{tabular}{|c|c|c|c|c|c|c|c|c|c|c|c|}
\hline \multirow{2}{*}{$\begin{array}{l}\text { Plate } \\
\text { Location }\end{array}$} & \multirow{2}{*}{$\begin{array}{c}\text { Axial } \\
\text { Location (in) }\end{array}$} & \multicolumn{10}{|c|}{ Width of Plate (inches) } \\
\hline & & 0.00 & 0.08 & 0.16 & 0.25 & 0.50 & 1.00 & 1.50 & 2.00 & 2.08 & 2.16 \\
\hline \multirow{9}{*}{ B-1 } & 0.0 & 51.67 & 51.79 & 51.76 & 51.76 & 51.76 & 51.76 & 51.76 & 51.76 & 51.79 & 51.67 \\
\hline & 1.0 & 52.94 & 53.86 & 75.02 & 77.35 & 77.42 & 77.42 & 77.15 & 65.90 & 53.84 & 52.84 \\
\hline & 2.0 & 53.16 & 54.21 & 78.09 & 80.67 & 80.76 & 80.76 & 80.45 & 67.85 & 54.16 & 52.91 \\
\hline & 3.0 & 53.37 & 54.44 & 78.64 & 81.32 & 81.43 & 81.43 & 81.07 & 68.27 & 54.38 & 53.01 \\
\hline & 4.0 & 53.63 & 54.84 & 81.79 & 84.72 & 84.86 & 84.86 & 84.45 & 70.31 & 54.78 & 53.29 \\
\hline & 5.0 & 53.86 & 55.24 & 84.88 & 88.07 & 88.23 & 88.23 & 87.77 & 72.33 & 55.17 & 53.45 \\
\hline & 6.0 & 54.13 & 55.69 & 88.06 & 91.50 & 91.69 & 91.69 & 91.18 & 74.43 & 55.60 & 53.64 \\
\hline & 7.0 & 54.10 & 53.43 & 56.28 & 57.23 & 57.41 & 57.41 & 56.97 & 54.99 & 53.34 & 53.59 \\
\hline & 7.5 & 54.12 & 52.93 & 53.26 & 53.53 & 53.69 & 53.69 & 53.41 & 53.02 & 52.83 & 53.58 \\
\hline \multirow{9}{*}{ B-2 } & 7.5 & $\begin{array}{l}54.12 \\
\end{array}$ & $\begin{array}{l}52.93 \\
\end{array}$ & 53.26 & 53.53 & 53.69 & 53.69 & 53.41 & 53.02 & 52.83 & 53.58 \\
\hline & 8.0 & 54.30 & 53.62 & 56.77 & 57.81 & 58.01 & 58.01 & 57.53 & 55.36 & 53.53 & 53.74 \\
\hline & 9.0 & 54.80 & 56.47 & 92.43 & 96.21 & 96.43 & 96.43 & 95.85 & 77.40 & 56.44 & 54.70 \\
\hline & 10.0 & 55.02 & 56.74 & 93.44 & 97.31 & 97.56 & 97.56 & 96.94 & 78.12 & 56.68 & 54.78 \\
\hline & 11.0 & 55.28 & 57.12 & 95.21 & 99.28 & 99.59 & 99.59 & 98.84 & 79.35 & 57.01 & 54.79 \\
\hline & 12.0 & 55.51 & 57.46 & 97.04 & 101.39 & 101.74 & 101.74 & 100.93 & 80.60 & 57.35 & 54.83 \\
\hline & 13.0 & 55.73 & 57.74 & 97.48 & 101.97 & 102.35 & 102.35 & 101.47 & 80.98 & 57.63 & 55.10 \\
\hline & 14.0 & 55.92 & 57.97 & 99.40 & 104.05 & 104.44 & 104.44 & 103.55 & 82.18 & 57.86 & 55.24 \\
\hline & 15.0 & 55.76 & 54.51 & 55.30 & 55.91 & 56.29 & 56.29 & 55.63 & 54.83 & 54.37 & 55.05 \\
\hline \multirow{9}{*}{ B-3 } & $\begin{array}{l}15.0 \\
\end{array}$ & 255.76 & $\begin{array}{c}54.51 \\
\end{array}$ & 255.30 & $\begin{array}{l}55.91 \\
\end{array}$ & $\begin{array}{l}56.29 \\
\end{array}$ & $\begin{array}{c}56.29 \\
\end{array}$ & $\begin{array}{l}55.63 \\
\end{array}$ & $\begin{array}{c}54.83 \\
\end{array}$ & $\begin{array}{c}54.37 \\
\end{array}$ & 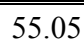 \\
\hline & 16.0 & 56.35 & 58.64 & 102.24 & 107.16 & 107.59 & 107.59 & 106.62 & 84.09 & 58.51 & 55.55 \\
\hline & 17.0 & 56.56 & 58.86 & 102.07 & 107.01 & 107.47 & 107.47 & 106.44 & 84.06 & 58.77 & 56.24 \\
\hline & 18.0 & 56.75 & 59.09 & 102.33 & 107.30 & 107.79 & 107.79 & 106.71 & 84.29 & 59.05 & 56.59 \\
\hline & 19.0 & 56.98 & 59.38 & 102.85 & 107.88 & 108.40 & 108.40 & 107.27 & 84.68 & 59.29 & 56.61 \\
\hline & 20.0 & 57.16 & 59.64 & 103.44 & 108.53 & 109.07 & 109.07 & 107.90 & 85.11 & 59.50 & 56.50 \\
\hline & 21.0 & 57.37 & 60.04 & 105.21 & 110.45 & 111.02 & 111.02 & 109.80 & 86.27 & 59.90 & 56.62 \\
\hline & 22.0 & 57.23 & 57.01 & 61.71 & 63.50 & 64.09 & 64.09 & 62.90 & 59.60 & 56.87 & 56.45 \\
\hline & 22.5 & 57.19 & 56.28 & 57.45 & 58.34 & 58.91 & 58.91 & 57.92 & 56.82 & 56.23 & 57.07 \\
\hline \multirow{9}{*}{ B-4 } & 22.5 & 57.19 & 56.28 & 57.45 & 58.34 & 58.91 & 58.91 & 57.92 & 56.82 & 56.23 & 57.07 \\
\hline & 23.0 & 57.38 & 57.17 & 61.85 & 63.64 & 64.24 & 64.23 & 63.04 & 59.75 & 57.03 & 56.58 \\
\hline & 24.0 & 57.85 & 60.67 & 106.18 & 111.49 & 112.09 & 112.09 & 110.81 & 87.05 & 60.51 & 56.94 \\
\hline & 25.0 & 57.99 & 60.81 & 105.13 & 110.37 & 111.00 & 111.00 & 109.67 & 86.48 & 60.66 & 57.29 \\
\hline & 26.0 & 58.15 & 61.11 & 105.56 & 110.85 & 111.51 & 111.51 & 110.13 & 86.83 & 61.00 & 57.81 \\
\hline & 27.0 & 58.28 & 61.33 & 105.45 & 110.74 & 111.42 & 111.42 & 110.01 & 86.84 & 61.27 & 58.09 \\
\hline & 28.0 & 58.41 & 61.58 & 105.72 & 111.04 & 111.75 & 111.74 & 110.29 & 87.08 & 61.47 & 58.03 \\
\hline & 29.0 & 58.48 & 61.74 & 106.31 & 111.63 & 112.34 & 112.34 & 110.89 & 87.45 & 61.59 & 57.78 \\
\hline & 30.0 & 58.23 & 58.19 & 59.73 & 60.88 & 61.62 & 61.62 & 60.32 & 58.95 & 58.03 & 57.40 \\
\hline \multirow{9}{*}{ B-5 } & 30.0 & $\begin{array}{l}58.23 \\
\end{array}$ & 58.19 & 59.73 & 60.88 & 61.62 & 61.62 & 60.32 & 58.95 & 58.03 & 57.40 \\
\hline & 31.0 & $\begin{array}{l}58.68 \\
\end{array}$ & 61.98 & 103.94 & 109.07 & 109.80 & 109.80 & 108.32 & 86.18 & 61.85 & 57.98 \\
\hline & 32.0 & 58.78 & 62.09 & 102.27 & 107.29 & 108.04 & 108.04 & 106.52 & 85.24 & 61.95 & 58.00 \\
\hline & 33.0 & 58.86 & 62.28 & 102.33 & 107.34 & 108.11 & 108.11 & 106.57 & 85.34 & 62.13 & 57.99 \\
\hline & 34.0 & $\begin{array}{l}58.93 \\
\end{array}$ & 62.46 & 101.49 & 106.44 & 107.22 & 107.22 & 105.65 & 84.91 & 62.30 & 58.23 \\
\hline & 35.0 & 58.99 & 62.56 & 100.32 & 105.17 & 105.97 & 105.97 & 104.37 & 84.27 & 62.46 & 58.61 \\
\hline & 36.0 & 59.04 & 62.79 & 100.34 & 105.18 & 106.00 & 106.00 & 104.38 & 84.36 & 62.73 & 58.76 \\
\hline & 37.0 & 58.83 & 60.43 & 64.90 & 66.87 & 67.72 & 67.72 & 66.12 & 62.94 & 60.33 & 58.53 \\
\hline & 37.5 & 58.75 & 59.89 & 61.62 & 62.89 & 63.72 & 63.72 & 62.26 & 60.79 & 59.75 & 58.00 \\
\hline \multirow{9}{*}{ B-6 } & 37.5 & 58.75 & $\begin{array}{l}59.89 \\
\end{array}$ & 61.62 & 62.89 & 63.72 & 63.72 & 62.26 & 60.79 & 59.75 & 58.00 \\
\hline & 38.0 & 58.84 & 60.52 & 64.74 & 66.63 & 67.47 & 67.46 & 65.90 & 62.88 & 60.37 & 58.20 \\
\hline & 39.0 & 59.07 & 63.10 & 98.55 & 103.19 & 104.00 & 104.00 & 102.40 & 83.44 & 62.96 & 58.37 \\
\hline & 40.0 & 59.07 & 63.07 & 95.99 & 100.41 & 101.24 & 101.24 & 99.62 & 81.94 & 62.94 & 58.38 \\
\hline & 41.0 & 59.05 & 63.04 & 93.30 & 97.46 & 98.30 & 98.30 & 96.68 & 80.37 & 62.92 & 58.43 \\
\hline & 42.0 & 59.03 & 63.04 & 91.01 & 94.98 & 95.82 & 95.82 & 94.21 & 79.03 & 62.90 & 58.33 \\
\hline & 43.0 & 59.00 & 63.11 & 90.84 & 94.74 & 95.58 & 95.58 & 93.98 & 78.96 & 62.99 & 58.45 \\
\hline & 44.0 & 58.92 & 63.02 & 88.27 & 91.91 & 92.74 & 92.74 & 91.17 & 77.42 & 62.94 & 58.65 \\
\hline & 45.0 & 58.70 & 61.14 & 62.90 & 64.17 & 65.01 & 65.01 & 63.54 & 62.12 & 61.09 & 58.57 \\
\hline
\end{tabular}


Table 28: Temperature $\left({ }^{\circ} \mathrm{C}\right)$ map of the west side of position B at EOC 144B (51.7 EFPD).

\begin{tabular}{|c|c|c|c|c|c|c|c|c|c|c|c|}
\hline \multirow{2}{*}{$\begin{array}{c}\text { Plate } \\
\text { Location }\end{array}$} & \multirow{2}{*}{$\begin{array}{c}\text { Axial } \\
\text { Location (in) }\end{array}$} & \multicolumn{10}{|c|}{ Width of Plate (inches) } \\
\hline & & 0.00 & 0.08 & 0.16 & 0.25 & 0.50 & 1.00 & 1.50 & 2.00 & 2.08 & 2.16 \\
\hline \multirow{9}{*}{ B-1 } & 0.0 & 51.67 & 51.78 & 51.76 & 51.76 & 51.76 & 51.76 & 51.76 & 51.76 & 51.78 & 51.67 \\
\hline & 1.0 & 53.25 & 53.84 & 75.01 & 77.33 & 77.40 & 77.40 & 77.13 & 65.89 & 53.82 & 53.13 \\
\hline & 2.0 & 53.50 & 54.18 & 78.06 & 80.63 & 80.72 & 80.72 & 80.41 & 67.83 & 54.14 & 53.07 \\
\hline & 3.0 & 53.74 & 54.39 & 78.58 & 81.25 & 81.35 & 81.35 & 81.01 & 68.23 & 54.35 & 53.03 \\
\hline & 4.0 & 54.03 & 54.76 & 81.70 & 84.61 & 84.73 & 84.73 & 84.35 & 70.25 & 54.72 & 53.35 \\
\hline & 5.0 & 54.29 & 55.14 & 84.76 & 87.91 & 88.05 & 88.05 & 87.63 & 72.24 & 55.10 & 53.47 \\
\hline & 6.0 & 54.58 & 55.56 & 87.89 & 91.29 & 91.45 & 91.45 & 90.99 & 74.31 & 55.50 & 53.67 \\
\hline & 7.0 & 54.61 & 53.28 & 56.10 & 57.01 & 57.16 & 57.16 & 56.77 & 54.84 & 53.22 & 53.71 \\
\hline & 7.5 & 54.65 & 52.77 & 53.07 & 53.29 & 53.42 & 53.42 & 53.19 & 52.86 & 52.70 & 53.72 \\
\hline \multirow{9}{*}{ B-2 } & 7.5 & 54.65 & 52.77 & 53.07 & 53.29 & 53.42 & 53.42 & 53.19 & 52.86 & 52.70 & 53.72 \\
\hline & 8.0 & 54.84 & 53.46 & 56.57 & 57.56 & 57.72 & 57.72 & 57.30 & 55.19 & 53.39 & 53.85 \\
\hline & 9.0 & 55.32 & 56.29 & 92.16 & 95.88 & 96.05 & 96.05 & 95.55 & 77.20 & 56.26 & 55.35 \\
\hline & 10.0 & 55.56 & 56.53 & 93.13 & 96.93 & 97.13 & 97.12 & 96.59 & 77.89 & 56.49 & 55.29 \\
\hline & 11.0 & 55.84 & 56.87 & 94.84 & 98.78 & 99.00 & 99.00 & 98.42 & 79.07 & 56.79 & 54.99 \\
\hline & 12.0 & 56.08 & 57.17 & 96.62 & 100.79 & 101.06 & 101.06 & 100.37 & 80.28 & 57.09 & 54.79 \\
\hline & 13.0 & 56.31 & 57.41 & 97.01 & 101.28 & 101.57 & 101.57 & 100.84 & 80.61 & 57.33 & 55.08 \\
\hline & 14.0 & 56.51 & 57.61 & 98.79 & 103.28 & 103.57 & 103.57 & 102.85 & 81.77 & 57.52 & 55.18 \\
\hline & 15.0 & 56.42 & 54.10 & 54.76 & 55.25 & 55.53 & 55.53 & 55.02 & 54.37 & 54.00 & 55.15 \\
\hline \multirow{9}{*}{ B-3 } & 15.0 & 56.42 & 54.10 & 54.76 & 55.25 & 55.53 & 55.53 & 55.02 & 54.37 & 54.00 & 55.15 \\
\hline & 16.0 & 56.98 & 58.22 & 101.51 & 106.28 & 106.60 & 106.60 & 105.81 & 83.61 & 58.11 & 55.57 \\
\hline & 17.0 & 57.18 & 58.41 & 101.29 & 106.07 & 106.41 & 106.40 & 105.57 & 83.55 & 58.34 & 56.81 \\
\hline & 18.0 & 57.38 & 58.61 & 101.50 & 106.30 & 106.65 & 106.65 & 105.79 & 83.73 & 58.56 & 57.35 \\
\hline & 19.0 & 57.60 & 58.85 & 101.94 & 106.79 & 107.17 & 107.16 & 106.26 & 84.07 & 58.78 & 57.16 \\
\hline & 20.0 & 57.78 & 59.08 & 102.47 & 107.36 & 107.76 & 107.76 & 106.82 & 84.45 & 58.96 & 56.67 \\
\hline & 21.0 & 57.98 & 59.42 & 104.17 & 109.20 & 109.61 & 109.61 & 108.65 & 85.57 & 59.31 & 56.61 \\
\hline & 22.0 & 57.88 & 56.34 & 60.84 & 62.45 & 62.91 & 62.90 & 61.94 & 58.84 & 56.24 & 56.35 \\
\hline & 22.5 & 57.85 & 55.60 & 56.55 & 57.25 & 57.68 & 57.68 & 56.91 & 56.03 & 55.54 & 57.97 \\
\hline \multirow{9}{*}{ B-4 } & 22.5 & 57.85 & 55.60 & 56.55 & 57.25 & 57.68 & 57.68 & 56.91 & 56.03 & 55.54 & 57.97 \\
\hline & 23.0 & 58.02 & 56.48 & 60.94 & 62.55 & 63.01 & 63.01 & 62.04 & 58.96 & 56.37 & 56.47 \\
\hline & 24.0 & 58.43 & 59.95 & 104.98 & 110.06 & 110.49 & 110.49 & 109.49 & 86.23 & 59.82 & 56.76 \\
\hline & 25.0 & 58.55 & 60.06 & 103.87 & 108.87 & 109.32 & 109.32 & 108.29 & 85.61 & 59.93 & 57.39 \\
\hline & 26.0 & 58.69 & 60.31 & 104.22 & 109.26 & 109.73 & 109.72 & 108.66 & 85.91 & 60.21 & 58.36 \\
\hline & 27.0 & 58.82 & 60.48 & 104.04 & 109.06 & 109.54 & 109.54 & 108.45 & 85.86 & 60.41 & 58.77 \\
\hline & 28.0 & 58.92 & 60.68 & 104.23 & 109.27 & 109.77 & 109.77 & 108.66 & 86.04 & 60.58 & 58.48 \\
\hline & 29.0 & 58.97 & 60.79 & 104.74 & 109.77 & 110.27 & 110.26 & 109.17 & 86.36 & 60.65 & 57.79 \\
\hline & 30.0 & 58.75 & 57.18 & 58.42 & 59.31 & 59.87 & 59.87 & 58.87 & 57.78 & 57.05 & 57.25 \\
\hline \multirow{9}{*}{ B-5 } & $\begin{array}{c}30.0 \\
\end{array}$ & 58.75 & 57.18 & 58.42 & 59.31 & 259.87 & 59.87 & 58.88 & 57.78 & 57.05 & \begin{tabular}{c|}
57.25 \\
\end{tabular} \\
\hline & 31.0 & 59.07 & 60.82 & 102.22 & 107.01 & 107.51 & 107.50 & 106.42 & 84.91 & 60.70 & 57.48 \\
\hline & 32.0 & 59.19 & 61.03 & 100.51 & 105.23 & 105.75 & 105.75 & 104.61 & 84.01 & 60.90 & 57.68 \\
\hline & 33.0 & 59.24 & 61.17 & 100.51 & 105.21 & 105.74 & 105.74 & 104.58 & 84.05 & 61.03 & 57.61 \\
\hline & 34.0 & 59.27 & 61.29 & 99.58 & 104.20 & 104.75 & 104.74 & 103.57 & 83.55 & 61.15 & 58.10 \\
\hline & 35.0 & 59.30 & 61.35 & 98.33 & 102.84 & 103.40 & 103.39 & 102.21 & 82.85 & 61.25 & 58.85 \\
\hline & 36.0 & 59.31 & 61.53 & 98.27 & 102.77 & 103.33 & 103.33 & 102.13 & 82.89 & 61.44 & 59.03 \\
\hline & 37.0 & 59.10 & 59.11 & 63.23 & 64.91 & 65.55 & 65.54 & 64.29 & 61.41 & 59.01 & 58.85 \\
\hline & 37.5 & 59.02 & 58.55 & 59.90 & 60.88 & 61.50 & 61.49 & 60.39 & 59.24 & 58.41 & 57.67 \\
\hline \multirow{9}{*}{ B-6 } & 37.5 & 59.02 & 58.55 & 59.90 & 60.88 & 61.50 & 61.49 & 60.39 & 59.24 & 58.41 & 57.67 \\
\hline & 38.0 & 59.07 & 59.16 & 63.03 & 64.64 & 65.26 & 65.26 & 64.04 & 61.32 & 59.03 & 58.01 \\
\hline & 39.0 & 59.23 & 61.72 & 96.32 & 100.60 & 101.16 & 101.15 & 99.98 & 81.83 & 61.59 & 57.97 \\
\hline & 40.0 & 59.19 & 61.66 & 93.72 & 97.75 & 98.32 & 98.31 & 97.12 & 80.28 & 61.53 & 57.85 \\
\hline & 41.0 & 59.13 & 61.58 & 91.03 & 94.71 & 95.28 & 95.28 & 94.09 & 78.63 & 61.45 & 57.98 \\
\hline & 42.0 & 59.07 & 61.53 & 88.74 & 92.19 & 92.72 & 92.72 & 91.62 & 77.24 & 61.40 & 57.76 \\
\hline & 43.0 & 59.00 & 61.58 & 88.50 & 91.90 & 92.43 & 92.42 & 91.33 & 77.12 & 61.44 & 58.08 \\
\hline & 44.0 & 58.87 & 61.44 & 85.90 & 89.06 & 89.59 & 89.58 & 88.51 & 75.52 & 61.34 & 58.51 \\
\hline & 45.0 & 58.66 & 59.53 & 60.90 & 61.86 & 62.48 & 62.48 & 61.37 & 60.27 & 59.45 & 58.52 \\
\hline
\end{tabular}


Table 29: Temperature $\left({ }^{\circ} \mathrm{C}\right)$ map of the east side of position A at EOC 145A (106.4 cumulative).

\begin{tabular}{|c|c|c|c|c|c|c|c|c|c|c|c|}
\hline \multirow{2}{*}{$\begin{array}{c}\text { Plate } \\
\text { Location }\end{array}$} & \multirow{2}{*}{$\begin{array}{c}\text { Axial } \\
\text { Location (in) }\end{array}$} & \multicolumn{10}{|c|}{ Width of Plate (inches) } \\
\hline & & 0.00 & 0.08 & 0.16 & 0.25 & 0.50 & 1.00 & 1.50 & 2.00 & 2.08 & 2.16 \\
\hline \multirow{9}{*}{ A-1 } & 0.0 & 51.67 & 51.77 & 51.76 & 51.77 & 51.77 & 51.77 & 51.77 & 51.76 & 51.77 & 51.67 \\
\hline & 1.0 & 52.51 & 53.86 & 75.65 & 78.04 & 78.11 & 78.11 & 78.04 & 75.65 & 53.85 & 52.49 \\
\hline & 2.0 & 52.67 & 54.16 & 78.40 & 81.01 & 81.10 & 81.10 & 81.01 & 78.40 & 54.16 & 52.64 \\
\hline & 3.0 & 52.83 & 54.34 & 78.84 & 81.53 & 81.63 & 81.63 & 81.53 & 78.84 & 54.34 & 52.79 \\
\hline & 4.0 & 53.02 & 54.63 & 81.05 & 83.91 & 84.03 & 84.03 & 83.91 & 81.05 & 54.63 & 52.99 \\
\hline & 5.0 & 53.19 & 54.93 & 83.35 & 86.40 & 86.54 & 86.54 & 86.40 & 83.35 & 54.92 & 53.15 \\
\hline & 6.0 & 53.39 & 55.22 & 85.21 & 88.41 & 88.56 & 88.56 & 88.41 & 85.21 & 55.21 & 53.33 \\
\hline & 7.0 & 53.38 & 53.11 & 55.77 & 56.62 & 56.77 & 56.77 & 56.62 & 55.77 & 53.09 & 53.30 \\
\hline & 7.5 & 53.39 & 52.63 & 52.98 & 53.20 & 53.33 & 53.33 & 53.20 & 52.98 & 52.61 & 53.31 \\
\hline \multirow{9}{*}{ A-2 } & 7.5 & 53.39 & 52.63 & 52.98 & 53.20 & 53.33 & 53.33 & 53.20 & 52.98 & 52.61 & 53.31 \\
\hline & 8.0 & 53.53 & 53.28 & 56.29 & 57.24 & 57.40 & 57.40 & 57.24 & 56.29 & 53.26 & 53.45 \\
\hline & 9.0 & 53.90 & 55.95 & 90.14 & 93.72 & 93.89 & 93.89 & 93.72 & 90.14 & 55.94 & 53.87 \\
\hline & 10.0 & 54.07 & 56.15 & 90.82 & 94.46 & 94.66 & 94.66 & 94.46 & 90.82 & 56.14 & 54.02 \\
\hline & 11.0 & 54.26 & 56.33 & 91.14 & 94.81 & 95.02 & 95.02 & 94.81 & 91.14 & 56.31 & 54.19 \\
\hline & 12.0 & 54.43 & 56.53 & 91.97 & 95.71 & 95.93 & 95.93 & 95.71 & 91.97 & 56.51 & 54.35 \\
\hline & 13.0 & 54.60 & 56.70 & 92.18 & 95.95 & 96.18 & 96.18 & 95.95 & 92.18 & 56.68 & 54.53 \\
\hline & 14.0 & 54.74 & 56.77 & 92.66 & 96.43 & 96.67 & 96.67 & 96.43 & 92.66 & 56.75 & 54.67 \\
\hline & 15.0 & 54.66 & 53.74 & 54.38 & 54.83 & 55.09 & 55.09 & 54.83 & 54.38 & 53.71 & 54.56 \\
\hline \multirow{9}{*}{ A-3 } & 15.0 & 54.66 & 53.74 & 54.38 & 54.83 & 55.09 & 55.09 & 54.83 & 54.38 & 53.71 & 54.56 \\
\hline & 16.0 & 55.05 & 56.68 & 88.32 & 91.79 & 92.05 & 92.05 & 91.79 & 88.32 & 56.66 & 54.93 \\
\hline & 17.0 & 55.21 & 56.86 & 88.65 & 92.15 & 92.42 & 92.42 & 92.15 & 88.65 & 56.85 & 55.14 \\
\hline & 18.0 & 55.36 & 57.02 & 88.81 & 92.33 & 92.61 & 92.61 & 92.33 & 88.81 & 57.01 & 55.31 \\
\hline & 19.0 & 55.53 & 57.20 & 89.09 & 92.64 & 92.93 & 92.93 & 92.64 & 89.09 & 57.18 & 55.46 \\
\hline & 20.0 & 55.67 & 57.32 & 88.95 & 92.49 & 92.79 & 92.79 & 92.49 & 88.95 & 57.30 & 55.56 \\
\hline & 21.0 & 55.82 & 57.47 & 88.94 & 92.48 & 92.79 & 92.79 & 92.48 & 88.94 & 57.45 & 55.72 \\
\hline & 22.0 & 55.77 & 55.34 & 58.52 & 59.74 & 60.13 & 60.13 & 59.74 & 58.52 & 55.32 & 55.67 \\
\hline & 22.5 & 55.75 & 54.84 & 55.59 & 56.17 & 56.53 & 56.53 & 56.17 & 55.59 & 54.82 & 55.70 \\
\hline \multirow{9}{*}{ A-4 } & 22.5 & 55.75 & 54.84 & 55.59 & 56.17 & 56.53 & 56.53 & 56.17 & 55.59 & 54.82 & 55.70 \\
\hline & 23.0 & 55.88 & 55.51 & 58.95 & 60.24 & 60.62 & 60.62 & 60.24 & 58.94 & 55.49 & 55.79 \\
\hline & 24.0 & 56.20 & 58.19 & 93.08 & 96.92 & 97.26 & 97.26 & 96.92 & 93.08 & 58.16 & 56.09 \\
\hline & 25.0 & 56.32 & 58.36 & 93.32 & 97.20 & 97.57 & 97.57 & 97.20 & 93.32 & 58.33 & 56.21 \\
\hline & 26.0 & 56.45 & 58.53 & 93.45 & 97.34 & 97.75 & 97.75 & 97.34 & 93.44 & 58.51 & 56.38 \\
\hline & 27.0 & 56.56 & 58.69 & 93.75 & 97.71 & 98.12 & 98.12 & 97.71 & 93.75 & 58.68 & 56.50 \\
\hline & 28.0 & 56.66 & 58.84 & 93.88 & 97.86 & 98.29 & 98.29 & 97.86 & 93.88 & 58.82 & 56.58 \\
\hline & 29.0 & 56.72 & 58.87 & 93.63 & 97.54 & 97.97 & 97.97 & 97.54 & 93.63 & 58.84 & 56.61 \\
\hline & 30.0 & 56.58 & 56.01 & 56.99 & 57.72 & 58.17 & 58.17 & 57.72 & 56.99 & 55.98 & 56.47 \\
\hline \multirow{9}{*}{ A-5 } & 30.0 & 56.58 & 56.01 & 56.99 & 57.72 & 58.17 & 58.17 & 57.72 & 56.99 & 55.98 & 56.47 \\
\hline & 31.0 & 56.89 & 59.20 & 94.15 & 98.15 & 98.58 & 98.58 & 98.15 & 94.15 & 59.18 & 56.80 \\
\hline & 32.0 & 56.98 & 59.37 & 94.27 & 98.31 & 98.75 & 98.75 & 98.31 & 94.27 & 59.35 & 56.89 \\
\hline & 33.0 & 57.05 & 59.51 & 94.40 & 98.47 & 98.93 & 98.93 & 98.47 & 94.40 & 59.49 & 56.94 \\
\hline & 34.0 & 57.12 & 59.66 & 94.35 & 98.42 & 98.89 & 98.89 & 98.42 & 94.35 & 59.64 & 57.02 \\
\hline & 35.0 & 57.18 & 59.75 & 93.89 & 97.91 & 98.39 & 98.39 & 97.91 & 93.88 & 59.74 & 57.10 \\
\hline & 36.0 & 57.23 & 59.87 & 93.61 & 97.59 & 98.08 & 98.08 & 97.59 & 93.60 & 59.86 & 57.17 \\
\hline & 37.0 & 57.09 & 57.62 & 61.30 & 62.79 & 63.32 & 63.32 & 62.78 & 61.30 & 57.60 & 57.02 \\
\hline & 37.5 & 57.03 & 57.09 & 58.22 & 59.04 & 59.55 & 59.55 & 59.03 & 58.21 & 57.06 & 56.93 \\
\hline \multirow{9}{*}{ A-6 } & $\begin{array}{l}37.5 \\
\end{array}$ & 57.03 & 57.09 & 58.22 & $\begin{array}{l}59.04 \\
\end{array}$ & 59.56 & 59.55 & 59.03 & 58.22 & 57.06 & 56.93 \\
\hline & 38.0 & 57.11 & 57.64 & 61.09 & 62.50 & 63.03 & 63.03 & 62.50 & 61.09 & 57.62 & 57.01 \\
\hline & 39.0 & 57.29 & 59.99 & 91.64 & 95.36 & 95.83 & 95.83 & 95.36 & 91.64 & 59.97 & 57.20 \\
\hline & 40.0 & 57.30 & 60.00 & 90.30 & 93.91 & 94.36 & 94.36 & 93.91 & 90.30 & 59.98 & 57.23 \\
\hline & 41.0 & 57.31 & 59.99 & 88.70 & 92.18 & 92.64 & 92.64 & 92.18 & 88.70 & 59.98 & 57.24 \\
\hline & 42.0 & 57.31 & 59.95 & 86.75 & 90.07 & 90.54 & 90.54 & 90.07 & 86.75 & 59.93 & 57.22 \\
\hline & 43.0 & 57.30 & 59.99 & 86.57 & 89.85 & 90.31 & 90.31 & 89.85 & 86.57 & 59.97 & 57.22 \\
\hline & 44.0 & 57.25 & 59.87 & 84.52 & 87.60 & 88.06 & 88.06 & 87.60 & 84.52 & 59.86 & 57.20 \\
\hline & 45.0 & 57.07 & 57.91 & 59.10 & 59.94 & 60.48 & 60.48 & 59.94 & 59.10 & 57.90 & 57.04 \\
\hline
\end{tabular}


Table 30: Temperature $\left({ }^{\circ} \mathrm{C}\right)$ map of the west side of position A at EOC 145A (106.4 cumulative).

\begin{tabular}{|c|c|c|c|c|c|c|c|c|c|c|c|}
\hline \multirow{2}{*}{$\begin{array}{c}\text { Plate } \\
\text { Location }\end{array}$} & \multirow{2}{*}{$\begin{array}{c}\text { Axial } \\
\text { Location (in) }\end{array}$} & \multicolumn{10}{|c|}{ Width of Plate (inches) } \\
\hline & & 0.00 & 0.08 & 0.16 & 0.25 & 0.50 & 1.00 & 1.50 & 2.00 & 2.08 & 2.16 \\
\hline \multirow{9}{*}{ A-1 } & 0.0 & 51.67 & 51.77 & 51.76 & 51.77 & 51.77 & 51.77 & 51.77 & 51.76 & 51.77 & 51.67 \\
\hline & 1.0 & 52.63 & 53.85 & 75.66 & 78.05 & 78.12 & 78.12 & 78.05 & 75.66 & 53.85 & 52.59 \\
\hline & 2.0 & 52.81 & 54.17 & 78.43 & 81.05 & 81.14 & 81.14 & 81.05 & 78.43 & 54.17 & 52.74 \\
\hline & 3.0 & 52.99 & 54.37 & 78.90 & 81.60 & 81.71 & 81.71 & 81.60 & 78.90 & 54.36 & 52.90 \\
\hline & 4.0 & 53.20 & 54.68 & 81.15 & 84.03 & 84.17 & 84.17 & 84.03 & 81.15 & 54.67 & 53.12 \\
\hline & 5.0 & 53.39 & 55.00 & 83.49 & 86.57 & 86.73 & 86.73 & 86.57 & 83.49 & 54.99 & 53.30 \\
\hline & 6.0 & 53.60 & 55.32 & 85.39 & 88.64 & 88.81 & 88.81 & 88.64 & 85.39 & 55.31 & 53.48 \\
\hline & 7.0 & 53.57 & 53.23 & 55.96 & 56.85 & 57.02 & 57.02 & 56.85 & 55.96 & 53.22 & 53.43 \\
\hline & 7.5 & 53.57 & 52.76 & 53.19 & 53.45 & 53.61 & 53.61 & 53.45 & 53.19 & 52.75 & 53.42 \\
\hline \multirow{9}{*}{ A-2 } & 7.5 & 53.57 & 52.76 & 53.19 & 53.45 & 53.61 & 53.61 & 53.45 & 53.19 & 52.75 & 53.42 \\
\hline & 8.0 & 53.73 & 53.42 & 56.51 & 57.50 & 57.69 & 57.69 & 57.50 & 56.50 & 53.41 & 53.57 \\
\hline & 9.0 & 54.17 & 56.11 & 90.43 & 94.06 & 94.28 & 94.28 & 94.06 & 90.43 & 56.10 & 54.10 \\
\hline & 10.0 & 54.35 & 56.33 & 91.15 & 94.86 & 95.09 & 95.09 & 94.86 & 91.15 & 56.32 & 54.25 \\
\hline & 11.0 & 54.56 & 56.55 & 91.52 & 95.27 & 95.53 & 95.53 & 95.27 & 91.52 & 56.54 & 54.41 \\
\hline & 12.0 & 54.75 & 56.78 & 92.40 & 96.22 & 96.51 & 96.51 & 96.22 & 92.40 & 56.77 & 54.57 \\
\hline & 13.0 & 54.93 & 56.98 & 92.67 & 96.53 & 96.83 & 96.83 & 96.52 & 92.67 & 56.97 & 54.78 \\
\hline & 14.0 & 55.08 & 57.09 & 93.21 & 97.07 & 97.39 & 97.39 & 97.07 & 93.21 & 57.08 & 54.92 \\
\hline & 15.0 & 54.96 & 54.09 & 54.90 & 55.44 & 55.78 & 55.78 & 55.44 & 54.90 & 54.08 & 54.76 \\
\hline \multirow{9}{*}{ A-3 } & 15.0 & 54.96 & 54.09 & 54.90 & 55.44 & 55.78 & 55.78 & 55.44 & 54.90 & 54.08 & 54.76 \\
\hline & 16.0 & 55.42 & 57.06 & 88.96 & 92.54 & 92.88 & 92.88 & 92.54 & 88.96 & 57.04 & 55.18 \\
\hline & 17.0 & 55.60 & 57.27 & 89.33 & 92.95 & 93.31 & 93.31 & 92.95 & 89.33 & 57.26 & 55.46 \\
\hline & 18.0 & 55.76 & 57.46 & 89.54 & 93.19 & 93.56 & 93.56 & 93.19 & 89.54 & 57.45 & 55.66 \\
\hline & 19.0 & 55.94 & 57.69 & 89.89 & 93.57 & 93.96 & 93.96 & 93.56 & 89.88 & 57.67 & 55.80 \\
\hline & 20.0 & 56.09 & 57.85 & 89.80 & 93.49 & 93.90 & 93.90 & 93.48 & 89.80 & 57.83 & 55.88 \\
\hline & 21.0 & 56.25 & 58.04 & 89.86 & 93.55 & 93.98 & 93.98 & 93.55 & 89.86 & 58.02 & 56.05 \\
\hline & 22.0 & 56.17 & 55.95 & 59.35 & 60.71 & 61.20 & 61.20 & 60.71 & 59.35 & 55.94 & 55.98 \\
\hline & 22.5 & 56.15 & 55.46 & 56.45 & 57.17 & 57.63 & 57.63 & 57.17 & 56.44 & 55.45 & 56.05 \\
\hline \multirow{9}{*}{ A-4 } & 22.5 & 56.15 & 55.46 & 56.45 & 57.17 & 57.63 & 57.63 & 57.17 & 56.44 & 55.45 & 56.05 \\
\hline & 23.0 & 56.30 & 56.15 & 59.81 & 61.24 & 61.73 & 61.73 & 61.24 & 59.80 & 56.13 & 56.10 \\
\hline & 24.0 & 56.67 & 58.86 & 94.10 & 98.24 & 98.74 & 98.74 & 98.24 & 94.10 & 58.83 & 56.43 \\
\hline & 25.0 & 56.80 & 59.06 & 94.40 & 98.60 & 99.12 & 99.12 & 98.59 & 94.39 & 59.04 & 56.58 \\
\hline & 26.0 & 56.94 & 59.28 & 94.58 & 98.83 & 99.38 & 99.38 & 98.83 & 94.57 & 59.26 & 56.79 \\
\hline & 27.0 & 57.05 & 59.49 & 94.94 & 99.26 & 99.83 & 99.83 & 99.26 & 94.94 & 59.47 & 56.93 \\
\hline & 28.0 & 57.16 & 59.68 & 95.14 & 99.49 & 100.08 & 100.07 & 99.49 & 95.14 & 59.65 & 57.00 \\
\hline & 29.0 & 57.22 & 59.75 & 94.96 & 99.25 & 99.84 & 99.84 & 99.25 & 94.95 & 59.72 & 57.00 \\
\hline & 30.0 & 57.04 & 56.94 & 58.19 & 59.10 & 59.69 & 59.69 & 59.10 & 58.19 & 56.91 & 56.81 \\
\hline \multirow{9}{*}{ A-5 } & 30.0 & 57.04 & 56.94 & 58.19 & 59.10 & 59.69 & 59.69 & 59.10 & 58.19 & 56.91 & 56.81 \\
\hline & 31.0 & 57.41 & 60.15 & 95.59 & 99.95 & 100.54 & 100.54 & 99.94 & 95.59 & 60.13 & 57.22 \\
\hline & 32.0 & 57.51 & 60.35 & 95.77 & 100.16 & 100.77 & 100.77 & 100.15 & 95.77 & 60.33 & 57.30 \\
\hline & 33.0 & 57.58 & 60.54 & 95.98 & 100.38 & 101.01 & 101.01 & 100.38 & 95.97 & 60.51 & 57.35 \\
\hline & 34.0 & 57.66 & 60.73 & 96.00 & 100.40 & 101.05 & 101.05 & 100.40 & 95.99 & 60.71 & 57.43 \\
\hline & 35.0 & 57.71 & 60.87 & 95.59 & 99.96 & 100.62 & 100.62 & 99.95 & 95.58 & 60.84 & 57.55 \\
\hline & 36.0 & 57.77 & 61.03 & 95.37 & 99.72 & 100.39 & 100.39 & 99.72 & 95.37 & 61.01 & 57.63 \\
\hline & 37.0 & 57.59 & 58.82 & 62.80 & 64.48 & 65.17 & 65.17 & 64.48 & 62.79 & 58.80 & 57.45 \\
\hline & 37.5 & 57.52 & 58.31 & 59.75 & 60.77 & 61.44 & 61.44 & 60.77 & 59.74 & 58.29 & 57.31 \\
\hline \multirow{9}{*}{ A-6 } & 37.5 & 57.52 & 58.31 & 59.75 & 60.77 & 61.44 & 61.44 & 60.77 & 59.74 & 58.29 & 257.31 \\
\hline & 38.0 & 57.61 & 58.88 & 62.61 & 64.23 & 64.90 & 64.90 & 64.22 & 62.61 & 58.85 & 57.41 \\
\hline & 39.0 & 57.83 & 61.25 & 93.49 & 97.63 & 98.30 & 98.29 & 97.62 & 93.49 & 61.23 & 57.63 \\
\hline & 40.0 & 57.84 & 61.30 & 92.17 & 96.19 & 96.87 & 96.87 & 96.19 & 92.16 & 61.27 & 57.66 \\
\hline & 41.0 & 57.85 & 61.33 & 90.63 & 94.48 & 95.17 & 95.16 & 94.48 & 90.63 & 61.31 & 57.67 \\
\hline & 42.0 & 57.84 & 61.33 & 88.75 & 92.39 & 93.08 & 93.08 & 92.38 & 88.75 & 61.30 & 57.64 \\
\hline & 43.0 & 57.83 & 61.41 & 88.62 & 92.21 & 92.91 & 92.91 & 92.21 & 88.61 & 61.38 & 57.65 \\
\hline & 44.0 & 57.77 & 61.33 & 86.65 & 89.99 & 90.68 & 90.68 & 89.98 & 86.64 & 61.31 & 57.66 \\
\hline & 45.0 & 57.56 & 59.40 & 60.91 & 61.96 & 62.66 & 62.66 & 61.96 & 60.90 & 59.38 & 57.49 \\
\hline
\end{tabular}


Table 31: Temperature $\left({ }^{\circ} \mathrm{C}\right)$ map of the east side of position B at EOC 145A (106.4 cumulative).

\begin{tabular}{|c|c|c|c|c|c|c|c|c|c|c|c|}
\hline \multirow{2}{*}{$\begin{array}{c}\text { Plate } \\
\text { Location }\end{array}$} & \multirow{2}{*}{$\begin{array}{c}\text { Axial } \\
\text { Location (in) }\end{array}$} & \multicolumn{10}{|c|}{ Width of Plate (inches) } \\
\hline & & 0.00 & 0.08 & 0.16 & 0.25 & 0.50 & 1.00 & 1.50 & 2.00 & 2.08 & 2.16 \\
\hline \multirow{9}{*}{ B-1 } & 0.0 & 51.67 & 51.79 & 51.76 & 51.77 & 51.77 & 51.77 & 51.77 & 51.76 & 51.79 & 51.67 \\
\hline & 1.0 & 52.98 & 53.91 & 75.40 & 77.77 & 77.84 & 77.84 & 77.77 & 75.40 & 53.88 & 52.87 \\
\hline & 2.0 & 53.20 & 54.24 & 78.29 & 80.89 & 80.99 & 80.99 & 80.89 & 78.29 & 54.19 & 52.95 \\
\hline & 3.0 & 53.42 & 54.45 & 78.76 & 81.44 & 81.55 & 81.55 & 81.44 & 78.76 & 54.39 & 53.04 \\
\hline & 4.0 & 53.67 & 54.76 & 80.84 & 83.70 & 83.83 & 83.83 & 83.70 & 80.84 & 54.70 & 53.32 \\
\hline & 5.0 & 53.90 & 55.07 & 82.98 & 86.01 & 86.17 & 86.17 & 86.01 & 82.98 & 55.00 & 53.48 \\
\hline & 6.0 & 54.16 & 55.44 & 85.41 & 88.64 & 88.82 & 88.82 & 88.64 & 85.40 & 55.36 & 53.66 \\
\hline & 7.0 & 54.15 & 53.38 & 55.98 & 56.86 & 57.04 & 57.04 & 56.86 & 55.97 & 53.28 & 53.63 \\
\hline & 7.5 & 54.17 & 52.91 & 53.21 & 53.46 & 53.62 & 53.62 & 53.46 & 53.20 & 52.82 & 53.62 \\
\hline \multirow{9}{*}{ B-2 } & 7.5 & 54.17 & 52.91 & 53.21 & 53.46 & 53.62 & 53.62 & 53.46 & 53.20 & 52.82 & 53.62 \\
\hline & 8.0 & 54.35 & 53.53 & 56.28 & 57.21 & 57.40 & 57.40 & 57.21 & 56.27 & 53.43 & 53.78 \\
\hline & 9.0 & 54.82 & 56.04 & 87.90 & 91.34 & 91.55 & 91.55 & 91.34 & 87.90 & 56.01 & 54.72 \\
\hline & 10.0 & 55.03 & 56.28 & 88.71 & 92.23 & 92.46 & 92.46 & 92.23 & 88.71 & 56.21 & 54.79 \\
\hline & 11.0 & 55.28 & 56.55 & 89.60 & 93.20 & 93.46 & 93.46 & 93.20 & 89.60 & 56.44 & 54.78 \\
\hline & 12.0 & 55.49 & 56.77 & 90.14 & 93.79 & 94.07 & 94.07 & 93.79 & 90.14 & 56.65 & 54.81 \\
\hline & 13.0 & 55.70 & 56.97 & 90.39 & 94.07 & 94.37 & 94.37 & 94.07 & 90.38 & 56.86 & 55.07 \\
\hline & 14.0 & 55.88 & 57.09 & 90.91 & 94.60 & 94.91 & 94.91 & 94.60 & 90.90 & 56.98 & 55.19 \\
\hline & 15.0 & 55.78 & 54.31 & 54.93 & 55.45 & 55.78 & 55.78 & 55.45 & 54.92 & 54.18 & 55.06 \\
\hline \multirow{9}{*}{ B-3 } & 15.0 & 55.78 & 54.31 & 54.93 & 55.45 & 55.78 & 55.78 & 55.45 & 54.92 & 54.18 & 55.06 \\
\hline & 16.0 & 56.28 & 57.54 & 91.76 & 95.54 & 95.87 & 95.87 & 95.54 & 91.75 & 57.40 & 55.47 \\
\hline & 17.0 & 56.48 & 57.78 & 92.39 & 96.22 & 96.58 & 96.58 & 96.22 & 92.39 & 57.69 & 56.15 \\
\hline & 18.0 & 56.66 & 57.98 & 92.62 & 96.48 & 96.89 & 96.89 & 96.48 & 92.61 & 57.93 & 56.49 \\
\hline & 19.0 & 56.86 & 58.21 & 92.88 & 96.79 & 97.23 & 97.23 & 96.79 & 92.87 & 58.11 & 56.50 \\
\hline & 20.0 & 57.02 & 58.39 & 92.96 & 96.90 & 97.36 & 97.36 & 96.90 & 92.95 & 58.25 & 56.36 \\
\hline & 21.0 & 57.19 & 58.59 & 93.01 & 96.98 & 97.46 & 97.46 & 96.98 & 93.00 & 58.45 & 56.45 \\
\hline & 22.0 & 57.11 & 56.30 & 59.79 & 61.20 & 61.69 & 61.69 & 61.20 & 59.78 & 56.16 & 56.33 \\
\hline & 22.5 & 57.09 & 55.76 & 56.59 & 57.31 & 57.77 & 57.77 & 57.30 & 56.58 & 55.71 & 56.97 \\
\hline \multirow{9}{*}{ B-4 } & 22.5 & 57.09 & 55.76 & 56.59 & 57.31 & 57.77 & 57.77 & 57.30 & 56.58 & 55.71 & 56.97 \\
\hline & 23.0 & 57.24 & 56.44 & 59.91 & 61.33 & 61.82 & 61.81 & 61.33 & 59.90 & 56.30 & 56.45 \\
\hline & 24.0 & 57.62 & 59.12 & 93.88 & 97.97 & 98.47 & 98.46 & 97.96 & 93.87 & 58.96 & 56.73 \\
\hline & 25.0 & 57.75 & 59.30 & 93.90 & 98.02 & 98.54 & 98.54 & 98.02 & 93.89 & 59.15 & 57.06 \\
\hline & 26.0 & 57.88 & 59.54 & 94.24 & 98.44 & 98.98 & 98.98 & 98.43 & 94.23 & 59.44 & 57.55 \\
\hline & 27.0 & 58.00 & 59.72 & 94.27 & 98.48 & 99.05 & 99.05 & 98.48 & 94.26 & 59.67 & 57.80 \\
\hline & 28.0 & 58.10 & 59.92 & 94.46 & 98.72 & 99.30 & 99.30 & 98.71 & 94.44 & 59.81 & 57.73 \\
\hline & 29.0 & 58.15 & 60.02 & 94.66 & 98.91 & 99.50 & 99.49 & 98.91 & 94.65 & 59.87 & 57.48 \\
\hline & 30.0 & 57.95 & 57.26 & 58.35 & 59.24 & 59.82 & 59.82 & 59.23 & 58.33 & 57.10 & 57.15 \\
\hline \multirow{9}{*}{ B-5 } & 30.0 & 57.95 & 57.26 & 58.35 & 59.24 & 59.82 & 59.82 & 59.23 & 58.33 & 57.10 & 57.15 \\
\hline & 31.0 & 58.30 & 60.25 & 93.28 & 97.37 & 97.95 & 97.95 & 97.36 & 93.26 & 60.12 & 57.63 \\
\hline & 32.0 & 58.39 & 60.44 & 93.22 & 97.33 & 97.93 & 97.93 & 97.32 & 93.21 & 60.30 & 57.63 \\
\hline & 33.0 & 58.44 & 60.61 & 93.38 & 97.52 & 98.14 & 98.14 & 97.51 & 93.37 & 60.46 & 57.61 \\
\hline & 34.0 & 58.49 & 60.80 & 93.27 & 97.41 & 98.05 & 98.05 & 97.41 & 93.26 & 60.65 & 57.83 \\
\hline & 35.0 & 58.53 & 60.96 & 93.21 & 97.34 & 97.99 & 97.99 & 97.34 & 93.19 & 60.86 & 58.18 \\
\hline & 36.0 & 58.56 & 61.10 & 92.72 & 96.81 & 97.47 & 97.47 & 96.80 & 92.70 & 61.03 & 58.29 \\
\hline & \begin{tabular}{|l|l}
37.0 \\
\end{tabular} & 58.36 & 59.08 & 62.69 & 64.29 & 64.97 & 64.96 & 64.28 & 62.67 & 58.98 & 58.09 \\
\hline & 37.5 & 58.28 & 58.61 & 59.90 & 60.89 & 61.55 & 61.55 & 60.88 & 59.87 & 58.47 & 57.58 \\
\hline \multirow{9}{*}{ B-6 } & 37.5 & 58.28 & 58.61 & 59.90 & 60.89 & 61.55 & 61.55 & 60.88 & 59.88 & 58.47 & 57.58 \\
\hline & 38.0 & 58.35 & 59.16 & 62.66 & 64.22 & 64.88 & 64.88 & 64.21 & 62.63 & 59.02 & 57.76 \\
\hline & 39.0 & 58.54 & 61.44 & 92.38 & 96.39 & 97.05 & 97.05 & 96.38 & 92.36 & 61.31 & 57.89 \\
\hline & 40.0 & 58.52 & 61.47 & 90.99 & 94.85 & 95.52 & 95.51 & 94.84 & 90.97 & 61.35 & 57.88 \\
\hline & 41.0 & 58.49 & 61.50 & 89.42 & 93.09 & 93.77 & 93.77 & 93.09 & 89.40 & 61.38 & 57.92 \\
\hline & 42.0 & 58.45 & 61.52 & 87.93 & 91.44 & 92.13 & 92.12 & 91.43 & 87.91 & 61.40 & 57.81 \\
\hline & 43.0 & 58.41 & 61.60 & 87.84 & 91.32 & 92.01 & 92.00 & 91.31 & 87.83 & 61.49 & 57.91 \\
\hline & 44.0 & 58.31 & 61.54 & 86.10 & 89.37 & 90.04 & 90.03 & 89.36 & 86.08 & 61.46 & 58.07 \\
\hline & 45.0 & 58.08 & 59.66 & 61.08 & 62.11 & 62.80 & 62.80 & 62.10 & 61.06 & 59.62 & 57.95 \\
\hline
\end{tabular}


Table 32: Temperature $\left({ }^{\circ} \mathrm{C}\right)$ map of the west side of position B at EOC 145A (106.4 cumulative).

\begin{tabular}{|c|c|c|c|c|c|c|c|c|c|c|c|}
\hline \multirow{2}{*}{$\begin{array}{c}\text { Plate } \\
\text { Location }\end{array}$} & \multirow{2}{*}{$\begin{array}{c}\text { Axial } \\
\text { Location (in) }\end{array}$} & \multicolumn{10}{|c|}{ Width of Plate (inches) } \\
\hline & & 0.00 & 0.08 & 0.16 & 0.25 & 0.50 & 1.00 & 1.50 & 2.00 & 2.08 & 2.16 \\
\hline \multirow{9}{*}{ B-1 } & 0.0 & 51.67 & 51.79 & 51.76 & 51.77 & 51.77 & 51.77 & 51.77 & 51.76 & 51.79 & 51.67 \\
\hline & 1.0 & 53.29 & 53.88 & 75.39 & 77.75 & 77.82 & 77.82 & 77.75 & 75.39 & 53.86 & 53.17 \\
\hline & 2.0 & 53.55 & 54.21 & 78.26 & 80.86 & 80.95 & 80.95 & 80.86 & 78.26 & 54.17 & 53.11 \\
\hline & 3.0 & 53.79 & 54.40 & 78.70 & 81.37 & 81.47 & 81.47 & 81.37 & 78.70 & 54.35 & 53.07 \\
\hline & 4.0 & 54.08 & 54.68 & 80.76 & 83.59 & 83.71 & 83.71 & 83.59 & 80.75 & 54.65 & 53.39 \\
\hline & 5.0 & 54.34 & 54.98 & 82.85 & 85.86 & 85.99 & 85.99 & 85.86 & 82.85 & 54.93 & 53.50 \\
\hline & 6.0 & 54.63 & 55.33 & 85.24 & 88.44 & 88.58 & 88.58 & 88.44 & 85.24 & 55.27 & 53.70 \\
\hline & 7.0 & 54.68 & 53.23 & 55.81 & 56.65 & 56.80 & 56.80 & 56.65 & 55.80 & 53.17 & 53.75 \\
\hline & 7.5 & 54.73 & 52.76 & 53.02 & 53.24 & 53.36 & 53.36 & 53.23 & 53.02 & 52.69 & 53.77 \\
\hline \multirow{9}{*}{ B-2 } & 7.5 & 54.73 & 52.76 & 53.02 & 53.24 & 53.36 & 53.36 & 53.23 & 53.02 & 52.69 & 53.77 \\
\hline & 8.0 & 54.91 & 53.37 & 56.08 & 56.97 & 57.12 & 57.12 & 56.97 & 56.08 & 53.30 & 53.90 \\
\hline & 9.0 & 55.36 & 55.87 & 87.64 & 91.02 & 91.19 & 91.19 & 91.02 & 87.64 & 55.84 & 55.39 \\
\hline & 10.0 & 55.60 & 56.08 & 88.42 & 91.86 & 92.05 & 92.05 & 91.86 & 88.41 & 56.03 & 55.32 \\
\hline & 11.0 & 55.87 & 56.32 & 89.26 & 92.77 & 92.98 & 92.97 & 92.77 & 89.25 & 56.24 & 55.02 \\
\hline & 12.0 & 56.10 & 56.51 & 89.75 & 93.31 & 93.52 & 93.52 & 93.30 & 89.74 & 56.42 & 54.79 \\
\hline & 13.0 & 56.31 & 56.68 & 89.95 & 93.52 & 93.75 & 93.75 & 93.52 & 89.94 & 56.60 & 55.08 \\
\hline & 14.0 & 56.51 & 56.77 & 90.42 & 93.99 & 94.22 & 94.22 & 93.99 & 90.41 & 56.69 & 55.17 \\
\hline & 15.0 & 56.47 & 53.96 & 54.46 & 54.88 & 55.13 & 55.13 & 54.87 & 54.45 & 53.86 & 55.18 \\
\hline \multirow{9}{*}{ B-3 } & 15.0 & 56.47 & 53.96 & 54.46 & 54.88 & 55.13 & 55.13 & 54.87 & 54.45 & 53.86 & 55.18 \\
\hline & 16.0 & 56.95 & 57.17 & 91.20 & 94.86 & 95.10 & 95.09 & 94.85 & 91.19 & 57.06 & 55.54 \\
\hline & 17.0 & 57.14 & 57.39 & 91.80 & 95.50 & 95.76 & 95.76 & 95.50 & 91.79 & 57.32 & 56.77 \\
\hline & 18.0 & 57.33 & 57.57 & 91.99 & 95.72 & 95.99 & 95.99 & 95.71 & 91.98 & 57.52 & 57.30 \\
\hline & 19.0 & 57.53 & 57.77 & 92.20 & 95.95 & 96.24 & 96.24 & 95.94 & 92.19 & 57.69 & 57.10 \\
\hline & 20.0 & 57.69 & 57.92 & 92.24 & 95.99 & 96.30 & 96.30 & 95.99 & 92.23 & 57.81 & 56.60 \\
\hline & 21.0 & 57.86 & 58.09 & 92.25 & 96.00 & 96.32 & 96.32 & 96.00 & 92.23 & 57.98 & 56.51 \\
\hline & 22.0 & 57.81 & 55.77 & 59.09 & 60.36 & 60.73 & 60.73 & 60.35 & 59.07 & 55.66 & 56.29 \\
\hline & 22.5 & 57.80 & 55.21 & 55.88 & 56.44 & 56.79 & 56.78 & 56.43 & 55.86 & 55.16 & 57.92 \\
\hline \multirow{9}{*}{ B-4 } & 22.5 & 57.80 & 55.21 & 55.88 & 56.44 & 56.79 & 56.78 & 56.43 & 55.86 & 55.16 & 57.92 \\
\hline & 23.0 & 57.93 & 55.88 & 59.20 & 60.46 & 60.83 & 60.83 & 60.45 & 59.18 & 55.78 & 56.41 \\
\hline & 24.0 & 58.27 & 58.55 & 93.02 & 96.82 & 97.15 & 97.15 & 96.82 & 93.01 & 58.42 & 56.63 \\
\hline & 25.0 & 58.38 & 58.71 & 93.01 & 96.82 & 97.18 & 97.17 & 96.81 & 92.99 & 58.58 & 57.24 \\
\hline & 26.0 & 58.49 & 58.91 & 93.30 & 97.15 & 97.54 & 97.54 & 97.14 & 93.29 & 58.82 & 58.18 \\
\hline & 27.0 & 58.60 & 59.06 & 93.28 & 97.14 & 97.54 & 97.54 & 97.13 & 93.26 & 58.99 & 58.55 \\
\hline & 28.0 & 58.68 & 59.22 & 93.42 & 97.31 & 97.72 & 97.72 & 97.30 & 93.40 & 59.13 & 58.26 \\
\hline & 29.0 & 58.71 & 59.29 & 93.56 & 97.43 & 97.84 & 97.84 & 97.42 & 93.54 & 59.16 & 57.58 \\
\hline & 30.0 & 58.54 & 56.48 & 57.35 & 58.04 & 58.48 & 58.47 & 58.03 & 57.32 & 56.35 & 57.09 \\
\hline \multirow{9}{*}{ B-5 } & 30.0 & 58.54 & 56.48 & 57.35 & 58.04 & 58.48 & 58.48 & 58.03 & 57.32 & 56.35 & 57.09 \\
\hline & 31.0 & 58.82 & 59.47 & 92.11 & 95.82 & 96.21 & 96.20 & 95.81 & 92.08 & 59.35 & 57.57 \\
\hline & 32.0 & 58.87 & 59.62 & 92.01 & 95.72 & 96.14 & 96.13 & 95.71 & 91.99 & 59.50 & 57.43 \\
\hline & 33.0 & 58.90 & 59.77 & 92.13 & 95.85 & 96.28 & 96.28 & 95.83 & 92.10 & 59.63 & 57.35 \\
\hline & 34.0 & 58.91 & 59.91 & 91.96 & 95.67 & 96.12 & 96.12 & 95.66 & 91.93 & 59.78 & 57.81 \\
\hline & 35.0 & 58.92 & 60.04 & 91.83 & 95.54 & 95.99 & 95.99 & 95.52 & 91.81 & 59.94 & 58.51 \\
\hline & 36.0 & 58.90 & 60.14 & 91.29 & 94.94 & 95.38 & 95.38 & 94.92 & 91.26 & 60.05 & 58.64 \\
\hline & \begin{tabular}{|l|l}
37.0 \\
\end{tabular} & 58.71 & 58.07 & 61.42 & 62.80 & 63.30 & 63.30 & 62.78 & 61.38 & 57.97 & 58.49 \\
\hline & 37.5 & 58.62 & 57.59 & 58.60 & 59.36 & 59.85 & 59.85 & 59.35 & 58.56 & 57.46 & 57.37 \\
\hline \multirow{9}{*}{ B-6 } & 37.5 & 58.62 & 57.59 & 58.60 & 59.36 & 59.85 & 59.85 & 59.35 & 58.56 & 57.46 & 57.37 \\
\hline & 38.0 & 58.66 & 58.13 & 61.36 & 62.70 & 63.20 & 63.19 & 62.68 & 61.32 & 58.00 & 57.69 \\
\hline & 39.0 & 58.77 & 60.39 & 90.82 & 94.40 & 94.83 & 94.82 & 94.39 & 90.79 & 60.26 & 57.62 \\
\hline & 40.0 & 58.72 & 60.39 & 89.39 & 92.85 & 93.27 & 93.27 & 92.83 & 89.35 & 60.27 & 57.50 \\
\hline & 41.0 & 58.65 & 60.38 & 87.75 & 91.07 & 91.51 & 91.50 & 91.06 & 87.72 & 60.26 & 57.60 \\
\hline & 42.0 & 58.56 & 60.37 & 86.20 & 89.40 & 89.84 & 89.83 & 89.38 & 86.17 & 60.24 & 57.39 \\
\hline & 43.0 & 58.48 & 60.42 & 86.07 & 89.22 & 89.66 & 89.66 & 89.21 & 86.03 & 60.30 & 57.67 \\
\hline & 44.0 & 58.34 & 60.32 & 84.26 & 87.24 & 87.67 & 87.66 & 87.22 & 84.22 & 60.23 & 58.03 \\
\hline & 45.0 & 58.11 & 58.42 & 59.53 & 60.32 & 60.82 & 60.82 & 60.29 & 59.49 & 58.35 & 57.99 \\
\hline
\end{tabular}




\section{REFERENCES}

1. D.M. Wachs, "RERTR Fuel Development and Qualification Plan," INL/EXT-05-01017, Revision 4, August 2009.

2. "Experiment Control Plan for the AFIP-4 Fuel Irradiation in the ATR," PLN-3028, February 2009.

3. J. W. Nielsen, G. S. Chang. "As-Run Neutronic Analysis for the AFIP-4 Experiment Irradiated in the Center Flux Trap of the ATR, Cycles 144B and 145A,” ECAR-1466. June 2011.

4. RERTR Project Personnel, "RERTR AFIP-4 Irradiation Experiment in the Advanced Test Reactor: As-Built Data Package," AFIP-4, April 2009.

5. D.M. Wachs, "Hydraulic Testing of the AFIP Irradiation Vehicle for the ATR Center Flux Trap Position," ECAR-99. October 2007.

6. R. G. Ambrosek, "RELAP Analysis for AFIP-2 Flow Resistor,” ECAR-126, January 2008.

7. P. E. Murray, "Validation of ABAQUS Standard 6.7-3 Heat Transfer", ECAR-131, January 2008.

8. R. H. Perry, D. W. Green, "Perry's Chemical Engineer's Handbook," $7^{\text {th }}$ Edition, McGraw-Hill, 1997. 


\section{Appendix A: Individual Plate Power and Fission Density Plots}


Plate L1B31Z
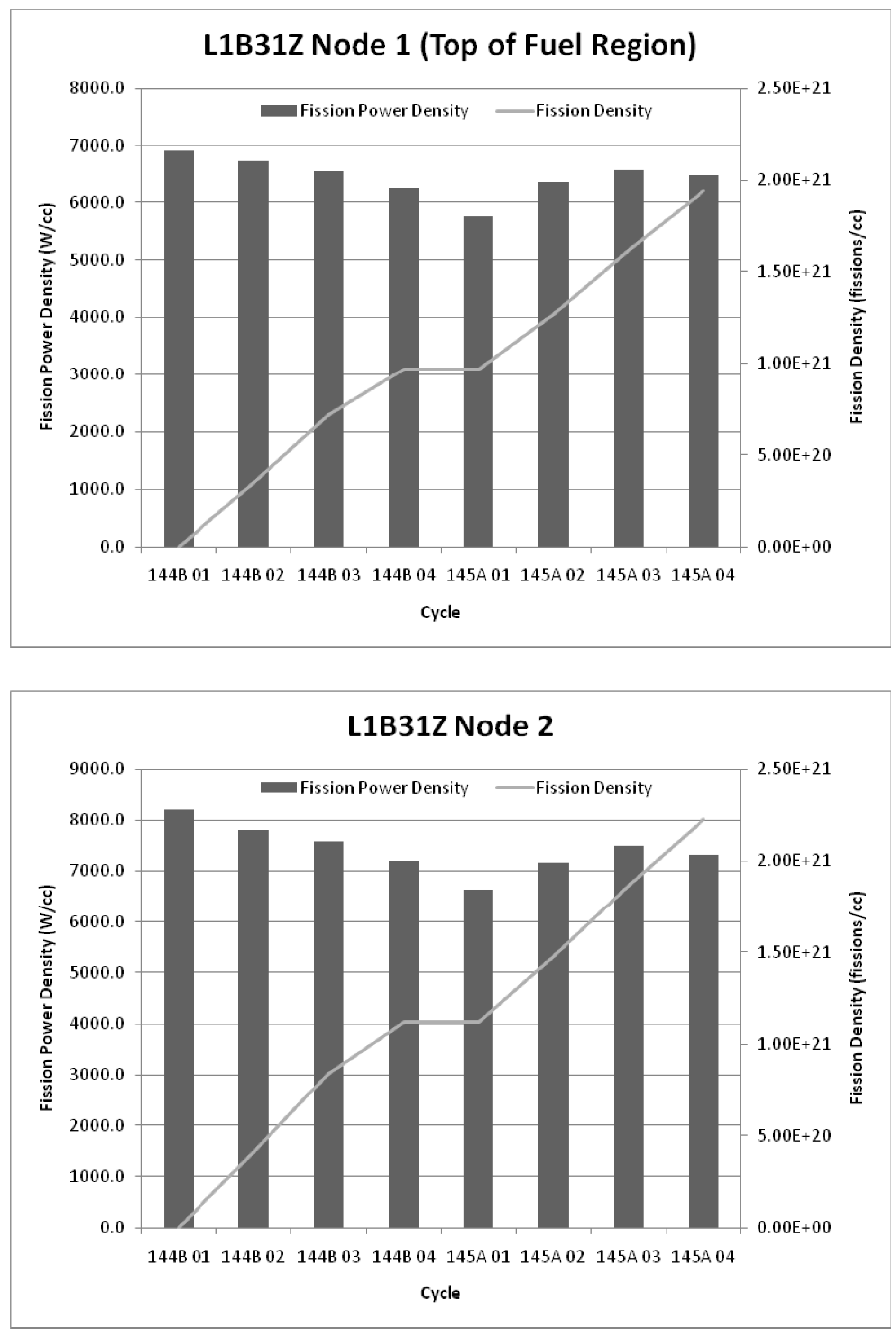

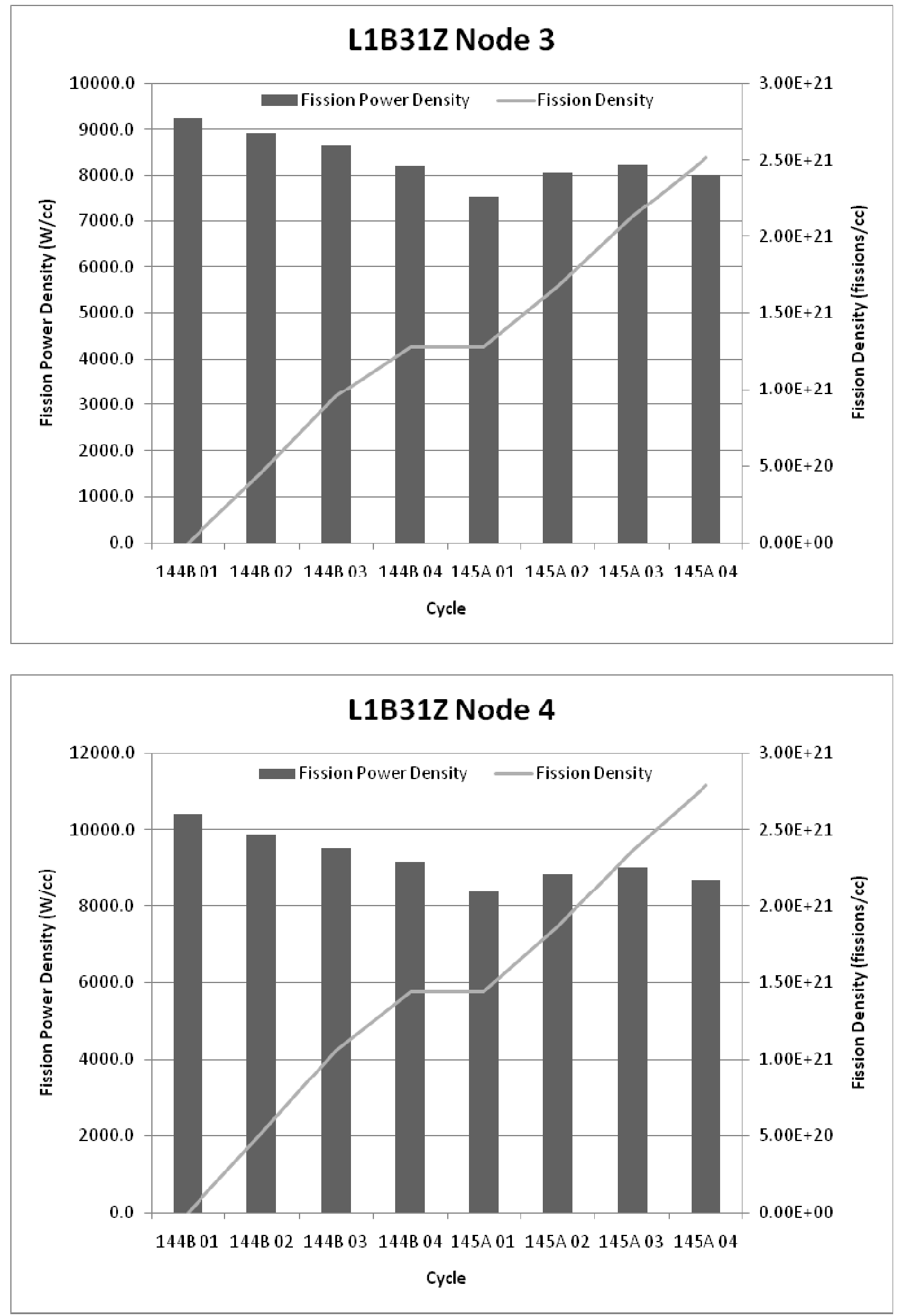


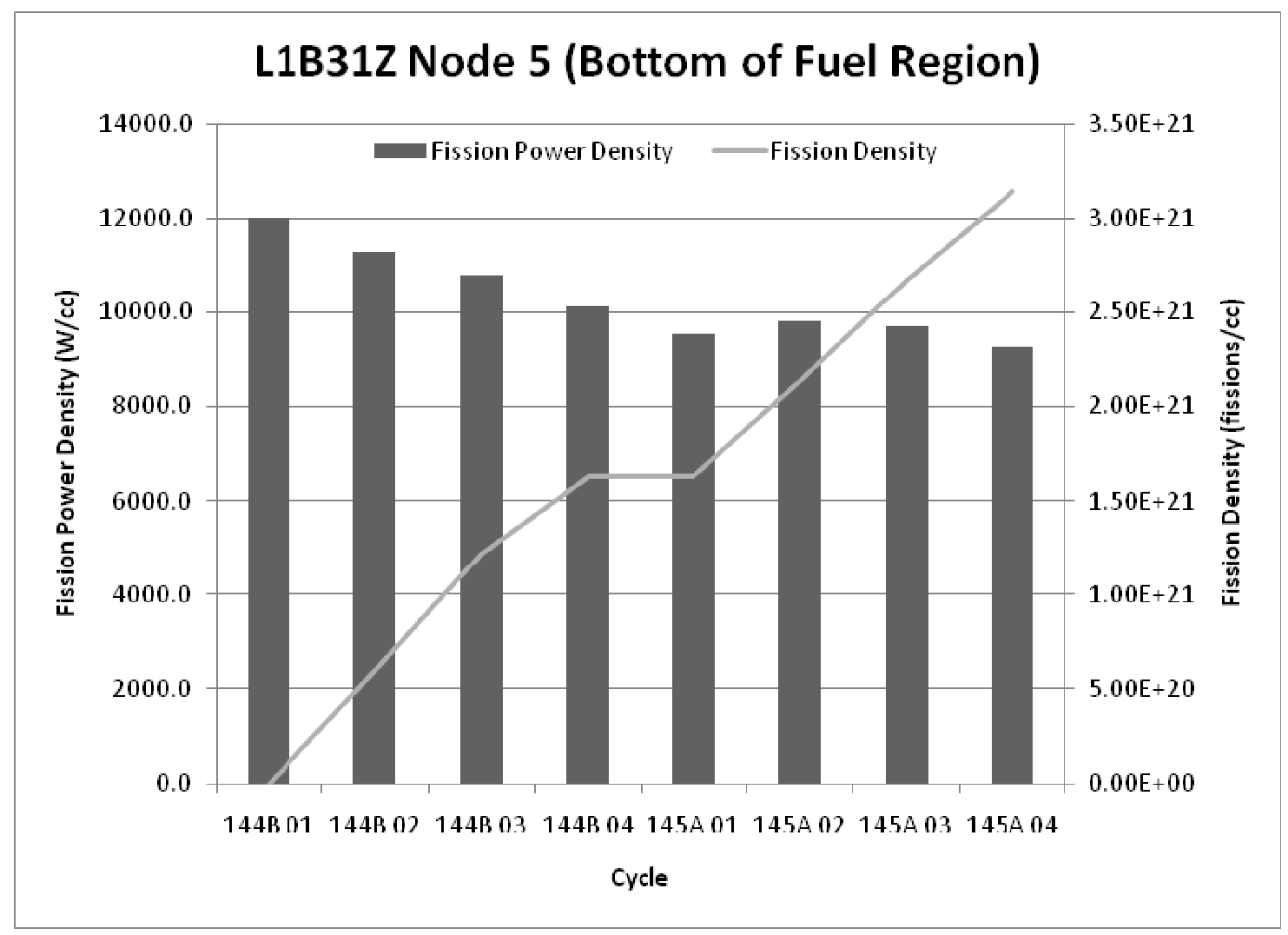




\section{A-1. Plate L1B33Z}
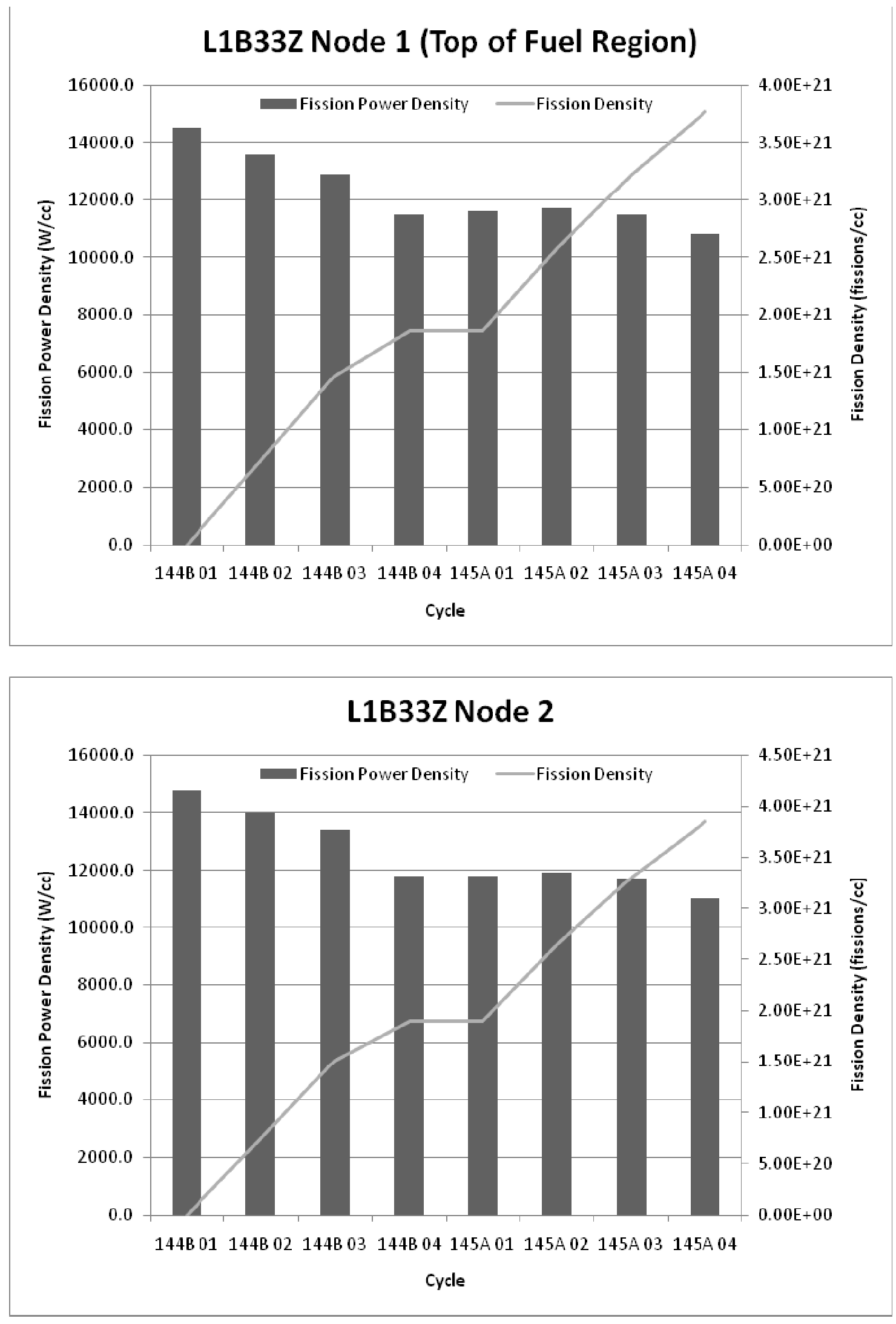

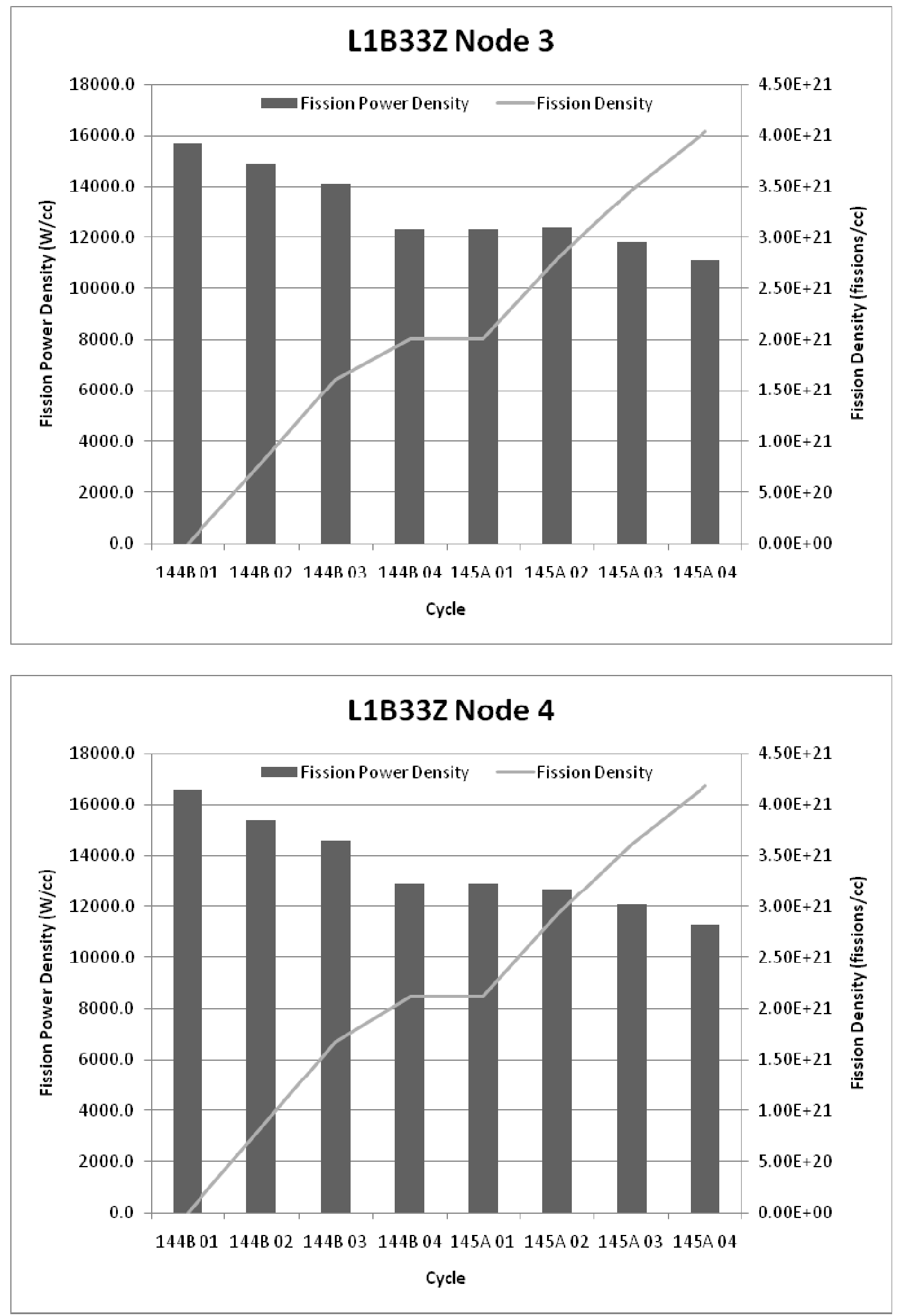


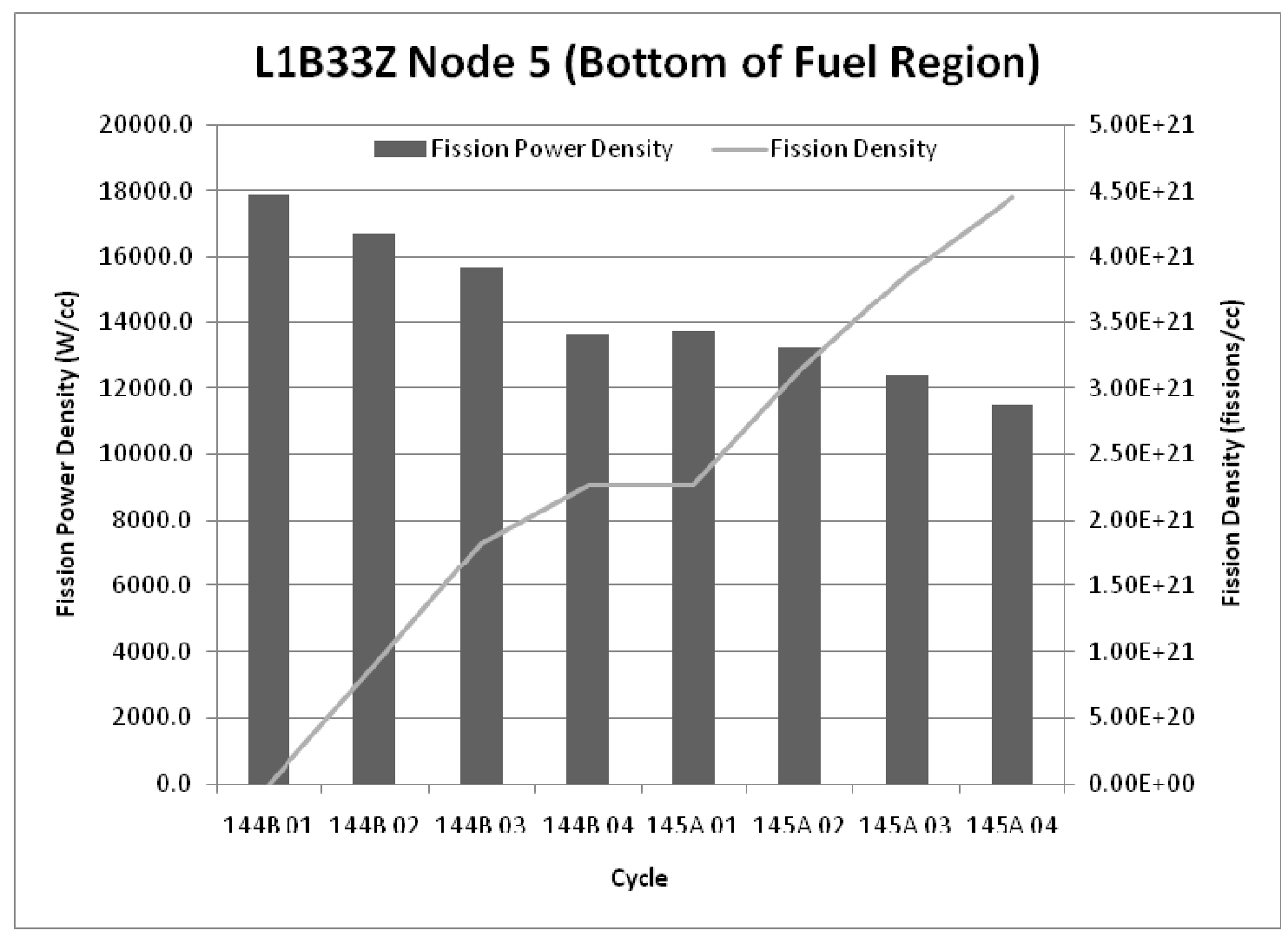




\section{A-2. Plate L1B32Z}
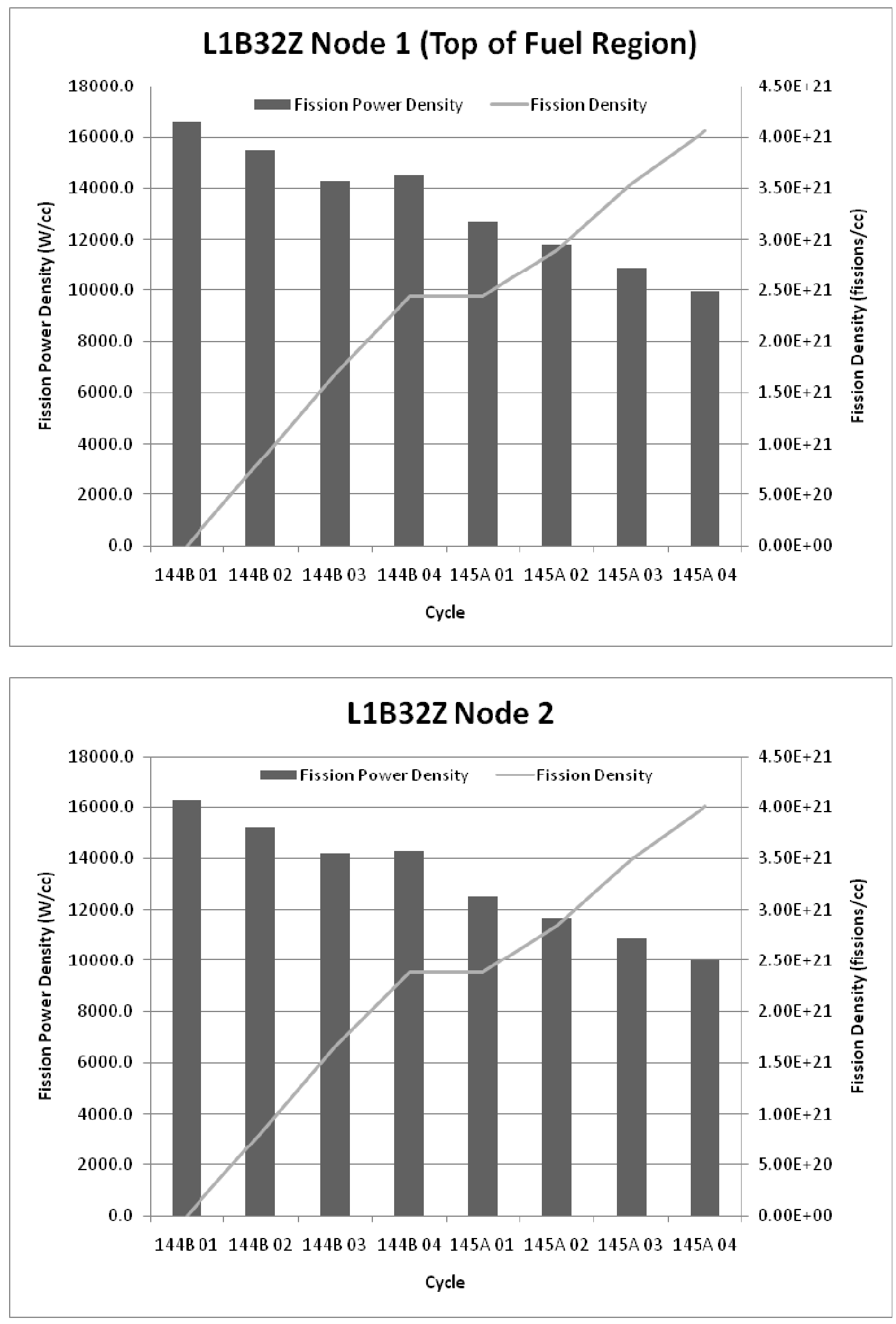

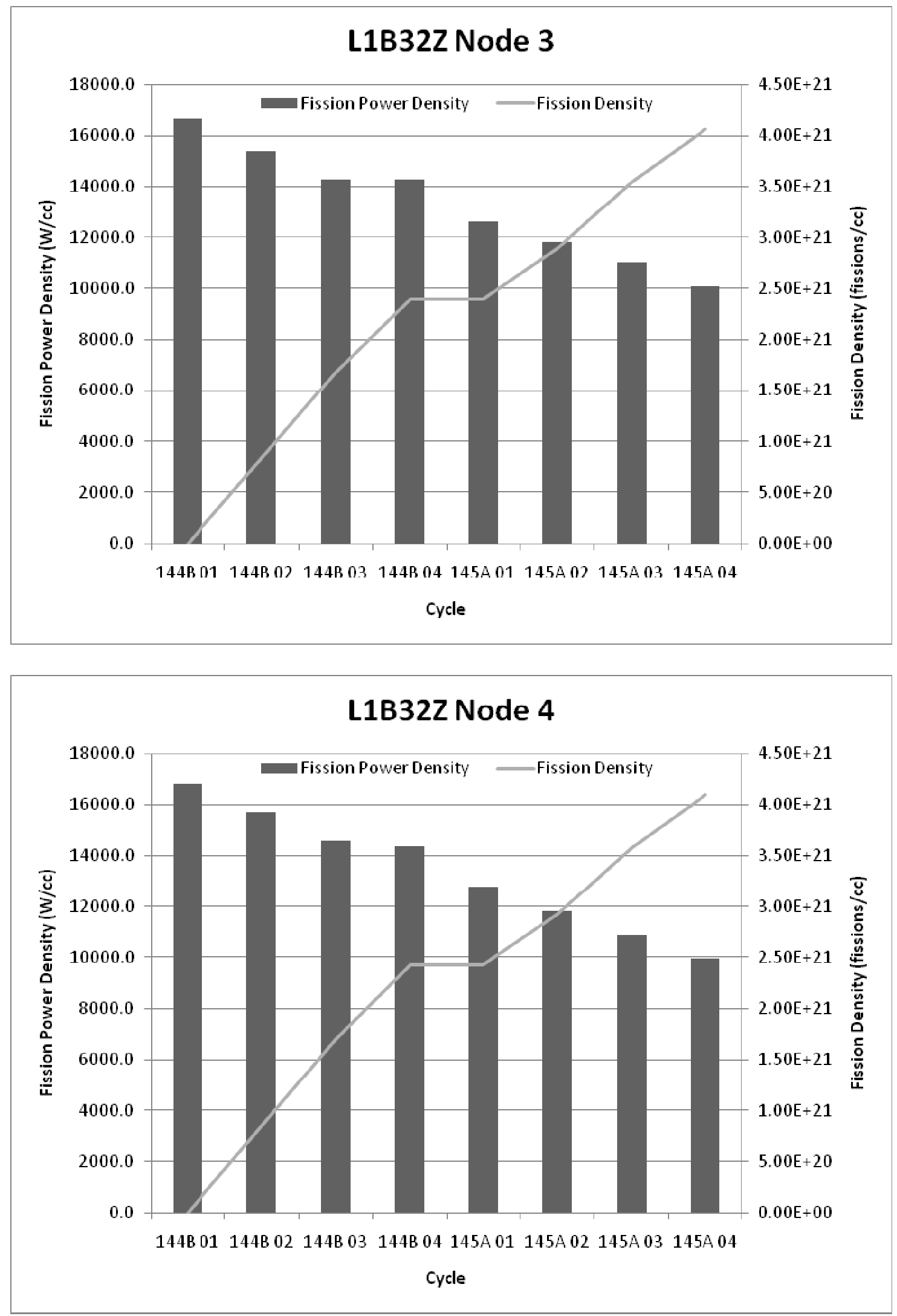


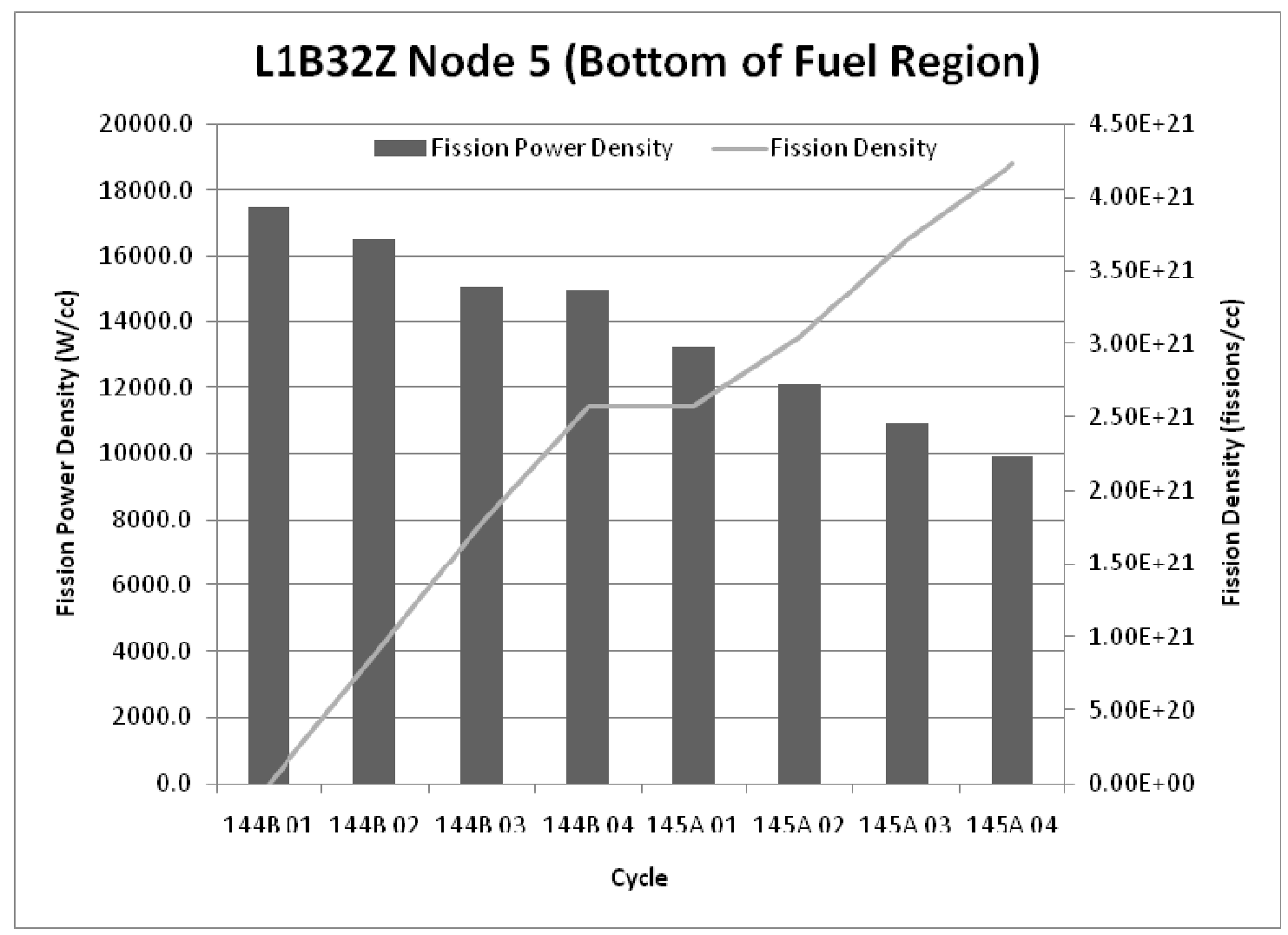




\section{A-3. Plate L1B51Z}
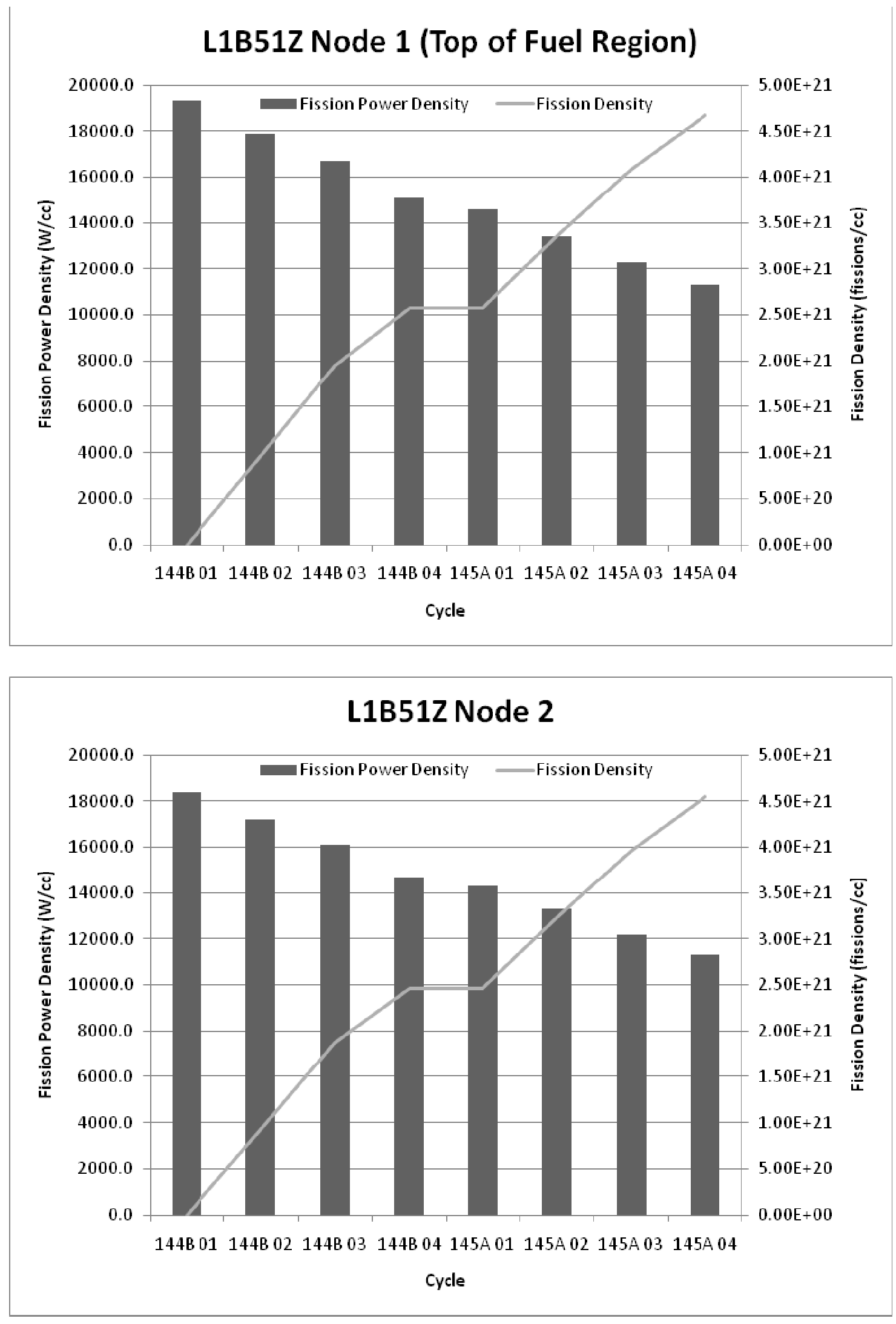

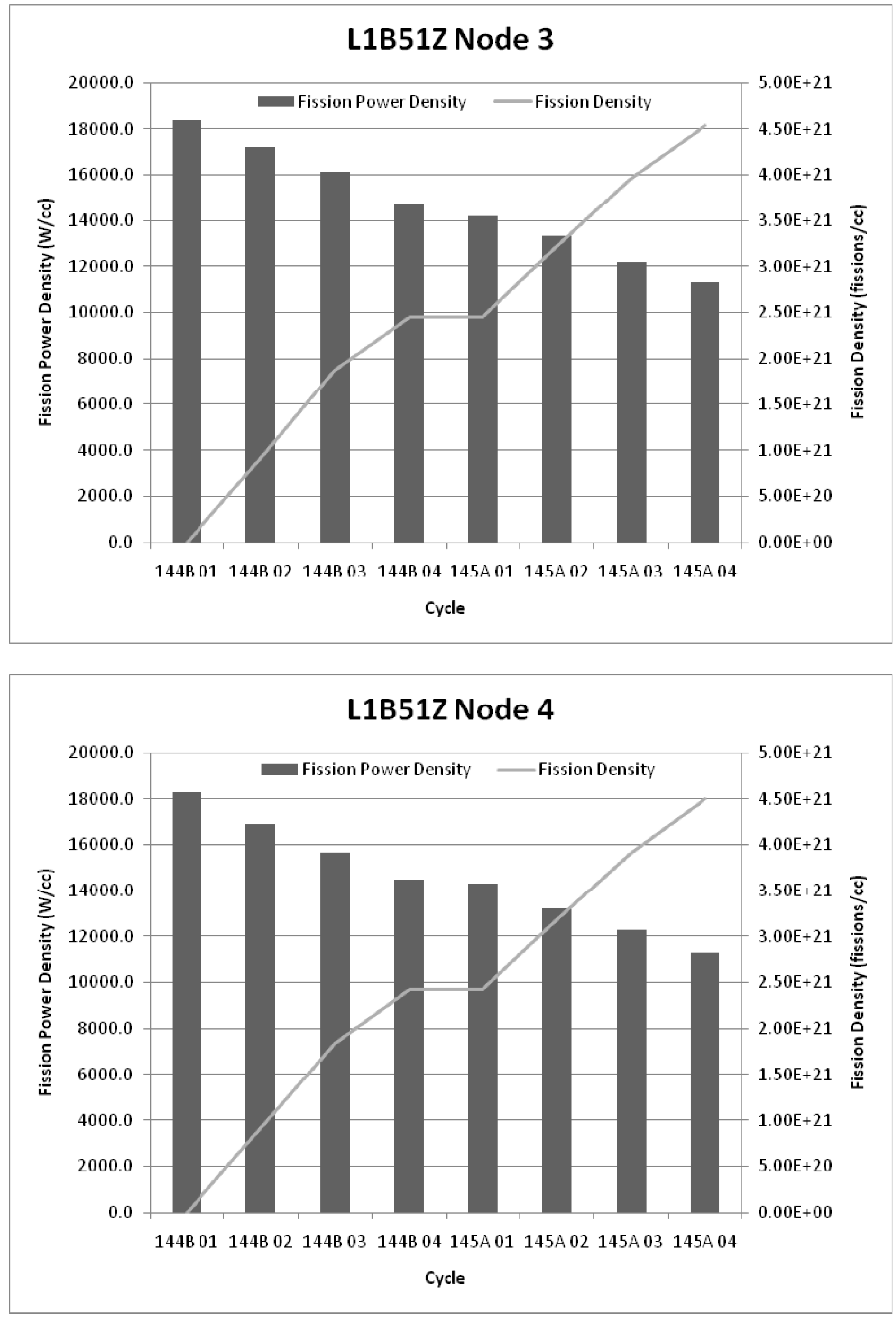


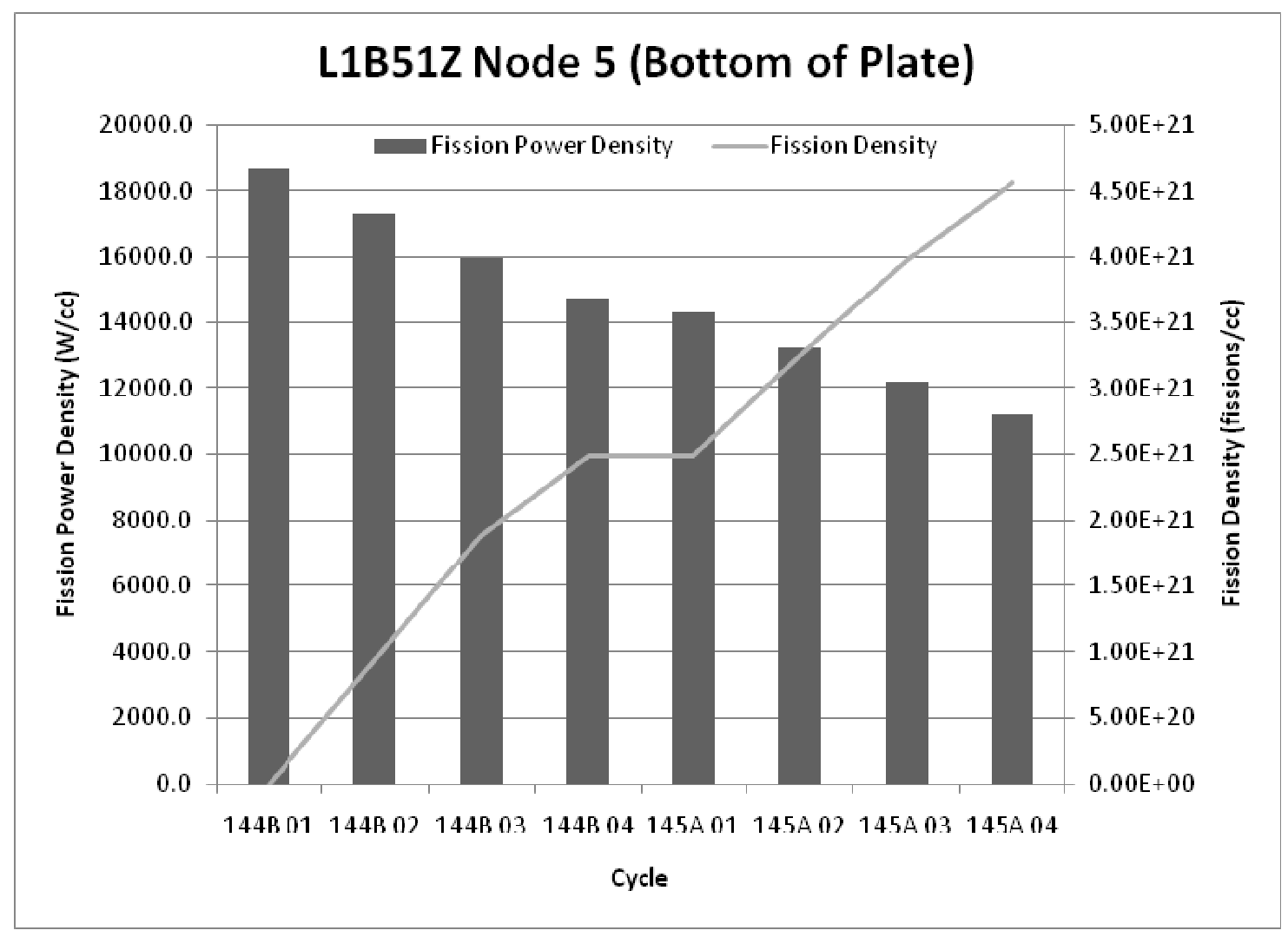




\section{A-4. Plate L1B52Z}
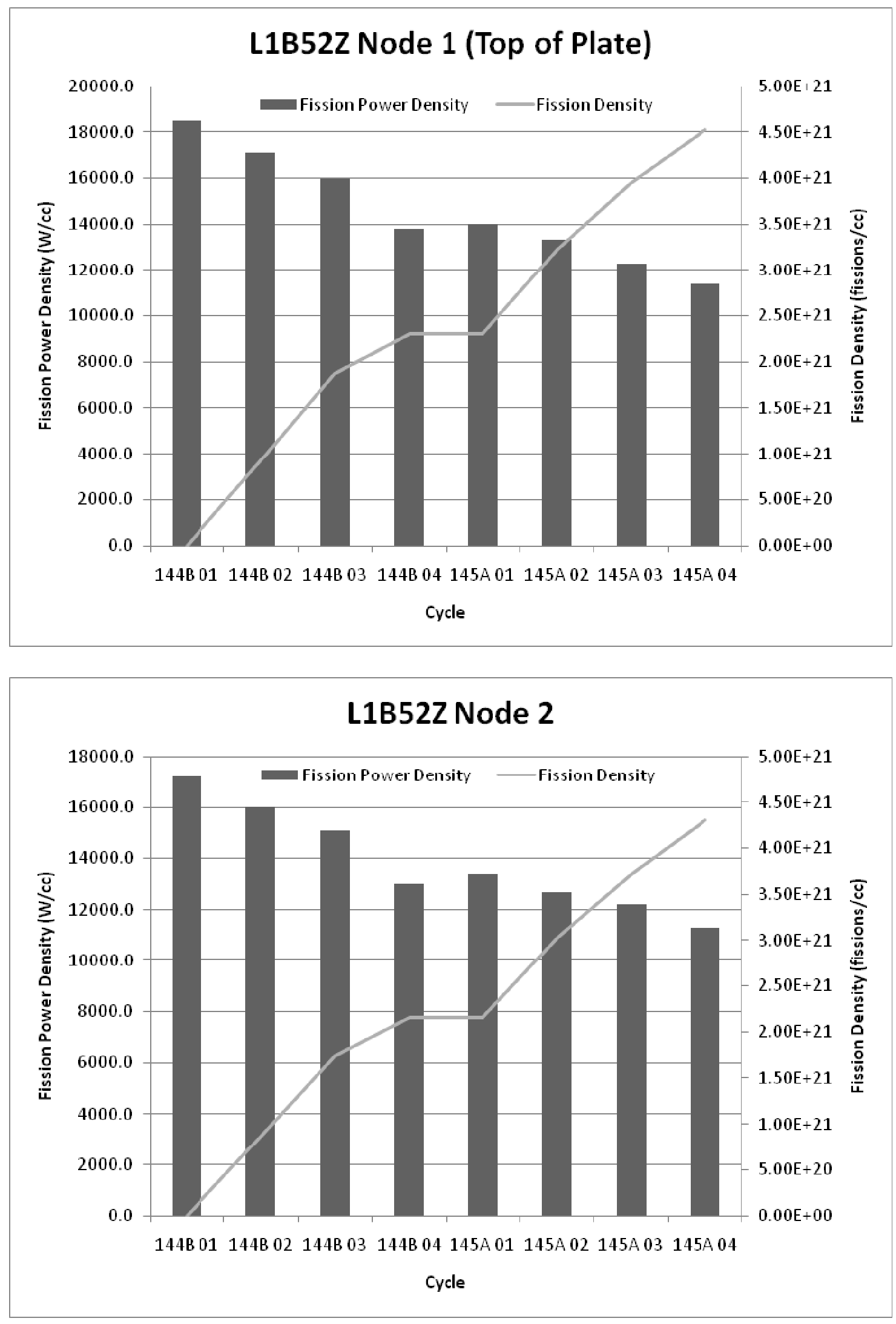

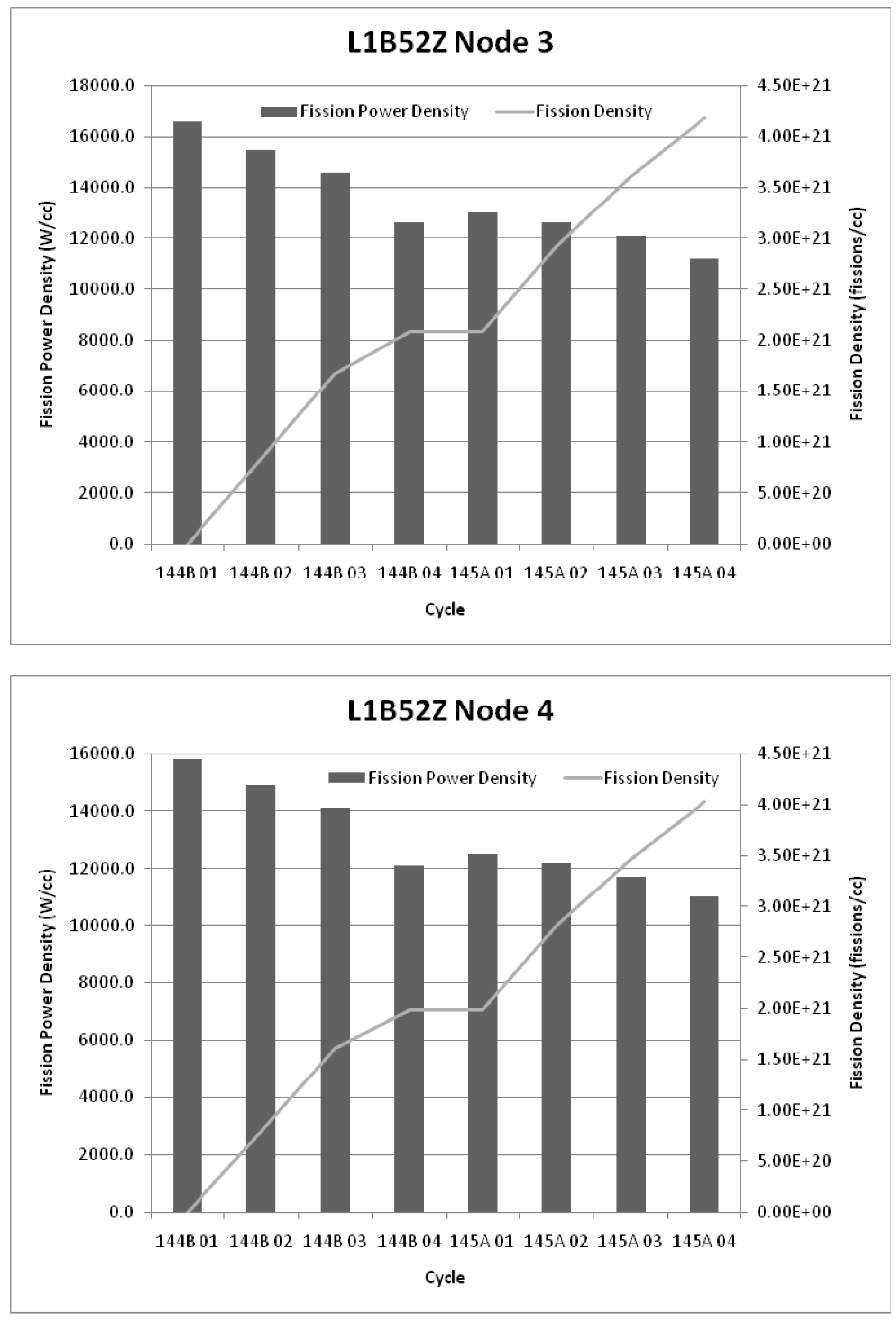


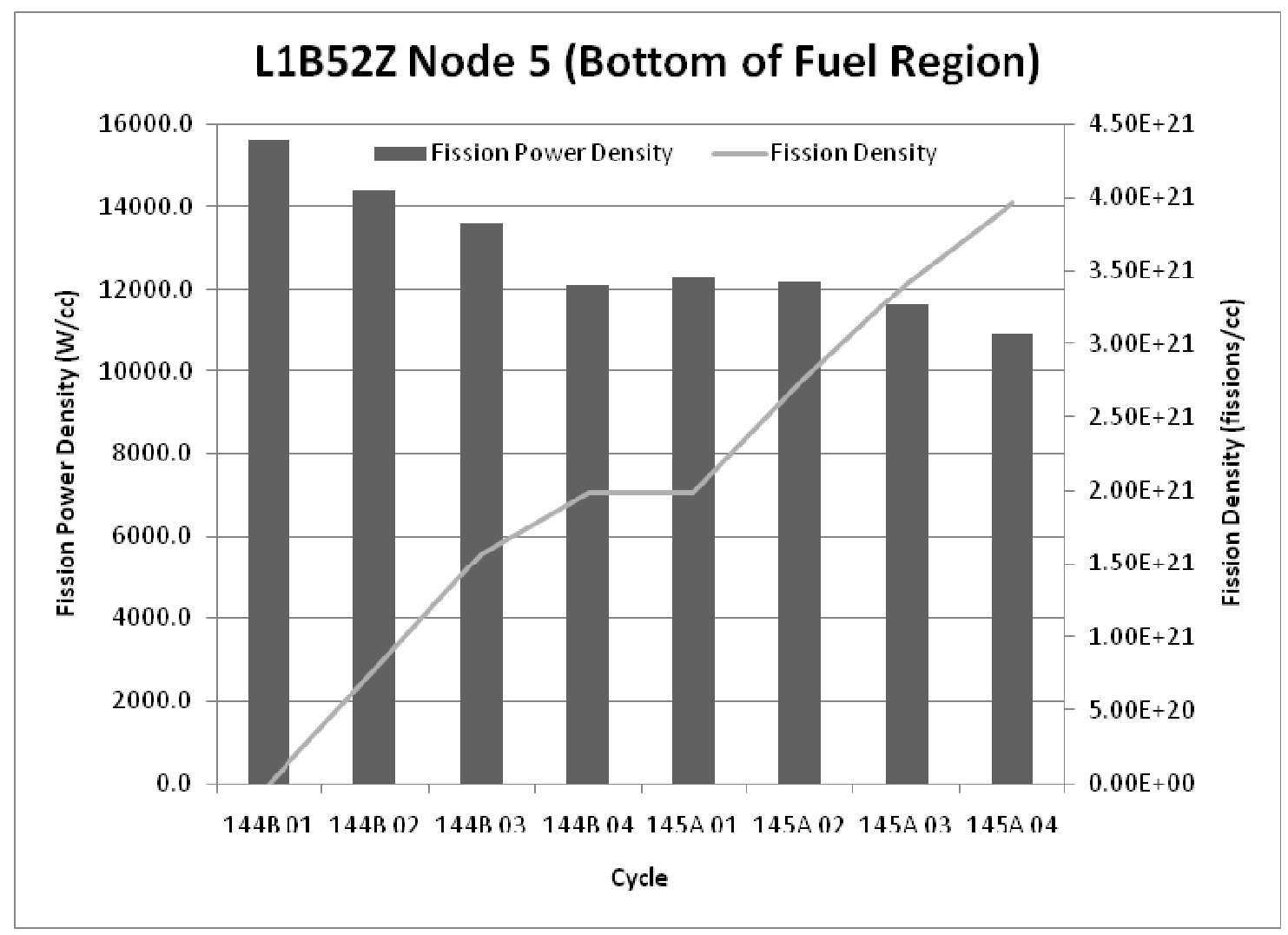




\section{A-5. Plate L1B53Z}
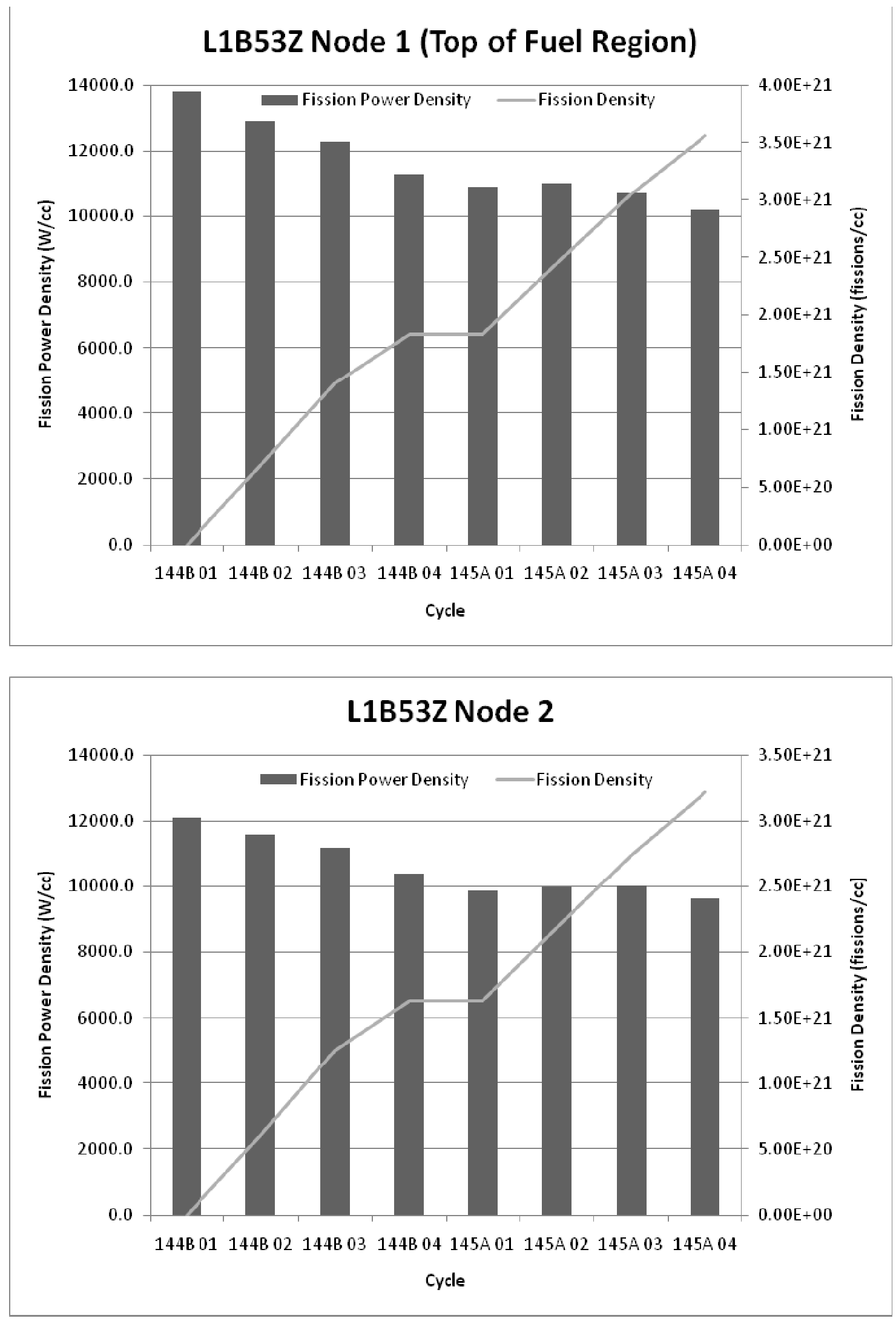

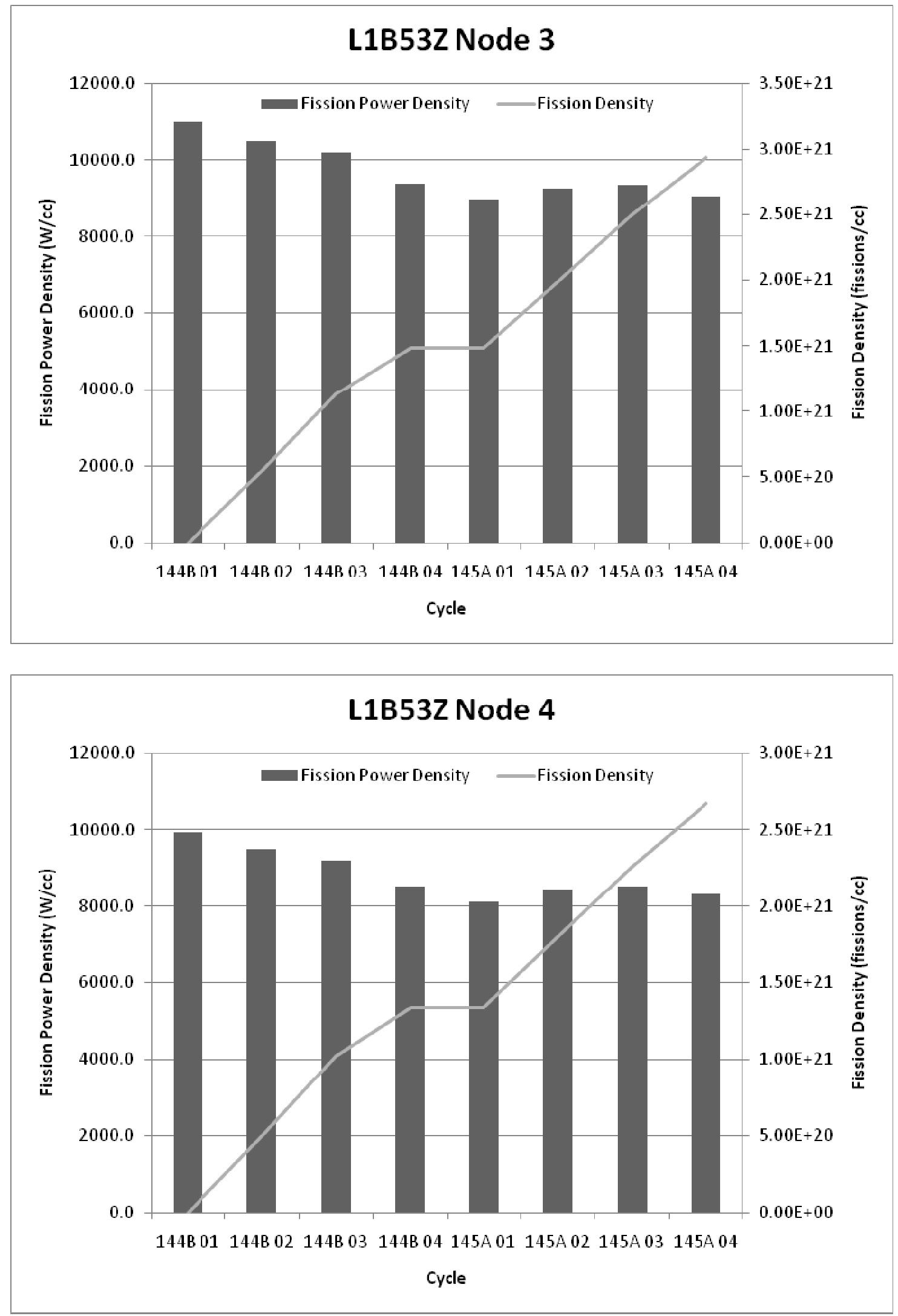


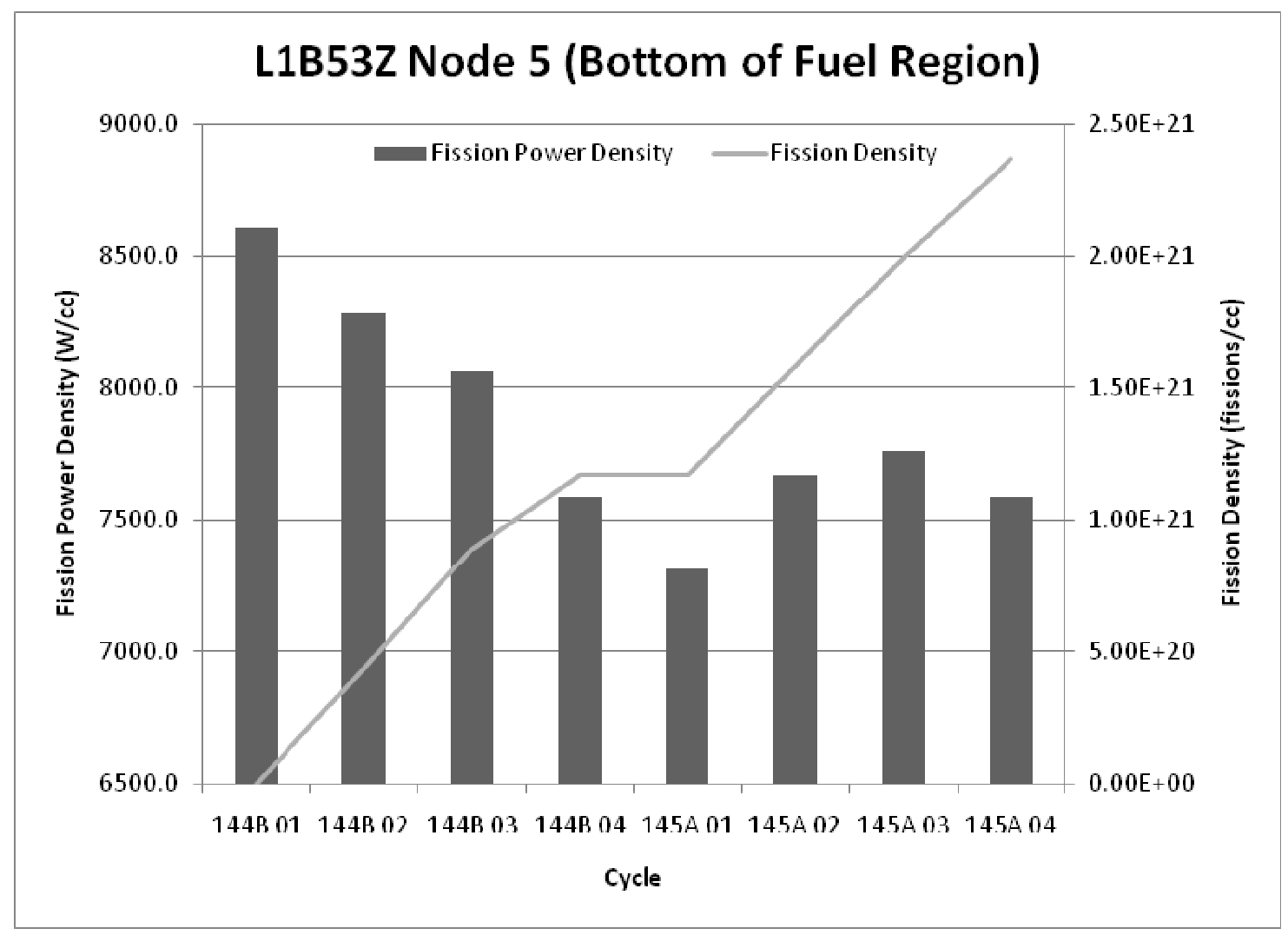




\section{A-6. Plate L1H34Z}
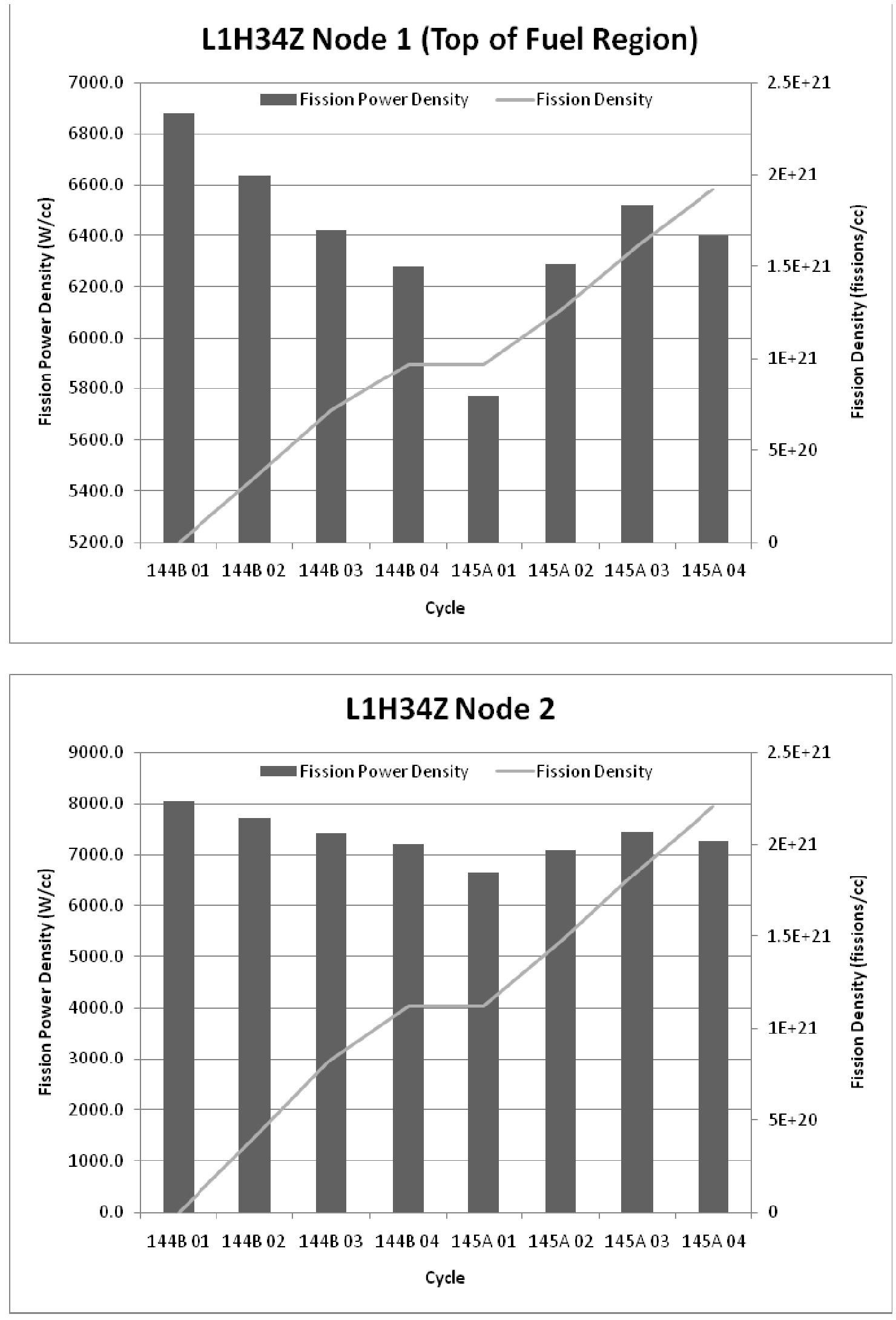

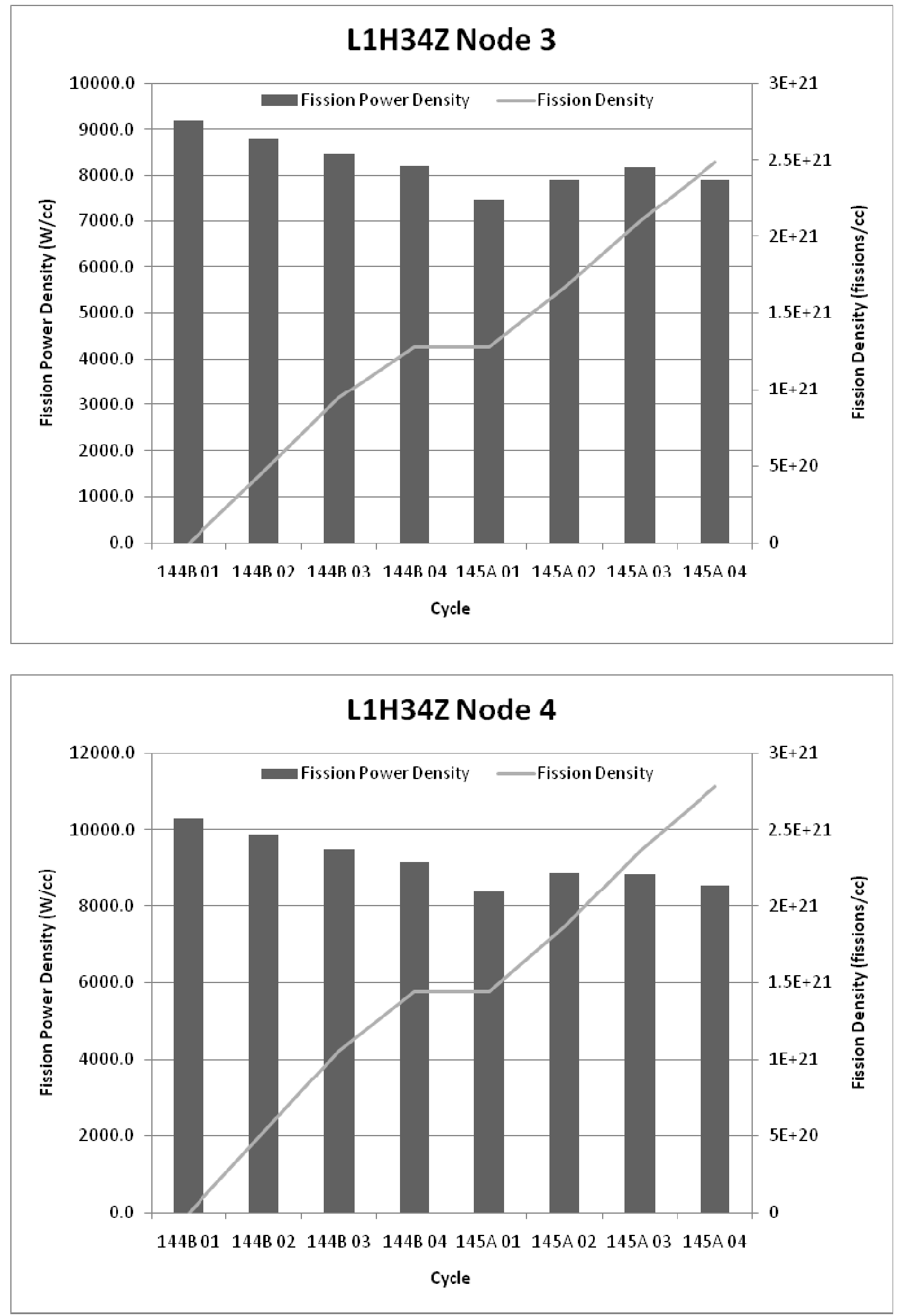


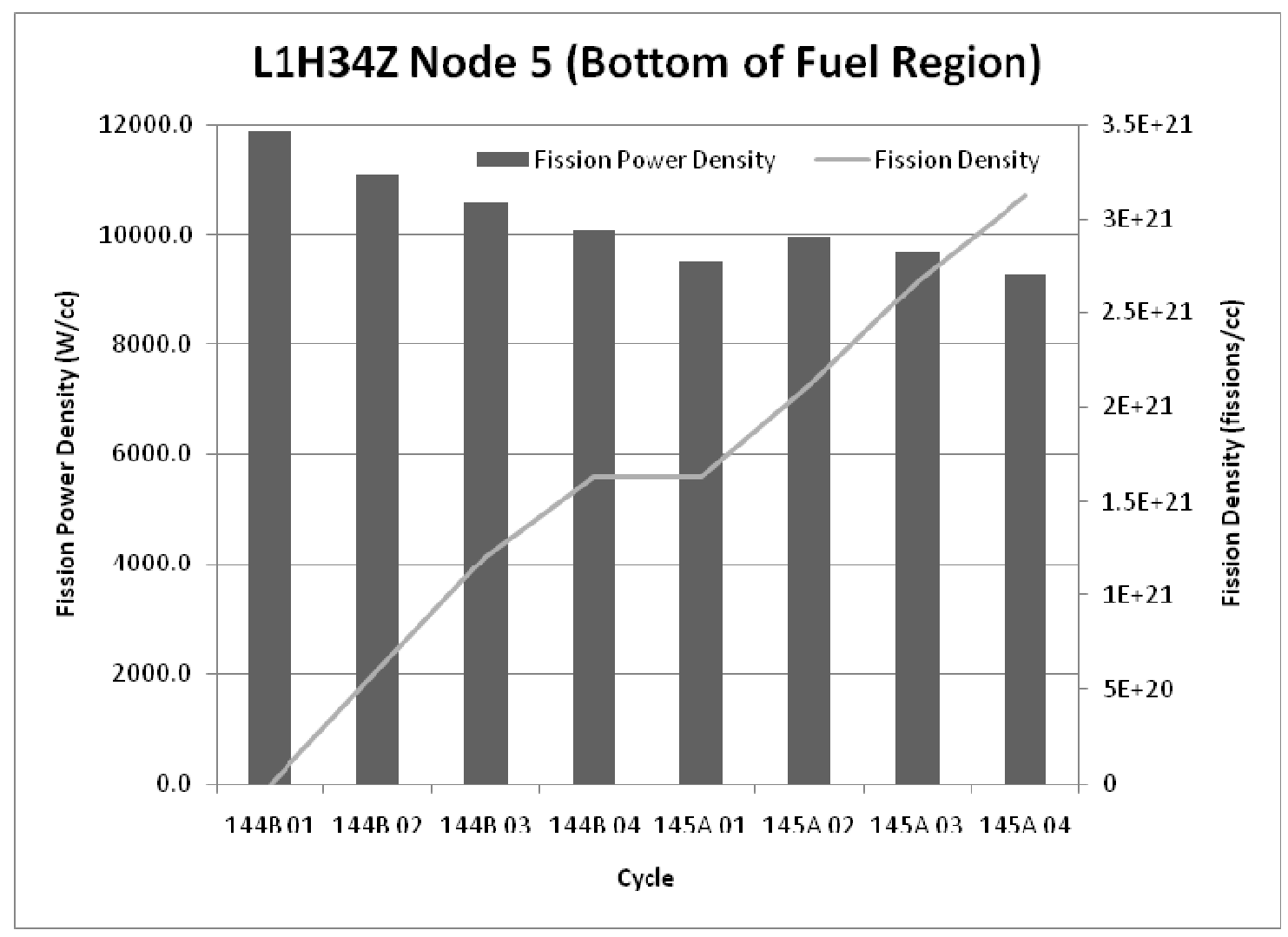




\section{A-7. Plate L1H35Z}
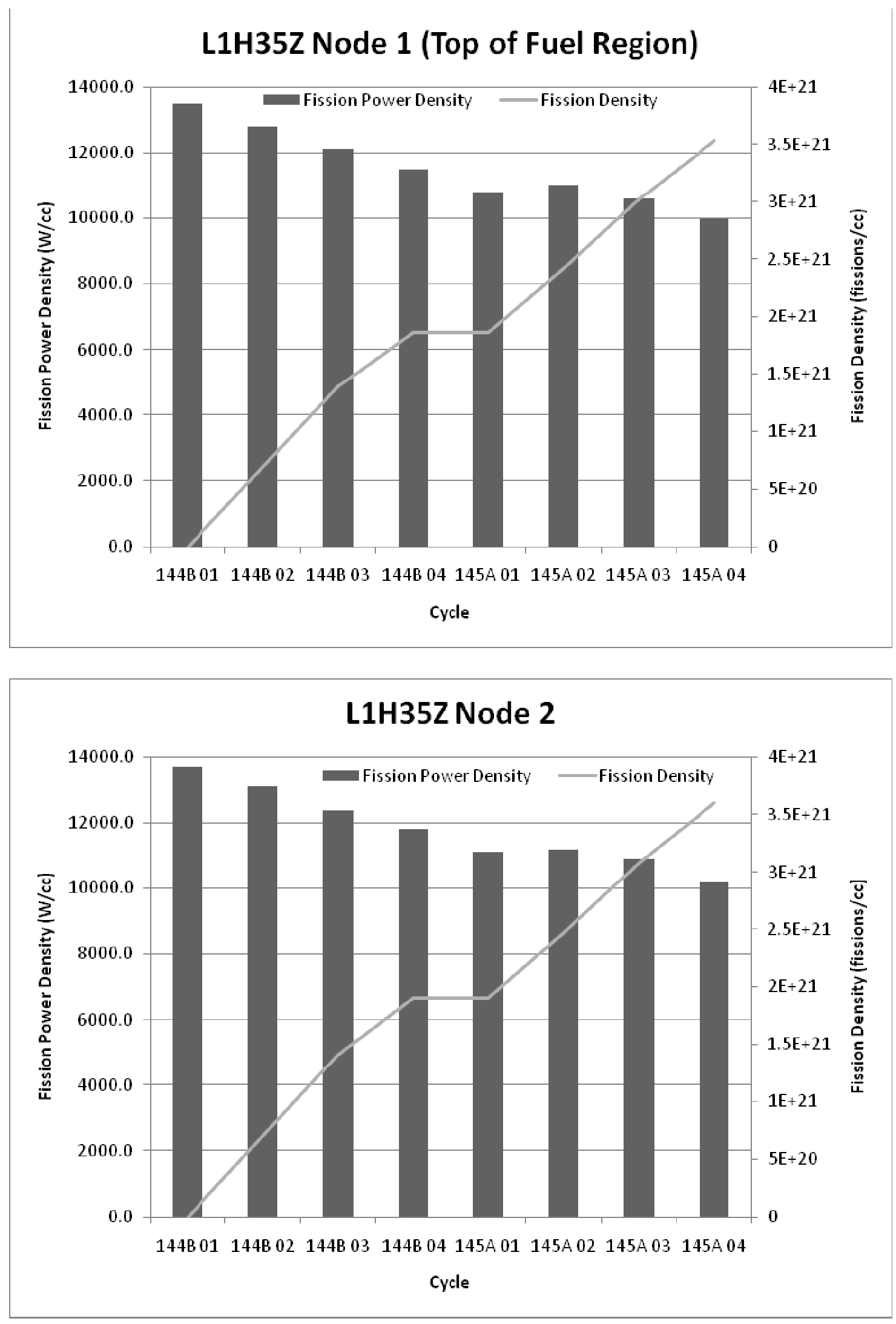

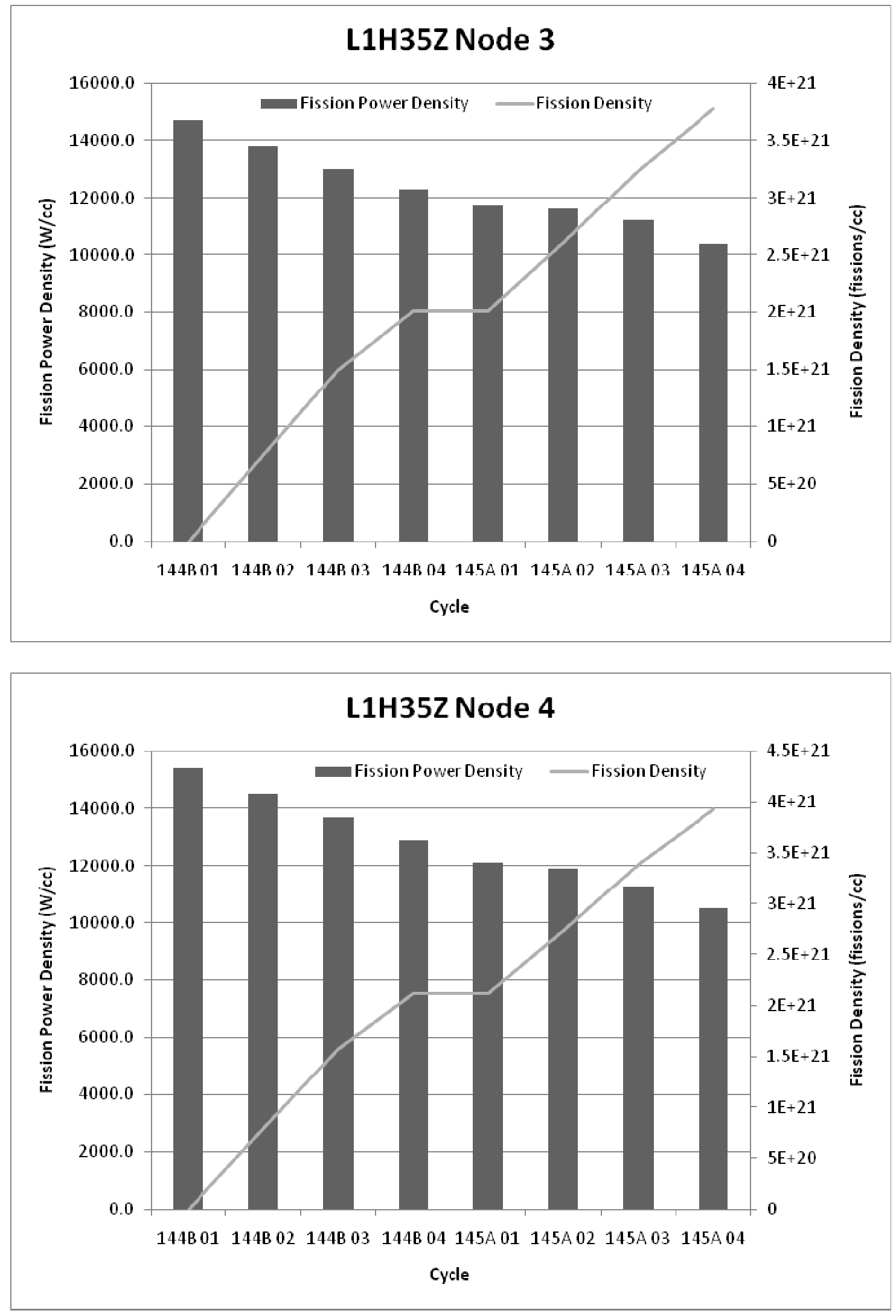


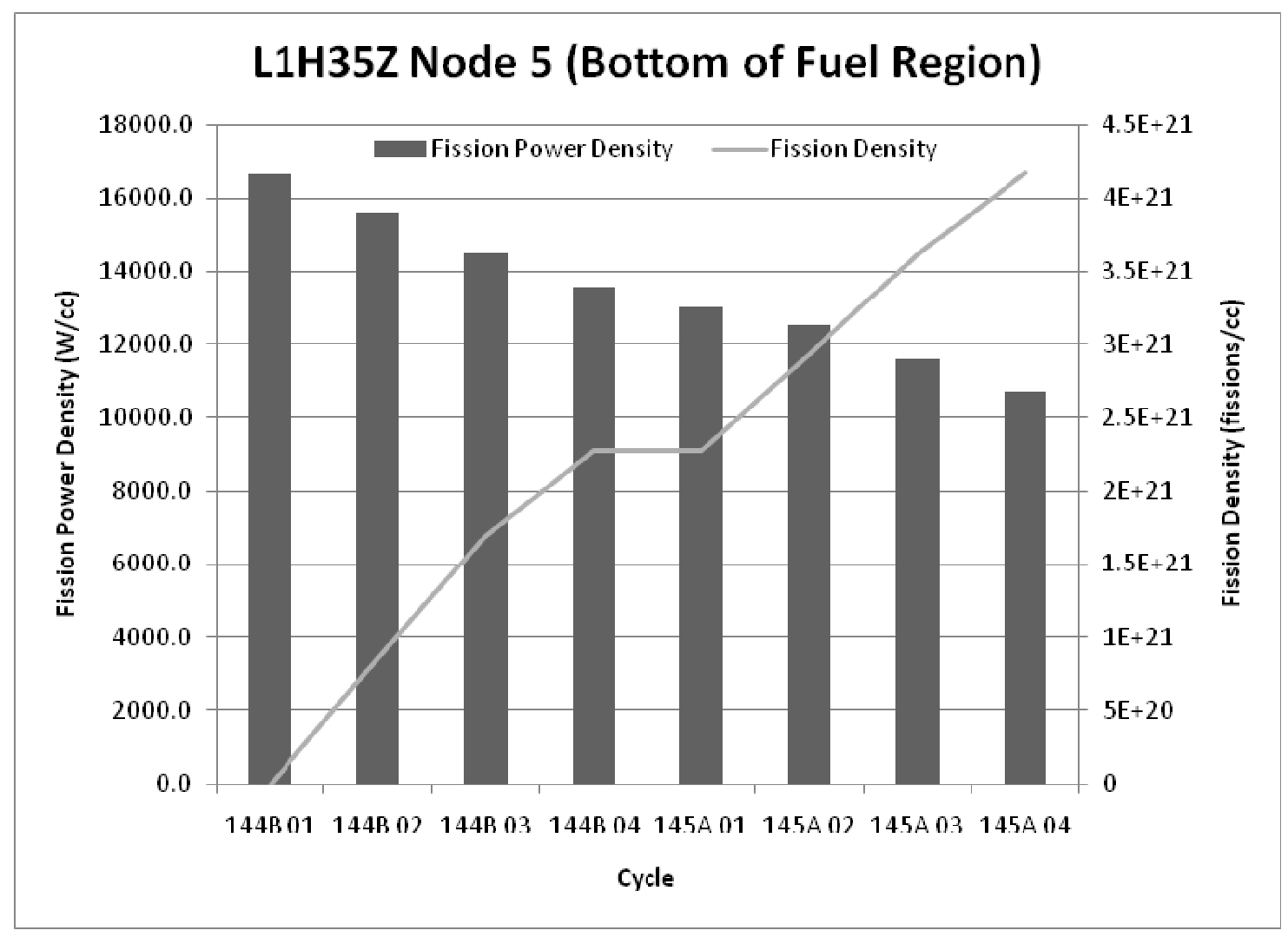




\section{A-8. Plate L1H36Z}
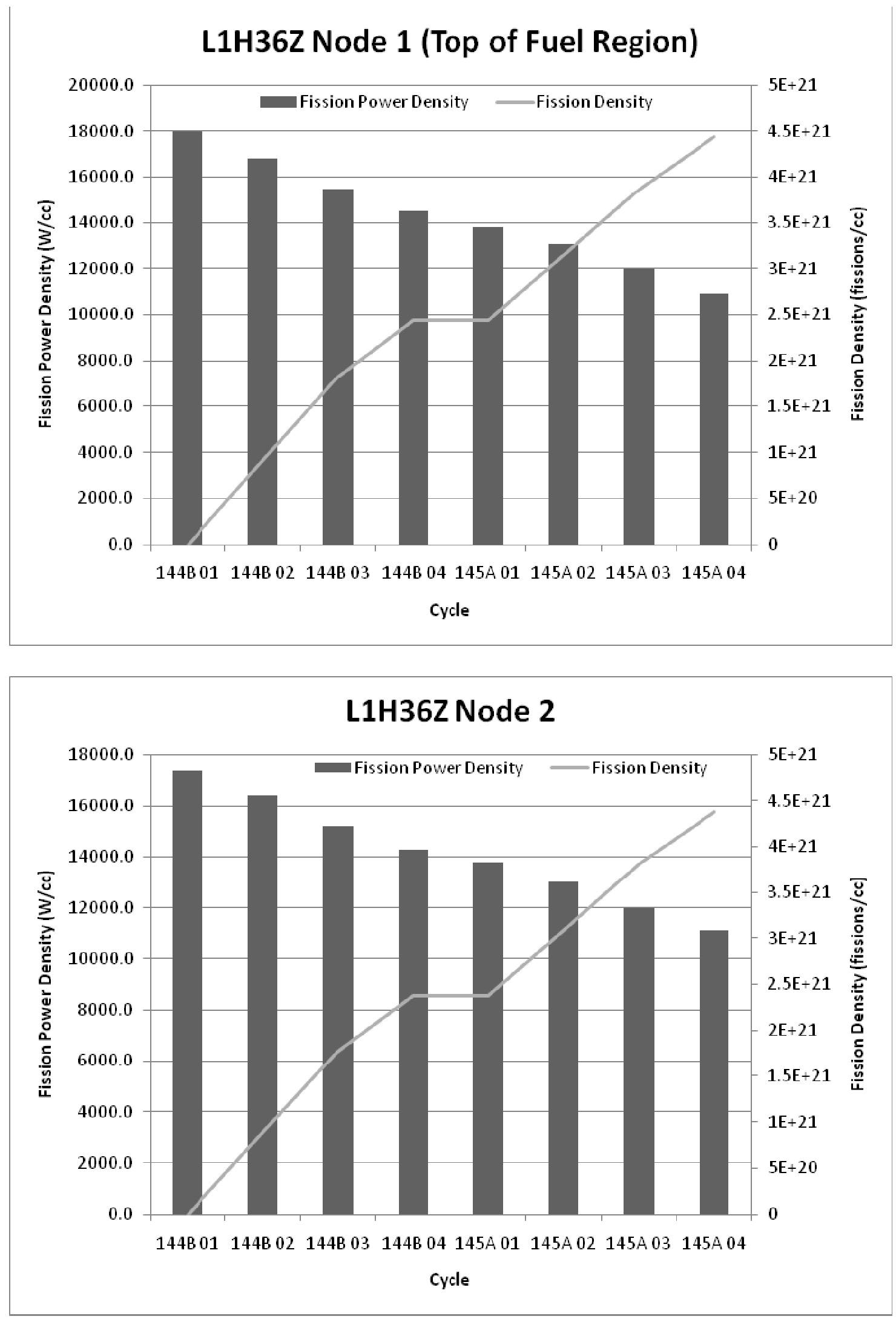

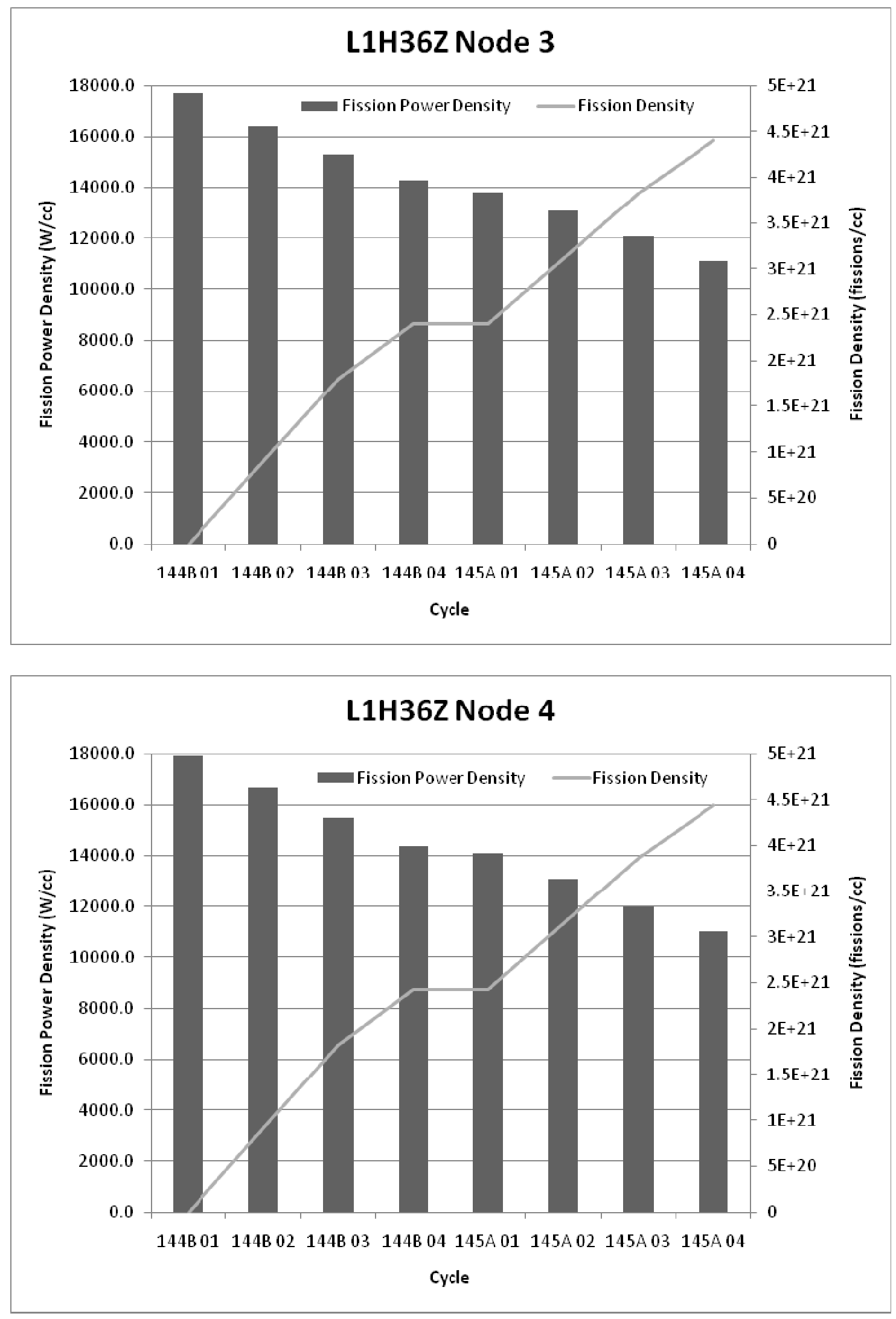


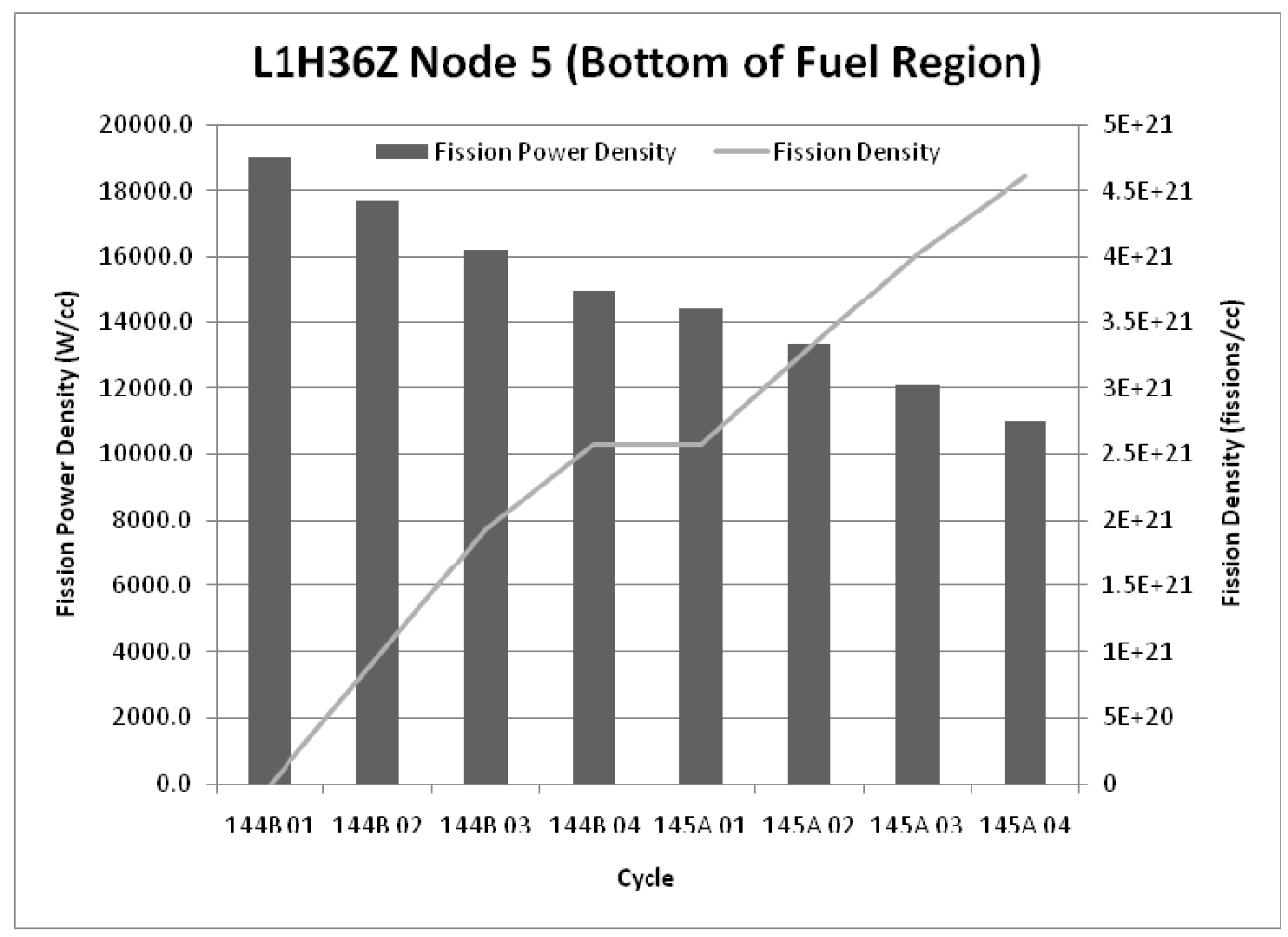




\section{A-9. Plate L1H37Z}
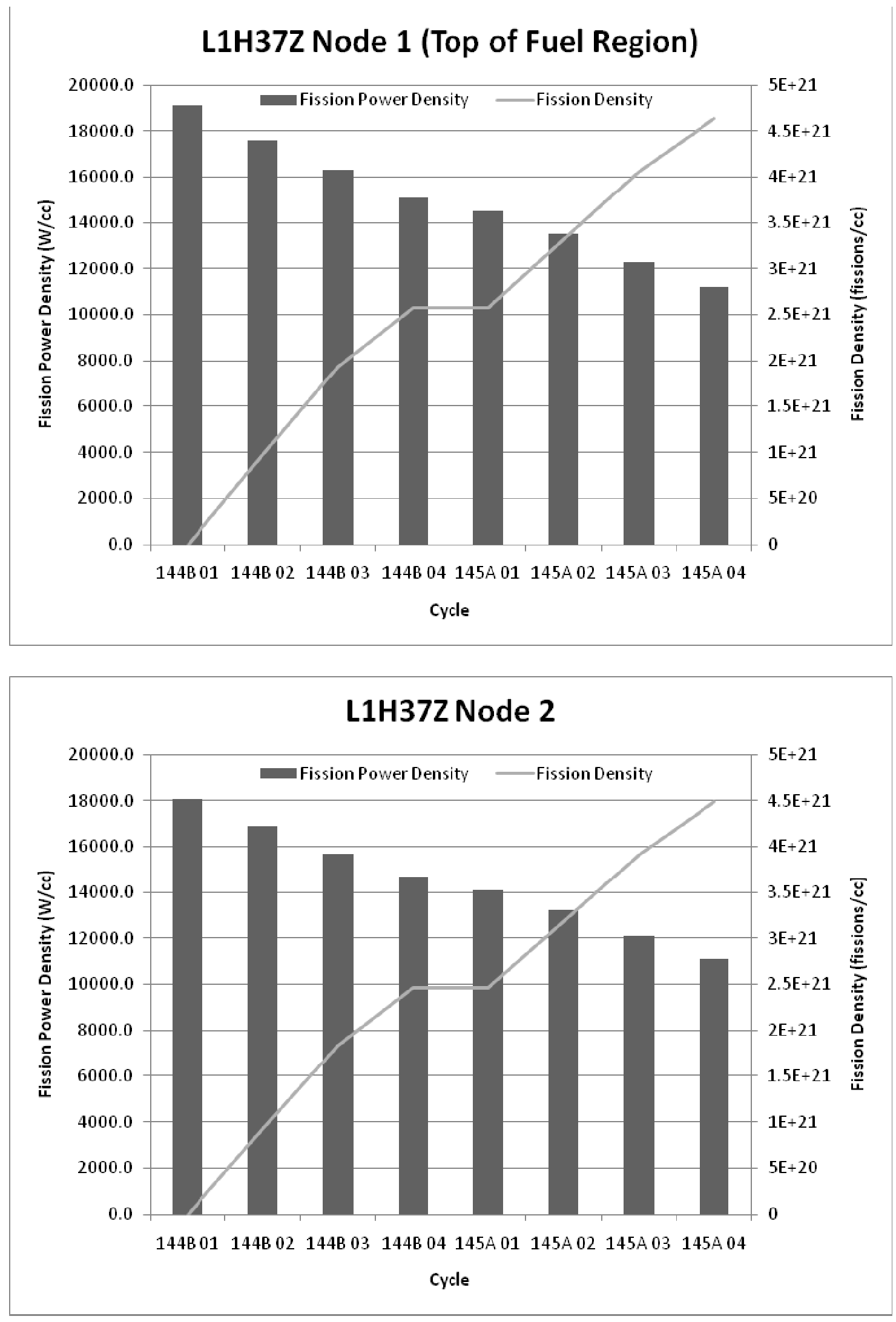

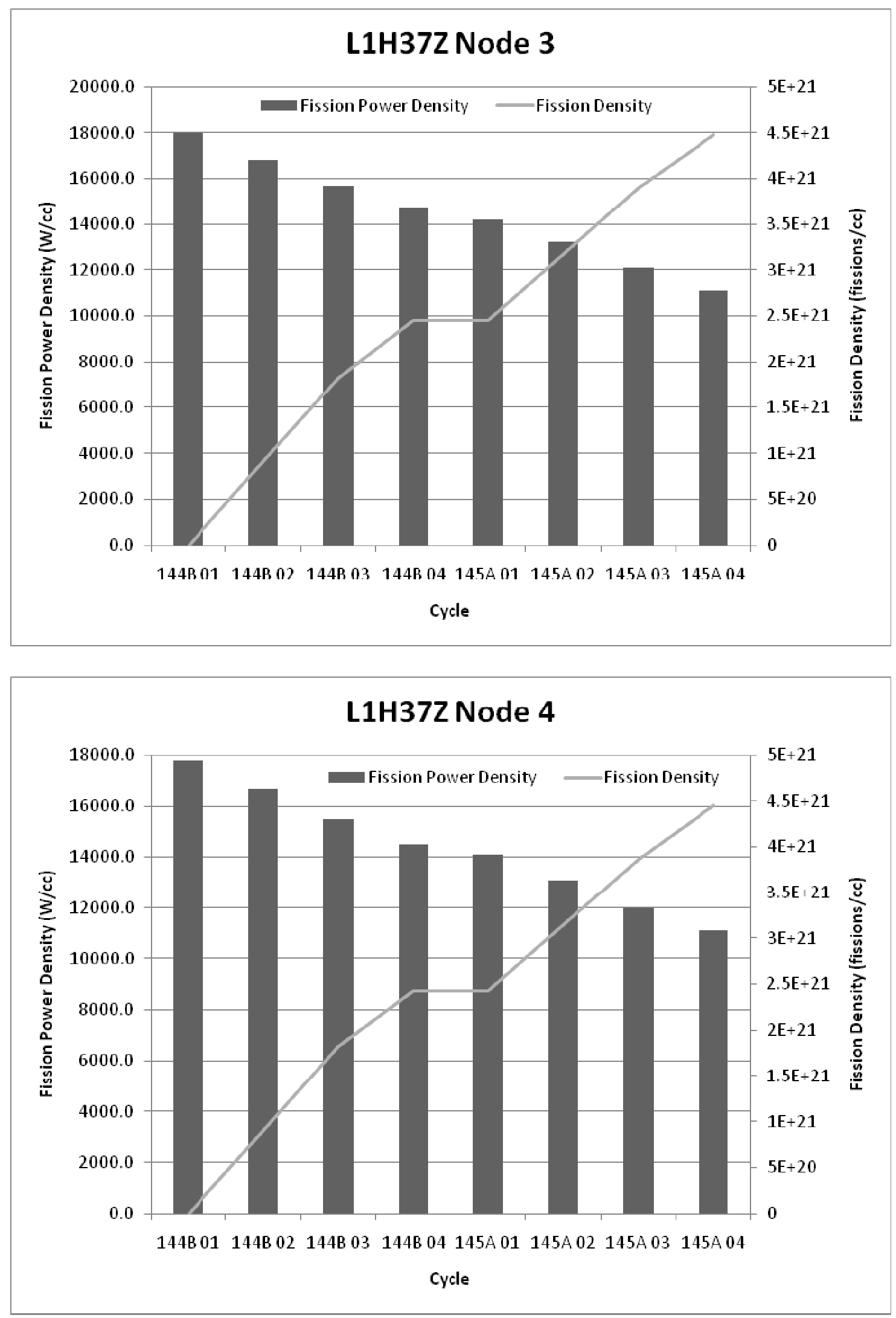


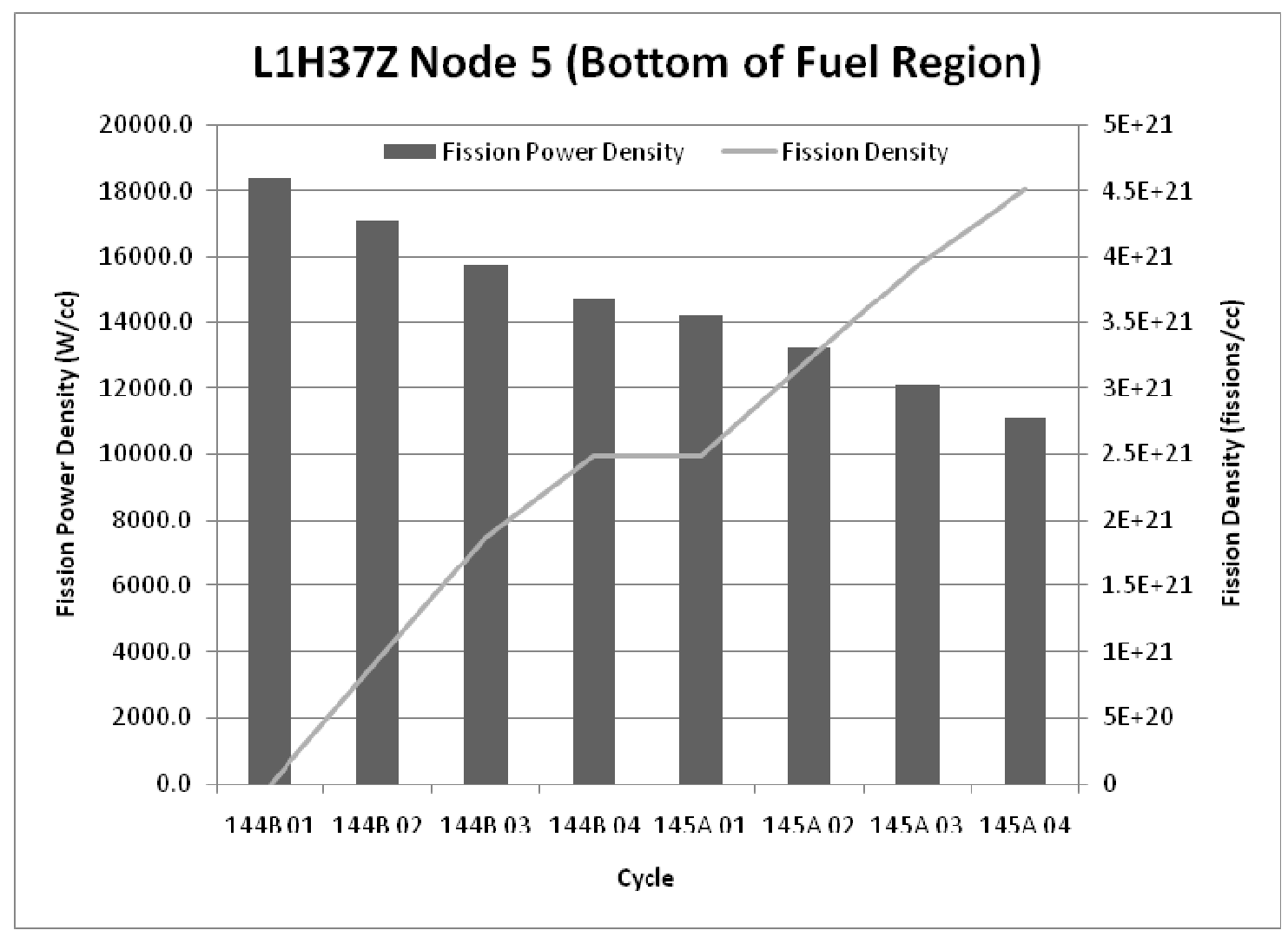




\section{A-10. Plate L1H38Z}
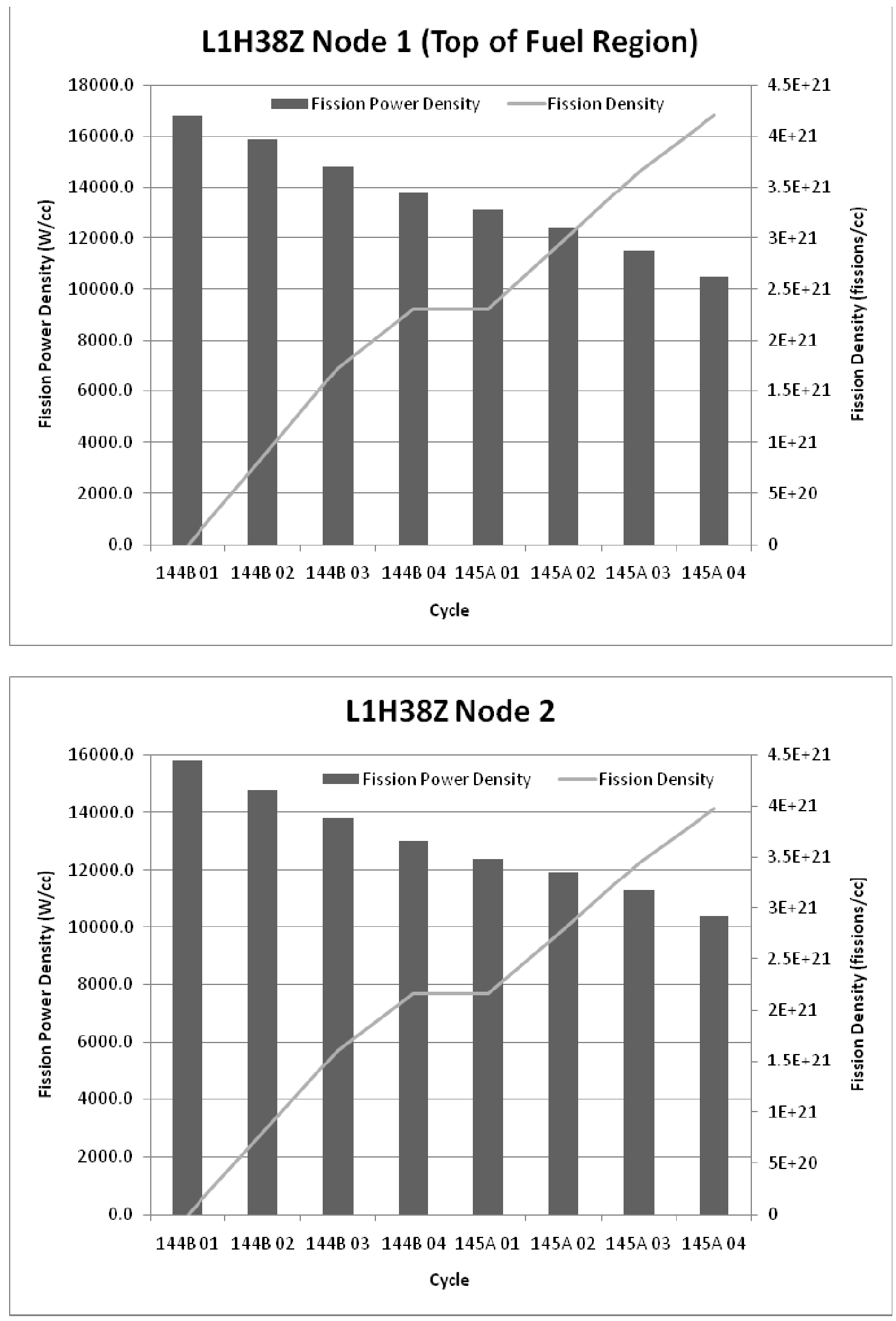

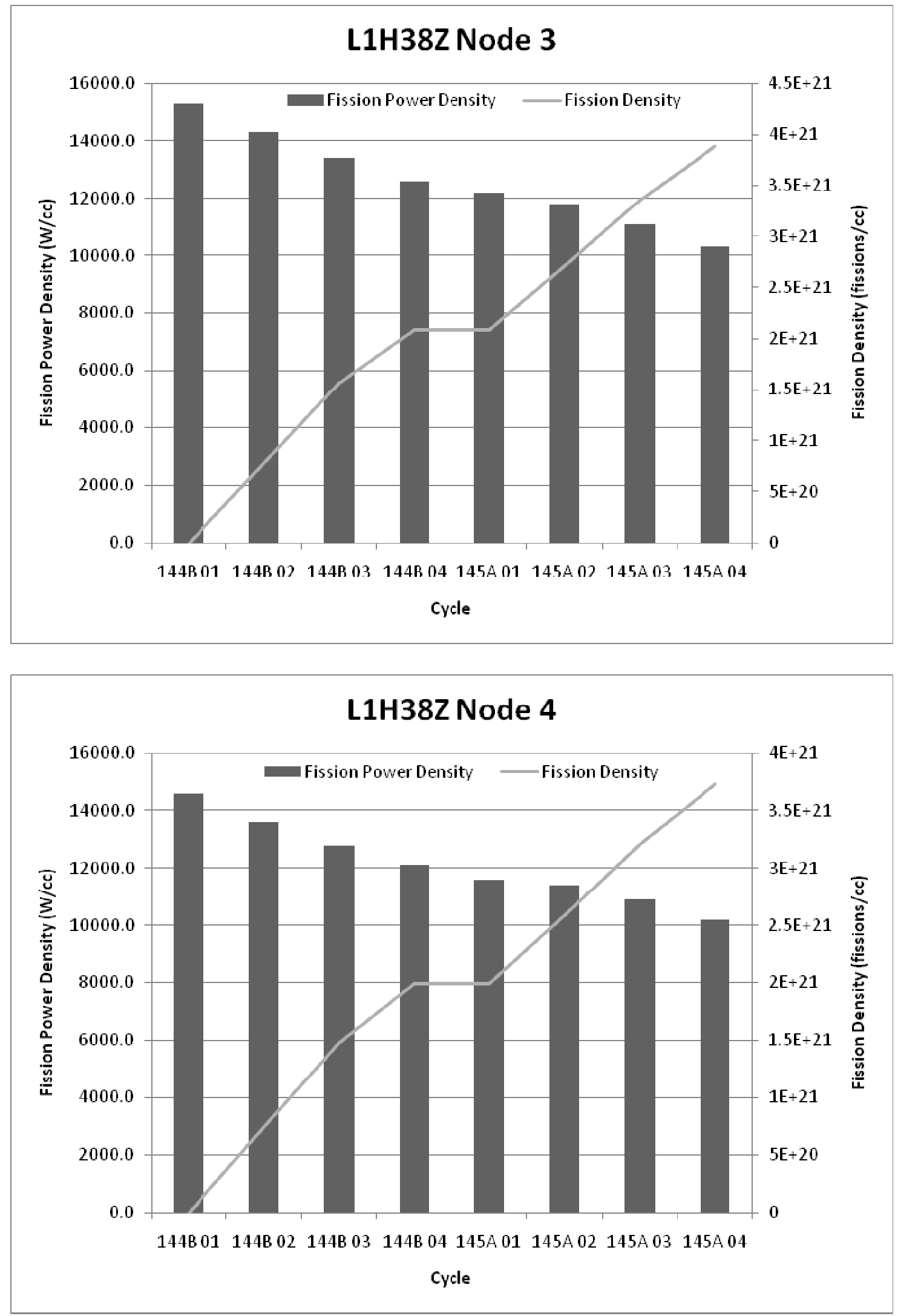


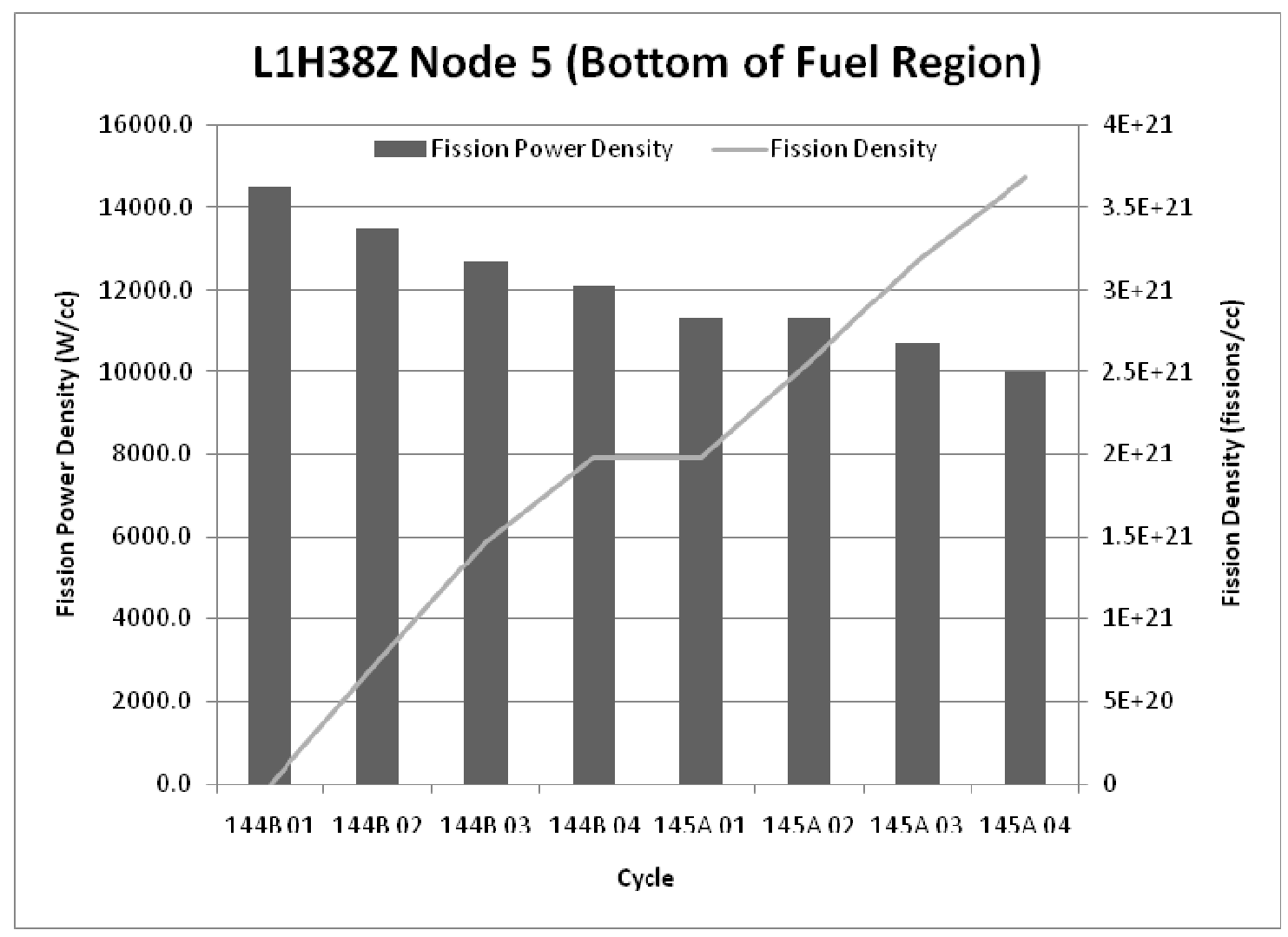




\section{A-11. Plate L1H39Z}
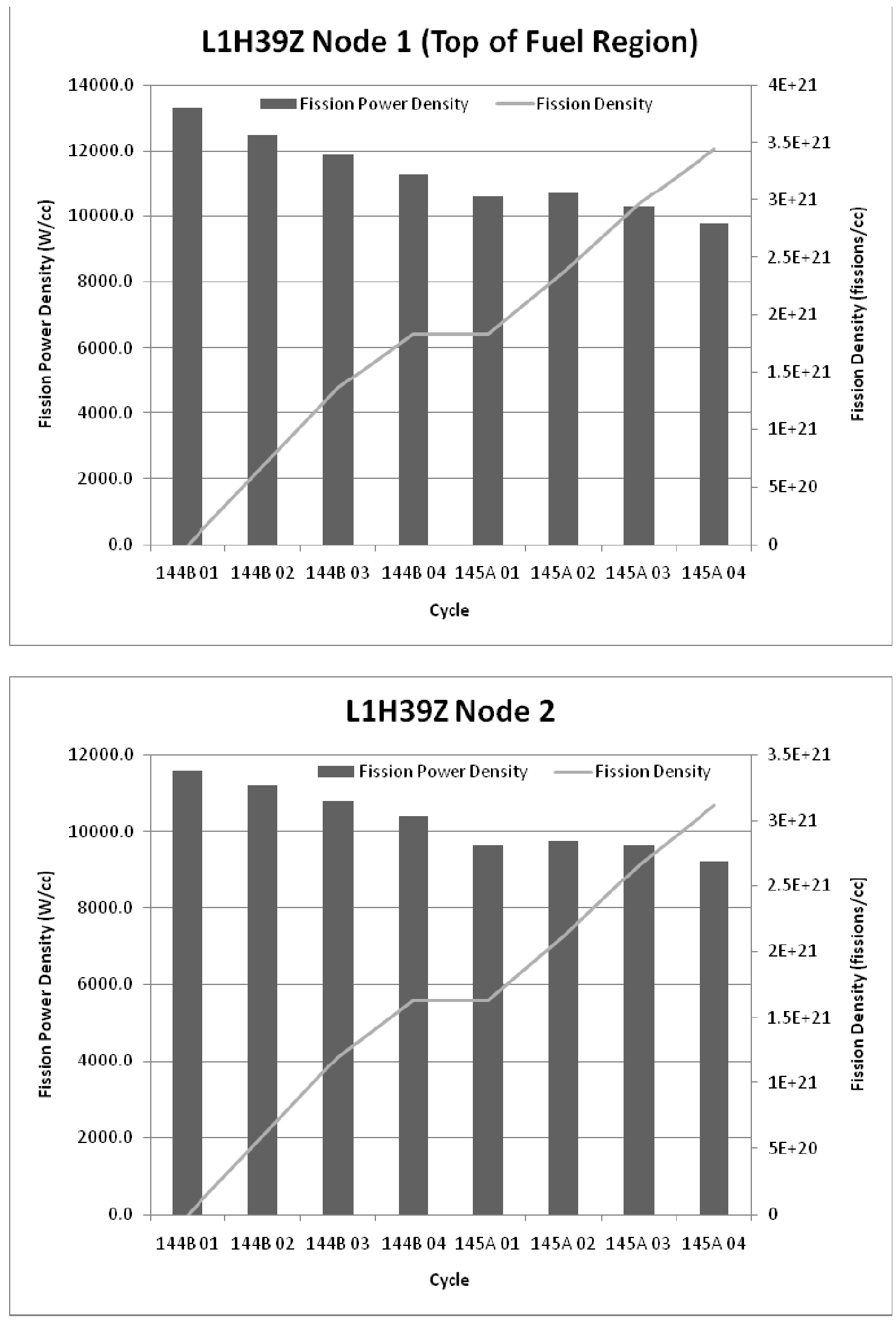

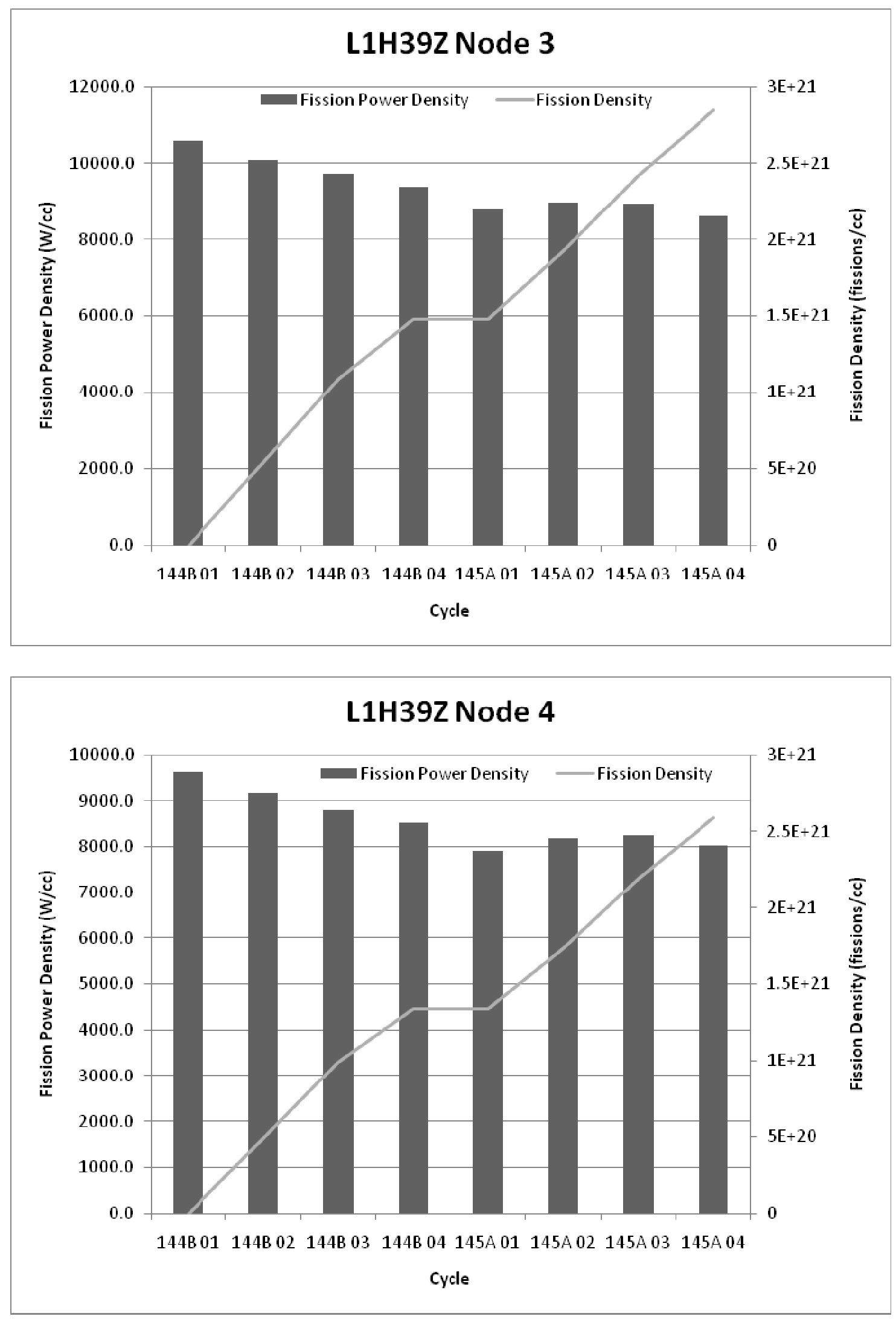


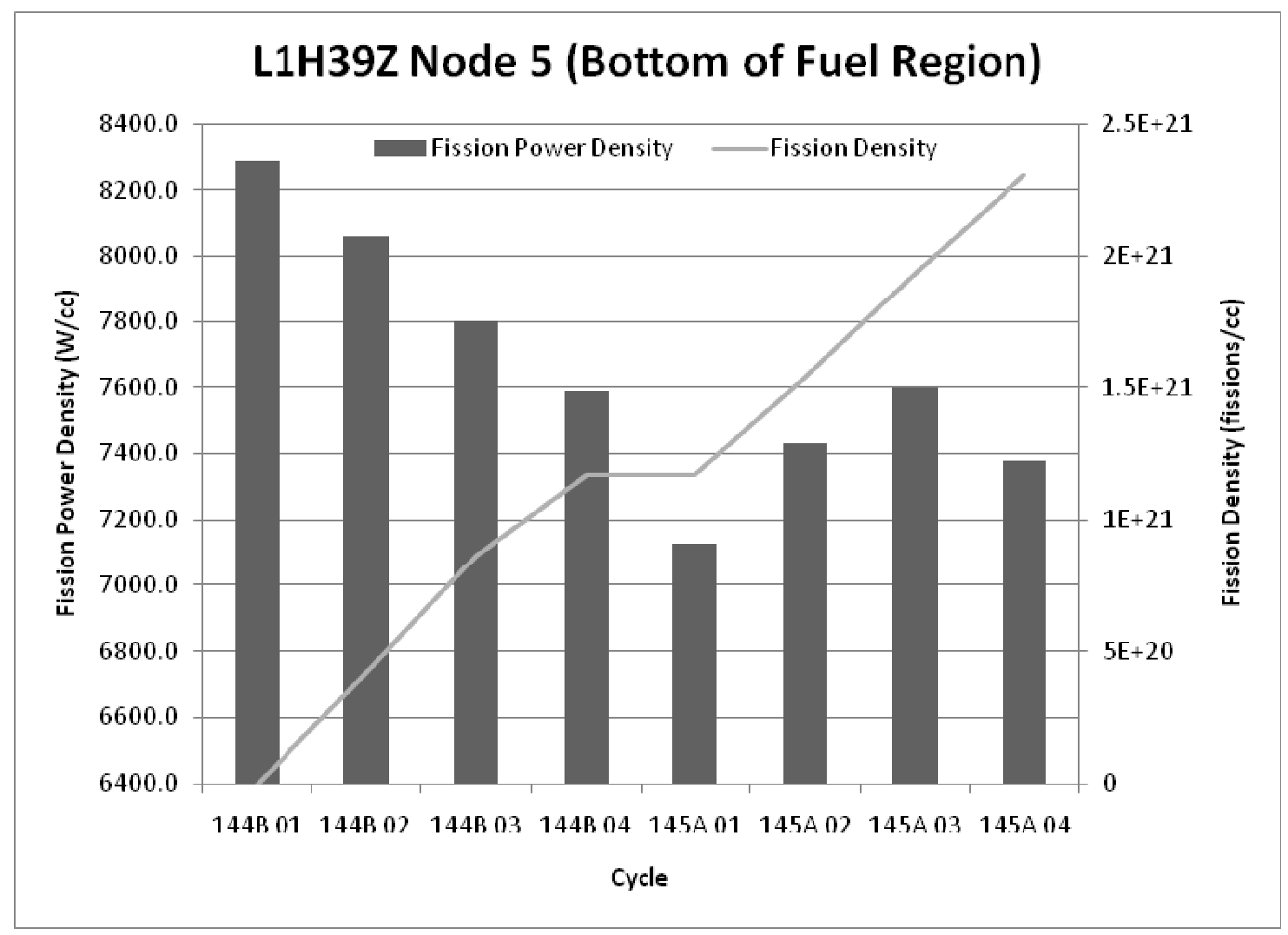


Appendix B: Plate Surface Temperatures 


\section{Appendix B}

\section{Index of Tables}

Table B - 1: Temperature $\left({ }^{\circ} \mathrm{C}\right)$ map of the east side of position A at BOC 144B (0 EFPD)................... 87

Table B - 2: Temperature $\left({ }^{\circ} \mathrm{C}\right)$ map of the west side of position A at BOC 144B (0 EFPD).................. 88

Table B - 3: Temperature $\left({ }^{\circ} \mathrm{C}\right)$ map of the east side of position B at BOC 144B (0 EFPD)................... 89

Table B - 4: Temperature $\left({ }^{\circ} \mathrm{C}\right)$ map of the west side of position B at BOC 144B (0 EFPD)...................90

Table B - 5: Temperature $\left({ }^{\circ} \mathrm{C}\right)$ map of the east side of position A at MOC1 144B (18.0 EFPD)............ 91

Table B - 6: Temperature $\left({ }^{\circ} \mathrm{C}\right)$ map of the west side of position A at MOC1 144B (18.0 EFPD)............ 92

Table B - 7: Temperature $\left({ }^{\circ} \mathrm{C}\right)$ map of the east side of position B at MOC1 144B (18.0 EFPD)............. 93

Table B - 8: Temperature $\left({ }^{\circ} \mathrm{C}\right)$ map of the west side of position B at MOC1 144B (18.0 EFPD)............. 94

Table B - 9: Temperature $\left({ }^{\circ} \mathrm{C}\right)$ map of the east side of position A at MOC2 144B (38.0 EFPD)............ 95

Table B - 10: Temperature $\left({ }^{\circ} \mathrm{C}\right)$ map of the west side of position A at MOC2 144B (38.0 EFPD).......... 96

Table B - 11: Temperature $\left({ }^{\circ} \mathrm{C}\right)$ map of the east side of position B at MOC2 144B (38.0 EFPD)........... 97

Table B - 12: Temperature $\left({ }^{\circ} \mathrm{C}\right)$ map of the west side of position B at MOC2 144B (38.0 EFPD)........... 98

Table B - 13: Temperature $\left({ }^{\circ} \mathrm{C}\right)$ map of the east side of position A at EOC 144B (51.7 EFPD)...............99

Table B - 14: Temperature $\left({ }^{\circ} \mathrm{C}\right)$ map of the west side of position A at EOC 144B (51.7 EFPD). .......... 100

Table B - 15: Temperature $\left({ }^{\circ} \mathrm{C}\right)$ map of the east side of position B at EOC 144B (51.7 EFPD)............. 101

Table B - 16: Temperature $\left({ }^{\circ} \mathrm{C}\right)$ map of the west side of position B at EOC 144B (51.7 EFPD)............ 102

Table B - 17: Temperature $\left({ }^{\circ} \mathrm{C}\right)$ map of the east side of position A at BOC 145A (total 51.7 EFPD).

Table B - 18: Temperature $\left({ }^{\circ} \mathrm{C}\right)$ map of the west side of position A at BOC 145A (total 51.7 EFPD).

Table B - 19: Temperature $\left({ }^{\circ} \mathrm{C}\right)$ map of the east side of position B at BOC 145A (total 51.7 EFPD).

Table B - 20: Temperature $\left({ }^{\circ} \mathrm{C}\right)$ map of the west side of position B at BOC 145A (total 51.7 EFPD).

Table B - 21: Temperature $\left({ }^{\circ} \mathrm{C}\right)$ map of the east side of position A at MOC1 145A (total 69.7 EFPD).

Table B - 22: Temperature $\left({ }^{\circ} \mathrm{C}\right)$ map of the west side of position A at MOC1 145A (total 69.7 EFPD).

Table B - 23: Temperature $\left({ }^{\circ} \mathrm{C}\right)$ map of the east side of position B at MOC1 145A (total 69.7 EFPD).

Table B - 24: Temperature $\left({ }^{\circ} \mathrm{C}\right)$ map of the west side of position B at MOC1 145A (total 69.7 EFPD).

Table B - 25: Temperature $\left({ }^{\circ} \mathrm{C}\right)$ map of the east side of position A at MOC2 145A (total 89.7 EFPD).

Table B - 26: Temperature $\left({ }^{\circ} \mathrm{C}\right)$ map of the west side of position A at MOC2 145A (total 89.7 EFPD).

Table B - 27: Temperature $\left({ }^{\circ} \mathrm{C}\right)$ map of the east side of position B at MOC2 145A (total 89.7 EFPD). 
Table B - 28: Temperature $\left({ }^{\circ} \mathrm{C}\right)$ map of the west side of position B at MOC2 145A (total 89.7

EFPD)

Table B - 29: Temperature $\left({ }^{\circ} \mathrm{C}\right)$ map of the east side of position A at EOC 145A (total 106.4 EFPD).

Table B - 30: Temperature $\left({ }^{\circ} \mathrm{C}\right)$ map of the west side of position A at EOC 145A (total 106.4 EFPD).

Table B - 31: Temperature $\left({ }^{\circ} \mathrm{C}\right)$ map of the east side of position B at EOC 145A (total 106.4 EFPD).

Table B - 32: Temperature $\left({ }^{\circ} \mathrm{C}\right)$ map of the west side of position B at EOC 145A (total 106.4 EFPD). 118 
Table B - 1: Temperature $\left({ }^{\circ} \mathrm{C}\right)$ map of the east side of position A at BOC 144B (0 EFPD).

\begin{tabular}{|c|c|c|c|c|c|c|c|c|c|c|c|}
\hline \multirow{2}{*}{$\begin{array}{c}\text { Plate } \\
\text { Location }\end{array}$} & \multirow{2}{*}{$\begin{array}{c}\text { Axial } \\
\text { Location (in) }\end{array}$} & \multicolumn{10}{|c|}{ Width of Plate (inches) } \\
\hline & & 0.00 & 0.08 & 0.16 & 0.25 & 0.50 & 1.00 & 1.50 & 2.00 & 2.08 & 2.16 \\
\hline \multirow{9}{*}{ A-1 } & 0.0 & 51.67 & 51.77 & 51.76 & 51.76 & 51.77 & 51.77 & 51.76 & 51.76 & 51.77 & 51.67 \\
\hline & 1.0 & 52.50 & 53.98 & 77.10 & 79.61 & 79.68 & 79.68 & 79.61 & 67.20 & 53.98 & 52.48 \\
\hline & 2.0 & 52.67 & 54.39 & 81.11 & 83.94 & 84.03 & 84.03 & 83.94 & 69.77 & 54.39 & 52.63 \\
\hline & 3.0 & 52.83 & 54.62 & 81.71 & 84.64 & 84.75 & 84.75 & 84.64 & 70.22 & 54.61 & 52.79 \\
\hline & 4.0 & 53.03 & 55.00 & 84.92 & 88.09 & 88.22 & 88.22 & 88.09 & 72.31 & 54.99 & 53.00 \\
\hline & 5.0 & 53.21 & 55.43 & 88.59 & 92.05 & 92.20 & 92.20 & 92.05 & 74.71 & 55.43 & 53.17 \\
\hline & 6.0 & 53.43 & 55.99 & 93.23 & 97.04 & 97.20 & 97.20 & 97.04 & 77.77 & 55.98 & 53.37 \\
\hline & 7.0 & 53.38 & 53.33 & 56.71 & 57.76 & 57.94 & 57.94 & 57.76 & 55.25 & 53.31 & 53.31 \\
\hline & 7.5 & 53.38 & 52.72 & 53.17 & 53.44 & 53.59 & 53.59 & 53.44 & 52.93 & 52.70 & 53.30 \\
\hline \multirow{9}{*}{ A-2 } & 7.5 & 53.38 & 52.72 & 53.17 & 53.44 & 53.59 & 53.59 & 53.44 & 52.93 & 52.70 & 53.30 \\
\hline & 8.0 & 53.54 & 53.56 & 57.52 & 58.72 & 58.91 & 58.91 & 58.72 & 55.81 & 53.54 & 53.46 \\
\hline & 9.0 & 53.98 & 57.02 & 100.71 & 105.40 & 105.64 & 105.64 & 105.40 & 82.77 & 57.01 & 53.95 \\
\hline & 10.0 & 54.17 & 57.27 & 101.76 & 106.56 & 106.82 & 106.82 & 106.56 & 83.48 & 57.26 & 54.12 \\
\hline & 11.0 & 54.39 & 57.72 & 104.74 & 109.81 & 110.10 & 110.10 & 109.81 & 85.38 & 57.71 & 54.32 \\
\hline & 12.0 & 54.59 & 58.08 & 107.18 & 112.44 & 112.75 & 112.75 & 112.44 & 86.93 & 58.07 & 54.50 \\
\hline & 13.0 & 54.78 & 58.37 & 107.77 & 113.13 & 113.47 & 113.47 & 113.13 & 87.38 & 58.36 & 54.71 \\
\hline & 14.0 & 54.95 & 58.68 & 111.36 & 116.93 & 117.28 & 117.28 & 116.93 & 89.57 & 58.67 & 54.87 \\
\hline & 15.0 & 54.76 & 54.14 & 55.15 & 55.75 & 56.11 & 56.11 & 55.75 & 54.66 & 54.12 & 54.66 \\
\hline \multirow{9}{*}{ A-3 } & 15.0 & 54.76 & 54.14 & 55.15 & 55.75 & 56.11 & 56.11 & 55.75 & 54.66 & 54.12 & 54.66 \\
\hline & 16.0 & 55.31 & 58.79 & 108.00 & 113.36 & 113.72 & 113.72 & 113.36 & 87.66 & 58.77 & 55.19 \\
\hline & 17.0 & 55.50 & 58.97 & 107.39 & 112.72 & 113.11 & 113.11 & 112.72 & 87.35 & 58.95 & 55.43 \\
\hline & 18.0 & 55.68 & 59.18 & 107.62 & 112.99 & 113.41 & 113.41 & 112.99 & 87.56 & 59.17 & 55.62 \\
\hline & 19.0 & 55.88 & 59.50 & 108.84 & 114.30 & 114.75 & 114.75 & 114.30 & 88.38 & 59.49 & 55.81 \\
\hline & 20.0 & 56.06 & 59.75 & 109.51 & 115.04 & 115.50 & 115.50 & 115.04 & 88.86 & 59.73 & 55.95 \\
\hline & 21.0 & 56.26 & 60.15 & 111.60 & 117.30 & 117.78 & 117.78 & 117.30 & 90.22 & 60.13 & 56.16 \\
\hline & 22.0 & 56.13 & 56.62 & 62.01 & 63.91 & 64.46 & 64.46 & 63.91 & 59.69 & 56.59 & 56.04 \\
\hline & 22.5 & 56.09 & 55.75 & 57.08 & 57.95 & 58.48 & 58.48 & 57.95 & 56.46 & 55.74 & 56.03 \\
\hline \multirow{9}{*}{ A-4 } & 22.5 & 56.09 & 55.75 & 57.08 & 57.95 & 58.48 & 58.48 & 57.95 & 56.46 & 55.74 & 56.03 \\
\hline & 23.0 & 56.28 & 56.85 & 62.57 & 64.55 & 65.11 & 65.11 & 64.55 & 60.11 & 56.82 & 56.18 \\
\hline & 24.0 & 56.75 & 61.12 & 117.00 & 123.10 & 123.61 & 123.61 & 123.10 & 93.71 & 61.10 & 56.63 \\
\hline & 25.0 & 56.89 & 61.15 & 114.78 & 120.73 & 121.26 & 121.26 & 120.73 & 92.42 & 61.13 & 56.78 \\
\hline & 26.0 & 57.07 & 61.42 & 114.98 & 120.96 & 121.52 & 121.52 & 120.96 & 92.63 & 61.40 & 56.99 \\
\hline & 27.0 & 57.21 & 61.62 & 114.92 & 120.90 & 121.47 & 121.47 & 120.90 & 92.67 & 61.61 & 57.15 \\
\hline & 28.0 & 57.36 & 61.86 & 115.19 & 121.20 & 121.80 & 121.80 & 121.20 & 92.92 & 61.85 & 57.28 \\
\hline & 29.0 & 57.45 & 62.03 & 116.49 & 122.53 & 123.13 & 123.13 & 122.53 & 93.72 & 62.01 & 57.34 \\
\hline & 30.0 & 57.23 & 57.56 & 59.29 & 60.43 & 61.13 & 61.13 & 60.43 & 58.51 & 57.53 & 57.11 \\
\hline \multirow{9}{*}{ A-5 } & 30.0 & 57.23 & 57.56 & 59.29 & 60.43 & 61.13 & 61.13 & 60.43 & 58.51 & 57.53 & 57.11 \\
\hline & 31.0 & 57.70 & 62.43 & 116.06 & 122.09 & 122.69 & 122.69 & 122.09 & 93.64 & 62.42 & 57.61 \\
\hline & 32.0 & 57.83 & 62.45 & 113.06 & 118.90 & 119.53 & 119.53 & 118.90 & 91.90 & 62.44 & 57.73 \\
\hline & 33.0 & 57.93 & 62.60 & 112.98 & 118.78 & 119.43 & 119.43 & 118.78 & 91.89 & 62.58 & 57.81 \\
\hline & 34.0 & 58.04 & 62.72 & 111.58 & 117.28 & 117.95 & 117.95 & 117.28 & 91.13 & 62.70 & 57.93 \\
\hline & 35.0 & 58.13 & 62.75 & 109.61 & 115.16 & 115.84 & 115.84 & 115.16 & 89.99 & 62.73 & 58.04 \\
\hline & 36.0 & 58.23 & 62.92 & 109.15 & 114.66 & 115.35 & 115.35 & 114.66 & 89.79 & 62.91 & 58.16 \\
\hline & 37.0 & 58.06 & 59.89 & 65.25 & 67.38 & 68.18 & 68.18 & 67.38 & 62.98 & 59.87 & 57.98 \\
\hline & 37.5 & 58.00 & 59.18 & 61.05 & 62.30 & 63.10 & 63.10 & 62.30 & 60.24 & 59.16 & 57.89 \\
\hline \multirow{9}{*}{ A-6 } & 37.5 & 58.00 & 59.18 & 61.05 & 62.30 & 63.10 & 63.10 & 62.30 & 60.24 & 59.16 & 57.89 \\
\hline & 38.0 & 58.11 & 59.91 & 64.77 & 66.77 & 67.56 & 67.56 & 66.77 & 62.72 & 59.89 & 58.00 \\
\hline & 39.0 & 58.38 & 62.95 & 104.46 & 109.54 & 110.23 & 110.23 & 109.54 & 87.09 & 62.93 & 58.27 \\
\hline & 40.0 & 58.41 & 62.74 & 99.70 & 104.40 & 105.10 & 105.10 & 104.40 & 84.26 & 62.72 & 58.32 \\
\hline & 41.0 & 58.44 & 62.65 & 96.44 & 100.86 & 101.57 & 101.57 & 100.86 & 82.34 & 62.64 & 58.35 \\
\hline & 42.0 & 58.47 & 62.60 & 93.71 & 97.90 & 98.61 & 98.61 & 97.90 & 80.74 & 62.58 & 58.36 \\
\hline & 43.0 & 58.49 & 62.64 & 93.39 & 97.49 & 98.20 & 98.20 & 97.49 & 80.57 & 62.63 & 58.39 \\
\hline & 44.0 & 58.46 & 62.45 & 89.81 & 93.44 & 94.14 & 94.14 & 93.44 & 78.35 & 62.44 & 58.40 \\
\hline & 45.0 & 58.29 & 60.33 & 62.10 & 63.32 & 64.11 & 64.11 & 63.32 & 61.37 & 60.33 & 58.26 \\
\hline
\end{tabular}


Table B - 2: Temperature $\left({ }^{\circ} \mathrm{C}\right)$ map of the west side of position A at BOC 144B (0 EFPD).

\begin{tabular}{|c|c|c|c|c|c|c|c|c|c|c|c|}
\hline \multirow{2}{*}{$\begin{array}{c}\text { Plate } \\
\text { Location }\end{array}$} & \multirow{2}{*}{$\begin{array}{c}\text { Axial } \\
\text { Location (in) }\end{array}$} & \multicolumn{10}{|c|}{ Width of Plate (inches) } \\
\hline & & 0.00 & 0.08 & 0.16 & 0.25 & 0.50 & 1.00 & 1.50 & 2.00 & 2.08 & 2.16 \\
\hline \multirow{9}{*}{ A-1 } & 0.0 & 51.67 & 51.77 & 51.76 & 51.76 & 51.77 & 51.77 & 51.76 & 51.76 & 51.77 & 51.67 \\
\hline & 1.0 & 52.62 & 53.98 & 77.11 & 79.62 & 79.70 & 79.70 & 79.62 & 67.21 & 53.97 & 52.58 \\
\hline & 2.0 & 52.81 & 54.40 & 81.15 & 83.98 & 84.08 & 84.08 & 83.98 & 69.79 & 54.40 & 52.74 \\
\hline & 3.0 & 52.99 & 54.65 & 81.77 & 84.72 & 84.84 & 84.84 & 84.72 & 70.26 & 54.64 & 52.90 \\
\hline & 4.0 & 53.22 & 55.05 & 85.03 & 88.23 & 88.37 & 88.37 & 88.23 & 72.39 & 55.05 & 53.14 \\
\hline & 5.0 & 53.42 & 55.51 & 88.75 & 92.24 & 92.41 & 92.41 & 92.24 & 74.83 & 55.51 & 53.33 \\
\hline & 6.0 & 53.66 & 56.11 & 93.43 & 97.28 & 97.48 & 97.48 & 97.28 & 77.93 & 56.10 & 53.54 \\
\hline & 7.0 & 53.58 & 53.47 & 56.93 & 58.03 & 58.24 & 58.24 & 58.03 & 55.44 & 53.46 & 53.44 \\
\hline & 7.5 & 53.56 & 52.87 & 53.42 & 53.74 & 53.92 & 53.92 & 53.73 & 53.14 & 52.86 & 53.41 \\
\hline \multirow{9}{*}{ A-2 } & 7.5 & 53.56 & 52.87 & 53.42 & 53.74 & 53.92 & 53.92 & 53.73 & 53.14 & 52.86 & 53.41 \\
\hline & 8.0 & 53.76 & 53.73 & 57.77 & 59.03 & 59.25 & 59.25 & 59.03 & 56.03 & 53.72 & 53.60 \\
\hline & 9.0 & 54.27 & 57.21 & 101.09 & 105.86 & 106.15 & 106.15 & 105.86 & 83.03 & 57.20 & 54.20 \\
\hline & 10.0 & 54.47 & 57.50 & 102.20 & 107.09 & 107.42 & 107.42 & 107.09 & 83.77 & 57.49 & 54.37 \\
\hline & 11.0 & 54.72 & 58.00 & 105.27 & 110.43 & 110.79 & 110.79 & 110.43 & 85.73 & 57.98 & 54.57 \\
\hline & 12.0 & 54.94 & 58.40 & 107.78 & 113.15 & 113.55 & 113.55 & 113.15 & 87.33 & 58.38 & 54.76 \\
\hline & 13.0 & 55.15 & 58.74 & 108.45 & 113.94 & 114.37 & 114.37 & 113.93 & 87.84 & 58.73 & 55.00 \\
\hline & 14.0 & 55.33 & 59.10 & 112.13 & 117.84 & 118.29 & 118.29 & 117.84 & 90.09 & 59.09 & 55.16 \\
\hline & 15.0 & 55.09 & 54.62 & 55.83 & 56.57 & 57.02 & 57.02 & 56.57 & 55.26 & 54.60 & 54.89 \\
\hline \multirow{9}{*}{ A-3 } & 15.0 & 55.09 & 54.62 & 55.83 & 56.57 & 57.02 & 57.02 & 56.57 & 55.26 & 54.60 & 54.89 \\
\hline & 16.0 & 55.73 & 59.30 & 108.92 & 114.43 & 114.92 & 114.92 & 114.43 & 88.28 & 59.28 & 55.49 \\
\hline & 17.0 & 55.95 & 59.52 & 108.38 & 113.88 & 114.41 & 114.41 & 113.88 & 88.03 & 59.50 & 55.80 \\
\hline & 18.0 & 56.14 & 59.79 & 108.70 & 114.25 & 114.81 & 114.81 & 114.25 & 88.30 & 59.77 & 56.03 \\
\hline & 19.0 & 56.37 & 60.18 & 110.02 & 115.69 & 116.29 & 116.29 & 115.69 & 89.19 & 60.16 & 56.22 \\
\hline & 20.0 & 56.56 & 60.50 & 110.79 & 116.54 & 117.17 & 117.17 & 116.54 & 89.74 & 60.47 & 56.34 \\
\hline & 21.0 & 56.79 & 60.97 & 112.99 & 118.92 & 119.58 & 119.58 & 118.92 & 91.18 & 60.94 & 56.57 \\
\hline & 22.0 & 56.61 & 57.50 & 63.21 & 65.32 & 66.03 & 66.03 & 65.31 & 60.76 & 57.48 & 56.40 \\
\hline & 22.5 & 56.54 & 56.66 & 58.33 & 59.42 & 60.11 & 60.11 & 59.42 & 57.56 & 56.65 & 56.44 \\
\hline \multirow{9}{*}{ A-4 } & 22.5 & 56.54 & 56.66 & 58.33 & 59.42 & 60.11 & 60.11 & 59.42 & 57.56 & 56.65 & 256.44 \\
\hline & 23.0 & 56.76 & 57.78 & 63.82 & 66.01 & 66.73 & 66.73 & 66.01 & 61.23 & 57.76 & 56.56 \\
\hline & 24.0 & 57.32 & 62.10 & 118.61 & 124.97 & 125.67 & 125.67 & 124.97 & 94.85 & 62.07 & 57.06 \\
\hline & 25.0 & 57.47 & 62.19 & 116.49 & 122.71 & 123.45 & 123.45 & 122.71 & 93.60 & 62.16 & 57.23 \\
\hline & 26.0 & 57.67 & 62.53 & 116.81 & 123.07 & 123.84 & 123.84 & 123.06 & 93.92 & 62.50 & 57.50 \\
\hline & 27.0 & 57.83 & 62.80 & 116.84 & 123.12 & 123.92 & 123.92 & 123.11 & 94.05 & 62.78 & 57.70 \\
\hline & 28.0 & 57.99 & 63.12 & 117.22 & 123.54 & 124.37 & 124.36 & 123.54 & 94.40 & 63.09 & 57.81 \\
\hline & 29.0 & 58.12 & 63.46 & 118.59 & 125.01 & 125.85 & 125.85 & 125.01 & 95.36 & 63.43 & 57.86 \\
\hline & 30.0 & 57.80 & 58.97 & 61.14 & 62.57 & 63.49 & 63.49 & 62.57 & 60.17 & 58.94 & 57.54 \\
\hline \multirow{9}{*}{ A-5 } & 30.0 & 57.80 & 58.97 & 61.14 & 62.57 & 63.50 & 63.49 & 62.57 & 60.17 & 58.94 & 57.54 \\
\hline & 31.0 & 58.36 & 63.88 & 118.33 & 124.70 & 125.55 & 125.55 & 124.70 & 95.38 & 63.85 & 58.15 \\
\hline & 32.0 & 58.50 & 63.96 & 115.44 & 121.61 & 122.50 & 122.50 & 121.61 & 93.66 & 63.93 & 58.27 \\
\hline & 33.0 & 58.61 & 64.16 & 115.43 & 121.58 & 122.49 & 122.49 & 121.58 & 93.74 & 64.13 & 58.35 \\
\hline & 34.0 & 58.73 & 64.36 & 114.15 & 120.21 & 121.15 & 121.15 & 120.21 & 93.05 & 64.32 & 58.48 \\
\hline & 35.0 & 58.82 & 64.45 & 112.29 & 118.21 & 119.16 & 119.16 & 118.20 & 91.97 & 64.42 & 58.64 \\
\hline & 36.0 & 58.93 & 64.70 & 111.93 & 117.81 & 118.79 & 118.79 & 117.81 & 91.86 & 64.67 & 58.78 \\
\hline & 37.0 & 58.72 & 61.75 & 67.56 & 70.00 & 71.04 & 71.04 & 69.99 & 65.10 & 61.72 & 58.55 \\
\hline & 37.5 & 58.65 & 61.07 & 63.42 & 65.01 & 66.05 & 66.05 & 65.01 & 62.40 & 61.04 & 58.40 \\
\hline \multirow{9}{*}{ A-6 } & $\begin{array}{l}37.5 \\
\end{array}$ & 58.65 & 61.07 & 63.42 & 65.01 & 66.05 & 66.05 & 65.01 & 62.40 & 61.04 & 58.40 \\
\hline & 38.0 & 58.77 & 61.81 & 67.13 & 69.44 & 70.47 & 70.47 & 69.44 & 64.88 & 61.78 & 58.53 \\
\hline & 39.0 & 59.09 & 64.89 & 107.47 & 112.93 & 113.90 & 113.90 & 112.93 & 89.28 & 64.85 & 58.85 \\
\hline & 40.0 & 59.12 & 64.73 & 102.81 & 107.90 & 108.89 & 108.89 & 107.89 & 86.52 & 64.70 & 58.90 \\
\hline & 41.0 & 59.16 & 64.71 & 99.66 & 104.48 & 105.48 & 105.48 & 104.47 & 84.69 & 64.68 & 58.95 \\
\hline & 42.0 & 59.19 & 64.71 & 97.02 & 101.62 & 102.63 & 102.63 & 101.61 & 83.16 & 64.68 & 58.94 \\
\hline & 43.0 & 59.20 & 64.81 & 96.77 & 101.28 & 102.29 & 102.29 & 101.27 & 83.05 & 64.77 & 58.98 \\
\hline & 44.0 & 59.17 & 64.67 & 93.18 & 97.33 & 98.34 & 98.34 & 97.33 & 80.91 & 64.64 & 59.03 \\
\hline & 45.0 & 58.96 & 62.60 & 64.84 & 66.39 & 67.43 & 67.43 & 66.39 & 63.90 & 62.57 & 58.87 \\
\hline
\end{tabular}


Table B - 3: Temperature $\left({ }^{\circ} \mathrm{C}\right)$ map of the east side of position B at BOC 144B (0 EFPD).

\begin{tabular}{|c|c|c|c|c|c|c|c|c|c|c|c|}
\hline \multirow{2}{*}{$\begin{array}{c}\text { Plate } \\
\text { Location }\end{array}$} & \multirow{2}{*}{$\begin{array}{c}\text { Axial } \\
\text { Location (in) }\end{array}$} & \multicolumn{10}{|c|}{ Width of Plate (inches) } \\
\hline & & 0.00 & 0.08 & 0.16 & 0.25 & 0.50 & 1.00 & 1.50 & 2.00 & 2.08 & 2.16 \\
\hline \multirow{9}{*}{ B-1 } & 0.0 & 51.67 & 51.79 & 51.76 & 51.76 & 51.77 & 51.77 & 51.76 & 51.76 & 51.79 & 51.67 \\
\hline & 1.0 & 52.96 & 54.04 & 76.98 & 79.48 & 79.55 & 79.55 & 79.48 & 76.98 & 54.01 & 52.86 \\
\hline & 2.0 & 53.19 & 54.45 & 80.70 & 83.49 & 83.59 & 83.59 & 83.49 & 80.69 & 54.40 & 52.94 \\
\hline & 3.0 & 53.41 & 54.70 & 81.32 & 84.23 & 84.35 & 84.35 & 84.23 & 81.32 & 54.64 & 53.04 \\
\hline & 4.0 & 53.67 & 55.14 & 84.89 & 88.07 & 88.22 & 88.22 & 88.07 & 84.88 & 55.09 & 53.33 \\
\hline & 5.0 & 53.91 & 55.60 & 88.45 & 91.91 & 92.08 & 92.08 & 91.91 & 88.45 & 55.53 & 53.50 \\
\hline & 6.0 & 54.20 & 56.22 & 93.29 & 97.13 & 97.32 & 97.32 & 97.13 & 93.29 & 56.14 & 53.71 \\
\hline & 7.0 & 54.15 & 53.61 & 56.94 & 58.02 & 58.23 & 58.23 & 58.02 & 56.93 & 53.52 & 53.63 \\
\hline & 7.5 & 54.15 & 53.02 & 53.43 & 53.75 & 53.93 & 53.93 & 53.74 & 53.43 & 52.93 & 53.61 \\
\hline \multirow{9}{*}{ B-2 } & 7.5 & 54.15 & 53.02 & 53.43 & 53.75 & 53.93 & 53.93 & 53.74 & 53.43 & 52.93 & 53.61 \\
\hline & 8.0 & 54.36 & 53.82 & 57.50 & 58.69 & 58.91 & 58.91 & 58.69 & 57.50 & 53.73 & 53.79 \\
\hline & 9.0 & 54.90 & 57.10 & 98.19 & 102.63 & 102.91 & 102.91 & 102.63 & 98.19 & 57.07 & 54.81 \\
\hline & 10.0 & 55.13 & 57.38 & 99.05 & 103.66 & 103.98 & 103.98 & 103.66 & 99.05 & 57.32 & 54.88 \\
\hline & 11.0 & 55.42 & 57.89 & 102.12 & 107.01 & 107.37 & 107.37 & 107.01 & 102.11 & 57.78 & 54.91 \\
\hline & 12.0 & 55.66 & 58.28 & 104.38 & 109.47 & 109.86 & 109.86 & 109.47 & 104.38 & 58.16 & 54.97 \\
\hline & 13.0 & 55.90 & 58.62 & 105.05 & 110.25 & 110.68 & 110.68 & 110.25 & 105.04 & 58.51 & 55.26 \\
\hline & 14.0 & 56.11 & 59.01 & 108.81 & 114.25 & 114.69 & 114.69 & 114.25 & 108.80 & 58.89 & 55.41 \\
\hline & 15.0 & 55.89 & 54.83 & 55.84 & 56.56 & 57.00 & 57.00 & 56.55 & 55.83 & 54.69 & 55.16 \\
\hline \multirow{9}{*}{ B-3 } & 15.0 & 55.89 & 54.83 & 55.84 & 56.56 & 57.00 & 57.00 & 56.55 & 55.83 & 54.69 & 55.16 \\
\hline & 16.0 & 56.58 & 59.83 & 112.58 & 118.37 & 118.85 & 118.85 & 118.37 & 112.57 & 59.70 & 55.75 \\
\hline & 17.0 & 56.81 & 60.03 & 111.64 & 117.40 & 117.92 & 117.92 & 117.39 & 111.64 & 59.95 & 56.47 \\
\hline & 18.0 & 57.02 & 60.30 & 111.93 & 117.72 & 118.28 & 118.28 & 117.72 & 111.92 & 60.25 & 56.85 \\
\hline & 19.0 & 57.26 & 60.67 & 112.91 & 118.80 & 119.39 & 119.39 & 118.80 & 112.91 & 60.57 & 56.89 \\
\hline & 20.0 & 57.47 & 60.99 & 113.73 & 119.70 & 120.32 & 120.32 & 119.70 & 113.73 & 60.85 & 56.78 \\
\hline & 21.0 & 57.72 & 61.59 & 117.26 & 123.51 & 124.16 & 124.16 & 123.51 & 117.25 & 61.44 & 56.93 \\
\hline & 22.0 & 57.53 & 57.86 & 63.76 & 65.93 & 66.64 & 66.64 & 65.93 & 63.74 & 57.71 & 56.72 \\
\hline & 22.5 & 57.47 & 56.95 & 58.48 & 59.56 & 60.25 & 60.25 & 59.56 & 58.46 & 56.90 & 57.35 \\
\hline \multirow{9}{*}{ B-4 } & 22.5 & 57.47 & 56.95 & 58.48 & 59.56 & 60.25 & 60.25 & 59.56 & 58.46 & 56.90 & 57.35 \\
\hline & 23.0 & 57.69 & 58.05 & 63.89 & 66.06 & 66.77 & 66.76 & 66.05 & 63.87 & 57.90 & 56.87 \\
\hline & 24.0 & 58.25 & 62.30 & 117.99 & 124.28 & 124.98 & 124.98 & 124.28 & 117.98 & 62.15 & 57.31 \\
\hline & 25.0 & 58.41 & 62.39 & 115.72 & 121.86 & 122.60 & 122.60 & 121.86 & 115.71 & 62.24 & 57.67 \\
\hline & 26.0 & 58.60 & 62.71 & 115.76 & 121.93 & 122.70 & 122.70 & 121.92 & 115.75 & 62.60 & 58.24 \\
\hline & 27.0 & 58.75 & 62.97 & 115.57 & 121.73 & 122.53 & 122.53 & 121.73 & 115.56 & 62.92 & 58.56 \\
\hline & 28.0 & 58.91 & 63.29 & 115.96 & 122.17 & 122.99 & 122.99 & 122.16 & 115.95 & 63.18 & 58.50 \\
\hline & 29.0 & 59.00 & 63.57 & 117.74 & 124.02 & 124.86 & 124.86 & 124.02 & 117.73 & 63.41 & 58.26 \\
\hline & 30.0 & 58.70 & 59.28 & 61.27 & 62.68 & 63.60 & 63.59 & 62.67 & 61.25 & 59.11 & 57.82 \\
\hline \multirow{9}{*}{ B-5 } & 30.0 & 58.70 & 59.28 & 61.27 & 62.68 & 63.60 & 63.59 & 62.68 & 61.25 & 59.11 & 57.82 \\
\hline & 31.0 & 59.24 & 63.77 & 113.77 & 119.77 & 120.62 & 120.62 & 119.76 & 113.76 & 63.63 & 58.50 \\
\hline & 32.0 & 59.37 & 63.89 & 111.47 & 117.32 & 118.21 & 118.21 & 117.31 & 111.46 & 63.74 & 58.53 \\
\hline & 33.0 & 59.46 & 64.11 & 111.53 & 117.36 & 118.27 & 118.26 & 117.35 & 111.51 & 63.95 & 58.54 \\
\hline & 34.0 & 59.56 & 64.33 & 110.56 & 116.33 & 117.26 & 117.26 & 116.32 & 110.55 & 64.17 & 58.81 \\
\hline & 35.0 & 59.64 & 64.44 & 108.87 & 114.49 & 115.45 & 115.45 & 114.49 & 108.85 & 64.32 & 59.23 \\
\hline & 36.0 & 59.72 & 64.71 & 108.83 & 114.46 & 115.43 & 115.42 & 114.45 & 108.82 & 64.64 & 59.43 \\
\hline & 37.0 & 59.50 & 61.98 & 67.38 & 69.73 & 70.76 & 70.75 & 69.72 & 67.35 & 61.87 & 59.17 \\
\hline & 37.5 & 59.41 & 61.36 & 63.54 & 65.10 & 66.12 & 66.12 & 65.09 & 63.51 & 61.20 & 58.59 \\
\hline \multirow{9}{*}{ B-6 } & 37.5 & 59.41 & 61.36 & 63.54 & 65.10 & 66.12 & 66.12 & 65.09 & 63.51 & 61.20 & 58.59 \\
\hline & 38.0 & 59.52 & 62.07 & 67.10 & 69.36 & 70.37 & 70.37 & 69.35 & 67.08 & 61.92 & 58.82 \\
\hline & 39.0 & 59.81 & 65.02 & 105.94 & 111.26 & 112.23 & 112.23 & 111.25 & 105.92 & 64.88 & 59.04 \\
\hline & 40.0 & 59.81 & 64.88 & 101.50 & 106.45 & 107.44 & 107.44 & 106.44 & 101.48 & 64.74 & 59.05 \\
\hline & 41.0 & 59.82 & 64.88 & 98.69 & 103.39 & 104.40 & 104.39 & 103.39 & 98.67 & 64.75 & 59.12 \\
\hline & 42.0 & 59.81 & 64.89 & 96.22 & 100.72 & 101.73 & 101.73 & 100.71 & 96.20 & 64.75 & 59.02 \\
\hline & 43.0 & 59.80 & 64.98 & 95.97 & 100.39 & 101.39 & 101.39 & 100.38 & 95.95 & 64.84 & 59.17 \\
\hline & 44.0 & 59.72 & 64.83 & 92.27 & 96.32 & 97.32 & 97.32 & 96.31 & 92.25 & 64.74 & 59.43 \\
\hline & 45.0 & 59.50 & 62.84 & 64.99 & 66.52 & 67.54 & 67.54 & 66.50 & 64.96 & 62.80 & 59.36 \\
\hline
\end{tabular}


Table B - 4: Temperature $\left({ }^{\circ} \mathrm{C}\right)$ map of the west side of position B at BOC 144B (0 EFPD).

\begin{tabular}{|c|c|c|c|c|c|c|c|c|c|c|c|}
\hline \multirow{2}{*}{$\begin{array}{c}\text { Plate } \\
\text { Location }\end{array}$} & \multirow{2}{*}{$\begin{array}{c}\text { Axial } \\
\text { Location (in) }\end{array}$} & \multicolumn{10}{|c|}{ Width of Plate (inches) } \\
\hline & & 0.00 & 0.08 & 0.16 & 0.25 & 0.50 & 1.00 & 1.50 & 2.00 & 2.08 & 2.16 \\
\hline \multirow{9}{*}{ B-1 } & 0.0 & 51.67 & 51.78 & 51.76 & 51.76 & 51.77 & 51.77 & 51.76 & 51.76 & 51.78 & 51.67 \\
\hline & 1.0 & 53.26 & 54.01 & 76.97 & 79.46 & 79.53 & 79.53 & 79.46 & 67.12 & 54.00 & 53.15 \\
\hline & 2.0 & 53.52 & 54.41 & 80.67 & 83.45 & 83.55 & 83.55 & 83.45 & 69.49 & 54.38 & 53.09 \\
\hline & 3.0 & 53.77 & 54.64 & 81.27 & 84.16 & 84.27 & 84.27 & 84.16 & 69.94 & 54.60 & 53.06 \\
\hline & 4.0 & 54.06 & 55.06 & 84.79 & 87.95 & 88.07 & 88.07 & 87.95 & 72.23 & 55.02 & 53.38 \\
\hline & 5.0 & 54.33 & 55.50 & 88.31 & 91.74 & 91.88 & 91.88 & 91.74 & 74.54 & 55.45 & 53.51 \\
\hline & 6.0 & 54.64 & 56.08 & 93.10 & 96.90 & 97.06 & 97.06 & 96.90 & 77.71 & 56.02 & 53.72 \\
\hline & 7.0 & 54.65 & 53.44 & 56.74 & 57.77 & 57.95 & 57.95 & 57.77 & 55.28 & 53.38 & 53.74 \\
\hline & 7.5 & 54.69 & 52.84 & 53.21 & 53.47 & 53.62 & 53.62 & 53.47 & 52.98 & 52.77 & 53.75 \\
\hline \multirow{9}{*}{ B-2 } & 7.5 & 54.69 & 52.84 & 53.21 & 53.47 & 53.62 & 53.62 & 53.47 & 52.98 & 52.77 & 53.75 \\
\hline & 8.0 & 54.89 & 53.64 & 57.27 & 58.41 & 58.59 & 58.59 & 58.40 & 55.67 & 53.57 & 53.89 \\
\hline & 9.0 & 55.40 & 56.90 & 97.89 & 102.20 & 102.42 & 102.42 & 102.20 & 80.97 & 56.87 & 55.44 \\
\hline & 10.0 & 55.66 & 57.14 & 98.70 & 103.16 & 103.41 & 103.41 & 103.16 & 81.56 & 57.09 & 55.38 \\
\hline & 11.0 & 55.95 & 57.60 & 101.63 & 106.42 & 106.70 & 106.70 & 106.42 & 83.48 & 57.52 & 55.09 \\
\hline & 12.0 & 56.21 & 57.95 & 103.82 & 108.78 & 109.09 & 109.08 & 108.78 & 84.88 & 57.86 & 54.89 \\
\hline & 13.0 & 56.46 & 58.25 & 104.40 & 109.47 & 109.79 & 109.79 & 109.46 & 85.33 & 58.17 & 55.20 \\
\hline & 14.0 & 56.67 & 58.58 & 108.08 & 113.36 & 113.69 & 113.69 & 113.36 & 87.58 & 58.50 & 55.31 \\
\hline & 15.0 & 56.54 & 54.35 & 55.20 & 55.77 & 56.11 & 56.10 & 55.76 & 54.73 & 54.25 & 55.24 \\
\hline \multirow{9}{*}{ B-3 } & 15.0 & 56.54 & 54.35 & 55.20 & 55.77 & 56.11 & 56.10 & 55.76 & 54.73 & 54.25 & 55.24 \\
\hline & 16.0 & 57.18 & 59.34 & 111.73 & 117.35 & 117.70 & 117.70 & 117.35 & 89.99 & 59.22 & 55.74 \\
\hline & 17.0 & 57.40 & 59.50 & 110.73 & 116.30 & 116.68 & 116.68 & 116.29 & 89.46 & 59.43 & 57.01 \\
\hline & 18.0 & 57.61 & 59.72 & 110.94 & 116.54 & 116.94 & 116.94 & 116.53 & 89.65 & 59.66 & 57.58 \\
\hline & 19.0 & 57.85 & 60.03 & 111.84 & 117.51 & 117.94 & 117.94 & 117.50 & 90.28 & 59.95 & 57.39 \\
\hline & 20.0 & 58.05 & 60.31 & 112.58 & 118.31 & 118.77 & 118.77 & 118.31 & 90.79 & 60.19 & 56.90 \\
\hline & 21.0 & 58.29 & 60.84 & 116.02 & 122.03 & 122.50 & 122.50 & 122.02 & 92.99 & 60.72 & 56.86 \\
\hline & 22.0 & 58.15 & 57.04 & 62.68 & 64.64 & 65.19 & 65.19 & 64.63 & 60.21 & 56.93 & 56.56 \\
\hline & 22.5 & 58.11 & 56.12 & 57.36 & 58.22 & 58.73 & 58.73 & 58.21 & 56.72 & 56.06 & 58.23 \\
\hline \multirow{9}{*}{ B-4 } & 22.5 & 58.11 & 56.12 & 57.36 & 58.22 & 58.73 & 58.73 & 58.21 & 56.72 & 56.06 & 58.23 \\
\hline & 23.0 & 58.30 & 57.20 & 62.77 & 64.71 & 65.26 & 65.26 & 64.71 & 60.32 & 57.08 & 56.70 \\
\hline & 24.0 & 58.80 & 61.43 & 116.55 & 122.58 & 123.07 & 123.07 & 122.57 & 93.49 & 61.29 & 57.04 \\
\hline & 25.0 & 58.93 & 61.47 & 114.19 & 120.06 & 120.58 & 120.58 & 120.05 & 92.13 & 61.33 & 57.69 \\
\hline & 26.0 & 59.09 & 61.72 & 114.14 & 120.00 & 120.55 & 120.54 & 119.99 & 92.18 & 61.62 & 58.73 \\
\hline & 27.0 & 59.24 & 61.92 & 113.85 & 119.70 & 120.26 & 120.26 & 119.69 & 92.09 & 61.84 & 59.18 \\
\hline & 28.0 & 59.37 & 62.17 & 114.14 & 120.02 & 120.60 & 120.60 & 120.01 & 92.35 & 62.07 & 58.89 \\
\hline & 29.0 & 59.44 & 62.39 & 115.83 & 121.77 & 122.35 & 122.35 & 121.76 & 93.39 & 62.25 & 58.18 \\
\hline & 30.0 & 59.18 & 58.02 & 59.62 & 60.72 & 61.40 & 61.40 & 60.70 & 58.82 & 57.87 & 57.59 \\
\hline \multirow{9}{*}{ B-5 } & 30.0 & 59.18 & 58.02 & 59.62 & 60.72 & 61.40 & 61.40 & 60.70 & 58.82 & 57.87 & 57.59 \\
\hline & 31.0 & 59.63 & 62.49 & 111.71 & 117.35 & 117.93 & 117.93 & 117.33 & 91.04 & 62.36 & 58.23 \\
\hline & 32.0 & 59.72 & 62.55 & 109.31 & 114.80 & 115.41 & 115.40 & 114.78 & 89.67 & 62.42 & 58.09 \\
\hline & 33.0 & 59.78 & 62.72 & 109.29 & 114.74 & 115.37 & 115.36 & 114.73 & 89.71 & 62.57 & 58.03 \\
\hline & 34.0 & 59.84 & 62.87 & 108.21 & 113.59 & 114.23 & 114.22 & 113.57 & 89.14 & 62.72 & 58.56 \\
\hline & 35.0 & 59.88 & 62.91 & 106.41 & 111.64 & 112.29 & 112.29 & 111.63 & 88.12 & 62.80 & 59.39 \\
\hline & 36.0 & 59.92 & 63.12 & 106.28 & 111.49 & 112.15 & 112.15 & 111.48 & 88.13 & 63.02 & 59.62 \\
\hline & 37.0 & 59.70 & 60.31 & 65.27 & 67.28 & 68.05 & 68.04 & 67.26 & 63.10 & 60.20 & 59.41 \\
\hline & 37.5 & 59.62 & 59.66 & 61.38 & 62.57 & 63.33 & 63.32 & 62.55 & 60.56 & 59.51 & 58.14 \\
\hline \multirow{9}{*}{ B-6 } & 37.5 & 59.62 & 59.66 & 61.38 & 62.57 & 63.33 & 63.32 & 62.55 & 60.56 & 59.51 & 58.14 \\
\hline & 38.0 & 59.69 & 60.36 & 64.95 & 66.86 & 67.62 & 67.61 & 66.85 & 62.94 & 60.22 & 58.51 \\
\hline & 39.0 & 59.89 & 63.29 & 103.19 & 108.08 & 108.73 & 108.73 & 108.07 & 86.40 & 63.14 & 58.50 \\
\hline & $\begin{array}{l}40.0 \\
\end{array}$ & 59.86 & 63.10 & 98.64 & 103.17 & 103.84 & 103.83 & 103.15 & 83.71 & 62.96 & 58.37 \\
\hline & 41.0 & 59.82 & 63.04 & 95.73 & 100.00 & 100.68 & 100.67 & 99.98 & 82.00 & 62.90 & 58.53 \\
\hline & 42.0 & 59.77 & 63.00 & 93.18 & 97.24 & 97.91 & 97.91 & 97.22 & 80.51 & 62.85 & 58.30 \\
\hline & 43.0 & 59.72 & 63.05 & 92.87 & 96.84 & 97.52 & 97.51 & 96.82 & 80.35 & 62.90 & 58.67 \\
\hline & 44.0 & 59.60 & 62.85 & 89.18 & 92.67 & 93.33 & 93.33 & 92.64 & 78.07 & 62.74 & 59.19 \\
\hline & 45.0 & 59.38 & 60.82 & 62.49 & 63.64 & 64.39 & 64.38 & 63.62 & 61.74 & 60.73 & 59.23 \\
\hline
\end{tabular}


Table B - 5: Temperature $\left({ }^{\circ} \mathrm{C}\right)$ map of the east side of position A at MOC1 144B (18.0 EFPD).

\begin{tabular}{|c|c|c|c|c|c|c|c|c|c|c|c|}
\hline \multirow{2}{*}{$\begin{array}{c}\text { Plate } \\
\text { Location }\end{array}$} & \multirow{2}{*}{$\begin{array}{c}\text { Axial } \\
\text { Location (in) }\end{array}$} & \multicolumn{10}{|c|}{ Width of Plate (inches) } \\
\hline & & 0.00 & 0.08 & 0.16 & 0.25 & 0.50 & 1.00 & 1.50 & 2.00 & 2.08 & 2.16 \\
\hline \multirow{9}{*}{ A-1 } & 0.0 & 51.67 & 51.77 & 51.76 & 51.76 & 51.77 & 51.77 & 51.76 & 51.76 & 51.77 & 51.67 \\
\hline & 1.0 & 52.49 & 53.93 & 76.50 & 78.96 & 79.03 & 79.03 & 78.96 & 66.83 & 53.92 & 52.48 \\
\hline & 2.0 & 52.66 & 54.29 & 79.93 & 82.66 & 82.75 & 82.75 & 82.66 & 69.02 & 54.28 & 52.63 \\
\hline & 3.0 & 52.82 & 54.51 & 80.50 & 83.33 & 83.44 & 83.44 & 83.33 & 69.44 & 54.50 & 52.78 \\
\hline & 4.0 & 53.02 & 54.90 & 83.92 & 87.02 & 87.14 & 87.14 & 87.02 & 71.66 & 54.89 & 52.98 \\
\hline & 5.0 & 53.20 & 55.27 & 86.91 & 90.25 & 90.39 & 90.39 & 90.25 & 73.62 & 55.26 & 53.15 \\
\hline & 6.0 & 53.41 & 55.80 & 91.34 & 95.01 & 95.17 & 95.17 & 95.01 & 76.54 & 55.79 & 53.35 \\
\hline & 7.0 & 53.37 & 53.27 & 56.48 & 57.48 & 57.64 & 57.64 & 57.48 & 55.09 & 53.25 & 53.30 \\
\hline & 7.5 & 53.36 & 52.69 & 53.11 & 53.37 & 53.51 & 53.51 & 53.37 & 52.89 & 52.67 & 53.29 \\
\hline \multirow{9}{*}{ A-2 } & 7.5 & 53.36 & 52.69 & 53.11 & 53.37 & 53.51 & 53.51 & 53.37 & 52.89 & 52.67 & 53.29 \\
\hline & 8.0 & 53.52 & 53.49 & 57.20 & 58.34 & 58.52 & 58.52 & 58.34 & 55.60 & 53.47 & 53.44 \\
\hline & 9.0 & 53.95 & 56.75 & 98.09 & 102.43 & 102.65 & 102.65 & 102.43 & 81.06 & 56.74 & 53.92 \\
\hline & 10.0 & 54.13 & 57.04 & 99.53 & 104.12 & 104.37 & 104.37 & 104.12 & 82.07 & 57.03 & 54.08 \\
\hline & 11.0 & 54.35 & 57.45 & 102.16 & 107.01 & 107.29 & 107.29 & 107.01 & 83.77 & 57.43 & 54.27 \\
\hline & 12.0 & 54.54 & 57.75 & 103.91 & 108.90 & 109.20 & 109.20 & 108.90 & 84.90 & 57.73 & 54.46 \\
\hline & 13.0 & 54.73 & 58.02 & 104.45 & 109.53 & 109.85 & 109.85 & 109.53 & 85.30 & 58.00 & 54.66 \\
\hline & 14.0 & 54.89 & 58.32 & 108.02 & 113.31 & 113.65 & 113.65 & 113.31 & 87.48 & 58.31 & 54.82 \\
\hline & 15.0 & 54.72 & 54.05 & 54.98 & 55.55 & 55.88 & 55.88 & 55.55 & 54.52 & 54.02 & 54.62 \\
\hline \multirow{9}{*}{ A-3 } & 15.0 & 54.72 & 54.05 & 54.98 & 55.55 & 55.88 & 55.88 & 55.55 & 54.52 & 54.02 & 54.62 \\
\hline & 16.0 & 55.24 & 58.41 & 104.59 & 109.66 & 110.01 & 110.01 & 109.66 & 85.52 & 58.39 & 55.12 \\
\hline & 17.0 & 55.43 & 58.59 & 104.24 & 109.31 & 109.68 & 109.68 & 109.31 & 85.38 & 58.58 & 55.36 \\
\hline & 18.0 & 55.59 & 58.79 & 104.45 & 109.54 & 109.95 & 109.95 & 109.54 & 85.57 & 58.78 & 55.54 \\
\hline & 19.0 & 55.79 & 59.06 & 105.13 & 110.29 & 110.72 & 110.72 & 110.29 & 86.06 & 59.04 & 55.72 \\
\hline & 20.0 & 55.97 & 59.33 & 106.10 & 111.34 & 111.79 & 111.79 & 111.34 & 86.72 & 59.30 & 55.86 \\
\hline & 21.0 & 56.16 & 59.75 & 108.64 & 114.09 & 114.55 & 114.55 & 114.09 & 88.35 & 59.73 & 56.06 \\
\hline & 22.0 & 56.04 & 56.39 & 61.48 & 63.28 & 63.80 & 63.80 & 63.28 & 59.28 & 56.36 & 55.95 \\
\hline & 22.5 & 56.00 & 55.57 & 56.79 & 57.61 & 58.11 & 58.11 & 57.61 & 56.21 & 55.55 & 55.94 \\
\hline \multirow{9}{*}{ A-4 } & 22.5 & 56.00 & 55.57 & 56.79 & 57.61 & 58.11 & 58.11 & 57.61 & 56.21 & 55.55 & 55.94 \\
\hline & 23.0 & 56.18 & 56.59 & 61.93 & 63.79 & 64.32 & 64.32 & 63.79 & 59.63 & 56.57 & 56.09 \\
\hline & 24.0 & 56.63 & 60.60 & 112.99 & 118.77 & 119.26 & 119.26 & 118.77 & 91.19 & 60.58 & 56.51 \\
\hline & 25.0 & 56.77 & 60.67 & 111.32 & 116.99 & 117.50 & 117.50 & 116.99 & 90.24 & 60.65 & 56.65 \\
\hline & 26.0 & 56.93 & 60.92 & 111.61 & 117.31 & 117.84 & 117.84 & 117.31 & 90.49 & 60.91 & 56.85 \\
\hline & 27.0 & 57.07 & 61.06 & 110.82 & 116.47 & 117.02 & 117.02 & 116.47 & 90.08 & 61.05 & 57.01 \\
\hline & 28.0 & 57.20 & 61.28 & 111.03 & 116.71 & 117.27 & 117.27 & 116.71 & 90.28 & 61.26 & 57.12 \\
\hline & 29.0 & 57.29 & 61.44 & 112.32 & 118.03 & 118.60 & 118.60 & 118.03 & 91.07 & 61.42 & 57.18 \\
\hline & 30.0 & 57.08 & 57.26 & 58.86 & 59.92 & 60.58 & 60.58 & 59.92 & 58.13 & 57.23 & 56.96 \\
\hline \multirow{9}{*}{ A-5 } & 30.0 & 57.08 & 57.26 & 58.86 & 59.92 & 60.58 & 60.58 & 59.92 & 58.13 & 57.23 & 56.96 \\
\hline & 31.0 & 57.53 & 61.82 & 111.98 & 117.68 & 118.25 & 118.25 & 117.68 & 91.03 & 61.80 & 57.44 \\
\hline & 32.0 & 57.65 & 61.86 & 109.43 & 114.97 & 115.57 & 115.57 & 114.97 & 89.56 & 61.84 & 57.55 \\
\hline & 33.0 & 57.74 & 62.00 & 109.37 & 114.88 & 115.49 & 115.49 & 114.88 & 89.58 & 61.98 & 57.63 \\
\hline & 34.0 & 57.85 & 62.11 & 108.08 & 113.50 & 114.13 & 114.13 & 113.50 & 88.87 & 62.09 & 57.73 \\
\hline & 35.0 & 57.93 & 62.18 & 106.66 & 111.97 & 112.61 & 112.61 & 111.97 & 88.07 & 62.16 & 57.84 \\
\hline & 36.0 & 58.01 & 62.27 & 105.45 & 110.65 & 111.30 & 111.30 & 110.65 & 87.40 & 62.26 & 57.95 \\
\hline & 37.0 & 57.86 & 59.44 & 64.42 & 66.41 & 67.17 & 67.17 & 66.41 & 62.31 & 59.42 & 57.78 \\
\hline & 37.5 & 57.80 & 58.78 & 60.50 & 61.67 & 62.42 & 62.42 & 61.67 & 59.75 & 58.75 & 57.69 \\
\hline \multirow{9}{*}{ A-6 } & 37.5 & 57.80 & 58.78 & 60.50 & 61.67 & 62.42 & 62.42 & 61.67 & 59.75 & 58.75 & 57.69 \\
\hline & 38.0 & 57.90 & 59.46 & 64.01 & 65.88 & 66.62 & 66.62 & 65.88 & 62.08 & 59.44 & 57.79 \\
\hline & 39.0 & 58.15 & 62.32 & 101.34 & 106.15 & 106.80 & 106.80 & 106.15 & 85.05 & 62.31 & 58.04 \\
\hline & $\begin{array}{l}40.0 \\
\end{array}$ & 58.18 & 62.20 & 97.75 & 102.26 & 102.93 & 102.93 & 102.26 & 82.92 & 62.18 & 58.09 \\
\hline & 41.0 & 58.21 & 62.11 & 94.62 & 98.87 & 99.54 & 99.54 & 98.87 & 81.08 & 62.10 & 58.12 \\
\hline & 42.0 & 58.23 & 62.06 & 92.04 & 95.99 & 96.67 & 96.67 & 95.99 & 79.52 & 62.04 & 58.12 \\
\hline & 43.0 & 58.24 & 62.10 & 91.74 & 95.61 & 96.28 & 96.28 & 95.61 & 79.36 & 62.08 & 58.14 \\
\hline & 44.0 & 58.20 & 61.91 & 88.37 & 91.83 & 92.45 & 92.45 & 91.83 & 77.27 & 61.90 & 58.15 \\
\hline & 45.0 & 58.04 & 59.86 & 61.51 & 62.66 & 63.40 & 63.40 & 62.66 & 60.82 & 59.85 & 58.01 \\
\hline
\end{tabular}


Table B - 6: Temperature $\left({ }^{\circ} \mathrm{C}\right)$ map of the west side of position A at MOC1 144B (18.0 EFPD).

\begin{tabular}{|c|c|c|c|c|c|c|c|c|c|c|c|}
\hline \multirow{2}{*}{$\begin{array}{c}\text { Plate } \\
\text { Location }\end{array}$} & \multirow{2}{*}{$\begin{array}{c}\text { Axial } \\
\text { Location (in) }\end{array}$} & \multicolumn{10}{|c|}{ Width of Plate (inches) } \\
\hline & & 0.00 & 0.08 & 0.16 & 0.25 & 0.50 & 1.00 & 1.50 & 2.00 & 2.08 & 2.16 \\
\hline \multirow{9}{*}{ A-1 } & 0.0 & 51.67 & 51.76 & 51.76 & 51.76 & 51.76 & 51.76 & 51.76 & 51.76 & 51.76 & 51.67 \\
\hline & 1.0 & 52.61 & 53.92 & 76.52 & 78.98 & 79.05 & 79.05 & 78.98 & 66.84 & 53.92 & 52.58 \\
\hline & 2.0 & 52.80 & 54.30 & 79.96 & 82.70 & 82.80 & 82.80 & 82.70 & 69.04 & 54.29 & 52.73 \\
\hline & 3.0 & 52.98 & 54.53 & 80.56 & 83.41 & 83.52 & 83.52 & 83.41 & 69.49 & 54.53 & 52.89 \\
\hline & 4.0 & 53.20 & 54.95 & 84.03 & 87.14 & 87.29 & 87.29 & 87.14 & 71.74 & 54.94 & 53.12 \\
\hline & 5.0 & 53.40 & 55.35 & 87.06 & 90.42 & 90.59 & 90.59 & 90.42 & 73.74 & 55.34 & 53.30 \\
\hline & 6.0 & 53.63 & 55.91 & 91.54 & 95.25 & 95.44 & 95.44 & 95.25 & 76.69 & 55.90 & 53.51 \\
\hline & 7.0 & 53.56 & 53.41 & 56.69 & 57.73 & 57.93 & 57.93 & 57.73 & 55.27 & 53.40 & 53.42 \\
\hline & 7.5 & 53.55 & 52.84 & 53.35 & 53.65 & 53.83 & 53.83 & 53.65 & 53.09 & 52.83 & 53.40 \\
\hline \multirow{9}{*}{ A-2 } & 7.5 & 53.55 & 52.84 & 53.35 & 53.65 & 53.83 & 53.83 & 53.65 & 53.09 & 52.83 & 53.40 \\
\hline & 8.0 & 53.73 & 53.64 & 57.45 & 58.63 & 58.85 & 58.85 & 58.63 & 55.81 & 53.63 & 53.58 \\
\hline & 9.0 & 54.23 & 56.93 & 98.40 & 102.87 & 103.14 & 103.14 & 102.86 & 81.31 & 56.92 & 54.16 \\
\hline & 10.0 & 54.43 & 57.25 & 99.94 & 104.63 & 104.94 & 104.94 & 104.63 & 82.35 & 57.24 & 54.33 \\
\hline & 11.0 & 54.67 & 57.71 & 102.67 & 107.61 & 107.96 & 107.96 & 107.61 & 84.11 & 57.70 & 54.52 \\
\hline & 12.0 & 54.88 & 58.05 & 104.48 & 109.58 & 109.96 & 109.96 & 109.58 & 85.28 & 58.03 & 54.70 \\
\hline & 13.0 & 55.09 & 58.37 & 105.10 & 110.30 & 110.72 & 110.72 & 110.30 & 85.74 & 58.35 & 54.93 \\
\hline & 14.0 & 55.26 & 58.72 & 108.75 & 114.18 & 114.61 & 114.61 & 114.18 & 87.98 & 58.71 & 55.10 \\
\hline & 15.0 & 55.04 & 54.49 & 55.63 & 56.32 & 56.75 & 56.75 & 56.32 & 55.08 & 54.48 & 54.84 \\
\hline \multirow{9}{*}{ A-3 } & 15.0 & 55.04 & 54.49 & 55.63 & 56.32 & 56.75 & 56.75 & 56.32 & 55.08 & 54.48 & 54.84 \\
\hline & 16.0 & 55.65 & 58.89 & 105.46 & 110.69 & 111.15 & 111.15 & 110.69 & 86.11 & 58.87 & 55.41 \\
\hline & 17.0 & 55.86 & 59.12 & 105.19 & 110.42 & 110.92 & 110.92 & 110.41 & 86.02 & 59.10 & 55.72 \\
\hline & 18.0 & 56.04 & 59.37 & 105.48 & 110.75 & 111.28 & 111.28 & 110.75 & 86.27 & 59.35 & 55.94 \\
\hline & 19.0 & 56.26 & 59.70 & 106.26 & 111.61 & 112.18 & 112.18 & 111.61 & 86.83 & 59.68 & 56.11 \\
\hline & 20.0 & 56.45 & 60.02 & 107.32 & 112.77 & 113.37 & 113.37 & 112.77 & 87.55 & 60.00 & 56.23 \\
\hline & 21.0 & 56.67 & 60.52 & 109.96 & 115.63 & 116.26 & 116.26 & 115.63 & 89.26 & 60.49 & 56.45 \\
\hline & 22.0 & 56.50 & 57.21 & 62.61 & 64.60 & 65.27 & 65.27 & 64.60 & 60.29 & 57.20 & 56.30 \\
\hline & 22.5 & 56.44 & 56.42 & 57.97 & 58.99 & 59.64 & 59.64 & 58.99 & 57.25 & 56.41 & 56.34 \\
\hline \multirow{9}{*}{ A-4 } & 22.5 & 56.44 & 56.42 & 57.97 & 58.99 & 59.64 & 59.64 & 58.99 & 57.25 & 56.41 & 56.34 \\
\hline & 23.0 & 56.65 & 57.46 & 63.11 & 65.17 & 65.84 & 65.84 & 65.17 & 60.68 & 57.44 & 56.45 \\
\hline & 24.0 & 57.18 & 61.52 & 114.52 & 120.54 & 121.21 & 121.21 & 120.54 & 92.25 & 61.49 & 56.92 \\
\hline & 25.0 & 57.33 & 61.64 & 112.94 & 118.86 & 119.56 & 119.56 & 118.86 & 91.37 & 61.62 & 57.09 \\
\hline & 26.0 & 57.51 & 61.97 & 113.33 & 119.30 & 120.03 & 120.03 & 119.29 & 91.70 & 61.94 & 57.35 \\
\hline & 27.0 & 57.66 & 62.17 & 112.64 & 118.57 & 119.33 & 119.33 & 118.57 & 91.36 & 62.15 & 57.53 \\
\hline & 28.0 & 57.81 & 62.45 & 112.95 & 118.92 & 119.70 & 119.70 & 118.91 & 91.63 & 62.43 & 57.64 \\
\hline & 29.0 & 57.90 & 62.68 & 114.34 & 120.35 & 121.14 & 121.14 & 120.35 & 92.49 & 62.65 & 57.66 \\
\hline & 30.0 & 57.63 & 58.58 & 60.58 & 61.92 & 62.78 & 62.78 & 61.92 & 59.68 & 58.55 & 57.38 \\
\hline \multirow{9}{*}{ A-5 } & 30.0 & 57.63 & 58.58 & 60.59 & 61.92 & 62.78 & 62.78 & 61.92 & 59.68 & 58.55 & 57.38 \\
\hline & 31.0 & 58.16 & 63.17 & 114.13 & 120.14 & 120.95 & 120.95 & 120.14 & 92.57 & 63.14 & 57.95 \\
\hline & 32.0 & 58.29 & 63.27 & 111.68 & 117.53 & 118.37 & 118.37 & 117.53 & 91.16 & 63.24 & 58.07 \\
\hline & 33.0 & 58.39 & 63.46 & 111.70 & 117.53 & 118.39 & 118.39 & 117.52 & 91.23 & 63.43 & 58.13 \\
\hline & 34.0 & 58.50 & 63.65 & 110.51 & 116.26 & 117.14 & 117.14 & 116.26 & 90.61 & 63.62 & 58.26 \\
\hline & 35.0 & 58.59 & 63.77 & 109.19 & 114.83 & 115.74 & 115.73 & 114.83 & 89.88 & 63.74 & 58.41 \\
\hline & 36.0 & 58.69 & 63.94 & 108.07 & 113.63 & 114.55 & 114.55 & 113.62 & 89.29 & 63.91 & 58.54 \\
\hline & 37.0 & 58.49 & 61.18 & 66.58 & 68.87 & 69.85 & 69.85 & 68.87 & 64.29 & 61.15 & 58.32 \\
\hline & 37.5 & 58.41 & 60.54 & 62.72 & 64.20 & 65.17 & 65.17 & 64.20 & 61.76 & 60.51 & 58.17 \\
\hline \multirow{9}{*}{ A-6 } & 37.5 & 58.41 & 60.54 & 62.72 & 64.20 & 65.17 & 65.17 & 64.20 & 61.76 & 60.51 & 58.17 \\
\hline & 38.0 & 58.53 & 61.24 & 66.21 & 68.38 & 69.34 & 69.34 & 68.38 & 64.10 & 61.21 & 58.30 \\
\hline & 39.0 & 58.83 & 64.14 & 104.17 & 109.34 & 110.26 & 110.26 & 109.34 & 87.10 & 64.11 & 58.59 \\
\hline & 40.0 & 58.86 & 64.06 & 100.67 & 105.55 & 106.49 & 106.49 & 105.55 & 85.04 & 64.03 & 58.65 \\
\hline & 41.0 & 58.89 & 64.04 & 97.65 & 102.27 & 103.22 & 103.22 & 102.26 & 83.28 & 64.01 & 58.68 \\
\hline & 42.0 & 58.91 & 64.03 & 95.08 & 99.48 & 100.44 & 100.44 & 99.48 & 81.79 & 64.00 & 58.67 \\
\hline & 43.0 & 58.92 & 64.12 & 94.84 & 99.16 & 100.11 & 100.11 & 99.15 & 81.68 & 64.09 & 58.70 \\
\hline & 44.0 & 58.88 & 63.99 & 91.47 & 95.46 & 96.40 & 96.40 & 95.45 & 79.67 & 63.96 & 58.74 \\
\hline & 45.0 & 58.67 & 61.97 & 64.07 & 65.53 & 66.50 & 66.50 & 65.52 & 63.19 & 61.95 & 58.59 \\
\hline
\end{tabular}


Table B - 7: Temperature $\left({ }^{\circ} \mathrm{C}\right)$ map of the east side of position B at MOC1 144B (18.0 EFPD).

\begin{tabular}{|c|c|c|c|c|c|c|c|c|c|c|c|}
\hline \multirow{2}{*}{$\begin{array}{c}\text { Plate } \\
\text { Location }\end{array}$} & \multirow{2}{*}{$\begin{array}{c}\text { Axial } \\
\text { Location (in) }\end{array}$} & \multicolumn{10}{|c|}{ Width of Plate (inches) } \\
\hline & & 0.00 & 0.08 & 0.16 & 0.25 & 0.50 & 1.00 & 1.50 & 2.00 & 2.08 & 2.16 \\
\hline \multirow{9}{*}{ B-1 } & 0.0 & 51.67 & 51.79 & 51.76 & 51.76 & 51.76 & 51.76 & 51.76 & 51.76 & 51.79 & 51.67 \\
\hline & 1.0 & 52.95 & 53.97 & 76.21 & 78.64 & 78.72 & 78.72 & 78.64 & 66.65 & 53.94 & 52.85 \\
\hline & 2.0 & 53.18 & 54.35 & 79.66 & 82.37 & 82.47 & 82.47 & 82.37 & 68.85 & 54.30 & 52.93 \\
\hline & 3.0 & 53.39 & 54.59 & 80.25 & 83.07 & 83.18 & 83.18 & 83.07 & 69.30 & 54.53 & 53.02 \\
\hline & 4.0 & 53.66 & 55.02 & 83.60 & 86.68 & 86.83 & 86.83 & 86.68 & 71.48 & 54.96 & 53.31 \\
\hline & 5.0 & 53.89 & 55.46 & 87.10 & 90.46 & 90.63 & 90.63 & 90.46 & 73.77 & 55.39 & 53.48 \\
\hline & 6.0 & 54.17 & 55.98 & 90.99 & 94.65 & 94.84 & 94.84 & 94.65 & 76.34 & 55.90 & 53.68 \\
\hline & 7.0 & 54.13 & 53.53 & 56.65 & 57.67 & 57.87 & 57.87 & 57.67 & 55.25 & 53.44 & 53.62 \\
\hline & 7.5 & 54.14 & 52.98 & 53.36 & 53.66 & 53.83 & 53.83 & 53.66 & 53.11 & 52.89 & 53.60 \\
\hline \multirow{9}{*}{ B-2 } & 7.5 & 54.14 & 52.98 & 53.36 & 53.66 & 53.83 & 53.83 & 53.66 & 53.11 & 52.89 & 53.60 \\
\hline & 8.0 & 54.34 & 53.75 & 57.24 & 58.38 & 58.59 & 58.59 & 58.38 & 55.68 & 53.65 & 53.77 \\
\hline & 9.0 & 54.86 & 56.88 & 96.23 & 100.37 & 100.64 & 100.64 & 100.37 & 79.91 & 56.85 & 54.77 \\
\hline & 10.0 & 55.09 & 57.16 & 97.19 & 101.51 & 101.82 & 101.82 & 101.51 & 80.60 & 57.10 & 54.84 \\
\hline & 11.0 & 55.37 & 57.59 & 99.43 & 104.09 & 104.43 & 104.43 & 104.09 & 82.11 & 57.49 & 54.87 \\
\hline & 12.0 & 55.60 & 57.98 & 101.80 & 106.67 & 107.04 & 107.04 & 106.66 & 83.63 & 57.87 & 54.92 \\
\hline & 13.0 & 55.84 & 58.31 & 102.41 & 107.38 & 107.79 & 107.79 & 107.38 & 84.09 & 58.20 & 55.20 \\
\hline & 14.0 & 56.04 & 58.63 & 105.44 & 110.60 & 111.03 & 111.03 & 110.60 & 85.95 & 58.52 & 55.35 \\
\hline & 15.0 & 55.84 & 54.70 & 55.64 & 56.31 & 56.73 & 56.73 & 56.31 & 55.11 & 54.57 & 55.12 \\
\hline \multirow{9}{*}{ B-3 } & 15.0 & 55.84 & 54.70 & 55.64 & 56.31 & 56.73 & 56.73 & 56.31 & 55.11 & 54.57 & 55.12 \\
\hline & 16.0 & 56.50 & 59.43 & 109.19 & 114.69 & 115.16 & 115.16 & 114.69 & 88.43 & 59.29 & 55.68 \\
\hline & 17.0 & 56.72 & 59.64 & 108.58 & 114.07 & 114.57 & 114.57 & 114.07 & 88.14 & 59.55 & 56.39 \\
\hline & 18.0 & 56.92 & 59.89 & 108.83 & 114.36 & 114.89 & 114.89 & 114.36 & 88.37 & 59.84 & 56.76 \\
\hline & 19.0 & 57.16 & 60.20 & 109.27 & 114.85 & 115.42 & 115.42 & 114.85 & 88.72 & 60.11 & 56.79 \\
\hline & 20.0 & 57.36 & 60.52 & 110.24 & 115.91 & 116.51 & 116.51 & 115.91 & 89.38 & 60.38 & 56.68 \\
\hline & 21.0 & 57.59 & 61.05 & 113.25 & 119.16 & 119.79 & 119.79 & 119.16 & 91.31 & 60.91 & 56.82 \\
\hline & 22.0 & 57.43 & 57.55 & 63.05 & 65.09 & 65.76 & 65.76 & 65.09 & 60.61 & 57.41 & 56.62 \\
\hline & 22.5 & 57.37 & 56.71 & 58.11 & 59.13 & 59.77 & 59.77 & 59.12 & 57.38 & 56.66 & 57.25 \\
\hline \multirow{9}{*}{ B-4 } & 22.5 & 57.37 & 56.71 & 58.11 & 59.13 & 59.77 & 59.77 & 59.12 & 57.38 & 56.66 & 57.25 \\
\hline & 23.0 & 57.58 & 57.73 & 63.16 & 65.19 & 65.86 & 65.86 & 65.19 & 60.75 & 57.59 & 56.77 \\
\hline & 24.0 & 58.11 & 61.72 & 113.79 & 119.75 & 120.41 & 120.41 & 119.74 & 91.85 & 61.56 & 57.18 \\
\hline & 25.0 & 58.26 & 61.84 & 112.12 & 117.96 & 118.66 & 118.66 & 117.95 & 90.90 & 61.69 & 57.54 \\
\hline & 26.0 & 58.44 & 62.15 & 112.23 & 118.10 & 118.83 & 118.83 & 118.09 & 91.07 & 62.04 & 58.09 \\
\hline & 27.0 & 58.59 & 62.41 & 112.27 & 118.15 & 118.91 & 118.91 & 118.15 & 91.18 & 62.35 & 58.39 \\
\hline & 28.0 & 58.73 & 62.70 & 112.62 & 118.54 & 119.33 & 119.33 & 118.54 & 91.48 & 62.59 & 58.33 \\
\hline & 29.0 & 58.82 & 62.92 & 113.82 & 119.77 & 120.56 & 120.56 & 119.77 & 92.22 & 62.77 & 58.09 \\
\hline & 30.0 & 58.53 & 58.89 & 60.73 & 62.04 & 62.90 & 62.89 & 62.04 & 59.82 & 58.72 & 57.67 \\
\hline \multirow{9}{*}{ B-5 } & 30.0 & 58.53 & 58.89 & 60.73 & 62.04 & 62.90 & 62.89 & 62.04 & 59.82 & 58.72 & 57.67 \\
\hline & 31.0 & 59.05 & 63.18 & 110.89 & 116.64 & 117.45 & 117.44 & 116.63 & 90.63 & 63.05 & 58.32 \\
\hline & 32.0 & 59.16 & 63.26 & 108.22 & 113.78 & 114.62 & 114.62 & 113.77 & 89.10 & 63.11 & 58.34 \\
\hline & 33.0 & 59.25 & 63.45 & 108.22 & 113.76 & 114.62 & 114.62 & 113.76 & 89.17 & 63.29 & 58.34 \\
\hline & 34.0 & 59.34 & 63.64 & 107.16 & 112.62 & 113.50 & 113.50 & 112.61 & 88.61 & 63.49 & 58.60 \\
\hline & 35.0 & 59.40 & 63.75 & 105.58 & 110.91 & 111.81 & 111.81 & 110.91 & 87.73 & 63.64 & 59.00 \\
\hline & 36.0 & 59.47 & 64.01 & 105.64 & 110.97 & 111.89 & 111.89 & 110.97 & 87.87 & 63.94 & 59.18 \\
\hline & 37.0 & 59.26 & 61.42 & 66.47 & 68.69 & 69.65 & 69.65 & 68.68 & 64.26 & 61.31 & 58.94 \\
\hline & 37.5 & 59.17 & 60.83 & 62.85 & 64.30 & 65.26 & 65.26 & 64.29 & 61.90 & 60.67 & 58.38 \\
\hline \multirow{9}{*}{ B-6 } & 37.5 & 59.17 & 60.83 & 62.85 & 64.30 & 65.26 & 65.26 & 64.29 & 61.90 & 60.67 & 58.38 \\
\hline & 38.0 & 59.27 & 61.51 & 66.24 & 68.37 & 69.32 & 69.31 & 68.36 & 64.17 & 61.36 & 58.60 \\
\hline & 39.0 & 59.54 & 64.32 & 103.16 & 108.23 & 109.14 & 109.14 & 108.22 & 86.55 & 64.18 & 58.80 \\
\hline & $\begin{array}{l}40.0 \\
\end{array}$ & 59.54 & 64.23 & 99.49 & 104.25 & 105.18 & 105.18 & 104.24 & 84.38 & 64.09 & 58.81 \\
\hline & 41.0 & 59.54 & 64.21 & 96.61 & 101.12 & 102.06 & 102.06 & 101.11 & 82.71 & 64.08 & 58.87 \\
\hline & 42.0 & 59.53 & 64.20 & 94.12 & 98.41 & 99.36 & 99.36 & 98.40 & 81.26 & 64.06 & 58.77 \\
\hline & 43.0 & 59.51 & 64.29 & 93.91 & 98.12 & 99.07 & 99.07 & 98.11 & 81.18 & 64.16 & 58.91 \\
\hline & 44.0 & 59.43 & 64.18 & 90.91 & 94.82 & 95.76 & 95.75 & 94.81 & 79.39 & 64.09 & 59.15 \\
\hline & 45.0 & 59.21 & 62.23 & 64.23 & 65.66 & 66.62 & 66.62 & 65.65 & 63.35 & 62.18 & 59.07 \\
\hline
\end{tabular}


Table B - 8: Temperature $\left({ }^{\circ} \mathrm{C}\right)$ map of the west side of position B at MOC1 144B (18.0 EFPD).

\begin{tabular}{|c|c|c|c|c|c|c|c|c|c|c|c|}
\hline \multirow{2}{*}{$\begin{array}{c}\text { Plate } \\
\text { Location }\end{array}$} & \multirow{2}{*}{$\begin{array}{c}\text { Axial } \\
\text { Location (in) }\end{array}$} & \multicolumn{10}{|c|}{ Width of Plate (inches) } \\
\hline & & 0.00 & 0.08 & 0.16 & 0.25 & 0.50 & 1.00 & 1.50 & 2.00 & 2.08 & 2.16 \\
\hline \multirow{9}{*}{ B-1 } & 0.0 & 51.67 & 51.78 & 51.76 & 51.76 & 51.77 & 51.77 & 51.76 & 51.76 & 51.78 & 51.67 \\
\hline & 1.0 & 53.26 & 53.95 & 76.20 & 78.63 & 78.70 & 78.70 & 78.63 & 66.64 & 53.93 & 53.14 \\
\hline & 2.0 & 53.51 & 54.32 & 79.63 & 82.33 & 82.42 & 82.42 & 82.33 & 68.83 & 54.28 & 53.08 \\
\hline & 3.0 & 53.76 & 54.54 & 80.20 & 83.00 & 83.10 & 83.10 & 83.00 & 69.26 & 54.50 & 53.05 \\
\hline & 4.0 & 54.05 & 54.94 & 83.51 & 86.57 & 86.69 & 86.69 & 86.56 & 71.41 & 54.90 & 53.37 \\
\hline & 5.0 & 54.31 & 55.36 & 86.97 & 90.30 & 90.44 & 90.44 & 90.30 & 73.67 & 55.31 & 53.49 \\
\hline & 6.0 & 54.61 & 55.86 & 90.80 & 94.43 & 94.59 & 94.59 & 94.43 & 76.20 & 55.80 & 53.70 \\
\hline & 7.0 & 54.63 & 53.38 & 56.46 & 57.44 & 57.60 & 57.60 & 57.44 & 55.09 & 53.31 & 53.73 \\
\hline & 7.5 & 54.67 & 52.81 & 53.15 & 53.40 & 53.54 & 53.54 & 53.40 & 52.93 & 52.74 & 53.73 \\
\hline \multirow{9}{*}{ B-2 } & 7.5 & 54.67 & 52.81 & 53.15 & 53.40 & 53.54 & 53.54 & 53.40 & 52.93 & 52.74 & 53.73 \\
\hline & 8.0 & 54.87 & 53.57 & 57.02 & 58.11 & 58.28 & 58.28 & 58.11 & 55.50 & 53.50 & 53.88 \\
\hline & 9.0 & 55.37 & 56.69 & 95.94 & 99.95 & 100.17 & 100.17 & 99.95 & 79.69 & 56.66 & 55.40 \\
\hline & 10.0 & 55.62 & 56.93 & 96.86 & 101.04 & 101.28 & 101.28 & 101.03 & 80.34 & 56.89 & 55.34 \\
\hline & 11.0 & 55.91 & 57.32 & 98.99 & 103.52 & 103.79 & 103.79 & 103.52 & 81.81 & 57.25 & 55.05 \\
\hline & 12.0 & 56.16 & 57.67 & 101.26 & 106.01 & 106.30 & 106.30 & 106.01 & 83.28 & 57.59 & 54.85 \\
\hline & 13.0 & 56.40 & 57.96 & 101.80 & 106.64 & 106.95 & 106.95 & 106.64 & 83.69 & 57.87 & 55.15 \\
\hline & 14.0 & 56.61 & 58.23 & 104.75 & 109.76 & 110.08 & 110.08 & 109.76 & 85.50 & 58.14 & 55.26 \\
\hline & 15.0 & 56.50 & 54.25 & 55.03 & 55.57 & 55.89 & 55.89 & 55.57 & 54.60 & 54.16 & 55.20 \\
\hline \multirow{9}{*}{ B-3 } & 15.0 & 56.50 & 54.25 & 55.03 & 55.57 & 55.89 & 55.89 & 55.57 & 54.60 & 54.16 & 55.20 \\
\hline & 16.0 & 57.11 & 58.96 & 108.39 & 113.73 & 114.07 & 114.07 & 113.73 & 87.90 & 58.85 & 55.68 \\
\hline & 17.0 & 57.32 & 59.14 & 107.72 & 113.03 & 113.40 & 113.40 & 113.03 & 87.57 & 59.07 & 56.94 \\
\hline & 18.0 & 57.53 & 59.34 & 107.91 & 113.24 & 113.63 & 113.63 & 113.24 & 87.74 & 59.29 & 57.49 \\
\hline & 19.0 & 57.76 & 59.61 & 108.26 & 113.64 & 114.05 & 114.05 & 113.63 & 88.03 & 59.53 & 57.31 \\
\hline & 20.0 & 57.95 & 59.88 & 109.15 & 114.61 & 115.04 & 115.04 & 114.60 & 88.64 & 59.77 & 56.81 \\
\hline & 21.0 & 58.18 & 60.36 & 112.08 & 117.77 & 118.22 & 118.22 & 117.76 & 90.52 & 60.24 & 56.77 \\
\hline & 22.0 & 58.05 & 56.80 & 62.05 & 63.89 & 64.40 & 64.40 & 63.88 & 59.74 & 56.69 & 56.49 \\
\hline & 22.5 & 58.01 & 55.93 & 57.07 & 57.87 & 58.36 & 58.36 & 57.86 & 56.48 & 55.87 & 58.14 \\
\hline \multirow{9}{*}{ B-4 } & 22.5 & 58.01 & 55.93 & 57.07 & 57.87 & 58.36 & 58.36 & 57.86 & 56.48 & 55.87 & 58.14 \\
\hline & 23.0 & 58.20 & 56.94 & 62.12 & 63.94 & 64.46 & 64.45 & 63.93 & 59.83 & 56.83 & 56.62 \\
\hline & 24.0 & 58.67 & 60.90 & 112.44 & 118.14 & 118.61 & 118.61 & 118.13 & 90.92 & 60.77 & 56.94 \\
\hline & 25.0 & 58.80 & 60.98 & 110.69 & 116.26 & 116.76 & 116.75 & 116.25 & 89.91 & 60.85 & 57.58 \\
\hline & 26.0 & 58.95 & 61.22 & 110.71 & 116.29 & 116.81 & 116.81 & 116.28 & 90.01 & 61.12 & 58.60 \\
\hline & 27.0 & 59.09 & 61.43 & 110.66 & 116.25 & 116.78 & 116.78 & 116.24 & 90.06 & 61.35 & 59.04 \\
\hline & 28.0 & 59.21 & 61.66 & 110.93 & 116.54 & 117.09 & 117.08 & 116.53 & 90.29 & 61.56 & 58.74 \\
\hline & 29.0 & 59.27 & 61.83 & 112.03 & 117.66 & 118.21 & 118.21 & 117.65 & 90.97 & 61.69 & 58.04 \\
\hline & 30.0 & 59.03 & 57.72 & 59.19 & 60.22 & 60.86 & 60.86 & 60.21 & 58.45 & 57.58 & 57.47 \\
\hline \multirow{9}{*}{ B-5 } & 30.0 & 59.03 & 57.72 & 59.19 & 60.22 & 60.86 & 60.86 & 60.21 & 58.45 & 57.58 & 57.47 \\
\hline & 31.0 & 59.45 & 61.99 & 108.96 & 114.37 & 114.93 & 114.93 & 114.36 & 89.27 & 61.87 & 58.08 \\
\hline & 32.0 & 59.53 & 62.02 & 106.20 & 111.42 & 112.00 & 112.00 & 111.41 & 87.67 & 61.89 & 57.94 \\
\hline & 33.0 & 59.59 & 62.16 & 106.13 & 111.32 & 111.91 & 111.91 & 111.31 & 87.68 & 62.02 & 57.88 \\
\hline & 34.0 & 59.64 & 62.29 & 104.97 & 110.06 & 110.67 & 110.67 & 110.05 & 87.05 & 62.15 & 58.39 \\
\hline & 35.0 & 59.67 & 62.34 & 103.29 & 108.25 & 108.87 & 108.86 & 108.23 & 86.10 & 62.22 & 59.20 \\
\hline & 36.0 & 59.70 & 62.54 & 103.26 & 108.21 & 108.83 & 108.83 & 108.19 & 86.16 & 62.44 & 59.40 \\
\hline & 37.0 & 59.49 & 59.88 & 64.52 & 66.41 & 67.13 & 67.13 & 66.39 & 62.49 & 59.77 & 59.21 \\
\hline & 37.5 & 59.40 & 59.26 & 60.85 & 61.97 & 62.67 & 62.67 & 61.95 & 60.09 & 59.12 & 57.97 \\
\hline \multirow{9}{*}{ B-6 } & 37.5 & 59.40 & 59.26 & 60.85 & 61.97 & 62.67 & 62.67 & 61.95 & 60.09 & 59.12 & 57.97 \\
\hline & 38.0 & 59.47 & 59.93 & 64.25 & 66.05 & 66.76 & 66.75 & 66.03 & 62.35 & 59.79 & 58.33 \\
\hline & 39.0 & 59.65 & 62.72 & 100.60 & 105.27 & 105.89 & 105.88 & 105.25 & 84.69 & 62.57 & 58.31 \\
\hline & 40.0 & 59.62 & 62.58 & 96.84 & 101.19 & 101.83 & 101.82 & 101.18 & 82.46 & 62.44 & 58.18 \\
\hline & 41.0 & 59.57 & 62.51 & 93.86 & 97.96 & 98.60 & 98.59 & 97.94 & 80.71 & 62.37 & 58.33 \\
\hline & 42.0 & 59.52 & 62.46 & 91.36 & 95.17 & 95.81 & 95.80 & 95.15 & 79.20 & 62.31 & 58.11 \\
\hline & 43.0 & 59.46 & 62.50 & 91.10 & 94.82 & 95.46 & 95.45 & 94.80 & 79.06 & 62.36 & 58.46 \\
\hline & 44.0 & 59.34 & 62.35 & 88.09 & 91.45 & 92.04 & 92.04 & 91.43 & 77.21 & 62.24 & 58.94 \\
\hline & 45.0 & 59.12 & 60.35 & 61.91 & 63.00 & 63.70 & 63.70 & 62.98 & 61.21 & 60.27 & 58.98 \\
\hline
\end{tabular}


Table B - 9: Temperature $\left({ }^{\circ} \mathrm{C}\right)$ map of the east side of position A at MOC2 144B (38.0 EFPD).

\begin{tabular}{|c|c|c|c|c|c|c|c|c|c|c|c|}
\hline \multirow{2}{*}{$\begin{array}{c}\text { Plate } \\
\text { Location }\end{array}$} & \multirow{2}{*}{$\begin{array}{c}\text { Axial } \\
\text { Location (in) }\end{array}$} & \multicolumn{10}{|c|}{ Width of Plate (inches) } \\
\hline & & 0.00 & 0.08 & 0.16 & 0.25 & 0.50 & 1.00 & 1.50 & 2.00 & 2.08 & 2.16 \\
\hline \multirow{9}{*}{ A-1 } & 0.0 & 51.67 & 51.77 & 51.76 & 51.76 & 51.76 & 51.76 & 51.76 & 51.76 & 51.77 & 51.67 \\
\hline & 1.0 & 52.49 & 53.88 & 75.94 & 78.35 & 78.42 & 78.42 & 78.15 & 66.47 & 53.87 & 52.47 \\
\hline & 2.0 & 52.65 & 54.23 & 79.21 & 81.88 & 81.97 & 81.97 & 81.66 & 68.56 & 54.22 & 52.62 \\
\hline & 3.0 & 52.81 & 54.43 & 79.76 & 82.53 & 82.63 & 82.63 & 82.28 & 68.97 & 54.43 & 52.77 \\
\hline & 4.0 & 53.01 & 54.81 & 83.04 & 86.06 & 86.19 & 86.19 & 85.79 & 71.09 & 54.81 & 52.97 \\
\hline & 5.0 & 53.18 & 55.16 & 85.84 & 89.08 & 89.22 & 89.22 & 88.80 & 72.92 & 55.15 & 53.14 \\
\hline & 6.0 & 53.39 & 55.65 & 89.78 & 93.33 & 93.49 & 93.49 & 93.03 & 75.52 & 55.64 & 53.33 \\
\hline & 7.0 & 53.35 & 53.22 & 56.29 & 57.25 & 57.41 & 57.41 & 57.00 & 54.96 & 53.21 & 53.28 \\
\hline & 7.5 & 53.36 & 52.67 & 53.07 & 53.32 & 53.45 & 53.45 & 53.21 & 52.85 & 52.65 & 53.28 \\
\hline \multirow{9}{*}{ A-2 } & 7.5 & 53.36 & 52.67 & 53.07 & 53.32 & 53.45 & 53.45 & 53.21 & 52.85 & 52.65 & 53.28 \\
\hline & 8.0 & 53.51 & 53.43 & 56.98 & 58.07 & 58.24 & 58.24 & 57.79 & 55.44 & 53.41 & 53.43 \\
\hline & 9.0 & 53.92 & 56.55 & 96.22 & 100.28 & 100.50 & 100.50 & 99.91 & 79.84 & 56.55 & 53.89 \\
\hline & 10.0 & 54.10 & 56.84 & 97.68 & 101.99 & 102.23 & 102.23 & 101.58 & 80.84 & 56.83 & 54.05 \\
\hline & 11.0 & 54.31 & 57.22 & 99.93 & 104.59 & 104.86 & 104.86 & 104.15 & 82.37 & 57.20 & 54.24 \\
\hline & 12.0 & 54.50 & 57.49 & 101.44 & 106.23 & 106.52 & 106.52 & 105.77 & 83.35 & 57.47 & 54.42 \\
\hline & 13.0 & 54.68 & 57.74 & 101.91 & 106.78 & 107.10 & 107.10 & 106.30 & 83.72 & 57.73 & 54.61 \\
\hline & 14.0 & 54.84 & 58.00 & 104.90 & 109.95 & 110.27 & 110.27 & 109.48 & 85.55 & 57.98 & 54.77 \\
\hline & 15.0 & 54.69 & 53.97 & 54.84 & 55.38 & 55.70 & 55.70 & 55.14 & 54.40 & 53.94 & 54.59 \\
\hline \multirow{9}{*}{ A-3 } & 15.0 & 54.69 & 53.97 & 54.84 & 55.38 & 55.70 & 55.70 & 55.14 & 54.40 & 53.94 & 54.59 \\
\hline & 16.0 & 55.18 & 58.03 & 101.06 & 105.84 & 106.17 & 106.17 & 105.35 & 83.32 & 58.01 & 55.06 \\
\hline & 17.0 & 55.36 & 58.22 & 100.99 & 105.78 & 106.14 & 106.14 & 105.28 & 83.34 & 58.21 & 55.29 \\
\hline & 18.0 & 55.52 & 58.41 & 101.19 & 106.01 & 106.40 & 106.40 & 105.49 & 83.53 & 58.40 & 55.47 \\
\hline & 19.0 & 55.71 & 58.66 & 101.68 & 106.55 & 106.96 & 106.96 & 106.02 & 83.90 & 58.64 & 55.64 \\
\hline & 20.0 & 55.88 & 58.93 & 102.83 & 107.80 & 108.22 & 108.22 & 107.25 & 84.66 & 58.90 & 55.78 \\
\hline & 21.0 & 56.07 & 59.27 & 104.56 & 109.67 & 110.11 & 110.11 & 109.11 & 85.80 & 59.25 & 55.96 \\
\hline & 22.0 & 55.96 & 56.15 & 60.85 & 62.54 & 63.03 & 63.03 & 62.01 & 58.82 & 56.13 & 55.87 \\
\hline & 22.5 & 55.92 & 55.40 & 56.52 & 57.29 & 57.76 & 57.76 & 56.94 & 55.98 & 55.38 & 55.87 \\
\hline \multirow{9}{*}{ A-4 } & 22.5 & 55.92 & 55.40 & 56.52 & 57.29 & 57.76 & 57.76 & 56.94 & 55.98 & 55.38 & 55.87 \\
\hline & 23.0 & 56.09 & 56.36 & 61.34 & 63.09 & 63.59 & 63.59 & 62.55 & 59.19 & 56.33 & 56.00 \\
\hline & 24.0 & 56.52 & 60.13 & 109.34 & 114.81 & 115.28 & 115.28 & 114.22 & 88.89 & 60.11 & 56.40 \\
\hline & 25.0 & 56.65 & 60.21 & 108.01 & 113.40 & 113.89 & 113.89 & 112.79 & 88.15 & 60.19 & 56.54 \\
\hline & 26.0 & 56.81 & 60.45 & 108.26 & 113.68 & 114.19 & 114.19 & 113.05 & 88.37 & 60.44 & 56.73 \\
\hline & 27.0 & 56.93 & 60.57 & 107.37 & 112.74 & 113.26 & 113.26 & 112.10 & 87.90 & 60.56 & 56.88 \\
\hline & 28.0 & 57.06 & 60.77 & 107.55 & 112.93 & 113.47 & 113.47 & 112.29 & 88.07 & 60.75 & 56.98 \\
\hline & 29.0 & 57.14 & 60.90 & 108.52 & 113.92 & 114.46 & 114.46 & 113.29 & 88.67 & 60.88 & 57.03 \\
\hline & 30.0 & 56.95 & 56.98 & 58.46 & 59.45 & 60.07 & 60.07 & 58.99 & 57.78 & 56.96 & 56.83 \\
\hline \multirow{9}{*}{ A-5 } & 30.0 & 56.95 & 56.98 & 58.46 & 59.45 & 60.07 & 60.07 & 58.99 & 57.78 & 56.96 & 56.83 \\
\hline & 31.0 & 57.37 & 61.29 & 108.60 & 114.02 & 114.57 & 114.57 & 113.37 & 88.88 & 61.27 & 57.28 \\
\hline & 32.0 & 57.48 & 61.35 & 106.45 & 111.73 & 112.30 & 112.30 & 111.06 & 87.65 & 61.33 & 57.39 \\
\hline & 33.0 & 57.57 & 61.48 & 106.42 & 111.68 & 112.26 & 112.26 & 111.01 & 87.68 & 61.46 & 57.46 \\
\hline & 34.0 & 57.67 & 61.60 & 105.37 & 110.55 & 111.15 & 111.15 & 109.87 & 87.11 & 61.58 & 57.56 \\
\hline & 35.0 & 57.74 & 61.67 & 104.06 & 109.14 & 109.75 & 109.75 & 108.46 & 86.37 & 61.65 & 57.66 \\
\hline & 36.0 & 57.82 & 61.75 & 102.81 & 107.78 & 108.41 & 108.41 & 107.10 & 85.68 & 61.74 & 57.76 \\
\hline & 37.0 & 57.67 & 59.04 & 63.75 & 65.63 & 66.34 & 66.34 & 64.97 & 61.75 & 59.02 & 57.59 \\
\hline & 37.5 & 57.61 & 58.41 & 60.01 & 61.11 & 61.81 & 61.81 & 60.59 & 59.31 & 58.38 & 57.50 \\
\hline \multirow{9}{*}{ A-6 } & 37.5 & 57.61 & 58.41 & 60.01 & 61.11 & 61.81 & 61.81 & 60.59 & 59.31 & 58.38 & 57.50 \\
\hline & 38.0 & 57.70 & 59.06 & 63.37 & 65.15 & 65.84 & 65.84 & 64.51 & 61.54 & 59.04 & 57.60 \\
\hline & 39.0 & 57.94 & 61.81 & 99.14 & 103.76 & 104.38 & 104.38 & 103.10 & 83.58 & 61.80 & 57.84 \\
\hline & $\begin{array}{l}40.0 \\
\end{array}$ & 57.97 & 61.73 & 96.14 & 100.51 & 101.14 & 101.14 & 99.85 & 81.81 & 61.71 & 57.88 \\
\hline & 41.0 & 57.99 & 61.65 & 93.31 & 97.38 & 98.02 & 98.02 & 96.73 & 80.11 & 61.64 & 57.91 \\
\hline & 42.0 & 58.01 & 61.59 & 90.74 & 94.49 & 95.13 & 95.13 & 93.85 & 78.54 & 61.57 & 57.91 \\
\hline & 43.0 & 58.01 & 61.62 & 90.45 & 94.13 & 94.76 & 94.76 & 93.50 & 78.39 & 61.61 & 57.92 \\
\hline & 44.0 & 57.98 & 61.45 & 87.33 & 90.70 & 91.28 & 91.28 & 90.13 & 76.45 & 61.44 & 57.93 \\
\hline & 45.0 & 57.81 & 59.43 & 60.99 & 62.07 & 62.77 & 62.77 & 61.55 & 60.34 & 59.43 & 57.78 \\
\hline
\end{tabular}


Table B - 10: Temperature $\left({ }^{\circ} \mathrm{C}\right)$ map of the west side of position A at MOC2 144B (38.0 EFPD).

\begin{tabular}{|c|c|c|c|c|c|c|c|c|c|c|c|}
\hline \multirow{2}{*}{$\begin{array}{c}\text { Plate } \\
\text { Location }\end{array}$} & \multirow{2}{*}{$\begin{array}{c}\text { Axial } \\
\text { Location (in) }\end{array}$} & \multicolumn{10}{|c|}{ Width of Plate (inches) } \\
\hline & & 0.00 & 0.08 & 0.16 & 0.25 & 0.50 & 1.00 & 1.50 & 2.00 & 2.08 & 2.16 \\
\hline \multirow{9}{*}{ A-1 } & 0.0 & 51.67 & 51.76 & 51.76 & 51.76 & 51.76 & 51.76 & 51.76 & 51.76 & 51.76 & 51.67 \\
\hline & 1.0 & 52.61 & 53.87 & 75.95 & 78.37 & 78.44 & 78.44 & 78.37 & 66.48 & 53.87 & 52.57 \\
\hline & 2.0 & 52.79 & 54.23 & 79.24 & 81.92 & 82.01 & 82.01 & 81.92 & 68.58 & 54.23 & 52.72 \\
\hline & 3.0 & 52.97 & 54.46 & 79.82 & 82.60 & 82.71 & 82.71 & 82.60 & 69.02 & 54.45 & 52.88 \\
\hline & 4.0 & 53.19 & 54.86 & 83.14 & 86.19 & 86.33 & 86.33 & 86.19 & 71.17 & 54.85 & 53.11 \\
\hline & 5.0 & 53.38 & 55.23 & 85.98 & 89.26 & 89.42 & 89.42 & 89.26 & 73.03 & 55.23 & 53.29 \\
\hline & 6.0 & 53.61 & 55.75 & 89.97 & 93.57 & 93.75 & 93.75 & 93.57 & 75.67 & 55.74 & 53.49 \\
\hline & 7.0 & 53.55 & 53.35 & 56.49 & 57.49 & 57.68 & 57.68 & 57.49 & 55.13 & 53.34 & 53.41 \\
\hline & 7.5 & 53.54 & 52.81 & 53.29 & 53.58 & 53.75 & 53.75 & 53.58 & 53.04 & 52.80 & 53.39 \\
\hline \multirow{9}{*}{ A-2 } & 7.5 & 53.54 & 52.81 & 53.29 & 53.58 & 53.75 & 53.75 & 53.58 & 53.04 & 52.80 & 53.39 \\
\hline & 8.0 & 53.72 & 53.58 & 57.21 & 58.35 & 58.56 & 58.56 & 58.35 & 55.64 & 53.57 & 53.56 \\
\hline & 9.0 & 54.19 & 56.73 & 96.53 & 100.70 & 100.97 & 100.97 & 100.70 & 80.07 & 56.72 & 54.13 \\
\hline & 10.0 & 54.39 & 57.04 & 98.03 & 102.48 & 102.78 & 102.78 & 102.48 & 81.12 & 57.03 & 54.29 \\
\hline & 11.0 & 54.63 & 57.47 & 100.41 & 105.16 & 105.50 & 105.50 & 105.16 & 82.70 & 57.45 & 54.48 \\
\hline & 12.0 & 54.83 & 57.78 & 101.99 & 106.88 & 107.25 & 107.25 & 106.88 & 83.72 & 57.76 & 54.66 \\
\hline & 13.0 & 55.03 & 58.07 & 102.54 & 107.52 & 107.92 & 107.92 & 107.52 & 84.14 & 58.06 & 54.88 \\
\hline & 14.0 & 55.20 & 58.38 & 105.61 & 110.78 & 111.20 & 111.20 & 110.78 & 86.02 & 58.36 & 55.04 \\
\hline & 15.0 & 55.00 & 54.39 & 55.45 & 56.11 & 56.52 & 56.52 & 56.11 & 54.94 & 54.38 & 54.80 \\
\hline \multirow{9}{*}{ A-3 } & 15.0 & 55.00 & 54.39 & 55.45 & 56.11 & 56.52 & 56.52 & 56.11 & 54.94 & 54.38 & 54.80 \\
\hline & 16.0 & 55.58 & 58.49 & 101.89 & 106.82 & 107.26 & 107.26 & 106.82 & 83.88 & 58.47 & 55.34 \\
\hline & 17.0 & 55.78 & 58.72 & 101.89 & 106.84 & 107.32 & 107.32 & 106.83 & 83.95 & 58.70 & 55.64 \\
\hline & 18.0 & 55.96 & 58.96 & 102.17 & 107.16 & 107.67 & 107.67 & 107.16 & 84.19 & 58.94 & 55.86 \\
\hline & 19.0 & 56.17 & 59.26 & 102.76 & 107.81 & 108.35 & 108.35 & 107.81 & 84.63 & 59.24 & 56.02 \\
\hline & 20.0 & 56.35 & 59.58 & 103.99 & 109.15 & 109.72 & 109.72 & 109.15 & 85.45 & 59.56 & 56.14 \\
\hline & 21.0 & 56.56 & 59.99 & 105.81 & 111.13 & 111.73 & 111.73 & 111.13 & 86.66 & 59.96 & 56.34 \\
\hline & 22.0 & 56.40 & 56.93 & 61.91 & 63.77 & 64.40 & 64.40 & 63.77 & 59.76 & 56.91 & 56.21 \\
\hline & 22.5 & 56.35 & 56.20 & 57.62 & 58.58 & 59.19 & 59.19 & 58.58 & 56.95 & 56.18 & 56.25 \\
\hline \multirow{9}{*}{ A-4 } & 22.5 & 56.35 & 56.20 & 57.62 & 58.58 & 59.19 & 59.19 & 58.58 & 56.95 & 56.18 & 56.25 \\
\hline & 23.0 & 56.55 & 57.17 & 62.44 & 64.38 & 65.02 & 65.02 & 64.38 & 60.18 & 57.16 & 56.35 \\
\hline & 24.0 & 57.04 & 60.99 & 110.78 & 116.49 & 117.12 & 117.12 & 116.49 & 89.90 & 60.96 & 56.79 \\
\hline & 25.0 & 57.19 & 61.13 & 109.54 & 115.17 & 115.83 & 115.83 & 115.17 & 89.21 & 61.10 & 56.96 \\
\hline & 26.0 & 57.36 & 61.43 & 109.88 & 115.55 & 116.25 & 116.25 & 115.55 & 89.51 & 61.40 & 57.20 \\
\hline & 27.0 & 57.50 & 61.61 & 109.09 & 114.71 & 115.44 & 115.44 & 114.71 & 89.10 & 61.58 & 57.38 \\
\hline & 28.0 & 57.64 & 61.86 & 109.36 & 115.01 & 115.76 & 115.76 & 115.01 & 89.34 & 61.84 & 57.47 \\
\hline & 29.0 & 57.73 & 62.06 & 110.42 & 116.10 & 116.85 & 116.85 & 116.10 & 90.01 & 62.03 & 57.50 \\
\hline & 30.0 & 57.47 & 58.21 & 60.07 & 61.31 & 62.12 & 62.12 & 61.31 & 59.22 & 58.19 & 57.23 \\
\hline \multirow{9}{*}{ A-5 } & 30.0 & 57.47 & 58.21 & 60.07 & 61.31 & 62.12 & 62.12 & 61.31 & 59.22 & 58.19 & 57.23 \\
\hline & 31.0 & 57.97 & 62.55 & 110.63 & 116.34 & 117.10 & 117.10 & 116.34 & 90.31 & 62.53 & 57.77 \\
\hline & 32.0 & 58.10 & 62.66 & 108.56 & 114.13 & 114.93 & 114.93 & 114.13 & 89.14 & 62.63 & 57.88 \\
\hline & 33.0 & 58.19 & 62.85 & 108.60 & 114.16 & 114.98 & 114.97 & 114.16 & 89.23 & 62.82 & 57.94 \\
\hline & 34.0 & 58.30 & 63.04 & 107.65 & 113.14 & 113.97 & 113.97 & 113.14 & 88.74 & 63.00 & 58.06 \\
\hline & 35.0 & 58.38 & 63.15 & 106.43 & 111.82 & 112.68 & 112.67 & 111.82 & 88.07 & 63.13 & 58.20 \\
\hline & 36.0 & 58.46 & 63.30 & 105.28 & 110.57 & 111.44 & 111.44 & 110.57 & 87.45 & 63.28 & 58.32 \\
\hline & 37.0 & 58.27 & 60.66 & 65.76 & 67.92 & 68.84 & 68.83 & 67.92 & 63.59 & 60.63 & 58.11 \\
\hline & 37.5 & 58.20 & 60.05 & 62.07 & 63.46 & 64.37 & 64.37 & 63.46 & 61.18 & 60.02 & 57.96 \\
\hline \multirow{9}{*}{ A-6 } & 37.5 & 58.20 & 60.05 & 62.07 & 63.46 & 64.37 & 64.37 & 63.46 & 61.18 & 60.02 & 57.96 \\
\hline & 38.0 & 58.31 & 60.72 & 65.42 & 67.48 & 68.38 & 68.38 & 67.47 & 63.42 & 60.69 & 58.08 \\
\hline & 39.0 & 58.59 & 63.50 & 101.80 & 106.75 & 107.62 & 107.62 & 106.75 & 85.50 & 63.47 & 58.36 \\
\hline & $\begin{array}{l}40.0 \\
\end{array}$ & 58.62 & 63.46 & 98.88 & 103.59 & 104.47 & 104.47 & 103.58 & 83.79 & 63.43 & 58.41 \\
\hline & 41.0 & 58.64 & 63.44 & 96.10 & 100.56 & 101.45 & 101.45 & 100.55 & 82.17 & 63.41 & 58.44 \\
\hline & 42.0 & 58.65 & 63.43 & 93.52 & 97.76 & 98.66 & 98.66 & 97.75 & 80.67 & 63.40 & 58.43 \\
\hline & 43.0 & 58.66 & 63.51 & 93.29 & 97.45 & 98.35 & 98.35 & 97.45 & 80.56 & 63.48 & 58.45 \\
\hline & 44.0 & 58.62 & 63.39 & 90.18 & 94.04 & 94.93 & 94.93 & 94.04 & 78.70 & 63.36 & 58.49 \\
\hline & 45.0 & 58.41 & 61.41 & 63.38 & 64.75 & 65.66 & 65.66 & 64.74 & 62.54 & 61.38 & 58.33 \\
\hline
\end{tabular}


Table B - 11: Temperature $\left({ }^{\circ} \mathrm{C}\right)$ map of the east side of position B at MOC2 144B (38.0 EFPD).

\begin{tabular}{|c|c|c|c|c|c|c|c|c|c|c|c|}
\hline \multirow{2}{*}{$\begin{array}{c}\text { Plate } \\
\text { Location }\end{array}$} & \multirow{2}{*}{$\begin{array}{c}\text { Axial } \\
\text { Location (in) }\end{array}$} & \multicolumn{10}{|c|}{ Width of Plate (inches) } \\
\hline & & 0.00 & 0.08 & 0.16 & 0.25 & 0.50 & 1.00 & 1.50 & 2.00 & 2.08 & 2.16 \\
\hline \multirow{9}{*}{ B-1 } & 0.0 & 51.67 & 51.79 & 51.76 & 51.76 & 51.76 & 51.76 & 51.76 & 51.76 & 51.79 & 51.67 \\
\hline & 1.0 & 52.94 & 53.91 & 75.48 & 77.85 & 77.92 & 77.92 & 77.65 & 66.19 & 53.88 & 52.84 \\
\hline & 2.0 & 53.17 & 54.27 & 78.74 & 81.37 & 81.47 & 81.47 & 81.15 & 68.27 & 54.22 & 52.92 \\
\hline & 3.0 & 53.38 & 54.51 & 79.31 & 82.05 & 82.16 & 82.16 & 81.80 & 68.70 & 54.44 & 53.01 \\
\hline & 4.0 & 53.64 & 54.92 & 82.65 & 85.66 & 85.80 & 85.80 & 85.38 & 70.87 & 54.86 & 53.30 \\
\hline & 5.0 & 53.88 & 55.34 & 85.95 & 89.22 & 89.38 & 89.38 & 88.92 & 73.02 & 55.28 & 53.47 \\
\hline & 6.0 & 54.15 & 55.82 & 89.43 & 92.98 & 93.17 & 93.17 & 92.65 & 75.33 & 55.74 & 53.66 \\
\hline & 7.0 & 54.12 & 53.48 & 56.45 & 57.43 & 57.62 & 57.62 & 57.17 & 55.11 & 53.39 & 53.60 \\
\hline & 7.5 & 54.13 & 52.95 & 53.31 & 53.59 & 53.76 & 53.76 & 53.46 & 53.06 & 52.86 & 53.59 \\
\hline \multirow{9}{*}{ B-2 } & 7.5 & 54.13 & 52.95 & 53.31 & 53.59 & 53.76 & 53.76 & 53.46 & 53.06 & 52.86 & 53.59 \\
\hline & 8.0 & 54.32 & 53.68 & 56.99 & 58.07 & 58.27 & 58.27 & 57.78 & 55.51 & 53.59 & 53.76 \\
\hline & 9.0 & 54.83 & 56.66 & 94.17 & 98.08 & 98.31 & 98.31 & 97.72 & 78.55 & 56.63 & 54.73 \\
\hline & 10.0 & 55.06 & 56.94 & 95.20 & 99.23 & 99.52 & 99.52 & 98.82 & 79.28 & 56.87 & 54.81 \\
\hline & 11.0 & 55.32 & 57.34 & 97.17 & 101.53 & 101.87 & 101.87 & 101.08 & 80.65 & 57.24 & 54.83 \\
\hline & 12.0 & 55.55 & 57.71 & 99.26 & 103.91 & 104.27 & 104.27 & 103.42 & 82.04 & 57.59 & 54.87 \\
\hline & 13.0 & 55.78 & 58.01 & 99.80 & 104.55 & 104.94 & 104.94 & 104.03 & 82.46 & 57.90 & 55.15 \\
\hline & 14.0 & 55.98 & 58.28 & 102.19 & 107.08 & 107.49 & 107.49 & 106.56 & 83.93 & 58.16 & 55.29 \\
\hline & 15.0 & 55.80 & 54.60 & 55.46 & 56.10 & 56.50 & 56.50 & 55.80 & 54.96 & 54.47 & 55.08 \\
\hline \multirow{9}{*}{ B-3 } & 15.0 & 55.80 & 54.60 & 55.46 & 56.10 & 56.50 & 56.50 & 55.80 & 54.96 & 54.47 & 55.08 \\
\hline & 16.0 & 56.42 & 59.00 & 105.39 & 110.59 & 111.03 & 111.03 & 110.02 & 86.06 & 58.87 & 55.61 \\
\hline & 17.0 & 56.64 & 59.22 & 105.02 & 110.21 & 110.69 & 110.69 & 109.61 & 85.91 & 59.13 & 56.31 \\
\hline & 18.0 & 56.83 & 59.46 & 105.28 & 110.51 & 111.02 & 111.02 & 109.89 & 86.14 & 59.41 & 56.67 \\
\hline & 19.0 & 57.06 & 59.76 & 105.85 & 111.14 & 111.68 & 111.68 & 110.50 & 86.57 & 59.67 & 56.70 \\
\hline & 20.0 & 57.25 & 60.06 & 106.64 & 112.00 & 112.57 & 112.57 & 111.34 & 87.12 & 59.92 & 56.59 \\
\hline & 21.0 & 57.47 & 60.51 & 108.89 & 114.44 & 115.04 & 115.04 & 113.76 & 88.58 & 60.37 & 56.71 \\
\hline & 22.0 & 57.32 & 57.26 & 62.33 & 64.24 & 64.86 & 64.86 & 63.60 & 60.07 & 57.12 & 56.53 \\
\hline & 22.5 & 57.27 & 56.48 & 57.76 & 58.71 & 59.31 & 59.31 & 58.26 & 57.08 & 56.43 & 57.16 \\
\hline \multirow{9}{*}{ B-4 } & 22.5 & 57.27 & 56.48 & 57.76 & 58.71 & 59.31 & 59.31 & 58.26 & 57.08 & 56.43 & 57.16 \\
\hline & 23.0 & 57.47 & 57.44 & 62.45 & 64.36 & 64.99 & 64.99 & 63.73 & 60.22 & 57.29 & 56.67 \\
\hline & 24.0 & 57.97 & 61.16 & 109.69 & 115.29 & 115.93 & 115.92 & 114.58 & 89.26 & 61.00 & 57.06 \\
\hline & 25.0 & 58.11 & 61.30 & 108.45 & 113.98 & 114.64 & 114.64 & 113.25 & 88.58 & 61.15 & 57.41 \\
\hline & 26.0 & 58.29 & 61.61 & 108.83 & 114.40 & 115.09 & 115.09 & 113.65 & 88.91 & 61.50 & 57.94 \\
\hline & 27.0 & 58.43 & 61.83 & 108.55 & 114.11 & 114.83 & 114.83 & 113.34 & 88.82 & 61.78 & 58.23 \\
\hline & 28.0 & 58.56 & 62.09 & 108.84 & 114.43 & 115.17 & 115.17 & 113.65 & 89.07 & 61.99 & 58.17 \\
\hline & 29.0 & 58.64 & 62.28 & 109.70 & 115.30 & 116.05 & 116.05 & 114.53 & 89.61 & 62.13 & 57.93 \\
\hline & 30.0 & 58.37 & 58.52 & 60.20 & 61.43 & 62.22 & 62.22 & 60.83 & 59.36 & 58.36 & 57.53 \\
\hline \multirow{9}{*}{ B-5 } & 30.0 & 58.37 & 58.52 & 60.20 & 61.43 & 62.22 & 62.22 & 60.83 & 59.36 & 58.36 & 57.53 \\
\hline & 31.0 & 58.85 & 62.54 & 107.20 & 112.62 & 113.39 & 113.39 & 111.83 & 88.27 & 62.41 & 58.14 \\
\hline & 32.0 & 58.96 & 62.64 & 105.06 & 110.33 & 111.13 & 111.13 & 109.53 & 87.05 & 62.50 & 58.16 \\
\hline & 33.0 & 59.04 & 62.83 & 105.09 & 110.35 & 111.17 & 111.16 & 109.54 & 87.13 & 62.67 & 58.16 \\
\hline & 34.0 & 59.12 & 63.01 & 104.15 & 109.34 & 110.17 & 110.17 & 108.51 & 86.65 & 62.86 & 58.41 \\
\hline & 35.0 & 59.18 & 63.11 & 102.71 & 107.79 & 108.64 & 108.64 & 106.95 & 85.85 & 63.01 & 58.79 \\
\hline & 36.0 & 59.24 & 63.36 & 102.75 & 107.82 & 108.68 & 108.68 & 106.97 & 85.96 & 63.29 & 58.96 \\
\hline & 37.0 & 59.03 & 60.90 & 65.63 & 67.72 & 68.62 & 68.62 & 66.92 & 63.56 & 60.79 & 58.72 \\
\hline & 37.5 & 58.95 & 60.33 & 62.20 & 63.55 & 64.45 & 64.44 & 62.89 & 61.31 & 60.18 & 58.18 \\
\hline \multirow{9}{*}{ B-6 } & 37.5 & 58.95 & 60.33 & 62.20 & 63.55 & 64.45 & 64.44 & 62.89 & 61.31 & 60.18 & 58.18 \\
\hline & 38.0 & 59.04 & 60.98 & 65.44 & 67.44 & 68.33 & 68.33 & 66.66 & 63.48 & 60.84 & 58.39 \\
\hline & 39.0 & 59.29 & 63.67 & 100.66 & 105.50 & 106.36 & 106.36 & 104.67 & 84.86 & 63.53 & 58.57 \\
\hline & 40.0 & 59.29 & 63.62 & 97.72 & 102.31 & 103.19 & 103.19 & 101.49 & 83.14 & 63.49 & 58.58 \\
\hline & 41.0 & 59.28 & 63.59 & 94.87 & 99.20 & 100.09 & 100.09 & 98.38 & 81.48 & 63.47 & 58.64 \\
\hline & 42.0 & 59.26 & 63.58 & 92.38 & 96.49 & 97.39 & 97.38 & 95.67 & 80.03 & 63.44 & 58.53 \\
\hline & 43.0 & 59.24 & 63.66 & 92.18 & 96.22 & 97.12 & 97.11 & 95.42 & 79.95 & 63.53 & 58.67 \\
\hline & 44.0 & 59.16 & 63.56 & 89.48 & 93.26 & 94.14 & 94.13 & 92.48 & 78.34 & 63.48 & 58.89 \\
\hline & 45.0 & 58.94 & 61.65 & 63.53 & 64.87 & 65.77 & 65.77 & 64.21 & 62.70 & 61.61 & 58.81 \\
\hline
\end{tabular}


Table B - 12: Temperature $\left({ }^{\circ} \mathrm{C}\right)$ map of the west side of position B at MOC2 144B (38.0 EFPD).

\begin{tabular}{|c|c|c|c|c|c|c|c|c|c|c|c|}
\hline \multirow{2}{*}{$\begin{array}{c}\text { Plate } \\
\text { Location }\end{array}$} & \multirow{2}{*}{$\begin{array}{c}\text { Axial } \\
\text { Location (in) }\end{array}$} & \multicolumn{10}{|c|}{ Width of Plate (inches) } \\
\hline & & 0.00 & 0.08 & 0.16 & 0.25 & 0.50 & 1.00 & 1.50 & 2.00 & 2.08 & 2.16 \\
\hline \multirow{9}{*}{ B-1 } & 0.0 & 51.67 & 51.78 & 51.76 & 51.76 & 51.76 & 51.76 & 51.76 & 51.76 & 51.78 & 51.67 \\
\hline & 1.0 & 53.25 & 53.88 & 75.47 & 77.84 & 77.91 & 77.91 & 77.64 & 66.18 & 53.86 & 53.13 \\
\hline & 2.0 & 53.50 & 54.23 & 78.71 & 81.34 & 81.43 & 81.43 & 81.11 & 68.24 & 54.20 & 53.08 \\
\hline & 3.0 & 53.75 & 54.45 & 79.26 & 81.98 & 82.09 & 82.09 & 81.74 & 68.66 & 54.41 & 53.04 \\
\hline & 4.0 & 54.04 & 54.85 & 82.56 & 85.54 & 85.66 & 85.66 & 85.27 & 70.80 & 54.81 & 53.36 \\
\hline & 5.0 & 54.30 & 55.25 & 85.82 & 89.05 & 89.19 & 89.19 & 88.77 & 72.92 & 55.20 & 53.48 \\
\hline & 6.0 & 54.59 & 55.70 & 89.26 & 92.76 & 92.92 & 92.92 & 92.46 & 75.19 & 55.64 & 53.68 \\
\hline & 7.0 & 54.62 & 53.33 & 56.27 & 57.21 & 57.36 & 57.36 & 56.96 & 54.96 & 53.26 & 53.72 \\
\hline & 7.5 & 54.66 & 52.79 & 53.11 & 53.34 & 53.47 & 53.47 & 53.24 & 52.89 & 52.72 & 53.73 \\
\hline \multirow{9}{*}{ B-2 } & 7.5 & 54.66 & 52.79 & 53.11 & 53.34 & 53.47 & 53.47 & 53.24 & 52.89 & 52.72 & 53.73 \\
\hline & 8.0 & 54.85 & 53.51 & 56.78 & 57.81 & 57.98 & 57.98 & 57.54 & 55.33 & 53.44 & 53.86 \\
\hline & 9.0 & 55.34 & 56.47 & 93.89 & 97.74 & 97.92 & 97.92 & 97.40 & 78.34 & 56.44 & 55.37 \\
\hline & 10.0 & 55.59 & 56.72 & 94.87 & 98.81 & 99.01 & 99.01 & 98.45 & 79.03 & 56.67 & 55.31 \\
\hline & 11.0 & 55.87 & 57.08 & 96.79 & 100.99 & 101.24 & 101.24 & 100.58 & 80.35 & 57.01 & 55.02 \\
\hline & 12.0 & 56.12 & 57.41 & 98.78 & 103.28 & 103.56 & 103.56 & 102.85 & 81.71 & 57.33 & 54.82 \\
\hline & 13.0 & 56.35 & 57.67 & 99.22 & 103.83 & 104.13 & 104.13 & 103.38 & 82.08 & 57.59 & 55.12 \\
\hline & 14.0 & 56.55 & 57.90 & 101.53 & 106.27 & 106.58 & 106.58 & 105.82 & 83.49 & 57.81 & 55.22 \\
\hline & 15.0 & 56.46 & 54.17 & 54.89 & 55.40 & 55.70 & 55.70 & 55.16 & 54.48 & 54.07 & 55.17 \\
\hline \multirow{9}{*}{ B-3 } & 15.0 & 56.46 & 54.17 & 54.89 & 55.40 & 55.70 & 55.70 & 55.16 & 54.48 & 54.07 & 55.17 \\
\hline & 16.0 & 57.04 & 58.56 & 104.63 & 109.66 & 109.99 & 109.99 & 109.17 & 85.56 & 58.45 & 55.62 \\
\hline & 17.0 & 57.24 & 58.74 & 104.20 & 109.22 & 109.57 & 109.57 & 108.71 & 85.37 & 58.67 & 56.87 \\
\hline & 18.0 & 57.45 & 58.94 & 104.40 & 109.44 & 109.82 & 109.82 & 108.92 & 85.55 & 58.89 & 57.41 \\
\hline & 19.0 & 57.67 & 59.20 & 104.89 & 109.98 & 110.38 & 110.38 & 109.44 & 85.92 & 59.13 & 57.23 \\
\hline & 20.0 & 57.86 & 59.45 & 105.61 & 110.77 & 111.18 & 111.18 & 110.21 & 86.42 & 59.34 & 56.74 \\
\hline & 21.0 & 58.07 & 59.85 & 107.79 & 113.12 & 113.55 & 113.55 & 112.54 & 87.83 & 59.74 & 56.68 \\
\hline & 22.0 & 57.96 & 56.55 & 61.39 & 63.11 & 63.59 & 63.59 & 62.57 & 59.25 & 56.45 & 56.42 \\
\hline & 22.5 & 57.93 & 55.75 & 56.79 & 57.54 & 57.99 & 57.99 & 57.18 & 56.24 & 55.69 & 58.05 \\
\hline \multirow{9}{*}{ B-4 } & 22.5 & 57.93 & 55.75 & 56.79 & 57.54 & 57.99 & 57.99 & 57.18 & 56.24 & 55.69 & 58.05 \\
\hline & 23.0 & 58.10 & 56.69 & 61.48 & 63.19 & 63.68 & 63.67 & 62.65 & 59.36 & 56.58 & 56.54 \\
\hline & 24.0 & 58.54 & 60.39 & 108.41 & 113.78 & 114.23 & 114.23 & 113.18 & 88.39 & 60.26 & 56.85 \\
\hline & 25.0 & 58.67 & 60.49 & 107.11 & 112.38 & 112.86 & 112.85 & 111.77 & 87.66 & 60.36 & 57.48 \\
\hline & 26.0 & 58.82 & 60.75 & 107.40 & 112.70 & 113.19 & 113.19 & 112.08 & 87.92 & 60.65 & 58.47 \\
\hline & 27.0 & 58.95 & 60.92 & 107.03 & 112.31 & 112.82 & 112.82 & 111.68 & 87.76 & 60.84 & 58.89 \\
\hline & 28.0 & 59.05 & 61.13 & 107.24 & 112.54 & 113.07 & 113.06 & 111.90 & 87.96 & 61.03 & 58.60 \\
\hline & 29.0 & 59.11 & 61.26 & 108.02 & 113.32 & 113.84 & 113.84 & 112.69 & 88.44 & 61.13 & 57.91 \\
\hline & 30.0 & 58.88 & 57.43 & 58.78 & 59.73 & 60.33 & 60.33 & 59.27 & 58.09 & 57.29 & 57.35 \\
\hline \multirow{9}{*}{ B-5 } & 30.0 & 58.88 & 57.43 & 58.78 & 59.74 & 60.33 & 60.33 & 59.27 & 58.09 & 57.29 & 57.35 \\
\hline & 31.0 & 59.27 & 61.44 & 105.39 & 110.50 & 111.03 & 111.02 & 109.86 & 87.00 & 61.32 & 57.94 \\
\hline & 32.0 & 59.35 & 61.49 & 103.17 & 108.13 & 108.67 & 108.67 & 107.47 & 85.72 & 61.36 & 57.80 \\
\hline & 33.0 & 59.40 & 61.63 & 103.14 & 108.07 & 108.63 & 108.62 & 107.41 & 85.75 & 61.49 & 57.74 \\
\hline & 34.0 & 59.44 & 61.76 & 102.10 & 106.94 & 107.52 & 107.52 & 106.28 & 85.19 & 61.62 & 58.24 \\
\hline & 35.0 & 59.47 & 61.81 & 100.58 & 105.30 & 105.88 & 105.88 & 104.63 & 84.33 & 61.70 & 59.01 \\
\hline & 36.0 & 59.49 & 61.99 & 100.52 & 105.23 & 105.82 & 105.82 & 104.56 & 84.37 & 61.90 & 59.20 \\
\hline & 37.0 & 59.28 & 59.47 & 63.83 & 65.60 & 66.28 & 66.27 & 64.95 & 61.91 & 59.36 & 59.02 \\
\hline & 37.5 & 59.20 & 58.88 & 60.34 & 61.38 & 62.05 & 62.04 & 60.87 & 59.63 & 58.74 & 57.81 \\
\hline \multirow{9}{*}{ B-6 } & 37.5 & 59.20 & 58.88 & 60.34 & 61.38 & 62.05 & 62.04 & 60.87 & 59.63 & 58.74 & 57.81 \\
\hline & 38.0 & 59.26 & 59.52 & 63.60 & 65.29 & 65.95 & 65.95 & 64.66 & 61.80 & 59.38 & 58.16 \\
\hline & 39.0 & 59.43 & 62.19 & 98.26 & 102.73 & 103.31 & 103.31 & 102.07 & 83.14 & 62.04 & 58.13 \\
\hline & $\begin{array}{l}40.0 \\
\end{array}$ & 59.39 & 62.10 & 95.25 & 99.46 & 100.06 & 100.05 & 98.81 & 81.36 & 61.96 & 58.01 \\
\hline & 41.0 & 59.34 & 62.02 & 92.36 & 96.25 & 96.85 & 96.85 & 95.60 & 79.62 & 61.88 & 58.14 \\
\hline & 42.0 & 59.28 & 61.96 & 89.87 & 93.46 & 94.06 & 94.06 & 92.83 & 78.10 & 61.82 & 57.92 \\
\hline & 43.0 & 59.21 & 62.00 & 89.62 & 93.13 & 93.73 & 93.73 & 92.53 & 77.97 & 61.87 & 58.26 \\
\hline & 44.0 & 59.09 & 61.86 & 86.90 & 90.15 & 90.70 & 90.70 & 89.58 & 76.29 & 61.76 & 58.72 \\
\hline & 45.0 & 58.88 & 59.91 & 61.37 & 62.39 & 63.05 & 63.05 & 61.87 & 60.70 & 59.83 & 58.73 \\
\hline
\end{tabular}


Table B - 13: Temperature $\left({ }^{\circ} \mathrm{C}\right)$ map of the east side of position A at EOC 144B (51.7 EFPD).

\begin{tabular}{|c|c|c|c|c|c|c|c|c|c|c|c|}
\hline \multirow{2}{*}{$\begin{array}{c}\text { Plate } \\
\text { Location }\end{array}$} & \multirow{2}{*}{$\begin{array}{c}\text { Axial } \\
\text { Location (in) }\end{array}$} & \multicolumn{10}{|c|}{ Width of Plate (inches) } \\
\hline & & 0.00 & 0.08 & 0.16 & 0.25 & 0.50 & 1.00 & 1.50 & 2.00 & 2.08 & 2.16 \\
\hline \multirow{9}{*}{ A-1 } & 0.0 & 51.67 & 51.77 & 51.76 & 51.76 & 51.76 & 51.76 & 51.76 & 51.76 & 51.77 & 51.67 \\
\hline & 1.0 & 52.48 & 53.83 & 75.45 & 77.82 & 77.89 & 77.89 & 77.62 & 66.16 & 53.83 & 52.47 \\
\hline & 2.0 & 52.65 & 54.17 & 78.58 & 81.20 & 81.29 & 81.29 & 80.97 & 68.16 & 54.16 & 52.61 \\
\hline & 3.0 & 52.81 & 54.37 & 79.10 & 81.81 & 81.92 & 81.92 & 81.57 & 68.55 & 54.36 & 52.76 \\
\hline & 4.0 & 53.00 & 54.73 & 82.19 & 85.14 & 85.26 & 85.26 & 84.88 & 70.55 & 54.72 & 52.96 \\
\hline & 5.0 & 53.17 & 55.06 & 84.76 & 87.92 & 88.06 & 88.06 & 87.64 & 72.23 & 55.05 & 53.13 \\
\hline & 6.0 & 53.37 & 55.51 & 88.38 & 91.83 & 91.98 & 91.98 & 91.53 & 74.61 & 55.50 & 53.32 \\
\hline & 7.0 & 53.34 & 53.18 & 56.12 & 57.04 & 57.20 & 57.20 & 56.80 & 54.84 & 53.16 & 53.27 \\
\hline & 7.5 & 53.35 & 52.65 & 53.03 & 53.27 & 53.40 & 53.40 & 53.16 & 52.82 & 52.63 & 53.27 \\
\hline \multirow{9}{*}{ A-2 } & 7.5 & 53.35 & 52.65 & 53.03 & 53.27 & 53.40 & 53.40 & 53.16 & 52.82 & 52.63 & 53.27 \\
\hline & 8.0 & 53.50 & 53.37 & 56.75 & 57.80 & 57.97 & 57.97 & 57.53 & 55.29 & 53.35 & 53.42 \\
\hline & 9.0 & 53.90 & 56.36 & 94.34 & 98.23 & 98.41 & 98.41 & 97.89 & 78.59 & 56.35 & 53.86 \\
\hline & 10.0 & 54.07 & 56.62 & 95.67 & 99.68 & 99.91 & 99.91 & 99.32 & 79.52 & 56.61 & 54.02 \\
\hline & 11.0 & 54.28 & 56.98 & 97.77 & 102.11 & 102.37 & 102.37 & 101.69 & 80.95 & 56.97 & 54.21 \\
\hline & 12.0 & 54.46 & 57.23 & 98.99 & 103.54 & 103.82 & 103.82 & 103.10 & 81.81 & 57.22 & 54.38 \\
\hline & 13.0 & 54.64 & 57.46 & 99.38 & 104.03 & 104.33 & 104.33 & 103.57 & 82.13 & 57.45 & 54.57 \\
\hline & 14.0 & 54.79 & 57.68 & 101.82 & 106.61 & 106.92 & 106.92 & 106.16 & 83.62 & 57.66 & 54.72 \\
\hline & 15.0 & 54.65 & 53.89 & 54.70 & 55.22 & 55.52 & 55.52 & 54.99 & 54.29 & 53.87 & 54.56 \\
\hline \multirow{9}{*}{ A-3 } & 15.0 & 54.65 & 53.89 & 54.70 & 55.22 & 55.52 & 55.52 & 54.99 & 54.29 & 53.87 & 54.56 \\
\hline & 16.0 & 55.12 & 57.69 & 97.92 & 102.36 & 102.68 & 102.68 & 101.89 & 81.31 & 57.66 & 55.00 \\
\hline & $\begin{array}{l}17.0 \\
\end{array}$ & 55.30 & 57.88 & 98.02 & 102.50 & 102.85 & 102.85 & 102.02 & 81.45 & 57.87 & 55.23 \\
\hline & 18.0 & 55.45 & 58.06 & 98.19 & 102.72 & 103.09 & 103.09 & 102.23 & 81.62 & 58.05 & 55.40 \\
\hline & 19.0 & 55.64 & 58.28 & 98.45 & 103.04 & 103.43 & 103.43 & 102.53 & 81.86 & 58.26 & 55.57 \\
\hline & 20.0 & 55.80 & 58.53 & 99.52 & 104.20 & 104.61 & 104.61 & 103.68 & 82.58 & 58.51 & 55.70 \\
\hline & 21.0 & 55.98 & 58.83 & 100.91 & 105.71 & 106.14 & 106.14 & 105.17 & 83.51 & 58.81 & 55.88 \\
\hline & 22.0 & 55.88 & 55.94 & 60.29 & 61.86 & 62.33 & 62.33 & 61.37 & 58.40 & 55.92 & 55.79 \\
\hline & 22.5 & 55.85 & 55.24 & 56.27 & 56.99 & 57.43 & 57.43 & 56.66 & 55.77 & 55.22 & 55.79 \\
\hline \multirow{9}{*}{ A-4 } & 22.5 & 55.85 & 55.24 & 56.27 & 56.99 & 57.43 & 57.43 & 56.66 & 55.77 & 55.22 & 55.79 \\
\hline & 23.0 & 56.01 & 56.13 & 60.76 & 62.40 & 62.87 & 62.87 & 61.89 & 58.76 & 56.11 & 55.92 \\
\hline & 24.0 & 56.41 & 59.66 & 105.54 & 110.71 & 111.15 & 111.15 & 110.14 & 86.51 & 59.63 & 56.29 \\
\hline & 25.0 & 56.54 & 59.77 & 104.68 & 109.79 & 110.25 & 110.25 & 109.20 & 86.04 & 59.75 & 56.43 \\
\hline & 26.0 & 56.69 & 59.99 & 104.85 & 109.98 & 110.47 & 110.47 & 109.38 & 86.22 & 59.97 & 56.61 \\
\hline & 27.0 & 56.81 & 60.11 & 104.18 & 109.27 & 109.77 & 109.77 & 108.66 & 85.87 & 60.10 & 56.75 \\
\hline & 28.0 & 56.93 & 60.29 & 104.35 & 109.45 & 109.97 & 109.97 & 108.84 & 86.04 & 60.28 & 56.85 \\
\hline & 29.0 & 57.01 & 60.40 & 105.03 & 110.14 & 110.65 & 110.65 & 109.54 & 86.46 & 60.38 & 56.90 \\
\hline & 30.0 & 56.83 & 56.73 & 58.08 & 59.01 & 59.59 & 59.59 & 58.58 & 57.45 & 56.70 & 56.71 \\
\hline \multirow{9}{*}{ A-5 } & 30.0 & 56.83 & 56.73 & 58.08 & 59.01 & 59.59 & 59.59 & 58.58 & 57.46 & 56.70 & 56.71 \\
\hline & 31.0 & 57.22 & 60.78 & 105.17 & 110.30 & 110.82 & 110.82 & 109.68 & 86.70 & 60.76 & 57.13 \\
\hline & 32.0 & 57.33 & 60.86 & 103.56 & 108.59 & 109.13 & 109.13 & 107.95 & 85.79 & 60.84 & 57.24 \\
\hline & 33.0 & 57.42 & 61.00 & 103.57 & 108.58 & 109.14 & 109.14 & 107.94 & 85.84 & 60.98 & 57.30 \\
\hline & 34.0 & 57.50 & 61.12 & 102.70 & 107.65 & 108.22 & 108.22 & 107.01 & 85.38 & 61.10 & 57.39 \\
\hline & 35.0 & 57.57 & 61.17 & 101.42 & 106.26 & 106.84 & 106.84 & 105.61 & 84.65 & 61.16 & 57.49 \\
\hline & 36.0 & 57.64 & 61.26 & 100.33 & 105.08 & 105.67 & 105.67 & 104.43 & 84.05 & 61.25 & 57.58 \\
\hline & 37.0 & 57.50 & 58.67 & 63.12 & 64.90 & 65.56 & 65.56 & 64.27 & 61.22 & 58.65 & 57.42 \\
\hline & 37.5 & 57.44 & 58.07 & 59.56 & 60.59 & 61.24 & 61.24 & 60.10 & 58.90 & 58.04 & 57.33 \\
\hline \multirow{9}{*}{ A-6 } & 37.5 & 57.44 & 58.07 & 59.56 & 60.59 & 61.24 & 61.24 & 60.10 & 58.90 & 58.04 & 57.33 \\
\hline & 38.0 & 57.53 & 58.69 & 62.78 & 64.47 & 65.12 & 65.12 & 63.86 & 61.04 & 58.67 & 57.42 \\
\hline & 39.0 & 57.75 & 61.34 & 97.08 & 101.51 & 102.10 & 102.10 & 100.88 & 82.20 & 61.32 & 57.65 \\
\hline & $\begin{array}{l}40.0 \\
\end{array}$ & 57.77 & 61.27 & 94.43 & 98.61 & 99.21 & 99.21 & 97.98 & 80.62 & 61.25 & 57.69 \\
\hline & 41.0 & 57.79 & 61.20 & 91.86 & 95.71 & 96.32 & 96.32 & 95.09 & 79.05 & 61.19 & 57.71 \\
\hline & 42.0 & 57.81 & 61.15 & 89.53 & 93.13 & 93.71 & 93.71 & 92.55 & 77.63 & 61.13 & 57.71 \\
\hline & 43.0 & 57.81 & 61.18 & 89.27 & 92.80 & 93.37 & 93.37 & 92.24 & 77.50 & 61.17 & 57.72 \\
\hline & 44.0 & 57.77 & 61.02 & 86.34 & 89.61 & 90.16 & 90.17 & 89.06 & 75.67 & 61.01 & 57.72 \\
\hline & 45.0 & 57.60 & 59.04 & 60.50 & 61.52 & 62.18 & 62.18 & 61.03 & 59.89 & 59.03 & 57.57 \\
\hline
\end{tabular}


Table B - 14: Temperature $\left({ }^{\circ} \mathrm{C}\right)$ map of the west side of position A at EOC 144B (51.7 EFPD).

\begin{tabular}{|c|c|c|c|c|c|c|c|c|c|c|c|}
\hline \multirow{2}{*}{$\begin{array}{c}\text { Plate } \\
\text { Location }\end{array}$} & \multirow{2}{*}{$\begin{array}{c}\text { Axial } \\
\text { Location (in) }\end{array}$} & \multicolumn{10}{|c|}{ Width of Plate (inches) } \\
\hline & & 0.00 & 0.08 & 0.16 & 0.25 & 0.50 & 1.00 & 1.50 & 2.00 & 2.08 & 2.16 \\
\hline \multirow{9}{*}{ A-1 } & 0.0 & 51.67 & 51.76 & 51.76 & 51.76 & 51.76 & 51.76 & 51.76 & 51.76 & 51.76 & 51.67 \\
\hline & 1.0 & 52.60 & 53.83 & 75.46 & 77.83 & 77.90 & 77.90 & 77.63 & 66.17 & 53.83 & 52.57 \\
\hline & 2.0 & 52.78 & 54.18 & 78.61 & 81.23 & 81.33 & 81.33 & 81.01 & 68.18 & 54.17 & 52.71 \\
\hline & 3.0 & 52.96 & 54.39 & 79.16 & 81.88 & 82.00 & 82.00 & 81.63 & 68.60 & 54.39 & 52.87 \\
\hline & 4.0 & 53.17 & 54.78 & 82.29 & 85.26 & 85.40 & 85.40 & 84.99 & 70.62 & 54.77 & 53.10 \\
\hline & 5.0 & 53.36 & 55.13 & 84.90 & 88.09 & 88.25 & 88.25 & 87.79 & 72.33 & 55.12 & 53.27 \\
\hline & 6.0 & 53.59 & 55.61 & 88.57 & 92.06 & 92.24 & 92.24 & 91.73 & 74.75 & 55.60 & 53.47 \\
\hline & 7.0 & 53.53 & 53.30 & 56.31 & 57.28 & 57.46 & 57.46 & 57.02 & 55.01 & 53.29 & 53.40 \\
\hline & 7.5 & 53.53 & 52.78 & 53.24 & 53.53 & 53.69 & 53.69 & 53.40 & 53.01 & 52.77 & 53.38 \\
\hline \multirow{9}{*}{ A-2 } & 7.5 & 53.53 & 52.78 & 53.24 & 53.53 & 53.69 & 53.69 & 53.40 & 53.01 & 52.77 & 53.38 \\
\hline & 8.0 & 53.70 & 53.52 & 56.98 & 58.07 & 58.27 & 58.27 & 57.78 & 55.48 & 53.51 & 53.55 \\
\hline & 9.0 & 54.16 & 56.53 & 94.63 & 98.58 & 98.81 & 98.81 & 98.22 & 78.83 & 56.52 & 54.10 \\
\hline & 10.0 & 54.36 & 56.82 & 96.01 & 100.15 & 100.44 & 100.44 & 99.73 & 79.79 & 56.81 & 54.26 \\
\hline & 11.0 & 54.59 & 57.22 & 98.16 & 102.67 & 102.99 & 102.99 & 102.21 & 81.27 & 57.21 & 54.44 \\
\hline & 12.0 & 54.78 & 57.51 & 99.50 & 104.17 & 104.53 & 104.53 & 103.69 & 82.17 & 57.50 & 54.61 \\
\hline & 13.0 & 54.98 & 57.78 & 99.98 & 104.74 & 105.13 & 105.13 & 104.23 & 82.54 & 57.77 & 54.83 \\
\hline & 14.0 & 55.15 & 58.04 & 102.49 & 107.41 & 107.81 & 107.81 & 106.89 & 84.08 & 58.03 & 54.98 \\
\hline & 15.0 & 54.96 & 54.29 & 55.29 & 55.92 & 56.30 & 56.30 & 55.63 & 54.80 & 54.28 & 54.76 \\
\hline \multirow{9}{*}{ A-3 } & 15.0 & 54.96 & 54.29 & 55.29 & 55.92 & 56.30 & 56.30 & 55.63 & 54.80 & 54.28 & 54.76 \\
\hline & 16.0 & 55.51 & 58.12 & 98.66 & 103.30 & 103.73 & 103.73 & 102.77 & 81.86 & 58.10 & 55.27 \\
\hline & 17.0 & 55.70 & 58.35 & 98.84 & 103.52 & 103.98 & 103.98 & 102.96 & 82.04 & 58.34 & 55.57 \\
\hline & 18.0 & 55.88 & 58.58 & 99.10 & 103.82 & 104.31 & 104.31 & 103.24 & 82.26 & 58.56 & 55.78 \\
\hline & 19.0 & 56.08 & 58.85 & 99.47 & 104.24 & 104.76 & 104.76 & 103.64 & 82.56 & 58.83 & 55.94 \\
\hline & 20.0 & 56.25 & 59.15 & 100.63 & 105.50 & 106.04 & 106.04 & 104.88 & 83.34 & 59.13 & 56.05 \\
\hline & 21.0 & 56.45 & 59.51 & 102.11 & 107.11 & 107.68 & 107.68 & 106.47 & 84.33 & 59.49 & 56.24 \\
\hline & 22.0 & 56.31 & 56.67 & 61.29 & 63.03 & 63.62 & 63.62 & 62.45 & 59.29 & 56.65 & 56.12 \\
\hline & 22.5 & 56.27 & 55.99 & 57.31 & 58.21 & 58.78 & 58.78 & 57.79 & 56.69 & 55.98 & 56.17 \\
\hline \multirow{9}{*}{ A-4 } & 22.5 & 56.27 & 55.99 & 57.31 & 58.21 & 58.78 & 58.78 & 57.79 & 56.69 & 55.98 & 56.17 \\
\hline & 23.0 & 56.45 & 56.90 & 61.80 & 63.62 & 64.22 & 64.22 & 63.02 & 59.69 & 56.89 & 56.26 \\
\hline & 24.0 & 56.92 & 60.46 & 106.92 & 112.30 & 112.91 & 112.91 & 111.62 & 87.46 & 60.44 & 56.68 \\
\hline & 25.0 & 57.06 & 60.63 & 106.13 & 111.46 & 112.10 & 112.10 & 110.76 & 87.05 & 60.60 & 56.83 \\
\hline & 26.0 & 57.23 & 60.91 & 106.39 & 111.76 & 112.42 & 112.42 & 111.04 & 87.29 & 60.88 & 57.07 \\
\hline & 27.0 & 57.36 & 61.09 & 105.81 & 111.15 & 111.83 & 111.83 & 110.41 & 87.01 & 61.06 & 57.24 \\
\hline & 28.0 & 57.49 & 61.32 & 106.06 & 111.42 & 112.13 & 112.13 & 110.67 & 87.24 & 61.30 & 57.33 \\
\hline & 29.0 & 57.57 & 61.49 & 106.83 & 112.20 & 112.92 & 112.92 & 111.46 & 87.72 & 61.47 & 57.34 \\
\hline & 30.0 & 57.33 & 57.88 & 59.59 & 60.76 & 61.51 & 61.51 & 60.20 & 58.81 & 57.86 & 57.10 \\
\hline \multirow{9}{*}{ A-5 } & 30.0 & 57.33 & 57.88 & 59.59 & 60.76 & 61.51 & 61.51 & 60.20 & 58.81 & 57.86 & 57.10 \\
\hline & 31.0 & 57.80 & 61.96 & 107.09 & 112.49 & 113.22 & 113.22 & 111.73 & 88.05 & 61.93 & 57.60 \\
\hline & 32.0 & 57.92 & 62.09 & 105.55 & 110.86 & 111.61 & 111.61 & 110.08 & 87.20 & 62.06 & 57.71 \\
\hline & 33.0 & 58.01 & 62.28 & 105.63 & 110.93 & 111.70 & 111.70 & 110.14 & 87.31 & 62.25 & 57.77 \\
\hline & 34.0 & 58.11 & 62.46 & 104.86 & 110.10 & 110.89 & 110.89 & 109.30 & 86.92 & 62.43 & 57.87 \\
\hline & 35.0 & 58.18 & 62.57 & 103.65 & 108.79 & 109.60 & 109.60 & 107.99 & 86.25 & 62.54 & 58.01 \\
\hline & 36.0 & 58.26 & 62.71 & 102.65 & 107.71 & 108.53 & 108.53 & 106.90 & 85.72 & 62.69 & 58.12 \\
\hline & 37.0 & 58.07 & 60.19 & 65.00 & 67.04 & 67.90 & 67.90 & 66.28 & 62.95 & 60.16 & 57.91 \\
\hline & 37.5 & 58.00 & 59.60 & 61.49 & 62.78 & 63.63 & 63.63 & 62.16 & 60.65 & 59.58 & 57.77 \\
\hline \multirow{9}{*}{ A-6 } & 37.5 & 58.00 & 59.60 & 61.49 & 62.78 & 63.63 & 63.63 & 62.16 & 60.65 & 59.58 & 57.77 \\
\hline & 38.0 & 58.10 & 60.25 & 64.70 & 66.65 & 67.49 & 67.49 & 65.91 & 62.80 & 60.22 & 57.88 \\
\hline & 39.0 & 58.37 & 62.92 & 99.58 & 104.33 & 105.15 & 105.15 & 103.53 & 84.01 & 62.89 & 58.15 \\
\hline & 40.0 & 58.40 & 62.89 & 96.98 & 101.51 & 102.35 & 102.35 & 100.72 & 82.49 & 62.87 & 58.20 \\
\hline & 41.0 & 58.41 & 62.88 & 94.42 & 98.71 & 99.55 & 99.55 & 97.92 & 80.99 & 62.85 & 58.22 \\
\hline & 42.0 & 58.42 & 62.88 & 92.09 & 96.18 & 97.03 & 97.03 & 95.40 & 79.64 & 62.84 & 58.21 \\
\hline & 43.0 & 58.43 & 62.95 & 91.89 & 95.91 & 96.75 & 96.75 & 95.14 & 79.54 & 62.92 & 58.22 \\
\hline & 44.0 & 58.38 & 62.84 & 88.97 & 92.71 & 93.55 & 93.55 & 91.97 & 77.79 & 62.81 & 58.25 \\
\hline & 45.0 & 58.17 & 60.89 & 62.74 & 64.03 & 64.89 & 64.89 & 63.40 & 61.95 & 60.87 & 58.09 \\
\hline
\end{tabular}


Table B - 15: Temperature $\left({ }^{\circ} \mathrm{C}\right)$ map of the east side of position B at EOC 144B (51.7 EFPD).

\begin{tabular}{|c|c|c|c|c|c|c|c|c|c|c|c|}
\hline \multirow{2}{*}{$\begin{array}{c}\text { Plate } \\
\text { Location }\end{array}$} & \multirow{2}{*}{$\begin{array}{c}\text { Axial } \\
\text { Location (in) }\end{array}$} & \multicolumn{10}{|c|}{ Width of Plate (inches) } \\
\hline & & 0.00 & 0.08 & 0.16 & 0.25 & 0.50 & 1.00 & 1.50 & 2.00 & 2.08 & 2.16 \\
\hline \multirow{9}{*}{ B-1 } & 0.0 & 51.67 & 51.79 & 51.76 & 51.76 & 51.76 & 51.76 & 51.76 & 51.76 & 51.79 & 51.67 \\
\hline & 1.0 & 52.94 & 53.86 & 75.02 & 77.35 & 77.42 & 77.42 & 77.15 & 65.90 & 53.84 & 52.84 \\
\hline & 2.0 & 53.16 & 54.21 & 78.09 & 80.67 & 80.76 & 80.76 & 80.45 & 67.85 & 54.16 & 52.91 \\
\hline & 3.0 & 53.37 & 54.44 & 78.64 & 81.32 & 81.43 & 81.43 & 81.07 & 68.27 & 54.38 & 53.01 \\
\hline & 4.0 & 53.63 & 54.84 & 81.79 & 84.72 & 84.86 & 84.86 & 84.45 & 70.31 & 54.78 & 53.29 \\
\hline & 5.0 & 53.86 & 55.24 & 84.88 & 88.07 & 88.23 & 88.23 & 87.77 & 72.33 & 55.17 & 53.45 \\
\hline & 6.0 & 54.13 & 55.69 & 88.06 & 91.50 & 91.69 & 91.69 & 91.18 & 74.43 & 55.60 & 53.64 \\
\hline & 7.0 & 54.10 & 53.43 & 56.28 & 57.23 & 57.41 & 57.41 & 56.97 & 54.99 & 53.34 & 53.59 \\
\hline & 7.5 & 54.12 & 52.93 & 53.26 & 53.53 & 53.69 & 53.69 & 53.41 & 53.02 & 52.83 & 53.58 \\
\hline \multirow{9}{*}{ B-2 } & 7.5 & 54.12 & 52.93 & 53.26 & 53.53 & 53.69 & 53.69 & 53.41 & 53.02 & 52.83 & 53.58 \\
\hline & 8.0 & 54.30 & 53.62 & 56.77 & 57.81 & 58.01 & 58.01 & 57.53 & 55.36 & 53.53 & 53.74 \\
\hline & 9.0 & 54.80 & 56.47 & 92.43 & 96.21 & 96.43 & 96.43 & 95.85 & 77.40 & 56.44 & 54.70 \\
\hline & 10.0 & 55.02 & 56.74 & 93.44 & 97.31 & 97.56 & 97.56 & 96.94 & 78.12 & 56.68 & 54.78 \\
\hline & 11.0 & 55.28 & 57.12 & 95.21 & 99.28 & 99.59 & 99.59 & 98.84 & 79.35 & 57.01 & 54.79 \\
\hline & 12.0 & 55.51 & 57.46 & 97.04 & 101.39 & 101.74 & 101.74 & 100.93 & 80.60 & 57.35 & 54.83 \\
\hline & 13.0 & 55.73 & 57.74 & 97.48 & 101.97 & 102.35 & 102.35 & 101.47 & 80.98 & 57.63 & 55.10 \\
\hline & 14.0 & 55.92 & 57.97 & 99.40 & 104.05 & 104.44 & 104.44 & 103.55 & 82.18 & 57.86 & 55.24 \\
\hline & 15.0 & 55.76 & 54.51 & 55.30 & 55.91 & 56.29 & 56.29 & 55.63 & 54.83 & 54.37 & 55.05 \\
\hline \multirow{9}{*}{ B-3 } & 15.0 & 55.76 & 54.51 & 55.30 & 55.91 & 56.29 & 56.29 & 55.63 & 54.83 & 54.37 & 55.05 \\
\hline & 16.0 & 56.35 & 58.64 & 102.24 & 107.16 & 107.59 & 107.59 & 106.62 & 84.09 & 58.51 & 55.55 \\
\hline & 17.0 & 56.56 & 58.86 & 102.07 & 107.01 & 107.47 & 107.47 & 106.44 & 84.06 & 58.77 & 56.24 \\
\hline & 18.0 & 56.75 & 59.09 & 102.33 & 107.30 & 107.79 & 107.79 & 106.71 & 84.29 & 59.05 & 56.59 \\
\hline & 19.0 & 56.98 & 59.38 & 102.85 & 107.88 & 108.40 & 108.40 & 107.27 & 84.68 & 59.29 & 56.61 \\
\hline & 20.0 & 57.16 & 59.64 & 103.44 & 108.53 & 109.07 & 109.07 & 107.90 & 85.11 & 59.50 & 56.50 \\
\hline & 21.0 & 57.37 & 60.04 & 105.21 & 110.45 & 111.02 & 111.02 & 109.80 & 86.27 & 59.90 & 56.62 \\
\hline & 22.0 & 57.23 & 57.01 & 61.71 & 63.50 & 64.09 & 64.09 & 62.90 & 59.60 & 56.87 & 56.45 \\
\hline & 22.5 & 57.19 & 56.28 & 57.45 & 58.34 & 58.91 & 58.91 & 57.92 & 56.82 & 56.23 & 57.07 \\
\hline \multirow{9}{*}{ B-4 } & 22.5 & 57.19 & 56.28 & 57.45 & 58.34 & 58.91 & 58.91 & 57.92 & 56.82 & 56.23 & 57.07 \\
\hline & 23.0 & 57.38 & 57.17 & 61.85 & 63.64 & 64.24 & 64.23 & 63.04 & 59.75 & 57.03 & 56.58 \\
\hline & 24.0 & 57.85 & 60.67 & 106.18 & 111.49 & 112.09 & 112.09 & 110.81 & 87.05 & 60.51 & 56.94 \\
\hline & 25.0 & 57.99 & 60.81 & 105.13 & 110.37 & 111.00 & 111.00 & 109.67 & 86.48 & 60.66 & 57.29 \\
\hline & 26.0 & 58.15 & 61.11 & 105.56 & 110.85 & 111.51 & 111.51 & 110.13 & 86.83 & 61.00 & 57.81 \\
\hline & 27.0 & 58.28 & 61.33 & 105.45 & 110.74 & 111.42 & 111.42 & 110.01 & 86.84 & 61.27 & 58.09 \\
\hline & 28.0 & 58.41 & 61.58 & 105.72 & 111.04 & 111.75 & 111.74 & 110.29 & 87.08 & 61.47 & 58.03 \\
\hline & 29.0 & 58.48 & 61.74 & 106.31 & 111.63 & 112.34 & 112.34 & 110.89 & 87.45 & 61.59 & 57.78 \\
\hline & 30.0 & 58.23 & 58.19 & 59.73 & 60.88 & 61.62 & 61.62 & 60.32 & 58.95 & 58.03 & 57.40 \\
\hline \multirow{9}{*}{ B-5 } & 30.0 & 58.23 & 58.19 & 59.73 & 60.88 & 61.62 & 61.62 & 60.32 & 58.95 & 58.03 & 57.40 \\
\hline & 31.0 & 58.68 & 61.98 & 103.94 & 109.07 & 109.80 & 109.80 & 108.32 & 86.18 & 61.85 & 57.98 \\
\hline & 32.0 & 58.78 & 62.09 & 102.27 & 107.29 & 108.04 & 108.04 & 106.52 & 85.24 & 61.95 & 58.00 \\
\hline & 33.0 & 58.86 & 62.28 & 102.33 & 107.34 & 108.11 & 108.11 & 106.57 & 85.34 & 62.13 & 57.99 \\
\hline & 34.0 & 58.93 & 62.46 & 101.49 & 106.44 & 107.22 & 107.22 & 105.65 & 84.91 & 62.30 & 58.23 \\
\hline & 35.0 & 58.99 & 62.56 & 100.32 & 105.17 & 105.97 & 105.97 & 104.37 & 84.27 & 62.46 & 58.61 \\
\hline & 36.0 & 59.04 & 62.79 & 100.34 & 105.18 & 106.00 & 106.00 & 104.38 & 84.36 & 62.73 & 58.76 \\
\hline & 37.0 & 58.83 & 60.43 & 64.90 & 66.87 & 67.72 & 67.72 & 66.12 & 62.94 & 60.33 & 58.53 \\
\hline & 37.5 & 58.75 & 59.89 & 61.62 & 62.89 & 63.72 & 63.72 & 62.26 & 60.79 & 59.75 & 58.00 \\
\hline \multirow{9}{*}{ B-6 } & 37.5 & 58.75 & 59.89 & 61.62 & 62.89 & 63.72 & 63.72 & 62.26 & 60.79 & 59.75 & 58.00 \\
\hline & 38.0 & 58.84 & 60.52 & 64.74 & 66.63 & 67.47 & 67.46 & 65.90 & 62.88 & 60.37 & 58.20 \\
\hline & 39.0 & 59.07 & 63.10 & 98.55 & 103.19 & 104.00 & 104.00 & 102.40 & 83.44 & 62.96 & 58.37 \\
\hline & $\begin{array}{l}40.0 \\
\end{array}$ & 59.07 & 63.07 & 95.99 & 100.41 & 101.24 & 101.24 & 99.62 & 81.94 & 62.94 & 58.38 \\
\hline & 41.0 & 59.05 & 63.04 & 93.30 & 97.46 & 98.30 & 98.30 & 96.68 & 80.37 & 62.92 & 58.43 \\
\hline & 42.0 & 59.03 & 63.04 & 91.01 & 94.98 & 95.82 & 95.82 & 94.21 & 79.03 & 62.90 & 58.33 \\
\hline & 43.0 & 59.00 & 63.11 & 90.84 & 94.74 & 95.58 & 95.58 & 93.98 & 78.96 & 62.99 & 58.45 \\
\hline & 44.0 & 58.92 & 63.02 & 88.27 & 91.91 & 92.74 & 92.74 & 91.17 & 77.42 & 62.94 & 58.65 \\
\hline & 45.0 & 58.70 & 61.14 & 62.90 & 64.17 & 65.01 & 65.01 & 63.54 & 62.12 & 61.09 & 58.57 \\
\hline
\end{tabular}


Table B - 16: Temperature $\left({ }^{\circ} \mathrm{C}\right)$ map of the west side of position B at EOC 144B (51.7 EFPD).

\begin{tabular}{|c|c|c|c|c|c|c|c|c|c|c|c|}
\hline \multirow{2}{*}{$\begin{array}{c}\text { Plate } \\
\text { Location }\end{array}$} & \multirow{2}{*}{$\begin{array}{c}\text { Axial } \\
\text { Location (in) }\end{array}$} & \multicolumn{10}{|c|}{ Width of Plate (inches) } \\
\hline & & 0.00 & 0.08 & 0.16 & 0.25 & 0.50 & 1.00 & 1.50 & 2.00 & 2.08 & 2.16 \\
\hline \multirow{9}{*}{ B-1 } & 0.0 & 51.67 & 51.78 & 51.76 & 51.76 & 51.76 & 51.76 & 51.76 & 51.76 & 51.78 & 51.67 \\
\hline & 1.0 & 53.25 & 53.84 & 75.01 & 77.33 & 77.40 & 77.40 & 77.13 & 65.89 & 53.82 & 53.13 \\
\hline & 2.0 & 53.50 & 54.18 & 78.06 & 80.63 & 80.72 & 80.72 & 80.41 & 67.83 & 54.14 & 53.07 \\
\hline & 3.0 & 53.74 & 54.39 & 78.58 & 81.25 & 81.35 & 81.35 & 81.01 & 68.23 & 54.35 & 53.03 \\
\hline & 4.0 & 54.03 & 54.76 & 81.70 & 84.61 & 84.73 & 84.73 & 84.35 & 70.25 & 54.72 & 53.35 \\
\hline & 5.0 & 54.29 & 55.14 & 84.76 & 87.91 & 88.05 & 88.05 & 87.63 & 72.24 & 55.10 & 53.47 \\
\hline & 6.0 & 54.58 & 55.56 & 87.89 & 91.29 & 91.45 & 91.45 & 90.99 & 74.31 & 55.50 & 53.67 \\
\hline & 7.0 & 54.61 & 53.28 & 56.10 & 57.01 & 57.16 & 57.16 & 56.77 & 54.84 & 53.22 & 53.71 \\
\hline & 7.5 & 54.65 & 52.77 & 53.07 & 53.29 & 53.42 & 53.42 & 53.19 & 52.86 & 52.70 & 53.72 \\
\hline \multirow{9}{*}{ B-2 } & 7.5 & 54.65 & 52.77 & 53.07 & 53.29 & 53.42 & 53.42 & 53.19 & 52.86 & 52.70 & 53.72 \\
\hline & 8.0 & 54.84 & 53.46 & 56.57 & 57.56 & 57.72 & 57.72 & 57.30 & 55.19 & 53.39 & 53.85 \\
\hline & 9.0 & 55.32 & 56.29 & 92.16 & 95.88 & 96.05 & 96.05 & 95.55 & 77.20 & 56.26 & 55.35 \\
\hline & 10.0 & 55.56 & 56.53 & 93.13 & 96.93 & 97.13 & 97.12 & 96.59 & 77.89 & 56.49 & 55.29 \\
\hline & 11.0 & 55.84 & 56.87 & 94.84 & 98.78 & 99.00 & 99.00 & 98.42 & 79.07 & 56.79 & 54.99 \\
\hline & 12.0 & 56.08 & 57.17 & 96.62 & 100.79 & 101.06 & 101.06 & 100.37 & 80.28 & 57.09 & 54.79 \\
\hline & 13.0 & 56.31 & 57.41 & 97.01 & 101.28 & 101.57 & 101.57 & 100.84 & 80.61 & 57.33 & 55.08 \\
\hline & 14.0 & 56.51 & 57.61 & 98.79 & 103.28 & 103.57 & 103.57 & 102.85 & 81.77 & 57.52 & 55.18 \\
\hline & 15.0 & 56.42 & 54.10 & 54.76 & 55.25 & 55.53 & 55.53 & 55.02 & 54.37 & 54.00 & 55.15 \\
\hline \multirow{9}{*}{ B-3 } & 15.0 & 56.42 & 54.10 & 54.76 & 55.25 & 55.53 & 55.53 & 55.02 & 54.37 & 54.00 & 55.15 \\
\hline & 16.0 & 56.98 & 58.22 & 101.51 & 106.28 & 106.60 & 106.60 & 105.81 & 83.61 & 58.11 & 55.57 \\
\hline & 17.0 & 57.18 & 58.41 & 101.29 & 106.07 & 106.41 & 106.40 & 105.57 & 83.55 & 58.34 & 56.81 \\
\hline & 18.0 & 57.38 & 58.61 & 101.50 & 106.30 & 106.65 & 106.65 & 105.79 & 83.73 & 58.56 & 57.35 \\
\hline & 19.0 & 57.60 & 58.85 & 101.94 & 106.79 & 107.17 & 107.16 & 106.26 & 84.07 & 58.78 & 57.16 \\
\hline & 20.0 & 57.78 & 59.08 & 102.47 & 107.36 & 107.76 & 107.76 & 106.82 & 84.45 & 58.96 & 56.67 \\
\hline & 21.0 & 57.98 & 59.42 & 104.17 & 109.20 & 109.61 & 109.61 & 108.65 & 85.57 & 59.31 & 56.61 \\
\hline & 22.0 & 57.88 & 56.34 & 60.84 & 62.45 & 62.91 & 62.90 & 61.94 & 58.84 & 56.24 & 56.35 \\
\hline & 22.5 & 57.85 & 55.60 & 56.55 & 57.25 & 57.68 & 57.68 & 56.91 & 56.03 & 55.54 & 57.97 \\
\hline \multirow{9}{*}{ B-4 } & 22.5 & 57.85 & 55.60 & 56.55 & 57.25 & 57.68 & 57.68 & 56.91 & 56.03 & 55.54 & 57.97 \\
\hline & 23.0 & 58.02 & 56.48 & 60.94 & 62.55 & 63.01 & 63.01 & 62.04 & 58.96 & 56.37 & 56.47 \\
\hline & 24.0 & 58.43 & 59.95 & 104.98 & 110.06 & 110.49 & 110.49 & 109.49 & 86.23 & 59.82 & 56.76 \\
\hline & 25.0 & 58.55 & 60.06 & 103.87 & 108.87 & 109.32 & 109.32 & 108.29 & 85.61 & 59.93 & 57.39 \\
\hline & 26.0 & 58.69 & 60.31 & 104.22 & 109.26 & 109.73 & 109.72 & 108.66 & 85.91 & 60.21 & 58.36 \\
\hline & 27.0 & 58.82 & 60.48 & 104.04 & 109.06 & 109.54 & 109.54 & 108.45 & 85.86 & 60.41 & 58.77 \\
\hline & 28.0 & 58.92 & 60.68 & 104.23 & 109.27 & 109.77 & 109.77 & 108.66 & 86.04 & 60.58 & 58.48 \\
\hline & 29.0 & 58.97 & 60.79 & 104.74 & 109.77 & 110.27 & 110.26 & 109.17 & 86.36 & 60.65 & 57.79 \\
\hline & 30.0 & 58.75 & 57.18 & 58.42 & 59.31 & 59.87 & 59.87 & 58.87 & 57.78 & 57.05 & 57.25 \\
\hline \multirow{9}{*}{ B-5 } & 30.0 & 58.75 & 57.18 & 58.42 & 59.31 & 59.87 & 59.87 & 58.88 & 57.78 & 57.05 & 57.25 \\
\hline & 31.0 & 59.07 & 60.82 & 102.22 & 107.01 & 107.51 & 107.50 & 106.42 & 84.91 & 60.70 & 57.48 \\
\hline & 32.0 & 59.19 & 61.03 & 100.51 & 105.23 & 105.75 & 105.75 & 104.61 & 84.01 & 60.90 & 57.68 \\
\hline & 33.0 & 59.24 & 61.17 & 100.51 & 105.21 & 105.74 & 105.74 & 104.58 & 84.05 & 61.03 & 57.61 \\
\hline & 34.0 & 59.27 & 61.29 & 99.58 & 104.20 & 104.75 & 104.74 & 103.57 & 83.55 & 61.15 & 58.10 \\
\hline & 35.0 & 59.30 & 61.35 & 98.33 & 102.84 & 103.40 & 103.39 & 102.21 & 82.85 & 61.25 & 58.85 \\
\hline & 36.0 & 59.31 & 61.53 & 98.27 & 102.77 & 103.33 & 103.33 & 102.13 & 82.89 & 61.44 & 59.03 \\
\hline & 37.0 & 59.10 & 59.11 & 63.23 & 64.91 & 65.55 & 65.54 & 64.29 & 61.41 & 59.01 & 58.85 \\
\hline & 37.5 & 59.02 & 58.55 & 59.90 & 60.88 & 61.50 & 61.49 & 60.39 & 59.24 & 58.41 & 57.67 \\
\hline \multirow{9}{*}{ B-6 } & 37.5 & 59.02 & 58.55 & 59.90 & 60.88 & 61.50 & 61.49 & 60.39 & 59.24 & 58.41 & 57.67 \\
\hline & 38.0 & 59.07 & 59.16 & 63.03 & 64.64 & 65.26 & 65.26 & 64.04 & 61.32 & 59.03 & 58.01 \\
\hline & 39.0 & 59.23 & 61.72 & 96.32 & 100.60 & 101.16 & 101.15 & 99.98 & 81.83 & 61.59 & 57.97 \\
\hline & 40.0 & 59.19 & 61.66 & 93.72 & 97.75 & 98.32 & 98.31 & 97.12 & 80.28 & 61.53 & 57.85 \\
\hline & 41.0 & 59.13 & 61.58 & 91.03 & 94.71 & 95.28 & 95.28 & 94.09 & 78.63 & 61.45 & 57.98 \\
\hline & 42.0 & 59.07 & 61.53 & 88.74 & 92.19 & 92.72 & 92.72 & 91.62 & 77.24 & 61.40 & 57.76 \\
\hline & 43.0 & 59.00 & 61.58 & 88.50 & 91.90 & 92.43 & 92.42 & 91.33 & 77.12 & 61.44 & 58.08 \\
\hline & 44.0 & 58.87 & 61.44 & 85.90 & 89.06 & 89.59 & 89.58 & 88.51 & 75.52 & 61.34 & 58.51 \\
\hline & 45.0 & 58.66 & 59.53 & 60.90 & 61.86 & 62.48 & 62.48 & 61.37 & 60.27 & 59.45 & 58.52 \\
\hline
\end{tabular}


Table B - 17: Temperature $\left({ }^{\circ} \mathrm{C}\right)$ map of the east side of position A at BOC 145A (total 51.7 EFPD).

\begin{tabular}{|c|c|c|c|c|c|c|c|c|c|c|c|}
\hline \multirow{2}{*}{$\begin{array}{c}\text { Plate } \\
\text { Location }\end{array}$} & \multirow{2}{*}{$\begin{array}{c}\text { Axial } \\
\text { Location (in) }\end{array}$} & \multicolumn{10}{|c|}{ Width of Plate (inches) } \\
\hline & & 0.00 & 0.08 & 0.16 & 0.25 & 0.50 & 1.00 & 1.50 & 2.00 & 2.08 & 2.16 \\
\hline \multirow{9}{*}{ A-1 } & 0.0 & 51.67 & 51.77 & 51.76 & 51.76 & 51.77 & 51.77 & 51.76 & 51.76 & 51.77 & 51.67 \\
\hline & 1.0 & 52.49 & 53.65 & 73.26 & 75.44 & 75.50 & 75.50 & 75.25 & 64.79 & 53.64 & 52.47 \\
\hline & 2.0 & 52.65 & 53.95 & 76.13 & 78.54 & 78.63 & 78.63 & 78.33 & 66.61 & 53.95 & 52.62 \\
\hline & 3.0 & 52.81 & 54.14 & 76.62 & 79.12 & 79.22 & 79.22 & 78.89 & 66.98 & 54.13 & 52.77 \\
\hline & 4.0 & 53.01 & 54.48 & 79.52 & 82.25 & 82.37 & 82.37 & 82.00 & 68.84 & 54.47 & 52.97 \\
\hline & 5.0 & 53.18 & 54.82 & 82.29 & 85.26 & 85.39 & 85.39 & 84.99 & 70.64 & 54.81 & 53.13 \\
\hline & 6.0 & 53.38 & 55.27 & 85.98 & 89.24 & 89.39 & 89.39 & 88.95 & 73.05 & 55.26 & 53.32 \\
\hline & 7.0 & 53.37 & 53.10 & 55.81 & 56.67 & 56.81 & 56.81 & 56.44 & 54.62 & 53.09 & 53.29 \\
\hline & 7.5 & 53.38 & 52.61 & 52.94 & 53.16 & 53.28 & 53.28 & 53.07 & 52.75 & 52.59 & 53.30 \\
\hline \multirow{9}{*}{ A-2 } & 7.5 & 53.38 & 52.61 & 52.94 & 53.16 & 53.28 & 53.28 & 53.07 & 52.75 & 52.59 & 53.30 \\
\hline & 8.0 & 53.52 & 53.30 & 56.47 & 57.45 & 57.61 & 57.61 & 57.20 & 55.09 & 53.28 & 53.44 \\
\hline & 9.0 & 53.91 & 56.13 & 92.23 & 95.96 & 96.14 & 96.14 & 95.64 & 77.21 & 56.12 & 53.88 \\
\hline & 10.0 & 54.08 & 56.35 & 93.09 & 96.90 & 97.09 & 97.09 & 96.56 & 77.82 & 56.33 & 54.03 \\
\hline & 11.0 & 54.29 & 56.66 & 94.77 & 98.71 & 98.93 & 98.93 & 98.35 & 78.97 & 56.64 & 54.21 \\
\hline & 12.0 & 54.47 & 56.94 & 96.51 & 100.66 & 100.93 & 100.93 & 100.25 & 80.15 & 56.93 & 54.39 \\
\hline & 13.0 & 54.65 & 57.17 & 96.91 & 101.18 & 101.46 & 101.46 & 100.74 & 80.49 & 57.16 & 54.58 \\
\hline & 14.0 & 54.80 & 57.38 & 99.11 & 103.66 & 103.95 & 103.95 & 103.23 & 81.92 & 57.36 & 54.73 \\
\hline & 15.0 & 54.68 & 53.80 & 54.53 & 55.01 & 55.29 & 55.29 & 54.79 & 54.15 & 53.77 & 54.58 \\
\hline \multirow{9}{*}{ A-3 } & 15.0 & 54.68 & 53.80 & 54.53 & 55.01 & 55.29 & 55.29 & 54.79 & 54.15 & 53.77 & 54.58 \\
\hline & 16.0 & 55.14 & 57.45 & 96.21 & 100.38 & 100.69 & 100.69 & 99.94 & 80.16 & 57.43 & 55.02 \\
\hline & 17.0 & 55.31 & 57.61 & 95.93 & 100.09 & 100.42 & 100.42 & 99.63 & 80.05 & 57.60 & 55.24 \\
\hline & 18.0 & 55.47 & 57.78 & 96.09 & 100.31 & 100.65 & 100.65 & 99.83 & 80.22 & 57.77 & 55.42 \\
\hline & 19.0 & 55.65 & 58.02 & 96.70 & 101.03 & 101.40 & 101.40 & 100.54 & 80.69 & 58.00 & 55.58 \\
\hline & 20.0 & 55.81 & 58.24 & 97.42 & 101.86 & 102.24 & 102.24 & 101.35 & 81.21 & 58.22 & 55.70 \\
\hline & 21.0 & 55.98 & 58.52 & 98.51 & 103.11 & 103.51 & 103.51 & 102.59 & 81.99 & 58.49 & 55.88 \\
\hline & 22.0 & 55.89 & 55.76 & 59.86 & 61.36 & 61.79 & 61.79 & 60.88 & 58.08 & 55.74 & 55.80 \\
\hline & 22.5 & 55.87 & 55.10 & 56.05 & 56.72 & 57.14 & 57.14 & 56.41 & 55.58 & 55.08 & 55.81 \\
\hline \multirow{9}{*}{ A-4 } & 22.5 & 55.87 & 55.10 & 56.05 & 56.72 & 57.14 & 57.14 & 56.41 & 55.58 & 55.08 & 55.81 \\
\hline & 23.0 & 56.02 & 55.95 & 60.30 & 61.87 & 62.31 & 62.31 & 61.38 & 58.41 & 55.92 & 55.93 \\
\hline & 24.0 & 56.41 & 59.30 & 102.91 & 107.85 & 108.28 & 108.28 & 107.30 & 84.85 & 59.28 & 56.29 \\
\hline & 25.0 & 56.54 & 59.44 & 102.45 & 107.36 & 107.81 & 107.81 & 106.80 & 84.62 & 59.42 & 56.43 \\
\hline & 26.0 & 56.68 & 59.63 & 102.36 & 107.28 & 107.74 & 107.74 & 106.71 & 84.64 & 59.61 & 56.61 \\
\hline & 27.0 & 56.81 & 59.82 & 102.70 & 107.65 & 108.13 & 108.13 & 107.07 & 84.91 & 59.81 & 56.75 \\
\hline & 28.0 & 56.92 & 60.01 & 102.90 & 107.87 & 108.36 & 108.36 & 107.27 & 85.09 & 59.99 & 56.84 \\
\hline & 29.0 & 57.00 & 60.07 & 103.04 & 107.97 & 108.47 & 108.47 & 107.39 & 85.18 & 60.05 & 56.89 \\
\hline & 30.0 & 56.82 & 56.53 & 57.79 & 58.67 & 59.22 & 59.21 & 58.26 & 57.20 & 56.50 & 56.70 \\
\hline \multirow{9}{*}{ A-5 } & 30.0 & 56.82 & 56.53 & 57.79 & 58.67 & 59.22 & 59.22 & 58.26 & 57.20 & 56.50 & 56.70 \\
\hline & 31.0 & 57.19 & 60.37 & 102.48 & 107.38 & 107.88 & 107.88 & 106.79 & 84.98 & 60.35 & 57.11 \\
\hline & 32.0 & 57.30 & 60.46 & 101.08 & 105.90 & 106.42 & 106.42 & 105.29 & 84.20 & 60.44 & 57.20 \\
\hline & 33.0 & 57.38 & 60.59 & 101.09 & 105.89 & 106.42 & 106.42 & 105.28 & 84.25 & 60.57 & 57.26 \\
\hline & 34.0 & 57.46 & 60.69 & 100.09 & 104.81 & 105.36 & 105.36 & 104.19 & 83.70 & 60.67 & 57.35 \\
\hline & 35.0 & 57.52 & 60.73 & 98.71 & 103.32 & 103.87 & 103.87 & 102.70 & 82.91 & 60.72 & 57.44 \\
\hline & 36.0 & 57.59 & 60.86 & 98.27 & 102.84 & 103.41 & 103.41 & 102.22 & 82.71 & 60.85 & 57.52 \\
\hline & 37.0 & 57.44 & 58.38 & 62.60 & 64.30 & 64.93 & 64.93 & 63.70 & 60.79 & 58.36 & 57.37 \\
\hline & 37.5 & 57.39 & 57.80 & 59.18 & 60.16 & 60.78 & 60.78 & 59.70 & 58.56 & 57.77 & 57.28 \\
\hline \multirow{9}{*}{ A-6 } & $\begin{array}{l}37.5 \\
\end{array}$ & 57.39 & 57.80 & 59.19 & 60.16 & 60.78 & 60.78 & 59.70 & 58.56 & 57.77 & 57.28 \\
\hline & 38.0 & 57.47 & 58.39 & 62.23 & 63.82 & 64.44 & 64.44 & 63.25 & 60.58 & 58.37 & 57.36 \\
\hline & 39.0 & 57.67 & 60.88 & 94.59 & 98.76 & 99.32 & 99.32 & 98.16 & 80.56 & 60.86 & 57.57 \\
\hline & $\begin{array}{l}40.0 \\
\end{array}$ & 57.69 & 60.77 & 91.61 & 95.40 & 95.97 & 95.97 & 94.81 & 78.72 & 60.75 & 57.60 \\
\hline & 41.0 & 57.70 & 60.70 & 89.17 & 92.72 & 93.25 & 93.25 & 92.17 & 77.22 & 60.69 & 57.62 \\
\hline & 42.0 & 57.70 & 60.64 & 86.85 & 90.22 & 90.76 & 90.76 & 89.67 & 75.82 & 60.62 & 57.61 \\
\hline & 43.0 & 57.70 & 60.68 & 86.63 & 89.94 & 90.47 & 90.47 & 89.40 & 75.70 & 60.66 & 57.61 \\
\hline & 44.0 & 57.65 & 60.56 & 84.36 & 87.46 & 87.99 & 87.99 & 86.94 & 74.30 & 60.55 & 57.60 \\
\hline & 45.0 & 57.49 & 58.70 & 60.05 & 61.01 & 61.63 & 61.63 & 60.55 & 59.48 & 58.69 & 57.46 \\
\hline
\end{tabular}


Table B - 18: Temperature $\left({ }^{\circ} \mathrm{C}\right)$ map of the west side of position A at BOC 145A (total 51.7 EFPD).

\begin{tabular}{|c|c|c|c|c|c|c|c|c|c|c|c|}
\hline \multirow{2}{*}{$\begin{array}{c}\text { Plate } \\
\text { Location }\end{array}$} & \multirow{2}{*}{$\begin{array}{c}\text { Axial } \\
\text { Location (in) }\end{array}$} & \multicolumn{10}{|c|}{ Width of Plate (inches) } \\
\hline & & 0.00 & 0.08 & 0.16 & 0.25 & 0.50 & 1.00 & 1.50 & 2.00 & 2.08 & 2.16 \\
\hline \multirow{9}{*}{ A-1 } & 0.0 & 51.67 & 51.77 & 51.76 & 51.76 & 51.77 & 51.77 & 51.76 & 51.76 & 51.77 & 51.67 \\
\hline & 1.0 & 52.61 & 53.64 & 73.27 & 75.45 & 75.52 & 75.52 & 75.26 & 64.80 & 53.64 & 52.57 \\
\hline & 2.0 & 52.79 & 53.96 & 76.16 & 78.58 & 78.66 & 78.66 & 78.36 & 66.63 & 53.96 & 52.72 \\
\hline & 3.0 & 52.96 & 54.16 & 76.67 & 79.18 & 79.29 & 79.29 & 78.95 & 67.02 & 54.16 & 52.87 \\
\hline & 4.0 & 53.18 & 54.52 & 79.61 & 82.37 & 82.49 & 82.49 & 82.11 & 68.91 & 54.52 & 53.10 \\
\hline & 5.0 & 53.37 & 54.88 & 82.42 & 85.41 & 85.56 & 85.56 & 85.13 & 70.74 & 54.87 & 53.28 \\
\hline & 6.0 & 53.60 & 55.36 & 86.15 & 89.45 & 89.62 & 89.62 & 89.14 & 73.18 & 55.35 & 53.47 \\
\hline & 7.0 & 53.56 & 53.22 & 55.99 & 56.88 & 57.05 & 57.05 & 56.64 & 54.78 & 53.21 & 53.42 \\
\hline & 7.5 & 53.56 & 52.73 & 53.14 & 53.40 & 53.55 & 53.55 & 53.28 & 52.92 & 52.72 & 53.41 \\
\hline \multirow{9}{*}{ A-2 } & 7.5 & 53.56 & 52.73 & 53.14 & 53.40 & 53.55 & 53.55 & 53.28 & 52.92 & 52.72 & 53.41 \\
\hline & 8.0 & 53.73 & 53.43 & 56.67 & 57.70 & 57.89 & 57.89 & 57.43 & 55.27 & 53.42 & 53.57 \\
\hline & 9.0 & 54.18 & 56.28 & 92.51 & 96.29 & 96.51 & 96.51 & 95.94 & 77.42 & 56.27 & 54.11 \\
\hline & 10.0 & 54.37 & 56.53 & 93.41 & 97.28 & 97.52 & 97.52 & 96.91 & 78.07 & 56.52 & 54.27 \\
\hline & 11.0 & 54.60 & 56.88 & 95.14 & 99.18 & 99.48 & 99.48 & 98.76 & 79.26 & 56.86 & 54.44 \\
\hline & 12.0 & 54.79 & 57.20 & 96.93 & 101.25 & 101.58 & 101.58 & 100.80 & 80.49 & 57.18 & 54.62 \\
\hline & 13.0 & 54.99 & 57.47 & 97.39 & 101.85 & 102.21 & 102.21 & 101.36 & 80.87 & 57.45 & 54.83 \\
\hline & 14.0 & 55.16 & 57.72 & 99.74 & 104.41 & 104.78 & 104.78 & 103.92 & 82.35 & 57.70 & 54.99 \\
\hline & 15.0 & 54.99 & 54.17 & 55.08 & 55.66 & 56.01 & 56.01 & 55.39 & 54.63 & 54.16 & 54.79 \\
\hline \multirow{9}{*}{ A-3 } & 15.0 & 54.99 & 54.17 & 55.08 & 55.66 & 56.01 & 56.01 & 55.39 & 54.63 & 54.16 & 54.79 \\
\hline & 16.0 & 55.52 & 57.86 & 96.86 & 101.26 & 101.67 & 101.67 & 100.76 & 80.68 & 57.83 & 55.28 \\
\hline & 17.0 & 55.71 & 58.05 & 96.63 & 101.05 & 101.48 & 101.48 & 100.52 & 80.61 & 58.03 & 55.58 \\
\hline & 18.0 & 55.89 & 58.26 & 96.85 & 101.34 & 101.80 & 101.80 & 100.79 & 80.82 & 58.25 & 55.79 \\
\hline & 19.0 & 56.09 & 58.55 & 97.58 & 102.16 & 102.65 & 102.65 & 101.59 & 81.35 & 58.54 & 55.94 \\
\hline & 20.0 & 56.26 & 58.82 & 98.42 & 103.08 & 103.60 & 103.60 & 102.49 & 81.93 & 58.80 & 56.05 \\
\hline & 21.0 & 56.45 & 59.15 & 99.65 & 104.43 & 104.97 & 104.97 & 103.81 & 82.76 & 59.13 & 56.24 \\
\hline & 22.0 & 56.32 & 56.45 & 60.80 & 62.45 & 63.01 & 63.01 & 61.90 & 58.91 & 56.43 & 56.13 \\
\hline & 22.5 & 56.28 & 55.81 & 57.02 & 57.87 & 58.41 & 58.40 & 57.47 & 56.44 & 55.79 & 56.18 \\
\hline \multirow{9}{*}{ A-4 } & 22.5 & 56.28 & 55.81 & 57.02 & 57.87 & 58.41 & 58.41 & 57.47 & 56.44 & 55.79 & $\begin{array}{c}56.18 \\
\end{array}$ \\
\hline & 23.0 & 56.46 & 56.67 & 61.29 & 63.01 & 63.58 & 63.58 & 62.44 & 59.29 & 56.65 & 56.26 \\
\hline & 24.0 & 56.91 & 60.06 & 104.22 & 109.37 & 109.94 & 109.94 & 108.71 & 85.75 & 60.04 & 56.66 \\
\hline & 25.0 & 57.05 & 60.25 & 103.83 & 108.96 & 109.56 & 109.56 & 108.28 & 85.58 & 60.22 & 56.82 \\
\hline & 26.0 & 57.21 & 60.49 & 103.83 & 108.97 & 109.61 & 109.61 & 108.28 & 85.66 & 60.47 & 57.05 \\
\hline & 27.0 & 57.35 & 60.74 & 104.25 & 109.43 & 110.09 & 110.09 & 108.73 & 85.98 & 60.72 & 57.22 \\
\hline & 28.0 & 57.48 & 60.98 & 104.53 & 109.74 & 110.42 & 110.42 & 109.02 & 86.22 & 60.96 & 57.31 \\
\hline & 29.0 & 57.55 & 61.10 & 104.75 & 109.94 & 110.63 & 110.63 & 109.23 & 86.38 & 61.08 & 57.32 \\
\hline & 30.0 & 57.32 & 57.62 & 59.22 & 60.32 & 61.03 & 61.03 & 59.80 & 58.48 & 57.59 & 57.08 \\
\hline \multirow{9}{*}{ A-5 } & 30.0 & 57.32 & 57.62 & 59.22 & 60.32 & 61.03 & 61.03 & 59.80 & 58.48 & 57.59 & 57.08 \\
\hline & 31.0 & 57.76 & 61.49 & 104.31 & 109.48 & 110.17 & 110.17 & 108.75 & 86.27 & 61.47 & 57.56 \\
\hline & 32.0 & 57.87 & 61.62 & 102.99 & 108.07 & 108.79 & 108.79 & 107.32 & 85.54 & 61.60 & 57.66 \\
\hline & $\begin{array}{l}33.0 \\
\end{array}$ & 57.96 & 61.80 & 103.06 & 108.13 & 108.87 & 108.87 & 107.38 & 85.64 & 61.77 & 57.71 \\
\hline & 34.0 & 58.05 & 61.96 & 102.15 & 107.15 & 107.91 & 107.91 & 106.38 & 85.16 & 61.93 & 57.81 \\
\hline & 35.0 & 58.12 & 62.05 & 100.84 & 105.74 & 106.52 & 106.51 & 104.97 & 84.43 & 62.03 & 57.94 \\
\hline & 36.0 & 58.19 & 62.24 & 100.49 & 105.36 & 106.14 & 106.14 & 104.58 & 84.30 & 62.22 & 58.04 \\
\hline & 37.0 & 58.00 & 59.81 & 64.39 & 66.33 & 67.15 & 67.15 & 65.61 & 62.43 & 59.79 & 57.85 \\
\hline & 37.5 & 57.93 & 59.25 & 61.01 & 62.24 & 63.05 & 63.05 & 61.65 & 60.23 & 59.22 & 57.71 \\
\hline \multirow{9}{*}{ A-6 } & 37.5 & 57.93 & 59.25 & 61.01 & 62.24 & 63.05 & 63.05 & 61.65 & 60.23 & 59.22 & 57.71 \\
\hline & 38.0 & 58.03 & 59.86 & 64.05 & 65.89 & 66.69 & 66.69 & 65.19 & 62.26 & 59.83 & 57.81 \\
\hline & 39.0 & 58.27 & 62.38 & 96.95 & 101.46 & 102.24 & 102.24 & 100.70 & 82.29 & 62.35 & 58.06 \\
\hline & 40.0 & 58.29 & 62.31 & 93.94 & 98.17 & 98.97 & 98.97 & 97.43 & 80.50 & 62.28 & 58.09 \\
\hline & 41.0 & 58.30 & 62.29 & 91.51 & 95.52 & 96.32 & 96.32 & 94.79 & 79.08 & 62.26 & 58.11 \\
\hline & 42.0 & 58.30 & 62.28 & 89.23 & 93.03 & 93.83 & 93.83 & 92.29 & 77.73 & 62.25 & 58.08 \\
\hline & 43.0 & 58.29 & 62.35 & 89.06 & 92.80 & 93.60 & 93.60 & 92.07 & 77.66 & 62.32 & 58.10 \\
\hline & 44.0 & 58.24 & 62.28 & 86.88 & 90.34 & 91.13 & 91.13 & 89.62 & 76.32 & 62.25 & 58.12 \\
\hline & 45.0 & 58.04 & 60.45 & 62.17 & 63.38 & 64.20 & 64.19 & 62.79 & 61.43 & 60.42 & 57.96 \\
\hline
\end{tabular}


Table B - 19: Temperature $\left({ }^{\circ} \mathrm{C}\right)$ map of the east side of position B at BOC 145A (total 51.7 EFPD).

\begin{tabular}{|c|c|c|c|c|c|c|c|c|c|c|c|}
\hline \multirow{2}{*}{$\begin{array}{c}\text { Plate } \\
\text { Location }\end{array}$} & \multirow{2}{*}{$\begin{array}{c}\text { Axial } \\
\text { Location (in) }\end{array}$} & \multicolumn{10}{|c|}{ Width of Plate (inches) } \\
\hline & & 0.00 & 0.08 & 0.16 & 0.25 & 0.50 & 1.00 & 1.50 & 2.00 & 2.08 & 2.16 \\
\hline \multirow{9}{*}{ B-1 } & 0.0 & 51.67 & 51.79 & 51.76 & 51.76 & 51.77 & 51.77 & 51.76 & 51.76 & 51.79 & 51.67 \\
\hline & 1.0 & 52.95 & 53.72 & 73.30 & 75.48 & 75.54 & 75.54 & 75.48 & 73.30 & 53.69 & 52.85 \\
\hline & 2.0 & 53.18 & 54.05 & 76.24 & 78.66 & 78.75 & 78.75 & 78.66 & 76.23 & 54.00 & 52.92 \\
\hline & 3.0 & 53.39 & 54.26 & 76.73 & 79.25 & 79.35 & 79.35 & 79.25 & 76.73 & 54.20 & 53.02 \\
\hline & 4.0 & 53.65 & 54.61 & 79.35 & 82.08 & 82.21 & 82.21 & 82.08 & 79.35 & 54.55 & 53.30 \\
\hline & 5.0 & 53.88 & 55.00 & 82.46 & 85.45 & 85.60 & 85.60 & 85.45 & 82.46 & 54.93 & 53.47 \\
\hline & 6.0 & 54.15 & 55.48 & 86.10 & 89.38 & 89.56 & 89.56 & 89.38 & 86.10 & 55.40 & 53.66 \\
\hline & 7.0 & 54.14 & 53.36 & 56.00 & 56.89 & 57.06 & 57.06 & 56.89 & 56.00 & 53.27 & 53.62 \\
\hline & 7.5 & 54.16 & 52.89 & 53.16 & 53.41 & 53.56 & 53.56 & 53.41 & 53.16 & 52.79 & 53.61 \\
\hline \multirow{9}{*}{ B-2 } & 7.5 & 54.16 & 52.89 & 53.16 & 53.41 & 53.56 & 53.56 & 53.41 & 53.16 & 52.79 & 53.61 \\
\hline & 8.0 & 54.35 & 53.54 & 56.46 & 57.43 & 57.61 & 57.61 & 57.43 & 56.45 & 53.44 & 53.77 \\
\hline & 9.0 & 54.83 & 56.23 & 90.13 & 93.74 & 93.95 & 93.95 & 93.74 & 90.13 & 56.19 & 54.73 \\
\hline & 10.0 & 55.05 & 56.51 & 91.37 & 95.09 & 95.32 & 95.32 & 95.09 & 91.37 & 56.44 & 54.80 \\
\hline & 11.0 & 55.31 & 56.88 & 93.25 & 97.11 & 97.37 & 97.37 & 97.11 & 93.24 & 56.77 & 54.81 \\
\hline & 12.0 & 55.54 & 57.17 & 94.53 & 98.51 & 98.83 & 98.83 & 98.51 & 94.52 & 57.05 & 54.85 \\
\hline & 13.0 & 55.76 & 57.44 & 94.98 & 99.08 & 99.43 & 99.43 & 99.08 & 94.97 & 57.33 & 55.12 \\
\hline & 14.0 & 55.96 & 57.73 & 97.58 & 102.02 & 102.39 & 102.39 & 102.02 & 97.58 & 57.62 & 55.26 \\
\hline & 15.0 & 55.81 & 54.40 & 55.11 & 55.67 & 56.02 & 56.02 & 55.67 & 55.10 & 54.26 & 55.09 \\
\hline \multirow{9}{*}{ B-3 } & 15.0 & 55.81 & 54.40 & 55.11 & 55.67 & 56.02 & 56.02 & 55.67 & 55.10 & 54.26 & 55.09 \\
\hline & 16.0 & 56.39 & 58.38 & 100.24 & 104.99 & 105.40 & 105.40 & 104.99 & 100.24 & 58.24 & 55.57 \\
\hline & $\mathbf{1 7 . 0}$ & 56.60 & 58.62 & 100.55 & 105.34 & 105.78 & 105.78 & 105.34 & 100.55 & 58.53 & 56.27 \\
\hline & 18.0 & 56.79 & 58.85 & 100.83 & 105.66 & 106.13 & 106.13 & 105.66 & 100.83 & 58.80 & 56.62 \\
\hline & 19.0 & 57.01 & 59.12 & 101.23 & 106.11 & 106.61 & 106.61 & 106.11 & 101.23 & 59.03 & 56.65 \\
\hline & 20.0 & 57.20 & 59.41 & 102.20 & 107.16 & 107.68 & 107.68 & 107.16 & 102.19 & 59.27 & 56.53 \\
\hline & 21.0 & 57.40 & 59.74 & 103.28 & 108.34 & 108.88 & 108.88 & 108.34 & 103.27 & 59.59 & 56.64 \\
\hline & 22.0 & 57.27 & 56.81 & 61.29 & 63.00 & 63.56 & 63.56 & 63.00 & 61.27 & 56.67 & 56.47 \\
\hline & 22.5 & 57.23 & 56.11 & 57.18 & 58.02 & 58.56 & 58.56 & 58.02 & 57.17 & 56.05 & 57.11 \\
\hline \multirow{9}{*}{ B-4 } & 22.5 & 57.23 & 56.11 & 57.18 & 58.02 & 58.56 & 58.56 & 58.02 & 57.17 & 56.05 & 57.11 \\
\hline & 23.0 & 57.41 & 56.97 & 61.42 & 63.14 & 63.70 & 63.70 & 63.13 & 61.41 & 56.83 & 56.61 \\
\hline & 24.0 & 57.87 & 60.34 & 104.21 & 109.33 & 109.91 & 109.91 & 109.33 & 104.20 & 60.19 & 56.96 \\
\hline & 25.0 & 58.01 & 60.50 & 103.37 & 108.45 & 109.05 & 109.05 & 108.45 & 103.36 & 60.34 & 57.30 \\
\hline & 26.0 & 58.16 & 60.79 & 103.90 & 109.03 & 109.66 & 109.66 & 109.03 & 103.89 & 60.69 & 57.82 \\
\hline & 27.0 & 58.30 & 61.02 & 104.00 & 109.15 & 109.81 & 109.81 & 109.15 & 103.99 & 60.96 & 58.10 \\
\hline & 28.0 & 58.42 & 61.26 & 104.27 & 109.45 & 110.12 & 110.12 & 109.45 & 104.26 & 61.15 & 58.04 \\
\hline & 29.0 & 58.49 & 61.40 & 104.66 & 109.82 & 110.50 & 110.50 & 109.82 & 104.65 & 61.25 & 57.79 \\
\hline & 30.0 & 58.24 & 57.95 & 59.38 & 60.47 & 61.18 & 61.17 & 60.46 & 59.36 & 57.78 & 57.41 \\
\hline \multirow{9}{*}{ B-5 } & 30.0 & 58.24 & 57.95 & 59.38 & 60.47 & 61.18 & 61.17 & 60.46 & 59.36 & 57.78 & 57.41 \\
\hline & 31.0 & 58.67 & 61.57 & 101.69 & 106.62 & 107.32 & 107.32 & 106.62 & 101.68 & 61.44 & 57.97 \\
\hline & 32.0 & 58.76 & 61.69 & 100.17 & 105.00 & 105.72 & 105.72 & 105.00 & 100.16 & 61.54 & 57.98 \\
\hline & 33.0 & 58.83 & 61.87 & 100.27 & 105.09 & 105.83 & 105.83 & 105.09 & 100.25 & 61.72 & 57.96 \\
\hline & 34.0 & 58.90 & 62.08 & 99.95 & 104.75 & 105.51 & 105.50 & 104.74 & 99.94 & 61.93 & 58.20 \\
\hline & 35.0 & 58.95 & 62.15 & 98.48 & 103.15 & 103.92 & 103.92 & 103.15 & 98.46 & 62.04 & 58.57 \\
\hline & 36.0 & 58.99 & 62.32 & 97.88 & 102.50 & 103.29 & 103.29 & 102.50 & 97.87 & 62.25 & 58.71 \\
\hline & 37.0 & 58.78 & 60.08 & 64.29 & 66.16 & 66.96 & 66.96 & 66.15 & 64.27 & 59.97 & 58.49 \\
\hline & 37.5 & 58.70 & 59.56 & 61.17 & 62.37 & 63.17 & 63.17 & 62.36 & 61.15 & 59.42 & 57.96 \\
\hline \multirow{9}{*}{ B-6 } & 37.5 & 58.70 & 59.56 & 61.17 & 62.37 & 63.17 & 63.17 & 62.36 & 61.15 & 59.42 & 57.96 \\
\hline & 38.0 & 58.78 & 60.15 & 64.11 & 65.91 & 66.70 & 66.70 & 65.90 & 64.09 & 60.01 & 58.15 \\
\hline & 39.0 & 59.00 & 62.59 & 96.01 & 100.42 & 101.20 & 101.19 & 100.41 & 96.00 & 62.45 & 58.30 \\
\hline & 40.0 & 58.98 & 62.54 & 93.37 & 97.54 & 98.33 & 98.33 & 97.53 & 93.36 & 62.42 & 58.30 \\
\hline & 41.0 & 58.96 & 62.53 & 91.05 & 95.01 & 95.80 & 95.80 & 95.00 & 91.04 & 62.41 & 58.34 \\
\hline & 42.0 & 58.92 & 62.50 & 88.71 & 92.43 & 93.22 & 93.22 & 92.42 & 88.70 & 62.37 & 58.23 \\
\hline & 43.0 & 58.88 & 62.58 & 88.54 & 92.20 & 93.00 & 92.99 & 92.19 & 88.53 & 62.45 & 58.35 \\
\hline & 44.0 & 58.79 & 62.50 & 86.44 & 89.82 & 90.61 & 90.61 & 89.81 & 86.42 & 62.42 & 58.54 \\
\hline & 45.0 & 58.57 & 60.72 & 62.36 & 63.56 & 64.35 & 64.35 & 63.54 & 62.34 & 60.67 & 58.45 \\
\hline
\end{tabular}


Table B - 20: Temperature $\left({ }^{\circ} \mathrm{C}\right)$ map of the west side of position B at BOC 145A (total 51.7 EFPD).

\begin{tabular}{|c|c|c|c|c|c|c|c|c|c|c|c|}
\hline \multirow{2}{*}{$\begin{array}{c}\text { Plate } \\
\text { Location }\end{array}$} & \multirow{2}{*}{$\begin{array}{c}\text { Axial } \\
\text { Location (in) }\end{array}$} & \multicolumn{10}{|c|}{ Width of Plate (inches) } \\
\hline & & 0.00 & 0.08 & 0.16 & 0.25 & 0.50 & 1.00 & 1.50 & 2.00 & 2.08 & 2.16 \\
\hline \multirow{9}{*}{ B-1 } & 0.0 & 51.67 & 51.79 & 51.76 & 51.76 & 51.77 & 51.77 & 51.76 & 51.76 & 51.79 & 51.67 \\
\hline & 1.0 & 53.27 & 53.69 & 73.29 & 75.47 & 75.53 & 75.53 & 75.47 & 73.29 & 53.68 & 53.15 \\
\hline & 2.0 & 53.53 & 54.02 & 76.21 & 78.63 & 78.71 & 78.71 & 78.63 & 76.21 & 53.98 & 53.09 \\
\hline & 3.0 & 53.77 & 54.21 & 76.69 & 79.19 & 79.28 & 79.28 & 79.19 & 76.68 & 54.17 & 53.05 \\
\hline & 4.0 & 54.06 & 54.54 & 79.27 & 81.98 & 82.09 & 82.09 & 81.98 & 79.27 & 54.50 & 53.37 \\
\hline & 5.0 & 54.32 & 54.91 & 82.34 & 85.31 & 85.43 & 85.43 & 85.31 & 82.34 & 54.87 & 53.49 \\
\hline & 6.0 & 54.62 & 55.37 & 85.95 & 89.19 & 89.34 & 89.34 & 89.19 & 85.94 & 55.31 & 53.69 \\
\hline & 7.0 & 54.67 & 53.22 & 55.85 & 56.70 & 56.84 & 56.84 & 56.69 & 55.84 & 53.16 & 53.75 \\
\hline & 7.5 & 54.72 & 52.74 & 52.99 & 53.20 & 53.31 & 53.31 & 53.20 & 52.98 & 52.67 & 53.76 \\
\hline \multirow{9}{*}{ B-2 } & 7.5 & 54.72 & 52.74 & 52.99 & 53.20 & 53.31 & 53.31 & 53.20 & 52.98 & 52.67 & 53.76 \\
\hline & 8.0 & 54.90 & 53.39 & 56.28 & 57.21 & 57.36 & 57.36 & 57.20 & 56.27 & 53.32 & 53.89 \\
\hline & 9.0 & 55.37 & 56.06 & 89.89 & 93.44 & 93.61 & 93.60 & 93.43 & 89.89 & 56.03 & 55.40 \\
\hline & 10.0 & 55.61 & 56.32 & 91.09 & 94.74 & 94.92 & 94.92 & 94.74 & 91.09 & 56.27 & 55.34 \\
\hline & 11.0 & 55.89 & 56.66 & 92.91 & 96.70 & 96.90 & 96.90 & 96.69 & 92.91 & 56.58 & 55.04 \\
\hline & 12.0 & 56.13 & 56.91 & 94.14 & 98.02 & 98.24 & 98.24 & 98.02 & 94.13 & 56.82 & 54.82 \\
\hline & 13.0 & 56.37 & 57.15 & 94.54 & 98.48 & 98.73 & 98.73 & 98.48 & 94.53 & 57.07 & 55.12 \\
\hline & 14.0 & 56.57 & 57.40 & 97.09 & 101.32 & 101.60 & 101.60 & 101.32 & 97.08 & 57.32 & 55.22 \\
\hline & 15.0 & 56.50 & 54.02 & 54.62 & 55.07 & 55.33 & 55.33 & 55.06 & 54.60 & 53.93 & 55.20 \\
\hline \multirow{9}{*}{ B-3 } & 15.0 & 56.50 & 54.02 & 54.62 & 55.07 & 55.33 & 55.33 & 55.06 & 54.60 & 53.93 & 55.20 \\
\hline & 16.0 & 57.05 & 57.99 & 99.58 & 104.19 & 104.48 & 104.48 & 104.18 & 99.57 & 57.88 & 55.62 \\
\hline & 17.0 & 57.25 & 58.21 & 99.84 & 104.48 & 104.81 & 104.81 & 104.48 & 99.83 & 58.14 & 56.88 \\
\hline & 18.0 & 57.45 & 58.41 & 100.07 & 104.74 & 105.08 & 105.08 & 104.73 & 100.06 & 58.36 & 57.42 \\
\hline & 19.0 & 57.67 & 58.64 & 100.40 & 105.11 & 105.47 & 105.47 & 105.10 & 100.39 & 58.56 & 57.23 \\
\hline & 20.0 & 57.85 & 58.89 & 101.31 & 106.09 & 106.47 & 106.47 & 106.08 & 101.29 & 58.78 & 56.73 \\
\hline & 21.0 & 58.04 & 59.17 & 102.32 & 107.18 & 107.58 & 107.58 & 107.18 & 102.30 & 59.06 & 56.66 \\
\hline & 22.0 & 57.95 & 56.20 & 60.49 & 62.04 & 62.47 & 62.47 & 62.03 & 60.47 & 56.09 & 56.40 \\
\hline & 22.5 & 57.93 & 55.48 & 56.36 & 57.02 & 57.42 & 57.42 & 57.01 & 56.34 & 55.43 & 58.05 \\
\hline \multirow{9}{*}{ B-4 } & 22.5 & 57.93 & 55.48 & 56.36 & 57.02 & 57.42 & 57.42 & 57.01 & 56.34 & 55.43 & 58.05 \\
\hline & 23.0 & 58.09 & 56.34 & 60.59 & 62.14 & 62.57 & 62.57 & 62.13 & 60.57 & 56.23 & 56.53 \\
\hline & 24.0 & 58.49 & 59.69 & 103.11 & 108.02 & 108.43 & 108.43 & 108.01 & 103.09 & 59.56 & 56.80 \\
\hline & 25.0 & 58.61 & 59.81 & 102.21 & 107.06 & 107.50 & 107.50 & 107.06 & 102.19 & 59.68 & 57.43 \\
\hline & 26.0 & 58.75 & 60.06 & 102.67 & 107.56 & 108.01 & 108.01 & 107.55 & 102.65 & 59.96 & 58.41 \\
\hline & 27.0 & 58.87 & 60.24 & 102.70 & 107.60 & 108.06 & 108.06 & 107.59 & 102.68 & 60.17 & 58.82 \\
\hline & 28.0 & 58.97 & 60.43 & 102.90 & 107.81 & 108.29 & 108.29 & 107.80 & 102.87 & 60.34 & 58.53 \\
\hline & 29.0 & 59.01 & 60.53 & 103.21 & 108.10 & 108.58 & 108.57 & 108.09 & 103.18 & 60.39 & 57.83 \\
\hline & 30.0 & 58.80 & 57.02 & 58.17 & 59.02 & 59.55 & 59.55 & 59.01 & 58.14 & 56.88 & 57.29 \\
\hline \multirow{9}{*}{ B-5 } & 30.0 & 58.80 & 57.02 & 58.17 & 59.02 & 59.55 & 59.55 & 59.01 & 58.14 & 56.88 & 57.29 \\
\hline & 31.0 & 59.15 & 60.63 & 100.12 & 104.77 & 105.26 & 105.25 & 104.76 & 100.10 & 60.51 & 57.84 \\
\hline & 32.0 & 59.21 & 60.71 & 98.54 & 103.08 & 103.58 & 103.58 & 103.07 & 98.51 & 60.58 & 57.69 \\
\hline & 33.0 & 59.25 & 60.85 & 98.58 & 103.10 & 103.62 & 103.61 & 103.09 & 98.55 & 60.71 & 57.62 \\
\hline & 34.0 & 59.28 & 61.01 & 98.18 & 102.67 & 103.20 & 103.19 & 102.66 & 98.15 & 60.87 & 58.11 \\
\hline & 35.0 & 59.29 & 61.04 & 96.63 & 100.99 & 101.52 & 101.52 & 100.97 & 96.60 & 60.93 & 58.86 \\
\hline & 36.0 & 59.30 & 61.15 & 95.95 & 100.25 & 100.79 & 100.79 & 100.23 & 95.92 & 61.06 & 59.02 \\
\hline & 37.0 & 59.09 & 58.86 & 62.74 & 64.34 & 64.95 & 64.94 & 64.33 & 62.71 & 58.75 & 58.85 \\
\hline & 37.5 & 59.01 & 58.33 & 59.59 & 60.52 & 61.10 & 61.10 & 60.50 & 59.55 & 58.19 & 57.67 \\
\hline \multirow{9}{*}{ B-6 } & 37.5 & 59.01 & 58.33 & 59.59 & 60.52 & 61.10 & 61.10 & 60.50 & 59.55 & 58.19 & 57.67 \\
\hline & 38.0 & 59.05 & 58.90 & 62.54 & 64.07 & 64.66 & 64.65 & 64.05 & 62.50 & 58.77 & 58.00 \\
\hline & 39.0 & 59.19 & 61.32 & 93.97 & 98.00 & 98.54 & 98.53 & 97.99 & 93.94 & 61.19 & 57.95 \\
\hline & 40.0 & 59.14 & 61.24 & 91.36 & 95.06 & 95.60 & 95.59 & 95.04 & 91.32 & 61.11 & 57.82 \\
\hline & 41.0 & 59.07 & 61.19 & 89.03 & 92.49 & 92.99 & 92.98 & 92.47 & 88.99 & 61.06 & 57.94 \\
\hline & 42.0 & 58.99 & 61.12 & 86.64 & 89.91 & 90.42 & 90.41 & 89.89 & 86.60 & 60.99 & 57.72 \\
\hline & 43.0 & 58.92 & 61.16 & 86.42 & 89.63 & 90.14 & 90.13 & 89.62 & 86.38 & 61.03 & 58.02 \\
\hline & 44.0 & 58.78 & 61.05 & 84.23 & 87.24 & 87.74 & 87.73 & 87.22 & 84.20 & 60.95 & 58.44 \\
\hline & 45.0 & 58.57 & 59.24 & 60.51 & 61.43 & 62.01 & 62.01 & 61.40 & 60.47 & 59.16 & 58.43 \\
\hline
\end{tabular}


Table B - 21: Temperature $\left({ }^{\circ} \mathrm{C}\right)$ map of the east side of position A at MOC1 145A (total 69.7 EFPD).

\begin{tabular}{|c|c|c|c|c|c|c|c|c|c|c|c|}
\hline \multirow{2}{*}{$\begin{array}{c}\text { Plate } \\
\text { Location }\end{array}$} & \multirow{2}{*}{$\begin{array}{c}\text { Axial } \\
\text { Location (in) }\end{array}$} & \multicolumn{10}{|c|}{ Width of Plate (inches) } \\
\hline & & 0.00 & 0.08 & 0.16 & 0.25 & 0.50 & 1.00 & 1.50 & 2.00 & 2.08 & 2.16 \\
\hline \multirow{9}{*}{ A-1 } & 0.0 & 51.67 & 51.77 & 51.76 & 51.77 & 51.77 & 51.77 & 51.77 & 51.76 & 51.77 & 51.67 \\
\hline & 1.0 & 52.51 & 53.83 & 75.34 & 77.69 & 77.76 & 77.76 & 77.69 & 75.34 & 53.82 & 52.49 \\
\hline & 2.0 & 52.67 & 54.12 & 77.87 & 80.43 & 80.52 & 80.52 & 80.43 & 77.87 & 54.11 & 52.63 \\
\hline & 3.0 & 52.83 & 54.31 & 78.34 & 80.98 & 81.09 & 81.09 & 80.98 & 78.34 & 54.30 & 52.79 \\
\hline & 4.0 & 53.02 & 54.65 & 81.23 & 84.11 & 84.23 & 84.23 & 84.11 & 81.23 & 54.64 & 52.99 \\
\hline & 5.0 & 53.20 & 54.97 & 83.76 & 86.84 & 86.97 & 86.97 & 86.84 & 83.76 & 54.96 & 53.16 \\
\hline & 6.0 & 53.40 & 55.37 & 86.81 & 90.13 & 90.29 & 90.29 & 90.13 & 86.81 & 55.35 & 53.34 \\
\hline & 7.0 & 53.38 & 53.15 & 55.94 & 56.82 & 56.97 & 56.97 & 56.82 & 55.94 & 53.13 & 53.31 \\
\hline & 7.5 & 53.39 & 52.64 & 53.00 & 53.23 & 53.35 & 53.35 & 53.23 & 53.00 & 52.62 & 53.31 \\
\hline \multirow{9}{*}{ A-2 } & 7.5 & 53.39 & 52.64 & 53.00 & 53.23 & 53.35 & 53.35 & 53.23 & 53.00 & 52.62 & 53.31 \\
\hline & 8.0 & 53.54 & 53.34 & 56.56 & 57.57 & 57.73 & 57.73 & 57.57 & 56.56 & 53.32 & 53.45 \\
\hline & 9.0 & 53.93 & 56.20 & 92.69 & 96.46 & 96.64 & 96.64 & 96.46 & 92.69 & 56.19 & 53.90 \\
\hline & 10.0 & 54.10 & 56.40 & 93.34 & 97.17 & 97.36 & 97.36 & 97.17 & 93.34 & 56.39 & 54.05 \\
\hline & 11.0 & 54.31 & 56.70 & 94.89 & 98.85 & 99.07 & 99.07 & 98.85 & 94.89 & 56.69 & 54.23 \\
\hline & 12.0 & 54.48 & 56.93 & 95.98 & 100.07 & 100.34 & 100.34 & 100.07 & 95.98 & 56.92 & 54.40 \\
\hline & 13.0 & 54.66 & 57.13 & 96.27 & 100.45 & 100.74 & 100.74 & 100.45 & 96.27 & 57.12 & 54.59 \\
\hline & 14.0 & 54.81 & 57.27 & 97.53 & 101.85 & 102.15 & 102.15 & 101.85 & 97.53 & 57.25 & 54.73 \\
\hline & 15.0 & 54.70 & 53.83 & 54.56 & 55.04 & 55.33 & 55.33 & 55.04 & 54.56 & 53.80 & 54.60 \\
\hline \multirow{9}{*}{ A-3 } & 15.0 & 54.70 & 53.83 & 54.56 & 55.04 & 55.33 & 55.33 & 55.04 & 54.56 & 53.80 & 54.60 \\
\hline & 16.0 & 55.13 & 57.27 & 93.87 & 97.77 & 98.04 & 98.04 & 97.77 & 93.86 & 57.24 & 55.01 \\
\hline & 17.0 & 55.30 & 57.43 & 93.74 & 97.64 & 97.93 & 97.93 & 97.64 & 93.73 & 57.41 & 55.24 \\
\hline & 18.0 & 55.46 & 57.60 & 93.89 & 97.82 & 98.14 & 98.14 & 97.82 & 93.89 & 57.58 & 55.41 \\
\hline & 19.0 & 55.64 & 57.81 & 94.37 & 98.35 & 98.72 & 98.72 & 98.35 & 94.36 & 57.80 & 55.57 \\
\hline & 20.0 & 55.79 & 57.98 & 94.53 & 98.56 & 98.94 & 98.94 & 98.56 & 94.53 & 57.96 & 55.68 \\
\hline & 21.0 & 55.96 & 58.23 & 95.43 & 99.60 & 99.99 & 99.99 & 99.60 & 95.43 & 58.21 & 55.85 \\
\hline & 22.0 & 55.88 & 55.68 & 59.49 & 60.91 & 61.34 & 61.34 & 60.91 & 59.49 & 55.66 & 55.79 \\
\hline & 22.5 & 55.86 & 55.07 & 55.97 & 56.63 & 57.03 & 57.03 & 56.63 & 55.97 & 55.05 & 55.80 \\
\hline \multirow{9}{*}{ A-4 } & 22.5 & 55.86 & 55.07 & 55.97 & 56.63 & 57.03 & 57.03 & 56.63 & 55.97 & 55.05 & 55.80 \\
\hline & 23.0 & 56.01 & 55.86 & 59.91 & 61.39 & 61.82 & 61.82 & 61.39 & 59.91 & 55.84 & 55.91 \\
\hline & 24.0 & 56.37 & 58.97 & 99.44 & 104.09 & 104.51 & 104.51 & 104.09 & 99.44 & 58.95 & 56.25 \\
\hline & 25.0 & 56.50 & 59.13 & 99.32 & 103.97 & 104.40 & 104.40 & 103.97 & 99.32 & 59.11 & 56.39 \\
\hline & 26.0 & 56.64 & 59.34 & 99.54 & 104.21 & 104.66 & 104.66 & 104.21 & 99.54 & 59.32 & 56.56 \\
\hline & 27.0 & 56.75 & 59.50 & 99.57 & 104.26 & 104.72 & 104.72 & 104.26 & 99.57 & 59.48 & 56.70 \\
\hline & 28.0 & 56.87 & 59.67 & 99.74 & 104.44 & 104.92 & 104.92 & 104.44 & 99.74 & 59.65 & 56.79 \\
\hline & 29.0 & 56.94 & 59.73 & 99.91 & 104.58 & 105.05 & 105.05 & 104.58 & 99.91 & 59.71 & 56.83 \\
\hline & 30.0 & 56.77 & 56.42 & 57.60 & 58.44 & 58.96 & 58.96 & 58.44 & 57.60 & 56.39 & 56.66 \\
\hline \multirow{9}{*}{ A-5 } & 30.0 & 56.77 & 56.42 & 57.60 & 58.44 & 58.96 & 58.96 & 58.44 & 57.60 & 56.39 & 56.66 \\
\hline & 31.0 & 57.13 & 60.09 & 100.22 & 104.93 & 105.41 & 105.41 & 104.93 & 100.22 & 60.07 & 57.04 \\
\hline & 32.0 & 57.23 & 60.17 & 98.89 & 103.50 & 104.00 & 104.00 & 103.50 & 98.89 & 60.15 & 57.14 \\
\hline & 33.0 & 57.31 & 60.31 & 98.95 & 103.56 & 104.07 & 104.07 & 103.56 & 98.94 & 60.29 & 57.20 \\
\hline & 34.0 & 57.39 & 60.47 & 98.75 & 103.35 & 103.88 & 103.88 & 103.35 & 98.75 & 60.44 & 57.28 \\
\hline & 35.0 & 57.45 & 60.53 & 97.73 & 102.25 & 102.78 & 102.78 & 102.25 & 97.73 & 60.51 & 57.37 \\
\hline & 36.0 & 57.52 & 60.71 & 98.00 & 102.53 & 103.08 & 103.08 & 102.53 & 98.00 & 60.70 & 57.45 \\
\hline & 37.0 & 57.37 & 58.23 & 62.38 & 64.04 & 64.65 & 64.65 & 64.04 & 62.38 & 58.21 & 57.30 \\
\hline & 37.5 & 57.31 & 57.64 & 58.97 & 59.90 & 60.50 & 60.50 & 59.90 & 58.97 & 57.62 & 57.21 \\
\hline \multirow{9}{*}{ A-6 } & 37.5 & 57.31 & 57.64 & 58.97 & 59.90 & 60.50 & 60.50 & 59.90 & 58.97 & 57.62 & 57.21 \\
\hline & 38.0 & 57.39 & 58.24 & 62.04 & 63.61 & 64.20 & 64.20 & 63.61 & 62.04 & 58.22 & 57.29 \\
\hline & 39.0 & 57.60 & 60.75 & 94.70 & 98.86 & 99.40 & 99.40 & 98.86 & 94.70 & 60.73 & 57.50 \\
\hline & 40.0 & 57.61 & 60.65 & 91.83 & 95.63 & 96.18 & 96.18 & 95.63 & 91.83 & 60.63 & 57.53 \\
\hline & 41.0 & 57.62 & 60.62 & 89.89 & 93.49 & 94.00 & 94.00 & 93.49 & 89.89 & 60.61 & 57.54 \\
\hline & 42.0 & 57.63 & 60.57 & 87.71 & 91.14 & 91.66 & 91.66 & 91.14 & 87.71 & 60.55 & 57.53 \\
\hline & 43.0 & 57.62 & 60.61 & 87.50 & 90.88 & 91.40 & 91.40 & 90.88 & 87.50 & 60.60 & 57.54 \\
\hline & 44.0 & 57.58 & 60.49 & 85.32 & 88.49 & 89.00 & 89.00 & 88.49 & 85.32 & 60.49 & 57.53 \\
\hline & 45.0 & 57.41 & 58.54 & 59.87 & 60.80 & 61.41 & 61.41 & 60.80 & 59.87 & 58.53 & 57.38 \\
\hline
\end{tabular}


Table B - 22: Temperature $\left({ }^{\circ} \mathrm{C}\right)$ map of the west side of position A at MOC1 145A (total 69.7 EFPD).

\begin{tabular}{|c|c|c|c|c|c|c|c|c|c|c|c|}
\hline \multirow{2}{*}{$\begin{array}{c}\text { Plate } \\
\text { Location }\end{array}$} & \multirow{2}{*}{$\begin{array}{c}\text { Axial } \\
\text { Location (in) }\end{array}$} & \multicolumn{10}{|c|}{ Width of Plate (inches) } \\
\hline & & 0.00 & 0.08 & 0.16 & 0.25 & 0.50 & 1.00 & 1.50 & 2.00 & 2.08 & 2.16 \\
\hline \multirow{9}{*}{ A-1 } & 0.0 & 51.67 & 51.77 & 51.76 & 51.77 & 51.77 & 51.77 & 51.77 & 51.76 & 51.77 & 51.67 \\
\hline & 1.0 & 52.63 & 53.83 & 75.35 & 77.71 & 77.78 & 77.78 & 77.71 & 75.35 & 53.82 & 52.59 \\
\hline & 2.0 & 52.81 & 54.13 & 77.90 & 80.47 & 80.56 & 80.56 & 80.47 & 77.90 & 54.12 & 52.74 \\
\hline & 3.0 & 52.99 & 54.33 & 78.40 & 81.06 & 81.17 & 81.17 & 81.06 & 78.40 & 54.32 & 52.89 \\
\hline & 4.0 & 53.20 & 54.70 & 81.33 & 84.23 & 84.37 & 84.37 & 84.23 & 81.33 & 54.69 & 53.12 \\
\hline & 5.0 & 53.39 & 55.04 & 83.90 & 87.01 & 87.16 & 87.16 & 87.01 & 83.89 & 55.03 & 53.30 \\
\hline & 6.0 & 53.62 & 55.46 & 86.99 & 90.36 & 90.54 & 90.54 & 90.36 & 86.99 & 55.45 & 53.50 \\
\hline & 7.0 & 53.58 & 53.27 & 56.13 & 57.06 & 57.24 & 57.24 & 57.06 & 56.13 & 53.26 & 53.43 \\
\hline & 7.5 & 53.57 & 52.77 & 53.21 & 53.48 & 53.64 & 53.64 & 53.48 & 53.21 & 52.76 & 53.42 \\
\hline \multirow{9}{*}{ A-2 } & 7.5 & 53.57 & 52.77 & 53.21 & 53.48 & 53.64 & 53.64 & 53.48 & 53.21 & 52.76 & 53.42 \\
\hline & 8.0 & 53.75 & 53.48 & 56.78 & 57.83 & 58.03 & 58.03 & 57.83 & 56.78 & 53.47 & 53.59 \\
\hline & 9.0 & 54.20 & 56.36 & 92.98 & 96.81 & 97.03 & 97.03 & 96.81 & 92.98 & 56.35 & 54.13 \\
\hline & 10.0 & 54.39 & 56.59 & 93.68 & 97.57 & 97.81 & 97.81 & 97.57 & 93.68 & 56.58 & 54.29 \\
\hline & 11.0 & 54.61 & 56.93 & 95.28 & 99.36 & 99.66 & 99.66 & 99.36 & 95.28 & 56.92 & 54.46 \\
\hline & 12.0 & 54.81 & 57.20 & 96.42 & 100.69 & 101.02 & 101.02 & 100.68 & 96.42 & 57.18 & 54.63 \\
\hline & 13.0 & 55.00 & 57.44 & 96.78 & 101.14 & 101.51 & 101.51 & 101.14 & 96.77 & 57.42 & 54.84 \\
\hline & 14.0 & 55.16 & 57.61 & 98.10 & 102.63 & 103.01 & 103.01 & 102.63 & 98.10 & 57.60 & 55.00 \\
\hline & 15.0 & 55.00 & 54.21 & 55.12 & 55.71 & 56.07 & 56.07 & 55.70 & 55.12 & 54.20 & 54.81 \\
\hline \multirow{9}{*}{ A-3 } & 15.0 & 55.00 & 54.21 & 55.12 & 55.71 & 56.07 & 56.07 & 55.70 & 55.12 & 54.20 & 54.81 \\
\hline & 16.0 & 55.52 & 57.68 & 94.53 & 98.62 & 99.02 & 99.02 & 98.62 & 94.53 & 57.65 & 55.28 \\
\hline & 17.0 & 55.71 & 57.87 & 94.46 & 98.57 & 99.00 & 99.00 & 98.57 & 94.45 & 57.86 & 55.57 \\
\hline & 18.0 & 55.88 & 58.08 & 94.67 & 98.84 & 99.30 & 99.30 & 98.84 & 94.67 & 58.07 & 55.78 \\
\hline & 19.0 & 56.07 & 58.35 & 95.21 & 99.50 & 99.98 & 99.98 & 99.49 & 95.21 & 58.33 & 55.93 \\
\hline & 20.0 & 56.24 & 58.56 & 95.45 & 99.79 & 100.30 & 100.30 & 99.79 & 95.45 & 58.54 & 56.02 \\
\hline & 21.0 & 56.42 & 58.86 & 96.43 & 100.92 & 101.45 & 101.45 & 100.92 & 96.43 & 58.84 & 56.21 \\
\hline & 22.0 & 56.31 & 56.36 & 60.42 & 61.99 & 62.54 & 62.54 & 61.99 & 60.42 & 56.35 & 56.11 \\
\hline & 22.5 & 56.27 & 55.77 & 56.93 & 57.75 & 58.28 & 58.28 & 57.75 & 56.93 & 55.76 & 56.17 \\
\hline \multirow{9}{*}{ A-4 } & 22.5 & 56.27 & 55.77 & 56.93 & 57.75 & 58.28 & 58.28 & 57.75 & 56.93 & 55.76 & 56.17 \\
\hline & 23.0 & 56.44 & 56.58 & 60.88 & 62.52 & 63.07 & 63.07 & 62.52 & 60.87 & 56.56 & 56.24 \\
\hline & 24.0 & 56.87 & 59.72 & 100.74 & 105.59 & 106.16 & 106.16 & 105.59 & 100.74 & 59.70 & 56.62 \\
\hline & 25.0 & 57.00 & 59.93 & 100.68 & 105.54 & 106.13 & 106.13 & 105.54 & 100.68 & 59.90 & 56.78 \\
\hline & 26.0 & 57.16 & 60.18 & 100.98 & 105.87 & 106.49 & 106.49 & 105.87 & 100.98 & 60.16 & 57.00 \\
\hline & 27.0 & 57.29 & 60.40 & 101.09 & 106.00 & 106.64 & 106.64 & 106.00 & 101.09 & 60.38 & 57.16 \\
\hline & 28.0 & 57.41 & 60.62 & 101.34 & 106.27 & 106.93 & 106.93 & 106.27 & 101.33 & 60.59 & 57.24 \\
\hline & 29.0 & 57.48 & 60.74 & 101.59 & 106.50 & 107.16 & 107.16 & 106.49 & 101.58 & 60.71 & 57.25 \\
\hline & 30.0 & 57.27 & 57.48 & 58.98 & 60.03 & 60.71 & 60.71 & 60.03 & 58.97 & 57.45 & 57.03 \\
\hline \multirow{9}{*}{ A-5 } & 30.0 & 57.27 & 57.48 & 58.98 & 60.03 & 60.71 & 60.71 & 60.03 & 58.98 & 57.45 & 57.03 \\
\hline & 31.0 & 57.69 & 61.17 & 102.01 & 106.96 & 107.63 & 107.63 & 106.96 & 102.00 & 61.15 & 57.49 \\
\hline & 32.0 & 57.80 & 61.30 & 100.73 & 105.60 & 106.30 & 106.30 & 105.60 & 100.73 & 61.27 & 57.59 \\
\hline & 33.0 & 57.88 & 61.48 & 100.85 & 105.73 & 106.44 & 106.44 & 105.72 & 100.85 & 61.45 & 57.64 \\
\hline & 34.0 & 57.97 & 61.69 & 100.74 & 105.61 & 106.34 & 106.34 & 105.60 & 100.74 & 61.66 & 57.74 \\
\hline & 35.0 & 58.03 & 61.80 & 99.80 & 104.58 & 105.33 & 105.33 & 104.58 & 99.79 & 61.78 & 57.86 \\
\hline & 36.0 & 58.11 & 62.04 & 100.14 & 104.95 & 105.71 & 105.71 & 104.95 & 100.13 & 62.02 & 57.97 \\
\hline & 37.0 & 57.92 & 59.61 & 64.10 & 66.00 & 66.78 & 66.78 & 66.00 & 64.10 & 59.59 & 57.76 \\
\hline & 37.5 & 57.85 & 59.05 & 60.73 & 61.91 & 62.68 & 62.68 & 61.90 & 60.72 & 59.02 & 57.63 \\
\hline \multirow{9}{*}{ A-6 } & 37.5 & 57.85 & 59.05 & 60.73 & 61.91 & 62.68 & 62.68 & 61.90 & 60.72 & 59.02 & 57.63 \\
\hline & 38.0 & 57.94 & 59.66 & 63.80 & 65.60 & 66.37 & 66.37 & 65.59 & 63.79 & 59.63 & 57.73 \\
\hline & 39.0 & 58.19 & 62.20 & 96.97 & 101.46 & 102.22 & 102.21 & 101.45 & 96.96 & 62.17 & 57.97 \\
\hline & 40.0 & 58.20 & 62.14 & 94.07 & 98.30 & 99.07 & 99.07 & 98.30 & 94.06 & 62.11 & 58.01 \\
\hline & 41.0 & 58.21 & 62.16 & 92.15 & 96.21 & 96.98 & 96.97 & 96.20 & 92.14 & 62.13 & 58.02 \\
\hline & 42.0 & 58.21 & 62.15 & 90.00 & 93.86 & 94.64 & 94.64 & 93.86 & 89.99 & 62.12 & 58.00 \\
\hline & 43.0 & 58.21 & 62.24 & 89.85 & 93.66 & 94.44 & 94.44 & 93.66 & 89.84 & 62.21 & 58.01 \\
\hline & 44.0 & 58.15 & 62.16 & 87.74 & 91.28 & 92.06 & 92.06 & 91.28 & 87.74 & 62.14 & 58.03 \\
\hline & 45.0 & 57.95 & 60.24 & 61.92 & 63.11 & 63.90 & 63.89 & 63.10 & 61.92 & 60.22 & 57.87 \\
\hline
\end{tabular}


Table B - 23: Temperature $\left({ }^{\circ} \mathrm{C}\right)$ map of the east side of position B at MOC1 145A (total 69.7 EFPD).

\begin{tabular}{|c|c|c|c|c|c|c|c|c|c|c|c|}
\hline \multirow{2}{*}{$\begin{array}{c}\text { Plate } \\
\text { Location }\end{array}$} & \multirow{2}{*}{$\begin{array}{c}\text { Axial } \\
\text { Location (in) }\end{array}$} & \multicolumn{10}{|c|}{ Width of Plate (inches) } \\
\hline & & 0.00 & 0.08 & 0.16 & 0.25 & 0.50 & 1.00 & 1.50 & 2.00 & 2.08 & 2.16 \\
\hline \multirow{9}{*}{ B-1 } & 0.0 & 51.67 & 51.79 & 51.76 & 51.77 & 51.77 & 51.77 & 51.77 & 51.76 & 51.79 & 51.67 \\
\hline & 1.0 & 52.97 & 53.87 & 75.04 & 77.37 & 77.45 & 77.45 & 77.37 & 75.04 & 53.84 & 52.87 \\
\hline & 2.0 & 53.19 & 54.20 & 77.75 & 80.30 & 80.39 & 80.39 & 80.30 & 77.74 & 54.14 & 52.94 \\
\hline & 3.0 & 53.41 & 54.40 & 78.23 & 80.87 & 80.98 & 80.98 & 80.87 & 78.23 & 54.34 & 53.03 \\
\hline & 4.0 & 53.67 & 54.76 & 80.84 & 83.70 & 83.83 & 83.83 & 83.70 & 80.84 & 54.70 & 53.32 \\
\hline & 5.0 & 53.90 & 55.16 & 84.03 & 87.14 & 87.30 & 87.30 & 87.14 & 84.02 & 55.10 & 53.49 \\
\hline & 6.0 & 54.18 & 55.63 & 87.46 & 90.86 & 91.04 & 91.04 & 90.86 & 87.46 & 55.55 & 53.68 \\
\hline & 7.0 & 54.16 & 53.42 & 56.20 & 57.13 & 57.31 & 57.31 & 57.13 & 56.19 & 53.33 & 53.64 \\
\hline & 7.5 & 54.18 & 52.93 & 53.24 & 53.50 & 53.66 & 53.66 & 53.50 & 53.23 & 52.83 & 53.63 \\
\hline \multirow{9}{*}{ B-2 } & 7.5 & 54.18 & 52.93 & 53.24 & 53.50 & 53.66 & 53.66 & 53.50 & 53.23 & 52.83 & 53.63 \\
\hline & 8.0 & 54.37 & 53.59 & 56.59 & 57.60 & 57.79 & 57.79 & 57.59 & 56.59 & 53.49 & 53.79 \\
\hline & 9.0 & 54.85 & 56.33 & 90.87 & 94.54 & 94.76 & 94.76 & 94.54 & 90.87 & 56.30 & 54.76 \\
\hline & 10.0 & 55.07 & 56.57 & 91.65 & 95.39 & 95.63 & 95.63 & 95.38 & 91.64 & 56.51 & 54.82 \\
\hline & 11.0 & 55.33 & 56.90 & 92.99 & 96.84 & 97.11 & 97.11 & 96.84 & 92.99 & 56.79 & 54.83 \\
\hline & 12.0 & 55.55 & 57.16 & 94.01 & 97.94 & 98.26 & 98.26 & 97.94 & 94.00 & 57.05 & 54.86 \\
\hline & 13.0 & 55.77 & 57.41 & 94.38 & 98.40 & 98.76 & 98.76 & 98.40 & 94.38 & 57.30 & 55.13 \\
\hline & 14.0 & 55.96 & 57.64 & 96.14 & 100.38 & 100.75 & 100.75 & 100.38 & 96.13 & 57.52 & 55.27 \\
\hline & 15.0 & 55.83 & 54.43 & 55.15 & 55.72 & 56.07 & 56.07 & 55.72 & 55.14 & 54.30 & 55.10 \\
\hline \multirow{9}{*}{ B-3 } & 15.0 & 55.83 & 54.43 & 55.15 & 55.72 & 56.07 & 56.07 & 55.72 & 55.14 & 54.30 & 55.10 \\
\hline & 16.0 & 56.39 & 58.22 & 98.07 & 102.63 & 103.04 & 103.04 & 102.63 & 98.06 & 58.08 & 55.57 \\
\hline & 17.0 & 56.59 & 58.45 & 98.23 & 102.82 & 103.25 & 103.25 & 102.82 & 98.22 & 58.36 & 56.26 \\
\hline & 18.0 & 56.78 & 58.67 & 98.49 & 103.12 & 103.58 & 103.58 & 103.12 & 98.48 & 58.62 & 56.61 \\
\hline & 19.0 & 57.00 & 58.94 & 98.98 & 103.67 & 104.16 & 104.16 & 103.67 & 98.97 & 58.84 & 56.63 \\
\hline & 20.0 & 57.17 & 59.16 & 99.28 & 104.01 & 104.52 & 104.52 & 104.00 & 99.27 & 59.01 & 56.50 \\
\hline & 21.0 & 57.37 & 59.44 & 99.99 & 104.78 & 105.32 & 105.32 & 104.78 & 99.98 & 59.30 & 56.61 \\
\hline & 22.0 & 57.26 & 56.72 & 60.90 & 62.53 & 63.08 & 63.08 & 62.53 & 60.89 & 56.58 & 56.46 \\
\hline & 22.5 & 57.22 & 56.08 & 57.09 & 57.91 & 58.43 & 58.43 & 57.90 & 57.08 & 56.02 & 57.10 \\
\hline \multirow{9}{*}{ B-4 } & 22.5 & 57.22 & 56.08 & 57.10 & 57.91 & 58.43 & 58.43 & 57.90 & 57.08 & 56.02 & 57.10 \\
\hline & 23.0 & 57.39 & 56.88 & 61.05 & 62.68 & 63.23 & 63.23 & 62.68 & 61.03 & 56.74 & 56.59 \\
\hline & 24.0 & 57.82 & 60.04 & 101.06 & 105.92 & 106.49 & 106.48 & 105.92 & 101.05 & 59.88 & 56.92 \\
\hline & 25.0 & 57.96 & 60.19 & 100.41 & 105.23 & 105.82 & 105.82 & 105.23 & 100.40 & 60.04 & 57.26 \\
\hline & 26.0 & 58.11 & 60.46 & 100.75 & 105.60 & 106.22 & 106.22 & 105.60 & 100.74 & 60.35 & 57.77 \\
\hline & 27.0 & 58.24 & 60.68 & 100.91 & 105.79 & 106.43 & 106.42 & 105.79 & 100.90 & 60.62 & 58.04 \\
\hline & 28.0 & 58.35 & 60.90 & 101.17 & 106.07 & 106.73 & 106.73 & 106.07 & 101.16 & 60.80 & 57.97 \\
\hline & 29.0 & 58.42 & 61.03 & 101.50 & 106.39 & 107.04 & 107.04 & 106.38 & 101.49 & 60.88 & 57.72 \\
\hline & 30.0 & 58.19 & 57.81 & 59.15 & 60.18 & 60.85 & 60.85 & 60.17 & 59.13 & 57.65 & 57.36 \\
\hline \multirow{9}{*}{ B-5 } & 30.0 & 58.19 & 57.81 & 59.15 & 60.18 & 60.86 & 60.85 & 60.17 & 59.13 & 57.65 & 57.36 \\
\hline & 31.0 & 58.59 & 61.26 & 99.44 & 104.17 & 104.83 & 104.83 & 104.16 & 99.43 & 61.13 & 57.90 \\
\hline & 32.0 & 58.69 & 61.40 & 98.37 & 103.03 & 103.72 & 103.72 & 103.02 & 98.36 & 61.26 & 57.91 \\
\hline & 33.0 & 58.75 & 61.58 & 98.50 & 103.16 & 103.87 & 103.87 & 103.15 & 98.49 & 61.43 & 57.89 \\
\hline & 34.0 & 58.82 & 61.80 & 98.40 & 103.05 & 103.77 & 103.77 & 103.04 & 98.38 & 61.65 & 58.13 \\
\hline & 35.0 & 58.86 & 61.91 & 97.49 & 102.06 & 102.80 & 102.80 & 102.06 & 97.47 & 61.80 & 58.49 \\
\hline & 36.0 & 58.91 & 62.11 & 97.41 & 101.97 & 102.72 & 102.72 & 101.96 & 97.39 & 62.04 & 58.63 \\
\hline & 37.0 & 58.70 & 59.88 & 64.00 & 65.81 & 66.58 & 66.58 & 65.80 & 63.97 & 59.77 & 58.41 \\
\hline & 37.5 & 58.62 & 59.36 & 60.89 & 62.04 & 62.80 & 62.80 & 62.03 & 60.87 & 59.21 & 57.88 \\
\hline \multirow{9}{*}{ B-6 } & 37.5 & 58.62 & 59.36 & 60.89 & 62.04 & 62.80 & 62.80 & 62.03 & 60.87 & 59.21 & 57.88 \\
\hline & 38.0 & 58.70 & 59.96 & 63.88 & 65.63 & 66.39 & 66.39 & 65.62 & 63.85 & 59.81 & 58.07 \\
\hline & 39.0 & 58.91 & 62.42 & 96.14 & 100.54 & 101.29 & 101.29 & 100.53 & 96.13 & 62.29 & 58.23 \\
\hline & 40.0 & 58.89 & 62.37 & 93.47 & 97.62 & 98.39 & 98.39 & 97.62 & 93.45 & 62.24 & 58.22 \\
\hline & 41.0 & 58.87 & 62.37 & 91.38 & 95.34 & 96.10 & 96.10 & 95.33 & 91.36 & 62.25 & 58.26 \\
\hline & 42.0 & 58.83 & 62.37 & 89.30 & 93.05 & 93.83 & 93.82 & 93.04 & 89.28 & 62.24 & 58.15 \\
\hline & 43.0 & 58.80 & 62.44 & 89.15 & 92.86 & 93.63 & 93.63 & 92.85 & 89.14 & 62.32 & 58.27 \\
\hline & 44.0 & 58.70 & 62.37 & 87.11 & 90.55 & 91.32 & 91.32 & 90.54 & 87.10 & 62.29 & 58.45 \\
\hline & 45.0 & 58.48 & 60.51 & 62.12 & 63.28 & 64.05 & 64.05 & 63.26 & 62.09 & 60.46 & 58.35 \\
\hline
\end{tabular}


Table B - 24: Temperature $\left({ }^{\circ} \mathrm{C}\right)$ map of the west side of position B at MOC1 145A (total 69.7 EFPD).

\begin{tabular}{|c|c|c|c|c|c|c|c|c|c|c|c|}
\hline \multirow{2}{*}{$\begin{array}{c}\text { Plate } \\
\text { Location }\end{array}$} & \multirow{2}{*}{$\begin{array}{c}\text { Axial } \\
\text { Location (in) }\end{array}$} & \multicolumn{10}{|c|}{ Width of Plate (inches) } \\
\hline & & 0.00 & 0.08 & 0.16 & 0.25 & 0.50 & 1.00 & 1.50 & 2.00 & 2.08 & 2.16 \\
\hline \multirow{9}{*}{ B-1 } & 0.0 & 51.67 & 51.79 & 51.76 & 51.77 & 51.77 & 51.77 & 51.77 & 51.76 & 51.79 & 51.67 \\
\hline & 1.0 & 53.29 & 53.85 & 75.03 & 77.36 & 77.43 & 77.43 & 77.36 & 75.03 & 53.83 & 53.17 \\
\hline & 2.0 & 53.54 & 54.16 & 77.72 & 80.27 & 80.35 & 80.35 & 80.27 & 77.72 & 54.12 & 53.10 \\
\hline & 3.0 & 53.79 & 54.35 & 78.18 & 80.81 & 80.91 & 80.91 & 80.81 & 78.18 & 54.31 & 53.06 \\
\hline & 4.0 & 54.08 & 54.69 & 80.75 & 83.59 & 83.71 & 83.71 & 83.59 & 80.75 & 54.65 & 53.39 \\
\hline & 5.0 & 54.34 & 55.07 & 83.90 & 86.99 & 87.12 & 87.12 & 86.99 & 83.90 & 55.02 & 53.51 \\
\hline & 6.0 & 54.64 & 55.51 & 87.30 & 90.65 & 90.80 & 90.80 & 90.65 & 87.30 & 55.45 & 53.71 \\
\hline & 7.0 & 54.68 & 53.28 & 56.03 & 56.92 & 57.07 & 57.07 & 56.92 & 56.02 & 53.21 & 53.76 \\
\hline & 7.5 & 54.73 & 52.77 & 53.05 & 53.27 & 53.39 & 53.39 & 53.27 & 53.04 & 52.70 & 53.77 \\
\hline \multirow{9}{*}{ B-2 } & 7.5 & 54.73 & 52.77 & 53.05 & 53.27 & 53.39 & 53.39 & 53.27 & 53.04 & 52.70 & 53.77 \\
\hline & 8.0 & 54.92 & 53.43 & 56.40 & 57.35 & 57.51 & 57.51 & 57.35 & 56.39 & 53.36 & 53.91 \\
\hline & 9.0 & 55.39 & 56.15 & 90.62 & 94.22 & 94.39 & 94.39 & 94.22 & 90.61 & 56.12 & 55.42 \\
\hline & 10.0 & 55.63 & 56.37 & 91.35 & 95.02 & 95.21 & 95.21 & 95.01 & 91.34 & 56.32 & 55.36 \\
\hline & 11.0 & 55.91 & 56.66 & 92.64 & 96.41 & 96.62 & 96.62 & 96.41 & 92.63 & 56.58 & 55.05 \\
\hline & 12.0 & 56.15 & 56.89 & 93.60 & 97.44 & 97.67 & 97.67 & 97.44 & 93.60 & 56.81 & 54.83 \\
\hline & 13.0 & 56.38 & 57.11 & 93.92 & 97.81 & 98.06 & 98.06 & 97.81 & 93.91 & 57.03 & 55.13 \\
\hline & 14.0 & 56.58 & 57.30 & 95.63 & 99.65 & 99.93 & 99.92 & 99.64 & 95.62 & 57.21 & 55.23 \\
\hline & 15.0 & 56.52 & 54.05 & 54.65 & 55.10 & 55.37 & 55.37 & 55.10 & 54.63 & 53.96 & 55.21 \\
\hline \multirow{9}{*}{ B-3 } & 15.0 & 56.52 & 54.05 & 54.65 & 55.10 & 55.37 & 55.37 & 55.10 & 54.63 & 53.96 & 55.21 \\
\hline & 16.0 & 57.04 & 57.83 & 97.47 & 101.80 & 102.10 & 102.10 & 101.80 & 97.46 & 57.71 & 55.62 \\
\hline & 17.0 & 57.24 & 58.03 & 97.56 & 101.94 & 102.26 & 102.26 & 101.93 & 97.55 & 57.96 & 56.87 \\
\hline & 18.0 & 57.44 & 58.22 & 97.75 & 102.18 & 102.52 & 102.52 & 102.17 & 97.74 & 58.17 & 57.41 \\
\hline & 19.0 & 57.65 & 58.45 & 98.15 & 102.66 & 103.02 & 103.02 & 102.65 & 98.14 & 58.37 & 57.21 \\
\hline & 20.0 & 57.83 & 58.64 & 98.39 & 102.93 & 103.30 & 103.30 & 102.92 & 98.37 & 58.53 & 56.71 \\
\hline & 21.0 & 58.01 & 58.88 & 99.04 & 103.63 & 104.02 & 104.02 & 103.62 & 99.02 & 58.76 & 56.63 \\
\hline & 22.0 & 57.94 & 56.12 & 60.12 & 61.58 & 62.01 & 62.00 & 61.58 & 60.10 & 56.01 & 56.39 \\
\hline & 22.5 & 57.92 & 55.46 & 56.28 & 56.92 & 57.32 & 57.32 & 56.92 & 56.26 & 55.40 & 58.04 \\
\hline \multirow{9}{*}{ B-4 } & 22.5 & 57.92 & 55.46 & 56.28 & 56.92 & 57.32 & 57.32 & 56.92 & 56.26 & 55.40 & 58.04 \\
\hline & 23.0 & 58.07 & 56.25 & 60.23 & 61.70 & 62.12 & 62.12 & 61.69 & 60.21 & 56.15 & 56.51 \\
\hline & 24.0 & 58.46 & 59.39 & 99.98 & 104.62 & 105.03 & 105.03 & 104.61 & 99.96 & 59.26 & 56.77 \\
\hline & 25.0 & 58.57 & 59.52 & 99.27 & 103.87 & 104.29 & 104.29 & 103.86 & 99.25 & 59.39 & 57.40 \\
\hline & 26.0 & 58.70 & 59.74 & 99.54 & 104.16 & 104.60 & 104.60 & 104.15 & 99.52 & 59.64 & 58.37 \\
\hline & 27.0 & 58.82 & 59.92 & 99.64 & 104.28 & 104.73 & 104.73 & 104.27 & 99.62 & 59.85 & 58.76 \\
\hline & 28.0 & 58.91 & 60.10 & 99.83 & 104.48 & 104.95 & 104.94 & 104.47 & 99.81 & 60.01 & 58.47 \\
\hline & 29.0 & 58.95 & 60.19 & 100.09 & 104.71 & 105.18 & 105.17 & 104.70 & 100.07 & 60.06 & 57.78 \\
\hline & 30.0 & 58.75 & 56.91 & 57.98 & 58.79 & 59.30 & 59.30 & 58.78 & 57.95 & 56.78 & 57.25 \\
\hline \multirow{9}{*}{ B-5 } & 30.0 & 58.75 & 56.91 & 57.98 & 58.79 & 59.30 & 59.30 & 58.78 & 57.95 & 56.78 & 57.25 \\
\hline & 31.0 & 59.08 & 60.35 & 97.93 & 102.38 & 102.85 & 102.84 & 102.37 & 97.90 & 60.23 & 57.78 \\
\hline & 32.0 & 59.14 & 60.46 & 96.80 & 101.18 & 101.66 & 101.66 & 101.16 & 96.77 & 60.33 & 57.64 \\
\hline & 33.0 & 59.18 & 60.60 & 96.88 & 101.25 & 101.74 & 101.74 & 101.24 & 96.85 & 60.47 & 57.57 \\
\hline & 34.0 & 59.20 & 60.77 & 96.70 & 101.05 & 101.56 & 101.55 & 101.04 & 96.67 & 60.63 & 58.05 \\
\hline & 35.0 & 59.22 & 60.84 & 95.72 & 99.99 & 100.50 & 100.50 & 99.97 & 95.69 & 60.73 & 58.79 \\
\hline & 36.0 & 59.22 & 60.99 & 95.56 & 99.81 & 100.33 & 100.33 & 99.79 & 95.53 & 60.91 & 58.94 \\
\hline & 37.0 & 59.01 & 58.71 & 62.51 & 64.08 & 64.66 & 64.65 & 64.06 & 62.48 & 58.60 & 58.77 \\
\hline & 37.5 & 58.93 & 58.17 & 59.38 & 60.26 & 60.83 & 60.82 & 60.24 & 59.34 & 58.04 & 57.61 \\
\hline \multirow{9}{*}{ B-6 } & 37.5 & 58.93 & 58.17 & 59.38 & 60.26 & 60.83 & 60.82 & 60.24 & 59.34 & 58.04 & 57.61 \\
\hline & 38.0 & 58.98 & 58.76 & 62.37 & 63.87 & 64.43 & 64.43 & 63.85 & 62.33 & 58.62 & 57.94 \\
\hline & 39.0 & 59.11 & 61.20 & 94.18 & 98.23 & 98.74 & 98.74 & 98.21 & 94.15 & 61.07 & 57.89 \\
\hline & 40.0 & 59.06 & 61.12 & 91.54 & 95.24 & 95.77 & 95.76 & 95.23 & 91.50 & 60.99 & 57.76 \\
\hline & 41.0 & 58.99 & 61.08 & 89.43 & 92.91 & 93.40 & 93.39 & 92.90 & 89.40 & 60.95 & 57.87 \\
\hline & 42.0 & 58.91 & 61.04 & 87.31 & 90.63 & 91.12 & 91.11 & 90.61 & 87.27 & 60.90 & 57.66 \\
\hline & 43.0 & 58.84 & 61.08 & 87.12 & 90.38 & 90.87 & 90.87 & 90.36 & 87.08 & 60.95 & 57.96 \\
\hline & 44.0 & 58.70 & 60.97 & 85.00 & 88.06 & 88.54 & 88.54 & 88.04 & 84.96 & 60.87 & 58.36 \\
\hline & 45.0 & 58.48 & 59.08 & 60.33 & 61.22 & 61.79 & 61.78 & 61.19 & 60.29 & 59.00 & 58.35 \\
\hline
\end{tabular}


Table B - 25: Temperature $\left({ }^{\circ} \mathrm{C}\right)$ map of the east side of position A at MOC2 145A (total 89.7 EFPD).

\begin{tabular}{|c|c|c|c|c|c|c|c|c|c|c|c|}
\hline \multirow{2}{*}{$\begin{array}{c}\text { Plate } \\
\text { Location }\end{array}$} & \multirow{2}{*}{$\begin{array}{c}\text { Axial } \\
\text { Location (in) }\end{array}$} & \multicolumn{10}{|c|}{ Width of Plate (inches) } \\
\hline & & 0.00 & 0.08 & 0.16 & 0.25 & 0.50 & 1.00 & 1.50 & 2.00 & 2.08 & 2.16 \\
\hline \multirow{9}{*}{ A-1 } & 0.0 & 51.67 & 51.77 & 51.76 & 51.77 & 51.77 & 51.77 & 51.77 & 51.76 & 51.77 & 51.67 \\
\hline & 1.0 & 52.51 & 53.89 & 75.99 & 78.40 & 78.47 & 78.47 & 78.40 & 75.99 & 53.88 & 52.49 \\
\hline & 2.0 & 52.68 & 54.21 & 78.94 & 81.59 & 81.68 & 81.68 & 81.59 & 78.94 & 54.20 & 52.64 \\
\hline & 3.0 & 52.84 & 54.40 & 79.40 & 82.14 & 82.24 & 82.24 & 82.14 & 79.40 & 54.39 & 52.80 \\
\hline & 4.0 & 53.03 & 54.70 & 81.76 & 84.68 & 84.81 & 84.81 & 84.68 & 81.76 & 54.70 & 53.00 \\
\hline & 5.0 & 53.20 & 55.03 & 84.32 & 87.45 & 87.58 & 87.58 & 87.45 & 84.32 & 55.02 & 53.16 \\
\hline & 6.0 & 53.40 & 55.35 & 86.52 & 89.83 & 89.98 & 89.98 & 89.83 & 86.52 & 55.34 & 53.34 \\
\hline & 7.0 & 53.39 & 53.15 & 55.92 & 56.81 & 56.96 & 56.96 & 56.81 & 55.92 & 53.13 & 53.31 \\
\hline & 7.5 & 53.39 & 52.65 & 53.02 & 53.25 & 53.37 & 53.37 & 53.25 & 53.01 & 52.63 & 53.31 \\
\hline \multirow{9}{*}{ A-2 } & 7.5 & 53.39 & 52.65 & 53.02 & 53.25 & 53.37 & 53.37 & 53.25 & 53.01 & 52.63 & 53.31 \\
\hline & 8.0 & 53.54 & 53.34 & 56.51 & 57.51 & 57.67 & 57.67 & 57.51 & 56.51 & 53.32 & 53.46 \\
\hline & 9.0 & 53.93 & 56.15 & 92.05 & 95.78 & 95.95 & 95.95 & 95.78 & 92.05 & 56.14 & 53.89 \\
\hline & 10.0 & 54.10 & 56.35 & 92.77 & 96.55 & 96.75 & 96.75 & 96.55 & 92.76 & 56.34 & 54.05 \\
\hline & 11.0 & 54.30 & 56.56 & 93.34 & 97.18 & 97.39 & 97.39 & 97.18 & 93.34 & 56.55 & 54.22 \\
\hline & 12.0 & 54.47 & 56.78 & 94.28 & 98.19 & 98.43 & 98.43 & 98.19 & 94.28 & 56.76 & 54.39 \\
\hline & 13.0 & 54.64 & 56.96 & 94.52 & 98.48 & 98.73 & 98.73 & 98.48 & 94.52 & 56.95 & 54.57 \\
\hline & 14.0 & 54.79 & 57.06 & 95.32 & 99.31 & 99.59 & 99.59 & 99.31 & 95.31 & 57.04 & 54.71 \\
\hline & 15.0 & 54.69 & 53.81 & 54.51 & 54.98 & 55.26 & 55.26 & 54.98 & 54.51 & 53.78 & 54.59 \\
\hline \multirow{9}{*}{ A-3 } & 15.0 & 54.69 & 53.81 & 54.51 & 54.98 & 55.26 & 55.26 & 54.98 & 54.51 & 53.78 & 54.59 \\
\hline & 16.0 & 55.10 & 57.00 & 91.18 & 94.87 & 95.13 & 95.13 & 94.87 & 91.18 & 56.98 & 54.98 \\
\hline & 17.0 & 55.27 & 57.19 & 91.44 & 95.16 & 95.43 & 95.43 & 95.16 & 91.44 & 57.17 & 55.20 \\
\hline & 18.0 & 55.42 & 57.35 & 91.61 & 95.34 & 95.64 & 95.64 & 95.34 & 91.61 & 57.34 & 55.37 \\
\hline & 19.0 & 55.60 & 57.55 & 91.93 & 95.70 & 96.01 & 96.01 & 95.70 & 91.93 & 57.53 & 55.52 \\
\hline & 20.0 & 55.74 & 57.68 & 91.74 & 95.50 & 95.83 & 95.83 & 95.50 & 91.74 & 57.65 & 55.64 \\
\hline & 21.0 & 55.90 & 57.86 & 92.04 & 95.83 & 96.17 & 96.17 & 95.83 & 92.04 & 57.84 & 55.80 \\
\hline & 22.0 & 55.84 & 55.54 & 59.02 & 60.34 & 60.75 & 60.75 & 60.34 & 59.02 & 55.51 & 55.75 \\
\hline & 22.5 & 55.82 & 54.98 & 55.82 & 56.44 & 56.82 & 56.82 & 56.44 & 55.81 & 54.96 & 55.77 \\
\hline \multirow{9}{*}{ A-4 } & 22.5 & 55.82 & 54.98 & 55.82 & 56.44 & 56.82 & 56.82 & 56.44 & 55.81 & 54.96 & 55.77 \\
\hline & 23.0 & 55.96 & 55.71 & 59.46 & 60.85 & 61.26 & 61.26 & 60.84 & 59.45 & 55.69 & 55.87 \\
\hline & 24.0 & 56.30 & 58.60 & 96.20 & 100.46 & 100.86 & 100.86 & 100.46 & 96.20 & 58.58 & 56.19 \\
\hline & 25.0 & 56.42 & 58.76 & 96.18 & 100.46 & 100.88 & 100.88 & 100.46 & 96.18 & 58.74 & 56.31 \\
\hline & 26.0 & 56.56 & 58.94 & 96.26 & 100.57 & 101.00 & 101.00 & 100.57 & 96.26 & 58.92 & 56.48 \\
\hline & 27.0 & 56.67 & 59.13 & 96.75 & 101.14 & 101.58 & 101.58 & 101.14 & 96.75 & 59.12 & 56.61 \\
\hline & 28.0 & 56.78 & 59.29 & 96.90 & 101.32 & 101.78 & 101.78 & 101.32 & 96.90 & 59.27 & 56.70 \\
\hline & 29.0 & 56.84 & 59.32 & 96.67 & 101.03 & 101.49 & 101.49 & 101.03 & 96.67 & 59.30 & 56.73 \\
\hline & 30.0 & 56.69 & 56.25 & 57.33 & 58.11 & 58.61 & 58.61 & 58.11 & 57.33 & 56.22 & 56.58 \\
\hline \multirow{9}{*}{ A-5 } & 30.0 & 56.69 & 56.25 & 57.33 & 58.11 & 58.61 & 58.61 & 58.11 & 57.33 & 56.22 & 56.58 \\
\hline & 31.0 & 57.03 & 59.66 & 97.03 & 101.46 & 101.92 & 101.92 & 101.46 & 97.03 & 59.64 & 56.94 \\
\hline & 32.0 & 57.13 & 59.83 & 97.02 & 101.45 & 101.93 & 101.93 & 101.45 & 97.02 & 59.81 & 57.03 \\
\hline & 33.0 & 57.20 & 59.99 & 97.16 & 101.61 & 102.10 & 102.10 & 101.61 & 97.16 & 59.96 & 57.09 \\
\hline & 34.0 & 57.28 & 60.14 & 97.03 & 101.47 & 101.97 & 101.97 & 101.47 & 97.03 & 60.12 & 57.17 \\
\hline & 35.0 & 57.33 & 60.19 & 96.04 & 100.38 & 100.89 & 100.89 & 100.38 & 96.03 & 60.18 & 57.26 \\
\hline & 36.0 & 57.39 & 60.34 & 95.89 & 100.22 & 100.74 & 100.74 & 100.22 & 95.89 & 60.32 & 57.33 \\
\hline & 37.0 & 57.25 & 57.96 & 61.90 & 63.47 & 64.05 & 64.05 & 63.47 & 61.89 & 57.94 & 57.17 \\
\hline & 37.5 & 57.19 & 57.40 & 58.64 & 59.52 & 60.08 & 60.08 & 59.52 & 58.63 & 57.38 & 57.09 \\
\hline \multirow{9}{*}{ A-6 } & 37.5 & 57.19 & 57.40 & 58.64 & 59.52 & 60.08 & 60.08 & 59.52 & 58.63 & 57.38 & 57.09 \\
\hline & 38.0 & 57.27 & 57.98 & 61.65 & 63.15 & 63.71 & 63.71 & 63.15 & 61.64 & 57.96 & 57.17 \\
\hline & 39.0 & 57.47 & 60.44 & 93.61 & 97.61 & 98.12 & 98.12 & 97.61 & 93.61 & 60.42 & 57.37 \\
\hline & $\begin{array}{l}40.0 \\
\end{array}$ & 57.48 & 60.42 & 91.76 & 95.53 & 96.05 & 96.05 & 95.53 & 91.76 & 60.40 & 57.40 \\
\hline & 41.0 & 57.49 & 60.39 & 89.84 & 93.43 & 93.91 & 93.91 & 93.43 & 89.84 & 60.38 & 57.42 \\
\hline & 42.0 & 57.49 & 60.34 & 87.70 & 91.13 & 91.62 & 91.62 & 91.13 & 87.70 & 60.32 & 57.40 \\
\hline & 43.0 & 57.49 & 60.38 & 87.51 & 90.88 & 91.37 & 91.37 & 90.88 & 87.51 & 60.37 & 57.40 \\
\hline & 44.0 & 57.44 & 60.26 & 85.36 & 88.52 & 89.02 & 89.02 & 88.52 & 85.36 & 60.25 & 57.39 \\
\hline & 45.0 & 57.26 & 58.27 & 59.55 & 60.44 & 61.02 & 61.02 & 60.44 & 59.55 & 58.26 & 57.24 \\
\hline
\end{tabular}


Table B - 26: Temperature $\left({ }^{\circ} \mathrm{C}\right)$ map of the west side of position A at MOC2 145A (total 89.7 EFPD).

\begin{tabular}{|c|c|c|c|c|c|c|c|c|c|c|c|}
\hline \multirow{2}{*}{$\begin{array}{c}\text { Plate } \\
\text { Location }\end{array}$} & \multirow{2}{*}{$\begin{array}{c}\text { Axial } \\
\text { Location (in) }\end{array}$} & \multicolumn{10}{|c|}{ Width of Plate (inches) } \\
\hline & & 0.00 & 0.08 & 0.16 & 0.25 & 0.50 & 1.00 & 1.50 & 2.00 & 2.08 & 2.16 \\
\hline \multirow{9}{*}{ A-1 } & 0.0 & 51.67 & 51.77 & 51.76 & 51.77 & 51.77 & 51.77 & 51.77 & 51.76 & 51.77 & 51.67 \\
\hline & 1.0 & 52.63 & 53.88 & 76.00 & 78.41 & 78.49 & 78.49 & 78.41 & 76.00 & 53.88 & 52.60 \\
\hline & 2.0 & 52.82 & 54.22 & 78.97 & 81.63 & 81.73 & 81.73 & 81.63 & 78.97 & 54.21 & 52.75 \\
\hline & 3.0 & 53.00 & 54.42 & 79.46 & 82.21 & 82.32 & 82.32 & 82.21 & 79.46 & 54.42 & 52.91 \\
\hline & 4.0 & 53.21 & 54.75 & 81.87 & 84.81 & 84.95 & 84.95 & 84.81 & 81.87 & 54.74 & 53.13 \\
\hline & 5.0 & 53.40 & 55.10 & 84.46 & 87.62 & 87.77 & 87.77 & 87.62 & 84.46 & 55.09 & 53.31 \\
\hline & 6.0 & 53.62 & 55.45 & 86.71 & 90.06 & 90.24 & 90.24 & 90.06 & 86.71 & 55.44 & 53.50 \\
\hline & 7.0 & 53.58 & 53.28 & 56.12 & 57.04 & 57.23 & 57.23 & 57.04 & 56.12 & 53.27 & 53.44 \\
\hline & 7.5 & 53.58 & 52.79 & 53.23 & 53.50 & 53.67 & 53.67 & 53.50 & 53.23 & 52.78 & 53.43 \\
\hline \multirow{9}{*}{ A-2 } & 7.5 & 53.58 & 52.79 & 53.23 & 53.50 & 53.67 & 53.67 & 53.50 & 53.23 & 52.78 & 53.43 \\
\hline & 8.0 & 53.75 & 53.48 & 56.73 & 57.78 & 57.97 & 57.97 & 57.78 & 56.73 & 53.47 & 53.59 \\
\hline & 9.0 & 54.20 & 56.31 & 92.35 & 96.13 & 96.35 & 96.35 & 96.13 & 92.35 & 56.30 & 54.13 \\
\hline & 10.0 & 54.38 & 56.54 & 93.10 & 96.95 & 97.20 & 97.20 & 96.95 & 93.10 & 56.53 & 54.28 \\
\hline & 11.0 & 54.60 & 56.79 & 93.74 & 97.65 & 97.92 & 97.92 & 97.65 & 93.74 & 56.78 & 54.45 \\
\hline & 12.0 & 54.79 & 57.04 & 94.73 & 98.75 & 99.08 & 99.08 & 98.75 & 94.72 & 57.03 & 54.62 \\
\hline & 13.0 & 54.98 & 57.26 & 95.03 & 99.14 & 99.49 & 99.49 & 99.14 & 95.03 & 57.25 & 54.82 \\
\hline & 14.0 & 55.14 & 57.40 & 95.88 & 100.09 & 100.46 & 100.46 & 100.08 & 95.88 & 57.38 & 54.97 \\
\hline & 15.0 & 54.99 & 54.18 & 55.06 & 55.63 & 55.99 & 55.99 & 55.63 & 55.06 & 54.17 & 54.80 \\
\hline \multirow{9}{*}{ A-3 } & 15.0 & 54.99 & 54.18 & 55.06 & 55.63 & 55.99 & 55.99 & 55.63 & 55.06 & 54.17 & 54.80 \\
\hline & 16.0 & 55.48 & 57.41 & 91.85 & 95.66 & 96.01 & 96.01 & 95.66 & 91.85 & 57.38 & 55.24 \\
\hline & 17.0 & 55.67 & 57.62 & 92.15 & 95.99 & 96.37 & 96.37 & 95.99 & 92.15 & 57.61 & 55.53 \\
\hline & 18.0 & 55.83 & 57.83 & 92.37 & 96.24 & 96.67 & 96.67 & 96.24 & 92.37 & 57.81 & 55.73 \\
\hline & 19.0 & 56.02 & 58.07 & 92.76 & 96.70 & 97.17 & 97.17 & 96.70 & 92.76 & 58.05 & 55.88 \\
\hline & 20.0 & 56.18 & 58.24 & 92.64 & 96.59 & 97.07 & 97.07 & 96.59 & 92.64 & 58.22 & 55.97 \\
\hline & 21.0 & 56.35 & 58.47 & 93.01 & 97.03 & 97.53 & 97.53 & 97.03 & 93.01 & 58.45 & 56.14 \\
\hline & 22.0 & 56.26 & 56.19 & 59.91 & 61.38 & 61.90 & 61.90 & 61.38 & 59.91 & 56.18 & 56.06 \\
\hline & 22.5 & 56.23 & 55.66 & 56.74 & 57.51 & 58.02 & 58.01 & 57.51 & 56.73 & 55.64 & 56.13 \\
\hline \multirow{9}{*}{ A-4 } & 22.5 & 56.23 & 55.66 & 56.74 & 57.52 & 58.02 & 58.02 & 57.51 & 56.73 & 55.64 & 56.13 \\
\hline & 23.0 & 56.39 & 56.40 & 60.38 & 61.92 & 62.45 & 62.45 & 61.92 & 60.38 & 56.38 & 56.19 \\
\hline & 24.0 & 56.79 & 59.32 & 97.36 & 101.90 & 102.45 & 102.45 & 101.90 & 97.36 & 59.30 & 56.54 \\
\hline & 25.0 & 56.92 & 59.52 & 97.41 & 101.97 & 102.54 & 102.54 & 101.97 & 97.41 & 59.49 & 56.69 \\
\hline & 26.0 & 57.06 & 59.75 & 97.58 & 102.16 & 102.75 & 102.75 & 102.16 & 97.58 & 59.73 & 56.91 \\
\hline & 27.0 & 57.19 & 59.98 & 98.17 & 102.80 & 103.41 & 103.41 & 102.80 & 98.16 & 59.96 & 57.07 \\
\hline & 28.0 & 57.30 & 60.19 & 98.40 & 103.07 & 103.69 & 103.69 & 103.06 & 98.40 & 60.17 & 57.14 \\
\hline & 29.0 & 57.37 & 60.27 & 98.25 & 102.86 & 103.49 & 103.49 & 102.86 & 98.25 & 60.25 & 57.15 \\
\hline & 30.0 & 57.17 & 57.24 & 58.63 & 59.61 & 60.25 & 60.25 & 59.61 & 58.62 & 57.22 & 56.94 \\
\hline \multirow{9}{*}{ A-5 } & 30.0 & 57.17 & 57.24 & 58.63 & 59.61 & 60.25 & 60.25 & 59.61 & 58.62 & 57.22 & 56.94 \\
\hline & 31.0 & 57.57 & 60.69 & 98.73 & 103.39 & 104.02 & 104.02 & 103.39 & 98.72 & 60.66 & 57.37 \\
\hline & 32.0 & 57.67 & 60.90 & 98.77 & 103.44 & 104.10 & 104.10 & 103.44 & 98.76 & 60.87 & 57.46 \\
\hline & 33.0 & 57.75 & 61.09 & 98.97 & 103.66 & 104.34 & 104.34 & 103.66 & 98.96 & 61.06 & 57.51 \\
\hline & 34.0 & 57.83 & 61.30 & 98.91 & 103.60 & 104.29 & 104.29 & 103.59 & 98.90 & 61.27 & 57.61 \\
\hline & 35.0 & 57.90 & 61.40 & 97.98 & 102.59 & 103.29 & 103.29 & 102.58 & 97.97 & 61.37 & 57.73 \\
\hline & 36.0 & 57.96 & 61.59 & 97.91 & 102.51 & 103.23 & 103.23 & 102.51 & 97.90 & 61.57 & 57.82 \\
\hline & 37.0 & 57.78 & 59.26 & 63.51 & 65.31 & 66.05 & 66.05 & 65.31 & 63.51 & 59.24 & 57.63 \\
\hline & 37.5 & 57.71 & 58.72 & 60.30 & 61.40 & 62.13 & 62.13 & 61.40 & 60.29 & 58.70 & 57.49 \\
\hline \multirow{9}{*}{ A-6 } & 37.5 & 57.71 & 58.72 & 60.30 & 61.40 & 62.13 & 62.13 & 61.40 & 60.29 & 58.70 & 57.49 \\
\hline & 38.0 & 57.80 & 59.32 & 63.30 & 65.02 & 65.74 & 65.74 & 65.01 & 63.29 & 59.30 & 57.59 \\
\hline & 39.0 & 58.04 & 61.81 & 95.70 & 100.06 & 100.77 & 100.77 & 100.05 & 95.69 & 61.78 & 57.83 \\
\hline & 40.0 & 58.05 & 61.82 & 93.85 & 98.04 & 98.77 & 98.77 & 98.04 & 93.85 & 61.80 & 57.86 \\
\hline & 41.0 & 58.06 & 61.84 & 91.96 & 95.97 & 96.71 & 96.70 & 95.97 & 91.95 & 61.82 & 57.88 \\
\hline & 42.0 & 58.06 & 61.83 & 89.86 & 93.68 & 94.42 & 94.42 & 93.67 & 89.86 & 61.81 & 57.85 \\
\hline & 43.0 & 58.05 & 61.91 & 89.72 & 93.48 & 94.23 & 94.22 & 93.48 & 89.71 & 61.89 & 57.86 \\
\hline & 44.0 & 57.99 & 61.84 & 87.65 & 91.15 & 91.89 & 91.89 & 91.15 & 87.64 & 61.81 & 57.87 \\
\hline & 45.0 & 57.78 & 59.88 & 61.49 & 62.62 & 63.37 & 63.37 & 62.62 & 61.48 & 59.86 & 57.70 \\
\hline
\end{tabular}


Table B - 27: Temperature $\left({ }^{\circ} \mathrm{C}\right)$ map of the east side of position B at MOC2 145A (total 89.7 EFPD).

\begin{tabular}{|c|c|c|c|c|c|c|c|c|c|c|c|}
\hline \multirow{2}{*}{$\begin{array}{c}\text { Plate } \\
\text { Location }\end{array}$} & \multirow{2}{*}{$\begin{array}{c}\text { Axial } \\
\text { Location (in) }\end{array}$} & \multicolumn{10}{|c|}{ Width of Plate (inches) } \\
\hline & & 0.00 & 0.08 & 0.16 & 0.25 & 0.50 & 1.00 & 1.50 & 2.00 & 2.08 & 2.16 \\
\hline \multirow{9}{*}{ B-1 } & 0.0 & 51.67 & 51.79 & 51.76 & 51.77 & 51.77 & 51.77 & 51.77 & 51.76 & 51.79 & 51.67 \\
\hline & 1.0 & 52.98 & 53.94 & 75.82 & 78.22 & 78.29 & 78.29 & 78.22 & 75.82 & 53.91 & 52.88 \\
\hline & 2.0 & 53.21 & 54.29 & 78.83 & 81.48 & 81.57 & 81.57 & 81.48 & 78.83 & 54.24 & 52.95 \\
\hline & 3.0 & 53.42 & 54.51 & 79.33 & 82.06 & 82.17 & 82.17 & 82.06 & 79.33 & 54.44 & 53.05 \\
\hline & 4.0 & 53.68 & 54.84 & 81.70 & 84.63 & 84.77 & 84.77 & 84.63 & 81.70 & 54.78 & 53.33 \\
\hline & 5.0 & 53.91 & 55.17 & 83.96 & 87.07 & 87.23 & 87.23 & 87.07 & 83.95 & 55.10 & 53.49 \\
\hline & 6.0 & 54.18 & 55.58 & 86.75 & 90.09 & 90.27 & 90.27 & 90.09 & 86.74 & 55.50 & 53.68 \\
\hline & 7.0 & 54.17 & 53.42 & 56.14 & 57.06 & 57.24 & 57.24 & 57.06 & 56.14 & 53.33 & 53.64 \\
\hline & 7.5 & 54.18 & 52.94 & 53.25 & 53.52 & 53.68 & 53.68 & 53.52 & 53.24 & 52.84 & 53.63 \\
\hline \multirow{9}{*}{ B-2 } & 7.5 & 54.18 & 52.94 & 53.25 & 53.52 & 53.68 & 53.68 & 53.52 & 53.24 & 52.84 & 53.63 \\
\hline & 8.0 & 54.37 & 53.59 & 56.50 & 57.49 & 57.68 & 57.68 & 57.49 & 56.50 & 53.49 & 53.79 \\
\hline & 9.0 & 54.85 & 56.24 & 89.83 & 93.42 & 93.64 & 93.64 & 93.42 & 89.83 & 56.21 & 54.75 \\
\hline & 10.0 & 55.07 & 56.49 & 90.73 & 94.40 & 94.64 & 94.64 & 94.40 & 90.73 & 56.43 & 54.82 \\
\hline & 11.0 & 55.32 & 56.80 & 91.94 & 95.71 & 95.98 & 95.98 & 95.71 & 91.94 & 56.70 & 54.82 \\
\hline & 12.0 & 55.54 & 57.03 & 92.52 & 96.35 & 96.64 & 96.64 & 96.35 & 92.52 & 56.91 & 54.85 \\
\hline & 13.0 & 55.75 & 57.25 & 92.81 & 96.68 & 96.99 & 96.99 & 96.67 & 92.80 & 57.14 & 55.11 \\
\hline & 14.0 & 55.94 & 57.42 & 93.78 & 97.70 & 98.06 & 98.06 & 97.69 & 93.77 & 57.30 & 55.25 \\
\hline & 15.0 & 55.82 & 54.41 & 55.09 & 55.64 & 55.99 & 55.99 & 55.64 & 55.08 & 54.27 & 55.09 \\
\hline \multirow{9}{*}{ B-3 } & 15.0 & 55.82 & 54.41 & 55.09 & 55.64 & 55.99 & 55.99 & 55.64 & 55.08 & 54.27 & 55.09 \\
\hline & 16.0 & 56.35 & 57.90 & 94.89 & 99.00 & 99.39 & 99.39 & 99.00 & 94.88 & 57.76 & 55.53 \\
\hline & 17.0 & 56.55 & 58.15 & 95.38 & 99.60 & 100.02 & 100.02 & 99.59 & 95.38 & 58.06 & 56.22 \\
\hline & 18.0 & 56.73 & 58.36 & 95.62 & 99.90 & 100.34 & 100.34 & 99.89 & 95.61 & 58.31 & 56.57 \\
\hline & 19.0 & 56.94 & 58.61 & 95.90 & 100.26 & 100.73 & 100.73 & 100.25 & 95.90 & 58.51 & 56.58 \\
\hline & 20.0 & 57.11 & 58.81 & 96.06 & 100.46 & 100.95 & 100.95 & 100.45 & 96.05 & 58.66 & 56.45 \\
\hline & 21.0 & 57.30 & 59.05 & 96.45 & 100.92 & 101.43 & 101.43 & 100.91 & 96.44 & 58.91 & 56.54 \\
\hline & 22.0 & 57.20 & 56.55 & 60.39 & 61.92 & 62.44 & 62.44 & 61.91 & 60.38 & 56.41 & 56.41 \\
\hline & 22.5 & 57.17 & 55.96 & 56.89 & 57.66 & 58.16 & 58.16 & 57.66 & 56.88 & 55.90 & 57.05 \\
\hline \multirow{9}{*}{ B-4 } & 22.5 & 57.17 & 55.96 & 56.89 & 57.66 & 58.16 & 58.16 & 57.66 & 56.88 & 55.90 & 57.05 \\
\hline & 23.0 & 57.33 & 56.70 & 60.52 & 62.05 & 62.57 & 62.57 & 62.05 & 60.51 & 56.56 & 56.54 \\
\hline & 24.0 & 57.74 & 59.61 & 97.43 & 101.97 & 102.51 & 102.51 & 101.96 & 97.42 & 59.45 & 56.84 \\
\hline & 25.0 & 57.87 & 59.77 & 97.03 & 101.55 & 102.11 & 102.11 & 101.54 & 97.02 & 59.62 & 57.17 \\
\hline & 26.0 & 58.01 & 60.03 & 97.42 & 101.98 & 102.56 & 102.56 & 101.97 & 97.41 & 59.92 & 57.68 \\
\hline & 27.0 & 58.13 & 60.22 & 97.46 & 102.03 & 102.63 & 102.63 & 102.02 & 97.45 & 60.17 & 57.94 \\
\hline & 28.0 & 58.24 & 60.43 & 97.69 & 102.29 & 102.91 & 102.90 & 102.28 & 97.68 & 60.33 & 57.87 \\
\hline & 29.0 & 58.30 & 60.56 & 98.03 & 102.61 & 103.23 & 103.23 & 102.60 & 98.02 & 60.41 & 57.61 \\
\hline & 30.0 & 58.09 & 57.57 & 58.79 & 59.75 & 60.39 & 60.38 & 59.75 & 58.77 & 57.41 & 57.27 \\
\hline \multirow{9}{*}{ B-5 } & 30.0 & 58.09 & 57.57 & 58.79 & 59.76 & 60.39 & 60.38 & 59.75 & 58.77 & 57.41 & 57.27 \\
\hline & 31.0 & 58.47 & 60.80 & 96.43 & 100.87 & 101.51 & 101.51 & 100.87 & 96.42 & 60.67 & 57.78 \\
\hline & 32.0 & 58.56 & 60.98 & 96.10 & 100.53 & 101.18 & 101.18 & 100.52 & 96.09 & 60.83 & 57.79 \\
\hline & 33.0 & 58.62 & 61.16 & 96.27 & 100.71 & 101.38 & 101.37 & 100.70 & 96.26 & 61.01 & 57.77 \\
\hline & 34.0 & 58.68 & 61.36 & 96.13 & 100.55 & 101.24 & 101.24 & 100.55 & 96.11 & 61.21 & 58.00 \\
\hline & 35.0 & 58.72 & 61.51 & 95.76 & 100.15 & 100.85 & 100.85 & 100.15 & 95.75 & 61.40 & 58.35 \\
\hline & 36.0 & 58.75 & 61.66 & 95.24 & 99.59 & 100.30 & 100.30 & 99.58 & 95.23 & 61.59 & 58.48 \\
\hline & 37.0 & 58.55 & 59.52 & 63.41 & 65.12 & 65.85 & 65.85 & 65.11 & 63.38 & 59.42 & 58.27 \\
\hline & 37.5 & 58.47 & 59.03 & 60.45 & 61.53 & 62.24 & 62.24 & 61.52 & 60.42 & 58.89 & 57.75 \\
\hline \multirow{9}{*}{ B-6 } & 37.5 & 58.47 & 59.03 & 60.45 & 61.53 & 62.24 & 62.24 & 61.52 & 60.43 & 58.89 & 57.75 \\
\hline & 38.0 & 58.55 & 59.61 & 63.34 & 65.02 & 65.73 & 65.73 & 65.01 & 63.32 & 59.47 & 57.94 \\
\hline & 39.0 & 58.75 & 62.00 & 94.60 & 98.84 & 99.55 & 99.55 & 98.83 & 94.58 & 61.87 & 58.08 \\
\hline & 40.0 & 58.74 & 62.01 & 92.77 & 96.84 & 97.57 & 97.56 & 96.84 & 92.75 & 61.89 & 58.08 \\
\hline & 41.0 & 58.71 & 62.02 & 90.83 & 94.72 & 95.44 & 95.44 & 94.71 & 90.81 & 61.90 & 58.12 \\
\hline & 42.0 & 58.67 & 62.03 & 89.12 & 92.82 & 93.55 & 93.55 & 92.81 & 89.11 & 61.91 & 58.01 \\
\hline & 43.0 & 58.63 & 62.12 & 89.02 & 92.68 & 93.42 & 93.42 & 92.67 & 89.01 & 62.00 & 58.12 \\
\hline & 44.0 & 58.54 & 62.06 & 87.25 & 90.68 & 91.41 & 91.41 & 90.67 & 87.24 & 61.98 & 58.29 \\
\hline & 45.0 & 58.31 & 60.15 & 61.68 & 62.78 & 63.52 & 63.52 & 62.77 & 61.65 & 60.10 & 58.18 \\
\hline
\end{tabular}


Table B - 28: Temperature $\left({ }^{\circ} \mathrm{C}\right)$ map of the west side of position B at MOC2 145A (total 89.7 EFPD).

\begin{tabular}{|c|c|c|c|c|c|c|c|c|c|c|c|}
\hline \multirow{2}{*}{$\begin{array}{c}\text { Plate } \\
\text { Location }\end{array}$} & \multirow{2}{*}{$\begin{array}{c}\text { Axial } \\
\text { Location (in) }\end{array}$} & \multicolumn{10}{|c|}{ Width of Plate (inches) } \\
\hline & & 0.00 & 0.08 & 0.16 & 0.25 & 0.50 & 1.00 & 1.50 & 2.00 & 2.08 & 2.16 \\
\hline \multirow{9}{*}{ B-1 } & 0.0 & 51.67 & 51.79 & 51.76 & 51.77 & 51.77 & 51.77 & 51.77 & 51.76 & 51.79 & 51.67 \\
\hline & 1.0 & 53.29 & 53.92 & 75.81 & 78.20 & 78.27 & 78.27 & 78.20 & 75.81 & 53.90 & 53.17 \\
\hline & 2.0 & 53.55 & 54.25 & 78.81 & 81.44 & 81.53 & 81.53 & 81.44 & 78.80 & 54.22 & 53.11 \\
\hline & 3.0 & 53.80 & 54.45 & 79.28 & 81.99 & 82.10 & 82.10 & 81.99 & 79.27 & 54.41 & 53.07 \\
\hline & 4.0 & 54.09 & 54.77 & 81.61 & 84.52 & 84.64 & 84.64 & 84.51 & 81.61 & 54.73 & 53.40 \\
\hline & 5.0 & 54.35 & 55.07 & 83.83 & 86.91 & 87.05 & 87.05 & 86.91 & 83.83 & 55.03 & 53.51 \\
\hline & 6.0 & 54.64 & 55.46 & 86.58 & 89.88 & 90.03 & 90.03 & 89.88 & 86.57 & 55.40 & 53.71 \\
\hline & 7.0 & 54.69 & 53.27 & 55.97 & 56.84 & 56.99 & 56.99 & 56.84 & 55.96 & 53.21 & 53.76 \\
\hline & 7.5 & 54.74 & 52.78 & 53.06 & 53.28 & 53.41 & 53.41 & 53.28 & 53.05 & 52.71 & 53.78 \\
\hline \multirow{9}{*}{ B-2 } & 7.5 & 54.74 & 52.78 & 53.06 & 53.28 & 53.41 & 53.41 & 53.28 & 53.05 & 52.71 & 53.78 \\
\hline & 8.0 & 54.92 & 53.42 & 56.31 & 57.24 & 57.40 & 57.40 & 57.24 & 56.30 & 53.35 & 53.91 \\
\hline & 9.0 & 55.38 & 56.06 & 89.57 & 93.10 & 93.27 & 93.27 & 93.10 & 89.57 & 56.03 & 55.42 \\
\hline & 10.0 & 55.63 & 56.29 & 90.43 & 94.03 & 94.22 & 94.22 & 94.02 & 90.42 & 56.24 & 55.35 \\
\hline & 11.0 & 55.90 & 56.57 & 91.58 & 95.27 & 95.48 & 95.48 & 95.27 & 91.58 & 56.49 & 55.05 \\
\hline & 12.0 & 56.14 & 56.76 & 92.11 & 95.85 & 96.07 & 96.07 & 95.84 & 92.11 & 56.67 & 54.82 \\
\hline & 13.0 & 56.36 & 56.95 & 92.35 & 96.11 & 96.34 & 96.34 & 96.11 & 92.34 & 56.87 & 55.11 \\
\hline & 14.0 & 56.56 & 57.08 & 93.26 & 97.06 & 97.30 & 97.30 & 97.05 & 93.26 & 56.99 & 55.21 \\
\hline & 15.0 & 56.51 & 54.03 & 54.59 & 55.03 & 55.30 & 55.30 & 55.03 & 54.58 & 53.93 & 55.21 \\
\hline \multirow{9}{*}{ B-3 } & 15.0 & 56.51 & 54.03 & 54.59 & 55.03 & 55.30 & 55.30 & 55.03 & 54.58 & 53.93 & 55.21 \\
\hline & 16.0 & 57.01 & 57.52 & 94.30 & 98.20 & 98.46 & 98.46 & 98.20 & 94.29 & 57.41 & 55.59 \\
\hline & 17.0 & 57.20 & 57.74 & 94.77 & 98.73 & 99.04 & 99.04 & 98.72 & 94.76 & 57.67 & 56.83 \\
\hline & 18.0 & 57.40 & 57.92 & 94.96 & 98.98 & 99.30 & 99.30 & 98.97 & 94.95 & 57.87 & 57.37 \\
\hline & 19.0 & 57.61 & 58.13 & 95.20 & 99.27 & 99.61 & 99.61 & 99.26 & 95.18 & 58.06 & 57.17 \\
\hline & 20.0 & 57.77 & 58.30 & 95.30 & 99.41 & 99.77 & 99.76 & 99.40 & 95.29 & 58.19 & 56.66 \\
\hline & 21.0 & 57.95 & 58.51 & 95.64 & 99.80 & 100.17 & 100.17 & 99.79 & 95.62 & 58.39 & 56.58 \\
\hline & 22.0 & 57.89 & 55.97 & 59.64 & 61.01 & 61.41 & 61.41 & 61.00 & 59.62 & 55.86 & 56.36 \\
\hline & 22.5 & 57.87 & 55.36 & 56.11 & 56.72 & 57.10 & 57.10 & 56.71 & 56.09 & 55.31 & 58.00 \\
\hline \multirow{9}{*}{ B-4 } & 22.5 & 57.87 & 55.36 & 56.11 & 56.72 & 57.10 & 57.10 & 56.71 & 56.09 & 55.31 & 57.99 \\
\hline & 23.0 & 58.02 & 56.10 & 59.74 & 61.11 & 61.52 & 61.51 & 61.11 & 59.72 & 55.99 & 56.47 \\
\hline & 24.0 & 58.38 & 58.99 & 96.45 & 100.72 & 101.10 & 101.10 & 100.71 & 96.43 & 58.86 & 56.71 \\
\hline & 25.0 & 58.49 & 59.13 & 96.01 & 100.24 & 100.64 & 100.64 & 100.23 & 95.99 & 59.00 & 57.33 \\
\hline & 26.0 & 58.61 & 59.34 & 96.31 & 100.60 & 101.02 & 101.01 & 100.59 & 96.29 & 59.25 & 58.29 \\
\hline & 27.0 & 58.72 & 59.50 & 96.28 & 100.58 & 101.01 & 101.01 & 100.57 & 96.26 & 59.43 & 58.67 \\
\hline & 28.0 & 58.81 & 59.68 & 96.44 & 100.77 & 101.21 & 101.21 & 100.76 & 96.42 & 59.58 & 58.38 \\
\hline & 29.0 & 58.85 & 59.76 & 96.69 & 101.02 & 101.46 & 101.45 & 101.01 & 96.66 & 59.63 & 57.69 \\
\hline & 30.0 & 58.66 & 56.73 & 57.70 & 58.45 & 58.93 & 58.92 & 58.44 & 57.67 & 56.59 & 57.18 \\
\hline \multirow{9}{*}{ B-5 } & 30.0 & 58.66 & 56.73 & 57.70 & 58.45 & 58.93 & 58.92 & 58.44 & 57.67 & 56.59 & 57.18 \\
\hline & 31.0 & 58.97 & 59.94 & 95.07 & 99.19 & 99.63 & 99.62 & 99.17 & 95.04 & 59.82 & 57.69 \\
\hline & 32.0 & 59.02 & 60.09 & 94.69 & 98.78 & 99.24 & 99.24 & 98.77 & 94.67 & 59.97 & 57.55 \\
\hline & 33.0 & 59.06 & 60.24 & 94.79 & 98.90 & 99.37 & 99.37 & 98.89 & 94.77 & 60.10 & 57.48 \\
\hline & 34.0 & 59.08 & 60.39 & 94.58 & 98.67 & 99.15 & 99.15 & 98.66 & 94.55 & 60.26 & 57.95 \\
\hline & 35.0 & 59.09 & 60.50 & 94.16 & 98.20 & 98.69 & 98.68 & 98.18 & 94.13 & 60.40 & 58.67 \\
\hline & 36.0 & 59.08 & 60.61 & 93.58 & 97.55 & 98.04 & 98.04 & 97.53 & 93.55 & 60.52 & 58.81 \\
\hline & 37.0 & 58.88 & 58.43 & 62.02 & 63.50 & 64.04 & 64.04 & 63.48 & 61.98 & 58.32 & 58.65 \\
\hline & 37.5 & 58.80 & 57.92 & 59.03 & 59.86 & 60.39 & 60.38 & 59.84 & 58.99 & 57.78 & 57.51 \\
\hline \multirow{9}{*}{ B-6 } & 37.5 & 58.80 & 57.92 & 59.03 & 59.86 & 60.39 & 60.38 & 59.84 & 58.99 & 57.78 & 57.51 \\
\hline & 38.0 & 58.84 & 58.48 & 61.93 & 63.36 & 63.89 & 63.89 & 63.34 & 61.89 & 58.35 & 57.83 \\
\hline & 39.0 & 58.97 & 60.86 & 92.82 & 96.66 & 97.14 & 97.14 & 96.64 & 92.79 & 60.73 & 57.77 \\
\hline & 40.0 & 58.92 & 60.83 & 90.99 & 94.60 & 95.10 & 95.09 & 94.58 & 90.96 & 60.71 & 57.65 \\
\hline & 41.0 & 58.84 & 60.80 & 89.02 & 92.46 & 92.92 & 92.92 & 92.45 & 88.99 & 60.68 & 57.76 \\
\hline & 42.0 & 58.77 & 60.78 & 87.25 & 90.55 & 91.02 & 91.01 & 90.53 & 87.22 & 60.65 & 57.54 \\
\hline & 43.0 & 58.69 & 60.83 & 87.10 & 90.36 & 90.83 & 90.82 & 90.34 & 87.07 & 60.71 & 57.84 \\
\hline & 44.0 & 58.55 & 60.73 & 85.26 & 88.33 & 88.79 & 88.79 & 88.31 & 85.22 & 60.64 & 58.22 \\
\hline & 45.0 & 58.32 & 58.79 & 59.99 & 60.83 & 61.38 & 61.37 & 60.81 & 59.95 & 58.72 & 58.19 \\
\hline
\end{tabular}


Table B - 29: Temperature $\left({ }^{\circ} \mathrm{C}\right)$ map of the east side of position A at EOC 145A (total 106.4 EFPD).

\begin{tabular}{|c|c|c|c|c|c|c|c|c|c|c|c|}
\hline \multirow{2}{*}{$\begin{array}{c}\text { Plate } \\
\text { Location }\end{array}$} & \multirow{2}{*}{$\begin{array}{c}\text { Axial } \\
\text { Location (in) }\end{array}$} & \multicolumn{10}{|c|}{ Width of Plate (inches) } \\
\hline & & 0.00 & 0.08 & 0.16 & 0.25 & 0.50 & 1.00 & 1.50 & 2.00 & 2.08 & 2.16 \\
\hline \multirow{9}{*}{ A-1 } & 0.0 & 51.67 & 51.77 & 51.76 & 51.77 & 51.77 & 51.77 & 51.77 & 51.76 & 51.77 & 51.67 \\
\hline & 1.0 & 52.51 & 53.86 & 75.65 & 78.04 & 78.11 & 78.11 & 78.04 & 75.65 & 53.85 & 52.49 \\
\hline & 2.0 & 52.67 & 54.16 & 78.40 & 81.01 & 81.10 & 81.10 & 81.01 & 78.40 & 54.16 & 52.64 \\
\hline & 3.0 & 52.83 & 54.34 & 78.84 & 81.53 & 81.63 & 81.63 & 81.53 & 78.84 & 54.34 & 52.79 \\
\hline & 4.0 & 53.02 & 54.63 & 81.05 & 83.91 & 84.03 & 84.03 & 83.91 & 81.05 & 54.63 & 52.99 \\
\hline & 5.0 & 53.19 & 54.93 & 83.35 & 86.40 & 86.54 & 86.54 & 86.40 & 83.35 & 54.92 & 53.15 \\
\hline & 6.0 & 53.39 & 55.22 & 85.21 & 88.41 & 88.56 & 88.56 & 88.41 & 85.21 & 55.21 & 53.33 \\
\hline & 7.0 & 53.38 & 53.11 & 55.77 & 56.62 & 56.77 & 56.77 & 56.62 & 55.77 & 53.09 & 53.30 \\
\hline & 7.5 & 53.39 & 52.63 & 52.98 & 53.20 & 53.33 & 53.33 & 53.20 & 52.98 & 52.61 & 53.31 \\
\hline \multirow{9}{*}{ A-2 } & 7.5 & 53.39 & 52.63 & 52.98 & 53.20 & 53.33 & 53.33 & 53.20 & 52.98 & 52.61 & 53.31 \\
\hline & 8.0 & 53.53 & 53.28 & 56.29 & 57.24 & 57.40 & 57.40 & 57.24 & 56.29 & 53.26 & 53.45 \\
\hline & 9.0 & 53.90 & 55.95 & 90.14 & 93.72 & 93.89 & 93.89 & 93.72 & 90.14 & 55.94 & 53.87 \\
\hline & 10.0 & 54.07 & 56.15 & 90.82 & 94.46 & 94.66 & 94.66 & 94.46 & 90.82 & 56.14 & 54.02 \\
\hline & 11.0 & 54.26 & 56.33 & 91.14 & 94.81 & 95.02 & 95.02 & 94.81 & 91.14 & 56.31 & 54.19 \\
\hline & 12.0 & 54.43 & 56.53 & 91.97 & 95.71 & 95.93 & 95.93 & 95.71 & 91.97 & 56.51 & 54.35 \\
\hline & 13.0 & 54.60 & 56.70 & 92.18 & 95.95 & 96.18 & 96.18 & 95.95 & 92.18 & 56.68 & 54.53 \\
\hline & 14.0 & 54.74 & 56.77 & 92.66 & 96.43 & 96.67 & 96.67 & 96.43 & 92.66 & 56.75 & 54.67 \\
\hline & 15.0 & 54.66 & 53.74 & 54.38 & 54.83 & 55.09 & 55.09 & 54.83 & 54.38 & 53.71 & 54.56 \\
\hline \multirow{9}{*}{ A-3 } & 15.0 & 54.66 & 53.74 & 54.38 & 54.83 & 55.09 & 55.09 & 54.83 & 54.38 & 53.71 & 54.56 \\
\hline & 16.0 & 55.05 & 56.68 & 88.32 & 91.79 & 92.05 & 92.05 & 91.79 & 88.32 & 56.66 & 54.93 \\
\hline & 17.0 & 55.21 & 56.86 & 88.65 & 92.15 & 92.42 & 92.42 & 92.15 & 88.65 & 56.85 & 55.14 \\
\hline & 18.0 & 55.36 & 57.02 & 88.81 & 92.33 & 92.61 & 92.61 & 92.33 & 88.81 & 57.01 & 55.31 \\
\hline & 19.0 & 55.53 & 57.20 & 89.09 & 92.64 & 92.93 & 92.93 & 92.64 & 89.09 & 57.18 & 55.46 \\
\hline & 20.0 & 55.67 & 57.32 & 88.95 & 92.49 & 92.79 & 92.79 & 92.49 & 88.95 & 57.30 & 55.56 \\
\hline & 21.0 & 55.82 & 57.47 & 88.94 & 92.48 & 92.79 & 92.79 & 92.48 & 88.94 & 57.45 & 55.72 \\
\hline & 22.0 & 55.77 & 55.34 & 58.52 & 59.74 & 60.13 & 60.13 & 59.74 & 58.52 & 55.32 & 55.67 \\
\hline & 22.5 & 55.75 & 54.84 & 55.59 & 56.17 & 56.53 & 56.53 & 56.17 & 55.59 & 54.82 & 55.70 \\
\hline \multirow{9}{*}{ A-4 } & 22.5 & 55.75 & 54.84 & 55.59 & 56.17 & 56.53 & 56.53 & 56.17 & 55.59 & 54.82 & 55.70 \\
\hline & 23.0 & 55.88 & 55.51 & 58.95 & 60.24 & 60.62 & 60.62 & 60.24 & 58.94 & 55.49 & 55.79 \\
\hline & 24.0 & 56.20 & 58.19 & 93.08 & 96.92 & 97.26 & 97.26 & 96.92 & 93.08 & 58.16 & 56.09 \\
\hline & 25.0 & 56.32 & 58.36 & 93.32 & 97.20 & 97.57 & 97.57 & 97.20 & 93.32 & 58.33 & 56.21 \\
\hline & 26.0 & 56.45 & 58.53 & 93.45 & 97.34 & 97.75 & 97.75 & 97.34 & 93.44 & 58.51 & 56.38 \\
\hline & 27.0 & 56.56 & 58.69 & 93.75 & 97.71 & 98.12 & 98.12 & 97.71 & 93.75 & 58.68 & 56.50 \\
\hline & 28.0 & 56.66 & 58.84 & 93.88 & 97.86 & 98.29 & 98.29 & 97.86 & 93.88 & 58.82 & 56.58 \\
\hline & 29.0 & 56.72 & 58.87 & 93.63 & 97.54 & 97.97 & 97.97 & 97.54 & 93.63 & 58.84 & 56.61 \\
\hline & 30.0 & 56.58 & 56.01 & 56.99 & 57.72 & 58.17 & 58.17 & 57.72 & 56.99 & 55.98 & 56.47 \\
\hline \multirow{9}{*}{ A-5 } & 30.0 & 56.58 & 56.01 & 56.99 & 57.72 & 58.17 & 58.17 & 57.72 & 56.99 & 55.98 & 56.47 \\
\hline & 31.0 & 56.89 & 59.20 & 94.15 & 98.15 & 98.58 & 98.58 & 98.15 & 94.15 & 59.18 & 56.80 \\
\hline & 32.0 & 56.98 & 59.37 & 94.27 & 98.31 & 98.75 & 98.75 & 98.31 & 94.27 & 59.35 & 56.89 \\
\hline & 33.0 & 57.05 & 59.51 & 94.40 & 98.47 & 98.93 & 98.93 & 98.47 & 94.40 & 59.49 & 56.94 \\
\hline & 34.0 & 57.12 & 59.66 & 94.35 & 98.42 & 98.89 & 98.89 & 98.42 & 94.35 & 59.64 & 57.02 \\
\hline & 35.0 & 57.18 & 59.75 & 93.89 & 97.91 & 98.39 & 98.39 & 97.91 & 93.88 & 59.74 & 57.10 \\
\hline & 36.0 & 57.23 & 59.87 & 93.61 & 97.59 & 98.08 & 98.08 & 97.59 & 93.60 & 59.86 & 57.17 \\
\hline & 37.0 & 57.09 & 57.62 & 61.30 & 62.79 & 63.32 & 63.32 & 62.78 & 61.30 & 57.60 & 57.02 \\
\hline & 37.5 & 57.03 & 57.09 & 58.22 & 59.04 & 59.55 & 59.55 & 59.03 & 58.21 & 57.06 & 56.93 \\
\hline \multirow{9}{*}{ A-6 } & 37.5 & 57.03 & 57.09 & 58.22 & 59.04 & 59.56 & 59.55 & 59.03 & 58.22 & 57.06 & 56.93 \\
\hline & 38.0 & 57.11 & 57.64 & 61.09 & 62.50 & 63.03 & 63.03 & 62.50 & 61.09 & 57.62 & 57.01 \\
\hline & 39.0 & 57.29 & 59.99 & 91.64 & 95.36 & 95.83 & 95.83 & 95.36 & 91.64 & 59.97 & 57.20 \\
\hline & 40.0 & 57.30 & 60.00 & 90.30 & 93.91 & 94.36 & 94.36 & 93.91 & 90.30 & 59.98 & 57.23 \\
\hline & 41.0 & 57.31 & 59.99 & 88.70 & 92.18 & 92.64 & 92.64 & 92.18 & 88.70 & 59.98 & 57.24 \\
\hline & 42.0 & 57.31 & 59.95 & 86.75 & 90.07 & 90.54 & 90.54 & 90.07 & 86.75 & 59.93 & 57.22 \\
\hline & 43.0 & 57.30 & 59.99 & 86.57 & 89.85 & 90.31 & 90.31 & 89.85 & 86.57 & 59.97 & 57.22 \\
\hline & 44.0 & 57.25 & 59.87 & 84.52 & 87.60 & 88.06 & 88.06 & 87.60 & 84.52 & 59.86 & 57.20 \\
\hline & 45.0 & 57.07 & 57.91 & 59.10 & 59.94 & 60.48 & 60.48 & 59.94 & 59.10 & 57.90 & 57.04 \\
\hline
\end{tabular}


Table B - 30: Temperature $\left({ }^{\circ} \mathrm{C}\right)$ map of the west side of position A at EOC 145A (total 106.4 EFPD).

\begin{tabular}{|c|c|c|c|c|c|c|c|c|c|c|c|}
\hline \multirow{2}{*}{$\begin{array}{c}\text { Plate } \\
\text { Location }\end{array}$} & \multirow{2}{*}{$\begin{array}{c}\text { Axial } \\
\text { Location (in) }\end{array}$} & \multicolumn{10}{|c|}{ Width of Plate (inches) } \\
\hline & & 0.00 & 0.08 & 0.16 & 0.25 & 0.50 & 1.00 & 1.50 & 2.00 & 2.08 & 2.16 \\
\hline \multirow{9}{*}{ A-1 } & 0.0 & 51.67 & 51.77 & 51.76 & 51.77 & 51.77 & 51.77 & 51.77 & 51.76 & 51.77 & 51.67 \\
\hline & 1.0 & 52.63 & 53.85 & 75.66 & 78.05 & 78.12 & 78.12 & 78.05 & 75.66 & 53.85 & 52.59 \\
\hline & 2.0 & 52.81 & 54.17 & 78.43 & 81.05 & 81.14 & 81.14 & 81.05 & 78.43 & 54.17 & 52.74 \\
\hline & 3.0 & 52.99 & 54.37 & 78.90 & 81.60 & 81.71 & 81.71 & 81.60 & 78.90 & 54.36 & 52.90 \\
\hline & 4.0 & 53.20 & 54.68 & 81.15 & 84.03 & 84.17 & 84.17 & 84.03 & 81.15 & 54.67 & 53.12 \\
\hline & 5.0 & 53.39 & 55.00 & 83.49 & 86.57 & 86.73 & 86.73 & 86.57 & 83.49 & 54.99 & 53.30 \\
\hline & 6.0 & 53.60 & 55.32 & 85.39 & 88.64 & 88.81 & 88.81 & 88.64 & 85.39 & 55.31 & 53.48 \\
\hline & 7.0 & 53.57 & 53.23 & 55.96 & 56.85 & 57.02 & 57.02 & 56.85 & 55.96 & 53.22 & 53.43 \\
\hline & 7.5 & 53.57 & 52.76 & 53.19 & 53.45 & 53.61 & 53.61 & 53.45 & 53.19 & 52.75 & 53.42 \\
\hline \multirow{9}{*}{ A-2 } & 7.5 & 53.57 & 52.76 & 53.19 & 53.45 & 53.61 & 53.61 & 53.45 & 53.19 & 52.75 & 53.42 \\
\hline & 8.0 & 53.73 & 53.42 & 56.51 & 57.50 & 57.69 & 57.69 & 57.50 & 56.50 & 53.41 & 53.57 \\
\hline & 9.0 & 54.17 & 56.11 & 90.43 & 94.06 & 94.28 & 94.28 & 94.06 & 90.43 & 56.10 & 54.10 \\
\hline & 10.0 & 54.35 & 56.33 & 91.15 & 94.86 & 95.09 & 95.09 & 94.86 & 91.15 & 56.32 & 54.25 \\
\hline & 11.0 & 54.56 & 56.55 & 91.52 & 95.27 & 95.53 & 95.53 & 95.27 & 91.52 & 56.54 & 54.41 \\
\hline & 12.0 & 54.75 & 56.78 & 92.40 & 96.22 & 96.51 & 96.51 & 96.22 & 92.40 & 56.77 & 54.57 \\
\hline & 13.0 & 54.93 & 56.98 & 92.67 & 96.53 & 96.83 & 96.83 & 96.52 & 92.67 & 56.97 & 54.78 \\
\hline & 14.0 & 55.08 & 57.09 & 93.21 & 97.07 & 97.39 & 97.39 & 97.07 & 93.21 & 57.08 & 54.92 \\
\hline & 15.0 & 54.96 & 54.09 & 54.90 & 55.44 & 55.78 & 55.78 & 55.44 & 54.90 & 54.08 & 54.76 \\
\hline \multirow{9}{*}{ A-3 } & 15.0 & 54.96 & 54.09 & 54.90 & 55.44 & 55.78 & 55.78 & 55.44 & 54.90 & 54.08 & 54.76 \\
\hline & 16.0 & 55.42 & 57.06 & 88.96 & 92.54 & 92.88 & 92.88 & 92.54 & 88.96 & 57.04 & 55.18 \\
\hline & 17.0 & 55.60 & 57.27 & 89.33 & 92.95 & 93.31 & 93.31 & 92.95 & 89.33 & 57.26 & 55.46 \\
\hline & 18.0 & 55.76 & 57.46 & 89.54 & 93.19 & 93.56 & 93.56 & 93.19 & 89.54 & 57.45 & 55.66 \\
\hline & 19.0 & 55.94 & 57.69 & 89.89 & 93.57 & 93.96 & 93.96 & 93.56 & 89.88 & 57.67 & 55.80 \\
\hline & 20.0 & 56.09 & 57.85 & 89.80 & 93.49 & 93.90 & 93.90 & 93.48 & 89.80 & 57.83 & 55.88 \\
\hline & 21.0 & 56.25 & 58.04 & 89.86 & 93.55 & 93.98 & 93.98 & 93.55 & 89.86 & 58.02 & 56.05 \\
\hline & 22.0 & 56.17 & 55.95 & 59.35 & 60.71 & 61.20 & 61.20 & 60.71 & 59.35 & 55.94 & 55.98 \\
\hline & 22.5 & 56.15 & 55.46 & 56.45 & 57.17 & 57.63 & 57.63 & 57.17 & 56.44 & 55.45 & 56.05 \\
\hline \multirow{9}{*}{ A-4 } & 22.5 & 56.15 & 55.46 & 56.45 & 57.17 & 57.63 & 57.63 & 57.17 & 56.44 & 55.45 & 56.05 \\
\hline & 23.0 & 56.30 & 56.15 & 59.81 & 61.24 & 61.73 & 61.73 & 61.24 & 59.80 & 56.13 & 56.10 \\
\hline & 24.0 & 56.67 & 58.86 & 94.10 & 98.24 & 98.74 & 98.74 & 98.24 & 94.10 & 58.83 & 56.43 \\
\hline & 25.0 & 56.80 & 59.06 & 94.40 & 98.60 & 99.12 & 99.12 & 98.59 & 94.39 & 59.04 & 56.58 \\
\hline & 26.0 & 56.94 & 59.28 & 94.58 & 98.83 & 99.38 & 99.38 & 98.83 & 94.57 & 59.26 & 56.79 \\
\hline & 27.0 & 57.05 & 59.49 & 94.94 & 99.26 & 99.83 & 99.83 & 99.26 & 94.94 & 59.47 & 56.93 \\
\hline & 28.0 & 57.16 & 59.68 & 95.14 & 99.49 & 100.08 & 100.07 & 99.49 & 95.14 & 59.65 & 57.00 \\
\hline & 29.0 & 57.22 & 59.75 & 94.96 & 99.25 & 99.84 & 99.84 & 99.25 & 94.95 & 59.72 & 57.00 \\
\hline & 30.0 & 57.04 & 56.94 & 58.19 & 59.10 & 59.69 & 59.69 & 59.10 & 58.19 & 56.91 & 56.81 \\
\hline \multirow{9}{*}{ A-5 } & 30.0 & 57.04 & 56.94 & 58.19 & 59.10 & 59.69 & 59.69 & 59.10 & 58.19 & 56.91 & 56.81 \\
\hline & 31.0 & 57.41 & 60.15 & 95.59 & 99.95 & 100.54 & 100.54 & 99.94 & 95.59 & 60.13 & 57.22 \\
\hline & 32.0 & 57.51 & 60.35 & 95.77 & 100.16 & 100.77 & 100.77 & 100.15 & 95.77 & 60.33 & 57.30 \\
\hline & 33.0 & 57.58 & 60.54 & 95.98 & 100.38 & 101.01 & 101.01 & 100.38 & 95.97 & 60.51 & 57.35 \\
\hline & 34.0 & 57.66 & 60.73 & 96.00 & 100.40 & 101.05 & 101.05 & 100.40 & 95.99 & 60.71 & 57.43 \\
\hline & 35.0 & 57.71 & 60.87 & 95.59 & 99.96 & 100.62 & 100.62 & 99.95 & 95.58 & 60.84 & 57.55 \\
\hline & 36.0 & 57.77 & 61.03 & 95.37 & 99.72 & 100.39 & 100.39 & 99.72 & 95.37 & 61.01 & 57.63 \\
\hline & 37.0 & 57.59 & 58.82 & 62.80 & 64.48 & 65.17 & 65.17 & 64.48 & 62.79 & 58.80 & 57.45 \\
\hline & 37.5 & 57.52 & 58.31 & 59.75 & 60.77 & 61.44 & 61.44 & 60.77 & 59.74 & 58.29 & 57.31 \\
\hline \multirow{9}{*}{ A-6 } & 37.5 & 57.52 & 58.31 & 59.75 & 60.77 & 61.44 & 61.44 & 60.77 & 59.74 & 58.29 & 57.31 \\
\hline & 38.0 & 57.61 & 58.88 & 62.61 & 64.23 & 64.90 & 64.90 & 64.22 & 62.61 & 58.85 & 57.41 \\
\hline & 39.0 & 57.83 & 61.25 & 93.49 & 97.63 & 98.30 & 98.29 & 97.62 & 93.49 & 61.23 & 57.63 \\
\hline & 40.0 & 57.84 & 61.30 & 92.17 & 96.19 & 96.87 & 96.87 & 96.19 & 92.16 & 61.27 & 57.66 \\
\hline & 41.0 & 57.85 & 61.33 & 90.63 & 94.48 & 95.17 & 95.16 & 94.48 & 90.63 & 61.31 & 57.67 \\
\hline & 42.0 & 57.84 & 61.33 & 88.75 & 92.39 & 93.08 & 93.08 & 92.38 & 88.75 & 61.30 & 57.64 \\
\hline & 43.0 & 57.83 & 61.41 & 88.62 & 92.21 & 92.91 & 92.91 & 92.21 & 88.61 & 61.38 & 57.65 \\
\hline & 44.0 & 57.77 & 61.33 & 86.65 & 89.99 & 90.68 & 90.68 & 89.98 & 86.64 & 61.31 & 57.66 \\
\hline & 45.0 & 57.56 & 59.40 & 60.91 & 61.96 & 62.66 & 62.66 & 61.96 & 60.90 & 59.38 & 57.49 \\
\hline
\end{tabular}


Table B - 31: Temperature $\left({ }^{\circ} \mathrm{C}\right)$ map of the east side of position B at EOC 145A (total 106.4 EFPD).

\begin{tabular}{|c|c|c|c|c|c|c|c|c|c|c|c|}
\hline \multirow{2}{*}{$\begin{array}{c}\text { Plate } \\
\text { Location }\end{array}$} & \multirow{2}{*}{$\begin{array}{c}\text { Axial } \\
\text { Location (in) }\end{array}$} & \multicolumn{10}{|c|}{ Width of Plate (inches) } \\
\hline & & 0.00 & 0.08 & 0.16 & 0.25 & 0.50 & 1.00 & 1.50 & 2.00 & 2.08 & 2.16 \\
\hline \multirow{9}{*}{ B-1 } & 0.0 & 51.67 & 51.79 & 51.76 & 51.77 & 51.77 & 51.77 & 51.77 & 51.76 & 51.79 & 51.67 \\
\hline & 1.0 & 52.98 & 53.91 & 75.40 & 77.77 & 77.84 & 77.84 & 77.77 & 75.40 & 53.88 & 52.87 \\
\hline & 2.0 & 53.20 & 54.24 & 78.29 & 80.89 & 80.99 & 80.99 & 80.89 & 78.29 & 54.19 & 52.95 \\
\hline & 3.0 & 53.42 & 54.45 & 78.76 & 81.44 & 81.55 & 81.55 & 81.44 & 78.76 & 54.39 & 53.04 \\
\hline & 4.0 & 53.67 & 54.76 & 80.84 & 83.70 & 83.83 & 83.83 & 83.70 & 80.84 & 54.70 & 53.32 \\
\hline & 5.0 & 53.90 & 55.07 & 82.98 & 86.01 & 86.17 & 86.17 & 86.01 & 82.98 & 55.00 & 53.48 \\
\hline & 6.0 & 54.16 & 55.44 & 85.41 & 88.64 & 88.82 & 88.82 & 88.64 & 85.40 & 55.36 & 53.66 \\
\hline & 7.0 & 54.15 & 53.38 & 55.98 & 56.86 & 57.04 & 57.04 & 56.86 & 55.97 & 53.28 & 53.63 \\
\hline & 7.5 & 54.17 & 52.91 & 53.21 & 53.46 & 53.62 & 53.62 & 53.46 & 53.20 & 52.82 & 53.62 \\
\hline \multirow{9}{*}{ B-2 } & 7.5 & 54.17 & 52.91 & 53.21 & 53.46 & 53.62 & 53.62 & 53.46 & 53.20 & 52.82 & 53.62 \\
\hline & 8.0 & 54.35 & 53.53 & 56.28 & 57.21 & 57.40 & 57.40 & 57.21 & 56.27 & 53.43 & 53.78 \\
\hline & 9.0 & 54.82 & 56.04 & 87.90 & 91.34 & 91.55 & 91.55 & 91.34 & 87.90 & 56.01 & 54.72 \\
\hline & 10.0 & 55.03 & 56.28 & 88.71 & 92.23 & 92.46 & 92.46 & 92.23 & 88.71 & 56.21 & 54.79 \\
\hline & 11.0 & 55.28 & 56.55 & 89.60 & 93.20 & 93.46 & 93.46 & 93.20 & 89.60 & 56.44 & 54.78 \\
\hline & 12.0 & 55.49 & 56.77 & 90.14 & 93.79 & 94.07 & 94.07 & 93.79 & 90.14 & 56.65 & 54.81 \\
\hline & 13.0 & 55.70 & 56.97 & 90.39 & 94.07 & 94.37 & 94.37 & 94.07 & 90.38 & 56.86 & 55.07 \\
\hline & 14.0 & 55.88 & 57.09 & 90.91 & 94.60 & 94.91 & 94.91 & 94.60 & 90.90 & 56.98 & 55.19 \\
\hline & 15.0 & 55.78 & 54.31 & 54.93 & 55.45 & 55.78 & 55.78 & 55.45 & 54.92 & 54.18 & 55.06 \\
\hline \multirow{9}{*}{ B-3 } & 15.0 & 55.78 & 54.31 & 54.93 & 55.45 & 55.78 & 55.78 & 55.45 & 54.92 & 54.18 & 55.06 \\
\hline & 16.0 & 56.28 & 57.54 & 91.76 & 95.54 & 95.87 & 95.87 & 95.54 & 91.75 & 57.40 & 55.47 \\
\hline & 17.0 & 56.48 & 57.78 & 92.39 & 96.22 & 96.58 & 96.58 & 96.22 & 92.39 & 57.69 & 56.15 \\
\hline & 18.0 & 56.66 & 57.98 & 92.62 & 96.48 & 96.89 & 96.89 & 96.48 & 92.61 & 57.93 & 56.49 \\
\hline & 19.0 & 56.86 & 58.21 & 92.88 & 96.79 & 97.23 & 97.23 & 96.79 & 92.87 & 58.11 & 56.50 \\
\hline & 20.0 & 57.02 & 58.39 & 92.96 & 96.90 & 97.36 & 97.36 & 96.90 & 92.95 & 58.25 & 56.36 \\
\hline & 21.0 & 57.19 & 58.59 & 93.01 & 96.98 & 97.46 & 97.46 & 96.98 & 93.00 & 58.45 & 56.45 \\
\hline & 22.0 & 57.11 & 56.30 & 59.79 & 61.20 & 61.69 & 61.69 & 61.20 & 59.78 & 56.16 & 56.33 \\
\hline & 22.5 & 57.09 & 55.76 & 56.59 & 57.31 & 57.77 & 57.77 & 57.30 & 56.58 & 55.71 & 56.97 \\
\hline \multirow{9}{*}{ B-4 } & 22.5 & 57.09 & 55.76 & 56.59 & 57.31 & 57.77 & 57.77 & 57.30 & 56.58 & 55.71 & 56.97 \\
\hline & 23.0 & 57.24 & 56.44 & 59.91 & 61.33 & 61.82 & 61.81 & 61.33 & 59.90 & 56.30 & 56.45 \\
\hline & 24.0 & 57.62 & 59.12 & 93.88 & 97.97 & 98.47 & 98.46 & 97.96 & 93.87 & 58.96 & 56.73 \\
\hline & 25.0 & 57.75 & 59.30 & 93.90 & 98.02 & 98.54 & 98.54 & 98.02 & 93.89 & 59.15 & 57.06 \\
\hline & 26.0 & 57.88 & 59.54 & 94.24 & 98.44 & 98.98 & 98.98 & 98.43 & 94.23 & 59.44 & 57.55 \\
\hline & 27.0 & 58.00 & 59.72 & 94.27 & 98.48 & 99.05 & 99.05 & 98.48 & 94.26 & 59.67 & 57.80 \\
\hline & 28.0 & 58.10 & 59.92 & 94.46 & 98.72 & 99.30 & 99.30 & 98.71 & 94.44 & 59.81 & 57.73 \\
\hline & 29.0 & 58.15 & 60.02 & 94.66 & 98.91 & 99.50 & 99.49 & 98.91 & 94.65 & 59.87 & 57.48 \\
\hline & 30.0 & 57.95 & 57.26 & 58.35 & 59.24 & 59.82 & 59.82 & 59.23 & 58.33 & 57.10 & 57.15 \\
\hline \multirow{9}{*}{ B-5 } & 30.0 & 57.95 & 57.26 & 58.35 & 59.24 & 59.82 & 59.82 & 59.23 & 58.33 & 57.10 & 57.15 \\
\hline & 31.0 & 58.30 & 60.25 & 93.28 & 97.37 & 97.95 & 97.95 & 97.36 & 93.26 & 60.12 & 57.63 \\
\hline & 32.0 & 58.39 & 60.44 & 93.22 & 97.33 & 97.93 & 97.93 & 97.32 & 93.21 & 60.30 & 57.63 \\
\hline & 33.0 & 58.44 & 60.61 & 93.38 & 97.52 & 98.14 & 98.14 & 97.51 & 93.37 & 60.46 & 57.61 \\
\hline & 34.0 & 58.49 & 60.80 & 93.27 & 97.41 & 98.05 & 98.05 & 97.41 & 93.26 & 60.65 & 57.83 \\
\hline & 35.0 & 58.53 & 60.96 & 93.21 & 97.34 & 97.99 & 97.99 & 97.34 & 93.19 & 60.86 & 58.18 \\
\hline & 36.0 & 58.56 & 61.10 & 92.72 & 96.81 & 97.47 & 97.47 & 96.80 & 92.70 & 61.03 & 58.29 \\
\hline & 37.0 & 58.36 & 59.08 & 62.69 & 64.29 & 64.97 & 64.96 & 64.28 & 62.67 & 58.98 & 58.09 \\
\hline & 37.5 & 58.28 & 58.61 & 59.90 & 60.89 & 61.55 & 61.55 & 60.88 & 59.87 & 58.47 & 57.58 \\
\hline \multirow{9}{*}{ B-6 } & 37.5 & 58.28 & 58.61 & 59.90 & 60.89 & 61.55 & 61.55 & 60.88 & 59.88 & 58.47 & 57.58 \\
\hline & 38.0 & 58.35 & 59.16 & 62.66 & 64.22 & 64.88 & 64.88 & 64.21 & 62.63 & 59.02 & 57.76 \\
\hline & 39.0 & 58.54 & 61.44 & 92.38 & 96.39 & 97.05 & 97.05 & 96.38 & 92.36 & 61.31 & 57.89 \\
\hline & 40.0 & 58.52 & 61.47 & 90.99 & 94.85 & 95.52 & 95.51 & 94.84 & 90.97 & 61.35 & 57.88 \\
\hline & 41.0 & 58.49 & 61.50 & 89.42 & 93.09 & 93.77 & 93.77 & 93.09 & 89.40 & 61.38 & 57.92 \\
\hline & 42.0 & 58.45 & 61.52 & 87.93 & 91.44 & 92.13 & 92.12 & 91.43 & 87.91 & 61.40 & 57.81 \\
\hline & 43.0 & 58.41 & 61.60 & 87.84 & 91.32 & 92.01 & 92.00 & 91.31 & 87.83 & 61.49 & 57.91 \\
\hline & 44.0 & 58.31 & 61.54 & 86.10 & 89.37 & 90.04 & 90.03 & 89.36 & 86.08 & 61.46 & 58.07 \\
\hline & 45.0 & 58.08 & 59.66 & 61.08 & 62.11 & 62.80 & 62.80 & 62.10 & 61.06 & 59.62 & 57.95 \\
\hline
\end{tabular}


Table B - 32: Temperature $\left({ }^{\circ} \mathrm{C}\right)$ map of the west side of position B at EOC 145A (total 106.4 EFPD).

\begin{tabular}{|c|c|c|c|c|c|c|c|c|c|c|c|}
\hline \multirow{2}{*}{$\begin{array}{c}\text { Plate } \\
\text { Location }\end{array}$} & \multirow{2}{*}{$\begin{array}{c}\text { Axial } \\
\text { Location (in) }\end{array}$} & \multicolumn{10}{|c|}{ Width of Plate (inches) } \\
\hline & & 0.00 & 0.08 & 0.16 & 0.25 & 0.50 & 1.00 & 1.50 & 2.00 & 2.08 & 2.16 \\
\hline \multirow{9}{*}{ B-1 } & 0.0 & 51.67 & 51.79 & 51.76 & 51.77 & 51.77 & 51.77 & 51.77 & 51.76 & 51.79 & 51.67 \\
\hline & 1.0 & 53.29 & 53.88 & 75.39 & 77.75 & 77.82 & 77.82 & 77.75 & 75.39 & 53.86 & 53.17 \\
\hline & 2.0 & 53.55 & 54.21 & 78.26 & 80.86 & 80.95 & 80.95 & 80.86 & 78.26 & 54.17 & 53.11 \\
\hline & 3.0 & 53.79 & 54.40 & 78.70 & 81.37 & 81.47 & 81.47 & 81.37 & 78.70 & 54.35 & 53.07 \\
\hline & 4.0 & 54.08 & 54.68 & 80.76 & 83.59 & 83.71 & 83.71 & 83.59 & 80.75 & 54.65 & 53.39 \\
\hline & 5.0 & 54.34 & 54.98 & 82.85 & 85.86 & 85.99 & 85.99 & 85.86 & 82.85 & 54.93 & 53.50 \\
\hline & 6.0 & 54.63 & 55.33 & 85.24 & 88.44 & 88.58 & 88.58 & 88.44 & 85.24 & 55.27 & 53.70 \\
\hline & 7.0 & 54.68 & 53.23 & 55.81 & 56.65 & 56.80 & 56.80 & 56.65 & 55.80 & 53.17 & 53.75 \\
\hline & 7.5 & 54.73 & 52.76 & 53.02 & 53.24 & 53.36 & 53.36 & 53.23 & 53.02 & 52.69 & 53.77 \\
\hline \multirow{9}{*}{ B-2 } & 7.5 & 54.73 & 52.76 & 53.02 & 53.24 & 53.36 & 53.36 & 53.23 & 53.02 & 52.69 & 53.77 \\
\hline & 8.0 & 54.91 & 53.37 & 56.08 & 56.97 & 57.12 & 57.12 & 56.97 & 56.08 & 53.30 & 53.90 \\
\hline & 9.0 & 55.36 & 55.87 & 87.64 & 91.02 & 91.19 & 91.19 & 91.02 & 87.64 & 55.84 & 55.39 \\
\hline & 10.0 & 55.60 & 56.08 & 88.42 & 91.86 & 92.05 & 92.05 & 91.86 & 88.41 & 56.03 & 55.32 \\
\hline & 11.0 & 55.87 & 56.32 & 89.26 & 92.77 & 92.98 & 92.97 & 92.77 & 89.25 & 56.24 & 55.02 \\
\hline & 12.0 & 56.10 & 56.51 & 89.75 & 93.31 & 93.52 & 93.52 & 93.30 & 89.74 & 56.42 & 54.79 \\
\hline & 13.0 & 56.31 & 56.68 & 89.95 & 93.52 & 93.75 & 93.75 & 93.52 & 89.94 & 56.60 & 55.08 \\
\hline & 14.0 & 56.51 & 56.77 & 90.42 & 93.99 & 94.22 & 94.22 & 93.99 & 90.41 & 56.69 & 55.17 \\
\hline & 15.0 & 56.47 & 53.96 & 54.46 & 54.88 & 55.13 & 55.13 & 54.87 & 54.45 & 53.86 & 55.18 \\
\hline \multirow{9}{*}{ B-3 } & 15.0 & 56.47 & 53.96 & 54.46 & 54.88 & 55.13 & 55.13 & 54.87 & 54.45 & 53.86 & 55.18 \\
\hline & 16.0 & 56.95 & 57.17 & 91.20 & 94.86 & 95.10 & 95.09 & 94.85 & 91.19 & 57.06 & 55.54 \\
\hline & 17.0 & 57.14 & 57.39 & 91.80 & 95.50 & 95.76 & 95.76 & 95.50 & 91.79 & 57.32 & 56.77 \\
\hline & 18.0 & 57.33 & 57.57 & 91.99 & 95.72 & 95.99 & 95.99 & 95.71 & 91.98 & 57.52 & 57.30 \\
\hline & 19.0 & 57.53 & 57.77 & 92.20 & 95.95 & 96.24 & 96.24 & 95.94 & 92.19 & 57.69 & 57.10 \\
\hline & 20.0 & 57.69 & 57.92 & 92.24 & 95.99 & 96.30 & 96.30 & 95.99 & 92.23 & 57.81 & 56.60 \\
\hline & 21.0 & 57.86 & 58.09 & 92.25 & 96.00 & 96.32 & 96.32 & 96.00 & 92.23 & 57.98 & 56.51 \\
\hline & 22.0 & 57.81 & 55.77 & 59.09 & 60.36 & 60.73 & 60.73 & 60.35 & 59.07 & 55.66 & 56.29 \\
\hline & 22.5 & 57.80 & 55.21 & 55.88 & 56.44 & 56.79 & 56.78 & 56.43 & 55.86 & 55.16 & 57.92 \\
\hline \multirow{9}{*}{ B-4 } & 22.5 & 57.80 & 55.21 & 55.88 & 56.44 & 56.79 & 56.78 & 56.43 & 55.86 & 55.16 & 57.92 \\
\hline & 23.0 & 57.93 & 55.88 & 59.20 & 60.46 & 60.83 & 60.83 & 60.45 & 59.18 & 55.78 & 56.41 \\
\hline & 24.0 & 58.27 & 58.55 & 93.02 & 96.82 & 97.15 & 97.15 & 96.82 & 93.01 & 58.42 & 56.63 \\
\hline & 25.0 & 58.38 & 58.71 & 93.01 & 96.82 & 97.18 & 97.17 & 96.81 & 92.99 & 58.58 & 57.24 \\
\hline & 26.0 & 58.49 & 58.91 & 93.30 & 97.15 & 97.54 & 97.54 & 97.14 & 93.29 & 58.82 & 58.18 \\
\hline & 27.0 & 58.60 & 59.06 & 93.28 & 97.14 & 97.54 & 97.54 & 97.13 & 93.26 & 58.99 & 58.55 \\
\hline & 28.0 & 58.68 & 59.22 & 93.42 & 97.31 & 97.72 & 97.72 & 97.30 & 93.40 & 59.13 & 58.26 \\
\hline & 29.0 & 58.71 & 59.29 & 93.56 & 97.43 & 97.84 & 97.84 & 97.42 & 93.54 & 59.16 & 57.58 \\
\hline & 30.0 & 58.54 & 56.48 & 57.35 & 58.04 & 58.48 & 58.47 & 58.03 & 57.32 & 56.35 & 57.09 \\
\hline \multirow{9}{*}{ B-5 } & 30.0 & 58.54 & 56.48 & 57.35 & 58.04 & 58.48 & 58.48 & 58.03 & 57.32 & 56.35 & 57.09 \\
\hline & 31.0 & 58.82 & 59.47 & 92.11 & 95.82 & 96.21 & 96.20 & 95.81 & 92.08 & 59.35 & 57.57 \\
\hline & 32.0 & 58.87 & 59.62 & 92.01 & 95.72 & 96.14 & 96.13 & 95.71 & 91.99 & 59.50 & 57.43 \\
\hline & 33.0 & 58.90 & 59.77 & 92.13 & 95.85 & 96.28 & 96.28 & 95.83 & 92.10 & 59.63 & 57.35 \\
\hline & 34.0 & 58.91 & 59.91 & 91.96 & 95.67 & 96.12 & 96.12 & 95.66 & 91.93 & 59.78 & 57.81 \\
\hline & 35.0 & 58.92 & 60.04 & 91.83 & 95.54 & 95.99 & 95.99 & 95.52 & 91.81 & 59.94 & 58.51 \\
\hline & 36.0 & 58.90 & 60.14 & 91.29 & 94.94 & 95.38 & 95.38 & 94.92 & 91.26 & 60.05 & 58.64 \\
\hline & 37.0 & 58.71 & 58.07 & 61.42 & 62.80 & 63.30 & 63.30 & 62.78 & 61.38 & 57.97 & 58.49 \\
\hline & 37.5 & 58.62 & 57.59 & 58.60 & 59.36 & 59.85 & 59.85 & 59.35 & 58.56 & 57.46 & 57.37 \\
\hline \multirow{9}{*}{ B-6 } & 37.5 & 58.62 & 57.59 & 58.60 & 59.36 & 59.85 & 59.85 & 59.35 & 58.56 & 57.46 & 57.37 \\
\hline & 38.0 & 58.66 & 58.13 & 61.36 & 62.70 & 63.20 & 63.19 & 62.68 & 61.32 & 58.00 & 57.69 \\
\hline & 39.0 & 58.77 & 60.39 & 90.82 & 94.40 & 94.83 & 94.82 & 94.39 & 90.79 & 60.26 & 57.62 \\
\hline & $\begin{array}{l}40.0 \\
\end{array}$ & 58.72 & 60.39 & 89.39 & 92.85 & 93.27 & 93.27 & 92.83 & 89.35 & 60.27 & 57.50 \\
\hline & 41.0 & 58.65 & 60.38 & 87.75 & 91.07 & 91.51 & 91.50 & 91.06 & 87.72 & 60.26 & 57.60 \\
\hline & 42.0 & 58.56 & 60.37 & 86.20 & 89.40 & 89.84 & 89.83 & 89.38 & 86.17 & 60.24 & 57.39 \\
\hline & 43.0 & 58.48 & 60.42 & 86.07 & 89.22 & 89.66 & 89.66 & 89.21 & 86.03 & 60.30 & 57.67 \\
\hline & 44.0 & 58.34 & 60.32 & 84.26 & 87.24 & 87.67 & 87.66 & 87.22 & 84.22 & 60.23 & 58.03 \\
\hline & 45.0 & 58.11 & 58.42 & 59.53 & 60.32 & 60.82 & 60.82 & 60.29 & 59.49 & 58.35 & 57.99 \\
\hline
\end{tabular}


